\title{
Improving ventricular pacing in adults and children : to treat or to avoid dyssynchrony-induced cardiac failure
}

Citation for published version (APA):

van Geldorp, I. E. G. (2012). Improving ventricular pacing in adults and children : to treat or to avoid dyssynchrony-induced cardiac failure. [Doctoral Thesis, Maastricht University]. Maastricht University. https://doi.org/10.26481/dis.20121116ig

Document status and date:

Published: 01/01/2012

DOI:

10.26481/dis.20121116ig

Document Version:

Publisher's PDF, also known as Version of record

\section{Please check the document version of this publication:}

- A submitted manuscript is the version of the article upon submission and before peer-review. There can be important differences between the submitted version and the official published version of record.

People interested in the research are advised to contact the author for the final version of the publication, or visit the DOI to the publisher's website.

- The final author version and the galley proof are versions of the publication after peer review.

- The final published version features the final layout of the paper including the volume, issue and page numbers.

Link to publication

\footnotetext{
General rights rights.

- You may freely distribute the URL identifying the publication in the public portal. please follow below link for the End User Agreement:

www.umlib.nl/taverne-license

Take down policy

If you believe that this document breaches copyright please contact us at:

repository@maastrichtuniversity.nl

providing details and we will investigate your claim.
}

Copyright and moral rights for the publications made accessible in the public portal are retained by the authors and/or other copyright owners and it is a condition of accessing publications that users recognise and abide by the legal requirements associated with these

- Users may download and print one copy of any publication from the public portal for the purpose of private study or research.

- You may not further distribute the material or use it for any profit-making activity or commercial gain

If the publication is distributed under the terms of Article $25 \mathrm{fa}$ of the Dutch Copyright Act, indicated by the "Taverne" license above, 


\title{
IMPROVING VENTRICULAR PACING IN ADULTS AND CHILDREN
}

\author{
TO TREAT OR TO AVOID \\ DYSSYNCHRONY-INDUCED CARDIAC FAILURE
}

Irene van Geldorp 


\title{
IMPROVING VENTRICULAR PACING IN ADULTS AND CHILDREN
}

\author{
TO TREAT OR TO AVOID \\ DYSSYNCHRONY-INDUCED CARDIAC FAILURE
}

\begin{abstract}
PROEFSCHRIFT
ter verkrijging van de graad van doctor aan de Universiteit Maastricht, op gezag van de Rector Magnificus, prof.dr. L.L.G. Soete, volgens het besluit van het College van Decanen in het openbaar te verdedigen op vrijdag 16 november 2012 om 10.00 uur

door

Irene Elisabeth Gerardine van Geldorp
\end{abstract}

Geboren op 6 april 1981

te Utrecht, Nederland 


\section{Promotores:}

Prof.dr. T. Delhaas

Prof.dr. F.W. Prinzen

Prof.dr. J. Janoušek (University Hospital Motol, Prague, Czech Republic)

\section{Beoordelingscommissie:}

Prof.dr. U. Schotten (voorzitter)

Prof.dr.ir. T. Arts

Prof.dr. N. Blom (Universiteit Leiden)

Prof.dr. H.P. Brunner-La Rocca

Prof.dr. L.J.I. Zimmermann

Part of the research described in this thesis was supported by a grant of the Dutch Heart Foundation (DHF-2010T078)

Financial support by the Dutch Heart Foundation and Stichting Hartsvrienden RESCAR for the publication of this thesis is gratefully acknowledged 
voor alle kinderen en (jong)volwassenen die de pech hebben dat hun hart kunstmatig gestimuleerd moet worden, alsook voor hun familie en vrienden

met Reinder in gedachten

(1981-2012) 
Colofon

ISBN/EAN:

978-90-8590-054-2

Cover:

Ontwerp: Irene van Geldorp

Lay-out:

Eric Lemmens - D\&L graphics

Published by:

www.dlgraphics.nl

Printed by:

Schrijen-Lippertz 


\section{TABLE OF CONTENTS}

\section{Chapter 1}

General Introduction

Background and General Aim of the Thesis

\section{Chapter 2}

Background of the Thesis

Consequences of Abnormal Ventricular Electrical Activation

\section{Chapter 3}

Beneficial Effects of Biventricular Pacing in Chronically Right Ventricular Paced Patients with Mild Cardiomyopathy

\section{Chapter 4}

Comparison of a Non-Invasive Arterial Pulse Contour Technique and Echo Doppler Aorta Velocity-Time Integral on Stroke Volume Changes in Optimization of Cardiac Resynchronization Therapy

\section{Chapter 5}

Chronic Left Ventricular Pacing Preserves Left Ventricular Function in Children

\section{Chapter 6}

Impact of the Permanent Ventricular Pacing Site on Left Ventricular Function in Children: a Retrospective Multicentre Survey

\section{Chapter 7}

Permanent Cardiac Pacing in Children - Choosing the Optimal Pacing-site:

A Multi-Center Study

\section{Chapter 8}

Acute Hemodynamic Benefits of Biventricular and Single-Site Systemic Ventricular Pacing in Patients with a Systemic Right Ventricle

\section{Chapter 9}

Pacing Strategies Toward Better Treatment of Dyssynchrony and Prevention of PacingInduced Cardiac Deterioration 



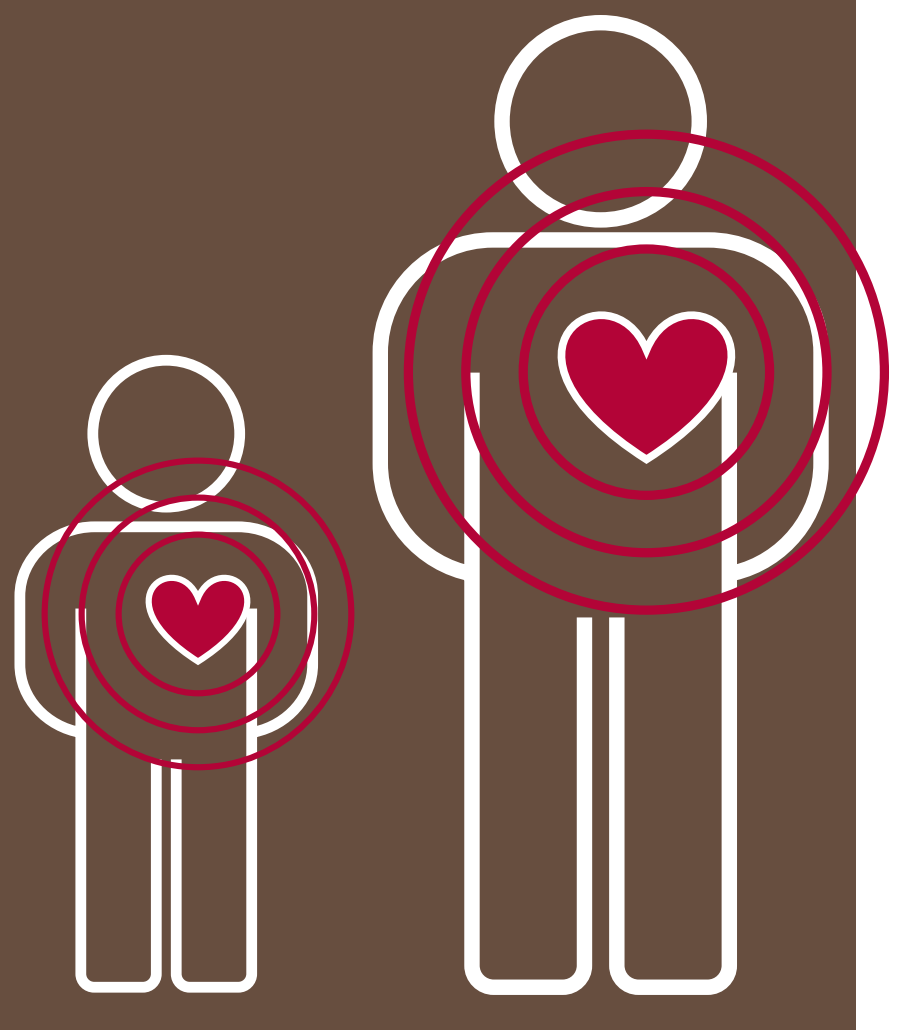

ש

$\stackrel{0}{+}$

4

E

-

e

0 을

e 0

드

10 0

(1) 은

E $v$

(1) 10

( 
Chapter 1 


\section{INTRODUCTION}

Synchronous, coordinate contraction of the ventricles, as established by normal conduction in healthy hearts, is important for good cardiac pump function. Under physiological circumstances, the electrical impulse originating from the sinus node in the right atrium passes the atrioventricular (AV) node, and is then rapidly conducted via the specialized His-Purkinje conduction system to both ventricles simultaneously. This results in almost synchronous electrical activation and, consequently, in a coordinate contraction of the myocytes.

\section{Dyssynchrony may deteriorate cardiac function}

In hearts with a partially disrupted conduction system, as well as in ventricularly paced hearts, the physiological pattern of electrical ventricular activation is adversely affected. The delayed left ventricular (LV) electrical activation in left bundle branch block (LBBB) is accompanied by abnormal dyssynchronous mechanical interactions within the LV. ${ }^{1,2}$ The sequence of activation in conventional, right ventricular (RV), pacing resembles the pattern as in LBBB. ${ }^{3}$ Both, intrinsic and pacing-induced LBBB, are associated with impairment of LV function, structural remodelling of the $L V$, and an increased risk for heart failure..$^{4-9}$ Efforts should be made to better treat, as well as to prevent, cardiac deterioration originating from either intrinsic or pacing-induced dyssynchrony.

\section{Cardiac resynchronization therapy}

Cardiac resynchronization therapy (CRT) aims to reverse the deleterious effects that may originate from LV dyssynchrony, by restoration of a more coordinated contraction pattern by means of biventricular (BiV) pacing. Biventricular pacing improves clinical presentation, reduces mortality, reverses LV remodeling and improves LV function in the majority of patients with severe LV dysfunction and severe clinical heart failure associated with either $\mathrm{LBBB},{ }^{10-15}$ or RV pacing-induced dyssynchrony. ${ }^{16,17}$ Consequently, CRT is nowadays incorporated in the management of severe LV dysfunction and clinical heart failure associated with dyssynchrony. However, the individual response to CRT varies widely between patients, with inadequate response in up to $30-50 \%$ of the patients receiving CRT. The proportion of these 'non-responders' may be diminished by individual optimization of CRT-delivery and better pre-implant selection of CRT-candidates. At the other hand, also patients with less severe heart failure might benefit from CRT, but they do currently not fulfill the criteria to receive this therapy. Conceivably the question may arise whether the indication criteria for CRT should be extended. In deduction, several aspects of CRT should be optimized to increase the overall success rate and the individual benefits of CRT. 


\section{Chronic ventricular pacing for complete atrioventricular block}

In patients with congenital or acquired complete atrioventricular (AV) block, ventricular pacing is indicated to increase heart rate. An important side effect of ventricular pacing is the induction of abnormal electrical activation patterns, which may cause mechanical dyssynchrony. In contrast to adults with intrinsic LBBB, the objective in pediatric patients with isolated AV block should be to prevent pacing-induced dyssynchrony, rather than to treat dyssynchrony. Conventionally, ventricular pacemaker leads are positioned in or at the RV. Chronic RV pacing is associated with an acute and chronic impairment of LV function, structural remodeling of the LV, and increased risk of heart failure. ${ }^{8,18-22}$ Overt heart failure is reported in about 7\%, and impaired LV function in up to $13 \%$ of the chronically RV-paced pediatric patients after follow-up for about one decade. $^{19,23-25}$ The actual incidence of ventricular dysfunction in relation to chronic RV pacing may in fact be higher for life-long follow-up. Therefore, in children requiring chronic ventricular pacing, the prevention of pacing-induced functional and structural deterioration should be a major aim.

\section{Aim OF THE Thesis}

The general aim of the research presented in this thesis is to improve pacing therapy in adults and children to better treat electromechanical ventricular dyssynchrony and to avoid pacinginduced cardiac deterioration.

\section{Toward optimization of CRT to better treat dyssynchrony}

To improve treatment of dyssynchrony, optimization of CRT should be achieved in several

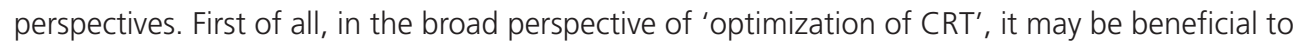
refine indication-criteria for CRT. We sought therefore to investigate the effect of BiV pacing in patients who may benefit from (re)synchronization of electrical activation, but who do not meet current indication criteria for CRT. Secondly, benefits induced by CRT may be improved by tailoring of device-settings to the individual patient. ${ }^{26-29}$ However, procedures to optimize device-settings, as well as measurements to guide these optimization procedures, are still under debate. We sought for a patient-friendly non-invasive measurement to guide optimization procedures.

\section{Toward prevention of pacing-induced deterioration: 'optimal-site pacing'}

Especially in children the preservation of cardiac function during chronic ventricular pacing should take high priority, because they have a perspective of life-long pacing. The awareness of the potentially deleterious effects of conventional pacing has initiated the search for alternative approaches for chronic ventricular pacing. The degree of pacing-induced dyssynchrony seems to be a major determinant of cardiac pump function and appears to vary between the different 
pacing sites. ${ }^{30-34}$ Aiming at the prevention of pacing-associated functional and structural deterioration, we sought therefore to define strategies for 'optimal' chronic ventricular pacing in the young.

\section{Outline of the Thesis}

The general background and introduction to the studies presented in this thesis are given in chapter 2. Herein, the importance of the sequence of activation and the pathophysiology of ventricular pacing are elucidated.

Pertaining to the aim to improve treatment of dyssynchrony, our research on the optimization of CRT in adult patients is presented in chapters 3 and 4 . In the perspective that CRT may also be beneficial in patients with mild forms of dyssynchrony-associated LV dysfunction, we investigated the effects of permanent BiV pacing and RV pacing in a cross-over study in previously chronically RV paced patients with mild cardiomyopathy (chapter 3 ). With respect to optimization of CRT, great clinical need exits for a harmless, patient friendly and easy-to-use technique suitable for being applied in individual optimization procedures. We investigated whether Nexfin CO-Trek ${ }^{\circledR}$ (BMEYE B.V., Amsterdam, Netherlands), a non-invasive method to continuously monitor hemodynamics using a finger sensor, reliably measures beat-to-beat changes in stroke volume, as well as immediate effects of CRT at various AV-delays and, hence, whether its use is feasible in the individual optimization of CRT (chapter 4).

The studies with the objective to define better pacing-strategies aiming at prevention of pacinginduced cardiac deterioration in children are presented in chapters 5-8. Given the acute beneficial hemodynamic effects of single-site LV pacing shown by earlier studies of our group, ${ }^{32,}$ 34, 35 we investigated whether chronic LV pacing is superior to RV pacing in children with structurally normal hearts. A small pilot study is presented in which we surveyed LV function in children with isolated AV block, undergoing chronic epicardial pacing at either the RV or LV (chapter 5). Subsequently, influences of chronic ventricular pacing in children with isolated AV block were retrospectively, as well as cross-sectionally, evaluated in a large observational multicenter study (chapters 6 and 7). In patients with transposition of the great arteries treated by atrial redirection surgery, we investigated the acute hemodynamic effects of conventional nonsystemic ventricular pacing, systemic ventricular pacing, and biventricular pacing (chapter 8).

In the last chapter of this thesis (chapter 9), the findings of above-mentioned studies are linked and put in broader perspective. The first part of the general discussion concerns optimization of CRT. In the second part, several sites for chronic ventricular pacing are reviewed and clinical implications of these sites are discussed. 
Chapter 1 


\section{REFERENCES}

1. Witte KK, Pipes RR, Nanthakumar K, Parker JD. Biventricular pacemaker upgrade in previously paced heart failure patients-improvements in ventricular dyssynchrony. J Card Fail 2006; 12(3):199-204.

2. Little WC, Reeves RC, Arciniegas J, Katholi RE, Rogers EW. Mechanism of abnormal interventricular septal motion during delayed left ventricular activation. Circulation 1982; 65(7):1486-1491.

3. Vassallo JA, Cassidy DM, Miller JM, Buxton AE, Marchlinski FE, Josephson ME. Left ventricular endocardial activation during right ventricular pacing: effect of underlying heart disease. J Am Coll Cardiol 1986; 7(6):1228-1233.

4. Manolis AS. The deleterious consequences of right ventricular apical pacing: time to seek alternate site pacing. Pacing Clin Electrophysiol 2006; 29(3):298-315.

5. Moak JP, Hasbani K, Ramwell C, Freedenberg V, Berger JT, DiRusso G, Callahan P. Dilated cardiomyopathy following right ventricular pacing for AV block in young patients: resolution after upgrading to biventricular pacing systems. J Cardiovasc Electrophysiol 2006; 17(10):1068-1071.

6. Sweeney MO, Hellkamp AS, Ellenbogen KA, Greenspon AJ, Freedman RA, Lee KL, Lamas GA. Adverse effect of ventricular pacing on heart failure and atrial fibrillation among patients with normal baseline QRS duration in a clinical trial of pacemaker therapy for sinus node dysfunction. Circulation 2003; 107(23):2932-2937.

7. Sweeney MO, Prinzen FW. A new paradigm for physiologic ventricular pacing. J Am Coll Cardiol 2006; 47(2):282-288.

8. Thambo JB, Bordachar P, Garrigue S, Lafitte S, Sanders P, Reuter S, Girardot R, Crepin D, Reant P, Roudaut R, Jais $P$, Haissaguerre M, Clementy J, Jimenez M. Detrimental ventricular remodeling in patients with congenital complete heart block and chronic right ventricular apical pacing. Circulation 2004; 110(25):3766-3772.

9. Vernooy K, Dijkman B, Cheriex EC, Prinzen FW, Crijns HJ. Ventricular remodeling during longterm right ventricular pacing following His bundle ablation. Am J Cardiol 2006; 97(8):1223-1227.

10. Abraham WT, Fisher WG, Smith AL, Delurgio DB, Leon AR, Loh E, Kocovic DZ, Packer M, Clavell AL, Hayes DL, Ellestad M, Trupp RJ, Underwood J, Pickering F, Truex C, McAtee P, Messenger J, the MSG. Cardiac Resynchronization in Chronic Heart Failure. N Engl J Med 2002; 346(24):18451853.

11. Cazeau S, Leclercq C, Lavergne T, Walker S, Varma C, Linde C, Garrigue S, Kappenberger L, Haywood GA, Santini M, Bailleul C, Daubert JC. Effects of multisite biventricular pacing in patients with heart failure and intraventricular conduction delay. N Engl J Med 2001; 344(12):873-880.

12. Cleland JG, Daubert JC, Erdmann E, Freemantle N, Gras D, Kappenberger L, Tavazzi L. The effect of cardiac resynchronization on morbidity and mortality in heart failure. N Engl J Med 2005; 352(15):1539-1549. 
13. Daubert JC, Leclercq C, Donal E, Mabo P. Cardiac resynchronisation therapy in heart failure: current status. Heart Fail Rev 2006; 11(2):147-154.

14. Donal E, Leclercq C, Linde C, Daubert JC. Effects of cardiac resynchronization therapy on disease progression in chronic heart failure. Eur Heart J 2006; 27(9):1018-1025.

15. St John Sutton MG, Plappert T, Abraham WT, Smith AL, DeLurgio DB, Leon AR, Loh E, Kocovic DZ, Fisher WG, Ellestad M, Messenger J, Kruger K, Hilpisch KE, Hill MR. Effect of cardiac resynchronization therapy on left ventricular size and function in chronic heart failure. Circulation 2003; 107(15):1985-1990.

16. Leclercq C, Cazeau S, Lellouche D, Fossati F, Anselme F, Davy JM, Sadoul N, Klug D, Mollo L, Daubert JC. Upgrading from single chamber right ventricular to biventricular pacing in permanently paced patients with worsening heart failure: The RD-CHF Study. Pacing Clin Electrophysiol 2007; 30 Suppl 1:S23-30.

17. Laurenzi F, Achilli A, Avella A, Peraldo C, Orazi S, Perego GB, Cesario A, Valsecchi S, De Santo T, Puglisi $A$, Tondo $C$. Biventricular upgrading in patients with conventional pacing system and congestive heart failure: results and response predictors. Pacing Clin Electrophysiol 2007; 30(9):1096-1104.

18. Karpawich PP, Mital S. Comparative left ventricular function following atrial, septal, and apical single chamber heart pacing in the young. Pacing Clin Electrophysio/ 1997; 20(8 Pt 1):1983-1988.

19. Moak JP, Barron KS, Hougen TJ, Wiles HB, Balaji S, Sreeram N, Cohen MH, Nordenberg A, Van Hare GF, Friedman RA, Perez M, Cecchin F, Schneider DS, Nehgme RA, Buyon JP. Congenital heart block: development of late-onset cardiomyopathy, a previously underappreciated sequela. J Am Coll Cardiol 2001; 37(1):238-242.

20. Tantengco MV, Thomas RL, Karpawich PP. Left ventricular dysfunction after long-term right ventricular apical pacing in the young. J Am Coll Cardiol 2001; 37(8):2093-2100.

21. Karpawich PP. Chronic right ventricular pacing and cardiac performance: the pediatric perspective. Pacing Clin Electrophysiol 2004; 27(6 Pt 2):844-849.

22. Janousek J, Tomek V, Chaloupecky V, Gebauer RA. Dilated cardiomyopathy associated with dualchamber pacing in infants: improvement through either left ventricular cardiac resynchronization or programming the pacemaker off allowing intrinsic normal conduction. I Cardiovasc Electrophysiol 2004; 15(4):470-474.

23. Kim JJ, Friedman RA, Eidem BW, Cannon BC, Arora G, Smith EO, Fenrich AL, Kertesz NJ. Ventricular function and long-term pacing in children with congenital complete atrioventricular block. J Cardiovasc Electrophysiol 2007; 18(4):373-377.

24. Vatasescu R, Shalganov T, Paprika D, Kornyei L, Prodan Z, Bodor G, Szatmari A, Szili-Torok T. Evolution of left ventricular function in paediatric patients with permanent right ventricular pacing for isolated congenital heart block: a medium term follow-up. Europace 2007; 9(4):228-232. 
25. Gebauer RA, Tomek V, Salameh A, Marek J, Chaloupecky V, Gebauer R, Matejka T, Vojtovic P, Janousek J. Predictors of left ventricular remodelling and failure in right ventricular pacing in the young. Eur Heart J 2009; 30(9):1097-1104.

26. Auricchio A, Stellbrink C, Block M, Sack S, Vogt J, Bakker P, Klein H, Kramer A, Ding J, Salo R, Tockman B, Pochet T, Spinelli J. Effect of pacing chamber and atrioventricular delay on acute systolic function of paced patients with congestive heart failure. The Pacing Therapies for Congestive Heart Failure Study Group. The Guidant Congestive Heart Failure Research Group. Circulation 1999; 99(23):2993-3001.

27. Auricchio A, Stellbrink C, Sack S, Block M, Vogt J, Bakker P, Huth C, Schondube F, Wolfhard U, Bocker D, Krahnefeld O, Kirkels $\mathrm{H}$. Long-term clinical effect of hemodynamically optimized cardiac resynchronization therapy in patients with heart failure and ventricular conduction delay. J Am Coll Cardiol 2002; 39(12):2026-2033.

28. Perego GB, Chianca R, Facchini M, Frattola A, Balla E, Zucchi S, Cavaglia S, Vicini I, Negretto M, Osculati $\mathrm{G}$. Simultaneous vs. sequential biventricular pacing in dilated cardiomyopathy: an acute hemodynamic study. Eur J Heart Fail 2003; 5(3):305-313.

29. Whinnett ZI, Davies JE, Willson K, Manisty CH, Chow AW, Foale RA, Davies DW, Hughes AD, Mayet J, Francis DP. Haemodynamic effects of changes in atrioventricular and interventricular delay in cardiac resynchronisation therapy show a consistent pattern: analysis of shape, magnitude and relative importance of atrioventricular and interventricular delay. Heart 2006; 92(11):16281634.

30. Prinzen FW, Peschar M. Relation between the pacing induced sequence of activation and left ventricular pump function in animals. Pacing Clin Electrophysiol 2002; 25(4 Pt 1):484-498.

31. Wyman BT, Hunter WC, Prinzen FW, Faris OP, McVeigh ER. Effects of single- and biventricular pacing on temporal and spatial dynamics of ventricular contraction. Am J Physiol Heart Circ Physiol 2002; 282(1):H372-379.

32. Peschar M, de Swart $H$, Michels KJ, Reneman RS, Prinzen FW. Left ventricular septal and apex pacing for optimal pump function in canine hearts. J Am Coll Cardiol 2003; 41(7):1218-1226.

33. Puggioni E, Brignole M, Gammage M, Soldati E, Bongiorni MG, Simantirakis EN, Vardas P, Gadler F, Bergfeldt L, Tomasi C, Musso G, Gasparini G, Del Rosso A. Acute comparative effect of right and left ventricular pacing in patients with permanent atrial fibrillation. J Am Coll Cardiol 2004; 43(2):234-238.

34. Vanagt WY, Verbeek XA, Delhaas T, Mertens L, Daenen WJ, Prinzen FW. The left ventricular apex is the optimal site for pediatric pacing: correlation with animal experience. Pacing Clin Electrophysiol 2004; 27(6 Pt 2):837-843.

35. Vanagt WY, Verbeek XA, Delhaas T, Gewillig M, Mertens L, Wouters P, Meyns B, Daenen WJ, Prinzen FW. Acute hemodynamic benefit of left ventricular apex pacing in children. Ann Thorac Surg 2005; 79(3):932-936. 


Chapter 2 


\section{CONSEQUENCES OF ABNORMAL VENTRICULAR ELECTRICAL ACTIVATION}

\section{Normal electrical activation of the ventricles}

In the normal heart coordinated mechanical contraction of the ventricular myocytes enables pump function to circulate the blood through the lungs and body. Coordinate mechanical contraction is induced by synchronous electrical activation of the myocardium via the specialized cardiac conduction system. Under physiological circumstances, the electrical impulse originating from the sinus node in the right atrium passes the atrioventricular (AV) node, and is then rapidly conducted via the specialized His-Purkinje conduction system to both ventricles simultaneously. This results in almost synchronous electrical activation and, consequently, in a coordinate contraction of the myocytes. During normal activation, synchrony is observed between the ventricles (interventricular synchrony) and within each ventricle (intraventricular synchrony).* Left-intraventricular electrical synchrony during normal activation is illustrated by the left upper panel of Figure 1. Intraventricular and interventricular synchrony may be disturbed by pathological alterations in the sequence of electrical activation. These alterations may be induced either intrinsically by ventricular pre-excitation or disruptions of the conduction system, or artificially by ventricular stimulation (i.e., ventricular pacing).

* Literally, "synchronous activation" denominates simultaneous activation of all ventricular myocytes, which is not achieved under physiological circumstances. Despite the rapid propagation (3-4 m/s) of the electrical impulse through the conduction tissue, activation of the ventricular myocardium occurs over a certain amount of time. Normally, electrical activation starts at the endocardium of the apex and progresses toward the epicardium, as well as upwards to the base, resulting in a coordinated and energetically efficient mechanical contraction, which is crucial for optimal left ventricular performance. Therefore, the term "euchrony" would more correctly describe the normal timing and sequence of ventricular activation under physiological conditions. However, "electrical synchrony" and "synchronous activation" are generally used in the literature to denominate the physiological timing and sequence of electrical activation and are accordingly used throughout this thesis. 


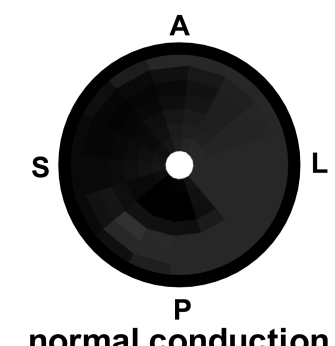

normal conduction

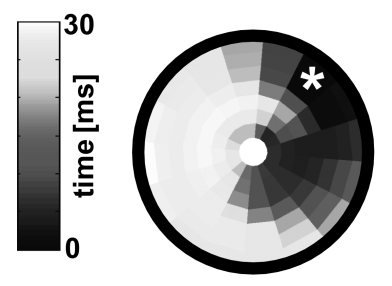

LV free wall pacing

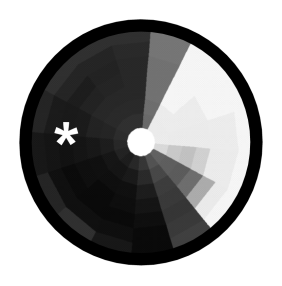

RV apical pacing

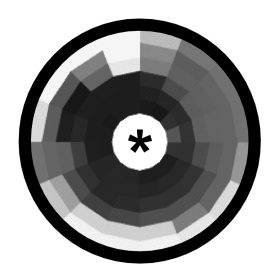

LV apical pacing

Figure 1

\section{Left ventricular endocardial activation in canine hearts}

Electrical activation mapping using a left ventricular (LV) intraventricular mapping catheter in canine experiments (described by Verbeek et al. JACC 2003),56 during normal activation, and while pacing from the right ventricular (RV) apex, LV free wall, and LV apex, respectively. The electrical activation maps are presented as bull's eye plots with the inner disk representing the LV apex and the outer disk representing the LV base. The letters A, P, S, and L indicate the anterior, posterior, septal, and lateral wall, respectively. Electrical activation of the LV is fast and synchronous during normal activation. During ventricular pacing, the region in the proximity of the pacing-site is early-activated, whereas myocardium remote from the pacing site is late-activated. In LV apical pacing, electrical activation is circumferentially synchronous. * $=$ ventricular pacing-site.

\section{Asynchronous electrical activation}

In hearts with ventricular pacing for complete AV block, as well as in hearts with a partially disrupted conduction system (e.g., left bundle branch block, LBBB), the initiation and the sequence of electrical ventricular activation are different from those in normal physiology. In the above-mentioned pathological circumstances, electrical activation of one or both ventricle(s) occurs exclusively through the slowly transmitting myocardium, instead of also through the rapidly conducting specialized conduction system. Slow cell-to-cell transmission of the electrical impulse results in asynchrony of electrical ventricular activation, with early activation of the myocytes close to the site where activation starts and delayed activation of the cells in remote regions (Figure 1, right upper panel). Consequently, early systolic shortening of the early- 
activated myocytes results in stretch of late-activated myocytes, rather than that it results in the onset of the ejection phase. When myocytes in remote regions are subsequently activated and start to contract, they contract even more powerfully due to the early systolic pre-stretching (known as the local Frank-Starling mechanism). ${ }^{1,2}$ Hence, electrical asynchrony, induced either by intrinsic conduction delays or by ventricular pacing, results in a 'dyssynchronous' contraction pattern, which is associated with a reduction in left ventricular (LV) pump function and an asymmetric redistribution of mechanical workload in the myocardium. The latter results in a redistribution of oxygen demand and perfusion, as well as in asymmetrical hypertrophy. ${ }^{1,3-6}$ However, the extent and importance of mechanical dyssynchrony are dependent on the pattern of electrical activation. 2,7

\section{Right ventricular pacing, as well as intrinsic left bundle branch block, results in ventricular dyssynchrony and is associated with cardiac deterioration}

Traditionally, ventricular pacemaker leads are positioned at the right ventricle (RV), since the RV apex and free wall are easily accessible, either at the epicardium via a surgical implantation or at the endocardium by means of a transvenous approach. These RV pacing sites are readily identified and associated with stable lead position and reliable capture. In hearts with normal left-right anatomy, RV pacing results in an LBBB-pattern of activation. ${ }^{2,8,9}$ In both intrinsic and RV pacing-induced LBBB-pattern the activation pattern is characterized by early activation of the RV and septum and delayed activation of the LV lateral wall, implicating electrical and mechanical asynchrony between the ventricles (interventricular asynchrony), as well as within each ventricle (intraventricular asynchrony). When the RV free wall starts to contract before the interventricular septum and the LV free wall, RV pressure increases before LV pressure is built up. As a consequence of this abnormal early systolic pressure gradient over the interventricular septum, the septum bulges into the LV, which can be observed as paradoxical movement of the septum. ${ }^{10}$ Mechanical dyssynchrony resulting from either intrinsic or pacing-induced LBBB, is associated with acute and chronic impairment of LV function, structural remodeling of the LV, and increased risk of heart failure. ${ }^{2,8,11-17}$ Additionally, the adverse relation between LBBBdyssynchrony and cardiac function is illustrated by the findings that morbidity and mortality are higher in chronic RV pacing when compared with atrial pacing only, ${ }^{18-21}$ and that prognosis is worse for heart failure patients with LBBB than for those without. ${ }^{22}$ Above-mentioned observations underline the importance of the efforts that should be made toward treatment and prevention of dyssynchrony originating from either intrinsic or pacing-induced LBBB.

\section{Toward treatment of dyssynchrony: cardiac resynchronization therapy}

Like normal patterns, adverse ventricular activation patterns can be manipulated by artificial ventricular stimulation. Ventricular pacing in order to induce a more synchronous pattern of activation in patients with dyssynchrony, is regularly referred to as 'cardiac resynchronization 
therapy' (CRT). By restoring a more coordinated contraction pattern by artificial stimulation prior to activation by means of abnormal intrinsic conduction, CRT aims to prevent adverse dyssynchronous contraction patterns. Typically, CRT is achieved by means of biventricular (BiV) pacing. The concept of electrical resynchronization is schematically represented in Figure 2. The first full paper reporting clinical benefit of CRT was published by Cazeau et al. in 1994. ${ }^{23}$ Subsequently, beneficial effects of BiV pacing in severe heart failure patients with wide QRS complex have consistently been reported by several clinical studies. ${ }^{24-26}$ Shortly after BiV pacing has been approved for clinical application (United States Food and Drug Administration in 2001), CRT has been recommended as an additional therapy for the treatment of patients with heart failure in the 2002 update of the American College of Cardiology/American Heart Association/North American Society of Pacing and Electrophysiology (ACC/AHA/NASPE). ${ }^{27}$

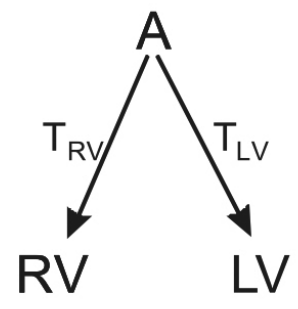

Normal
conduction

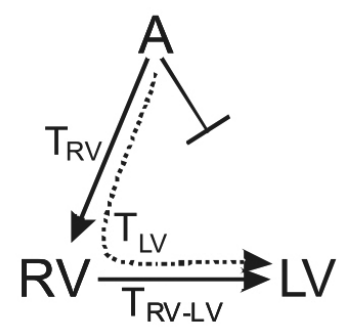

LBBB
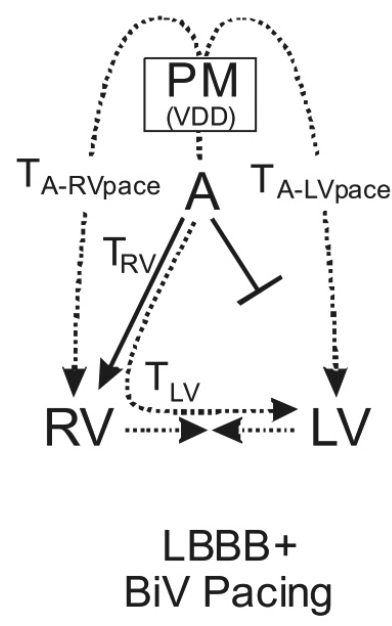

\section{Figure 2}

\section{Schematic pathway of ventricular activation fronts}

Schematic pathway of different ventricular activation fronts during normal conduction, left bundle branch block (LBBB) and LBBB with biventricular pacing. During normal conduction (left), activation of right ventricle (RV) and left ventricle (LV) occurs through intrinsic activation, and the time of activation ( $T_{R V}$ and $T_{\mathrm{LV}}$ ) is similar. During LBBB (middle), activation to the LV lateral wall ( $T_{\mathrm{LV}}$ ) is delayed because it occurs only by slow myocardial conduction ( $T_{\mathrm{RV}}-\mathrm{LV}$ ). During biventricular (BiV) pacing (right) RV and LV lateral wall can

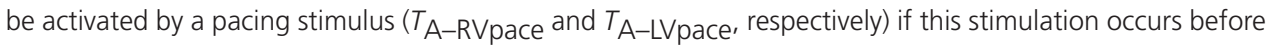
intrinsic activation. (After Vernooy et al. Heart Rhythm. 2007 Jan;4(1):75-82., with permission). ${ }^{57}$ 
Nowadays, CRT constitutes an established way of treatment for patients suffering from severe heart failure associated with conduction disturbances. Biventricular pacing improves LV function and reverses LV remodeling, as well as it improves clinical presentation and reduces mortality ${ }^{28}$ in the majority of patients with severe LV dysfunction and severe clinical heart failure associated with either LBBB25, 26, 29-32 or RV pacing-induced dyssynchrony. ${ }^{33-35}$ Therefore, BiV pacing is strongly recommended in patients with severe LV dysfunction (LV ejection fraction (LVEF) <35\%) and moderate to severe clinical heart failure (NYHA classification III-IV) associated with ventricular dyssynchrony (QRS >120ms). ${ }^{36}$ Based on the basic principle of CRT to prevent the heart from adverse dyssynchronous contraction patterns, CRT may also be beneficial in patients with milder forms of dyssynchrony-associated LV dysfunction (chapter 3).

Although collective benefit of CRT has been proven, appropriate individual response to CRT in terms of increase in LVEF and substantial reduction of LV dilatation appears to be absent in up to $30-50 \%$ of patients in whom CRT is applied. ${ }^{37}$ Individual response to CRT, and thus indirectly the overall success rate of CRT, could be improved by optimization of device settings. Ever since the first applications, devices for CRT have become significantly more sophisticated and include nowadays the capability to independently program atrioventricular (AV) and interventricular (ventriculo-ventricular; V) stimulation timing intervals. It has been shown that alterations in AV- or WV intervals acutely and significantly influence cardiac function. ${ }^{38-41}$ Although tailoring the AV-delay and W-interval to the individual patient may increase the haemodynamic improvement and long-term benefits brought by CRT, the best way to guide, as well as to perform, optimization of these device settings is still under debate. Besides the need for an evidence-based protocol for optimization, there is great clinical need for a harmless, patient friendly and easy-to-use technique, suitable to guide individual optimization of CRT (chapter 4).

\section{Toward prevention of pacing-induced dyssynchrony: optimal-site pacing}

In patients with complete AV block and bradycardia, ventricular pacing is indicated to increase heart rate, rather than to 'resynchronize' electrical activation. In addition to the obvious beneficial effects of the restoration of heart rate, chronic ventricular pacing cures the risk of sudden cardiac death and LV failure associated with untreated complete heart block. ${ }^{42,43}$ Therefore, AV block associated with symptomatic bradycardia is a class I indication for ventricular pacing therapy. ${ }^{44}$ Conventional RV pacing, however, induces an abnormal electrical activation pattern which causes mechanical dyssynchrony and is associated with impairment of pump function, LV remodeling, and increased risk of heart failure. 2, 8, 13, 14, 16, 17, 45 Right ventricular pacing, rather than etiology of AV block, has even been identified as an independent risk factor for the development of LV dilatation and dysfunction following chronic pacing. ${ }^{46,47}$ 
Prevention of mechanical dyssynchrony, and thus prevention of functional as well as structural deterioration, seems a new major challenge in patients that require chronic ventricular pacing. Above and beyond total activation time, the pattern of electrical activation is described by the sequence of activation in apex-base, left-right and endocard-epicard directions. This activationpattern seems to be a major determinant for cardiac pump function. ${ }^{2,48}$ During LV pacing in structural normal hearts, the LV lateral wall is activated prior to the septum and RV lateral wall, preventing the septum from paradoxical movements and resulting in superior hemodynamic performance as compared to RV pacing. ${ }^{10}$ On top of that, a physiological apex-to-base sequence of activation is induced by LV apical pacing, which results in synchronous electrical activation and contraction at the circumferential level of the LV (Figure 1, lower panels). ${ }^{49,50}$ In the acute setting of animal experiments and explorations in children undergoing cardiac surgery, LV apical pacing increases pump function when compared with RV pacing. ${ }^{51}$ In adult patients, single-site LV pacing has been shown to induce improvement in LV function comparable to the improvement by BiV pacing. ${ }^{52-54}$ Moreover, in a young child with severe LV failure following chronic RV pacing, impressive improvement of LV function and diameter was observed during chronic LV pacing. ${ }^{55}$ In deduction, the LV seems to provide promising alternative sites for chronic ventricular pacing (chapter 5-7). 


\section{REFERENCES}

1. Delhaas T, Arts T, Prinzen FW, Reneman RS. Regional fibre stress-fibre strain area as an estimate of regional blood flow and oxygen demand in the canine heart. J Physio/ 1994; 477 ( Pt 3):481496.

2. Prinzen FW, Peschar M. Relation between the pacing induced sequence of activation and left ventricular pump function in animals. Pacing Clin Electrophysio/ 2002; 25(4 Pt 1):484-498.

3. Prinzen FW, Augustijn $\mathrm{CH}$, Arts T, Allessie MA, Reneman RS. Redistribution of myocardial fiber strain and blood flow by asynchronous activation. Am J Physiol 1990; 259(2 Pt 2):H300-308.

4. van Oosterhout MF, Prinzen FW, Arts T, Schreuder JJ, Vanagt WY, Cleutjens JP, Reneman RS. Asynchronous electrical activation induces asymmetrical hypertrophy of the left ventricular wall. Circulation 1998; 98(6):588-595.

5. Prinzen FW, Hunter WC, Wyman BT, McVeigh ER. Mapping of regional myocardial strain and work during ventricular pacing: experimental study using magnetic resonance imaging tagging. J Am Coll Cardiol 1999; 33(6):1735-1742.

6. van Oosterhout MF, Arts T, Bassingthwaighte JB, Reneman RS, Prinzen FW. Relation between local myocardial growth and blood flow during chronic ventricular pacing. Cardiovasc Res 2002; 53(4):831-840.

7. Prinzen FW, Van Oosterhout MF, Vanagt WY, Storm C, Reneman RS. Optimization of ventricular function by improving the activation sequence during ventricular pacing. Pacing Clin Electrophysiol 1998; 21(11 Pt 2):2256-2260.

8. Karpawich PP. Chronic right ventricular pacing and cardiac performance: the pediatric perspective. Pacing Clin Electrophysiol 2004; 27(6 Pt 2):844-849.

9. Manolis AS. The deleterious consequences of right ventricular apical pacing: time to seek alternate site pacing. Pacing Clin Electrophysiol 2006; 29(3):298-315.

10. Little WC, Reeves RC, Arciniegas J, Katholi RE, Rogers EW. Mechanism of abnormal interventricular septal motion during delayed left ventricular activation. Circulation 1982; 65(7):1486-1491.

11. Prinzen FW, Cheriex EC, Delhaas T, van Oosterhout MF, Arts T, Wellens HJ, Reneman RS. Asymmetric thickness of the left ventricular wall resulting from asynchronous electric activation: a study in dogs with ventricular pacing and in patients with left bundle branch block. Am Heart J 1995; 130(5):1045-1053.

12. Karpawich PP, Mital S. Comparative left ventricular function following atrial, septal, and apical single chamber heart pacing in the young. Pacing Clin Electrophysio/ 1997; 20(8 Pt 1):1983-1988.

13. Moak JP, Barron KS, Hougen TJ, Wiles HB, Balaji S, Sreeram N, Cohen MH, Nordenberg A, Van Hare GF, Friedman RA, Perez M, Cecchin F, Schneider DS, Nehgme RA, Buyon JP. Congenital heart block: development of late-onset cardiomyopathy, a previously underappreciated sequela. J Am Coll Cardiol 2001; 37(1):238-242. 
14. Tantengco MV, Thomas RL, Karpawich PP. Left ventricular dysfunction after long-term right ventricular apical pacing in the young. J Am Coll Cardiol 2001; 37(8):2093-2100.

15. Vernooy K, Verbeek XA, Peschar M, Prinzen FW. Relation between abnormal ventricular impulse conduction and heart failure. J Interv Cardio/ 2003; 16(6):557-562.

16. Thambo JB, Bordachar P, Garrigue S, Lafitte $S$, Sanders $P$, Reuter $S$, Girardot R, Crepin D, Reant $P$, Roudaut $R$, Jais $P$, Haissaguerre M, Clementy J, Jimenez $M$. Detrimental ventricular remodeling in patients with congenital complete heart block and chronic right ventricular apical pacing. Circulation 2004; 110(25):3766-3772.

17. Janousek J, Tomek V, Chaloupecky V, Gebauer RA. Dilated cardiomyopathy associated with dualchamber pacing in infants: improvement through either left ventricular cardiac resynchronization or programming the pacemaker off allowing intrinsic normal conduction. I Cardiovasc Electrophysiol 2004; 15(4):470-474.

18. Andersen HR, Nielsen JC, Thomsen PE, Thuesen L, Mortensen PT, Vesterlund T, Pedersen AK. Long-term follow-up of patients from a randomised trial of atrial versus ventricular pacing for sick-sinus syndrome. Lancet 1997; 350(9086):1210-1216.

19. Nielsen JC, Andersen HR, Thomsen PE, Thuesen L, Mortensen PT, Vesterlund T, Pedersen AK. Heart failure and echocardiographic changes during long-term follow-up of patients with sick sinus syndrome randomized to single-chamber atrial or ventricular pacing. Circulation 1998; 97(10):987-995.

20. Wilkoff BL, Cook JR, Epstein AE, Greene HL, Hallstrom AP, Hsia H, Kutalek SP, Sharma A. Dualchamber pacing or ventricular backup pacing in patients with an implantable defibrillator: the Dual Chamber and VVI Implantable Defibrillator (DAVID) Trial. JAMA 2002; 288(24):3115-3123.

21. Sweeney MO, Hellkamp AS, Ellenbogen KA, Greenspon AJ, Freedman RA, Lee KL, Lamas GA. Adverse effect of ventricular pacing on heart failure and atrial fibrillation among patients with normal baseline QRS duration in a clinical trial of pacemaker therapy for sinus node dysfunction. Circulation 2003; 107(23):2932-2937.

22. Zannad F, Huvelle E, Dickstein K, van Veldhuisen DJ, Stellbrink C, Kober L, Cazeau S, Ritter P, Maggioni AP, Ferrari R, Lechat P. Left bundle branch block as a risk factor for progression to heart failure. Eur J Heart Fail 2007; 9(1):7-14.

23. Cazeau S, Ritter P, Bakdach S, Lazarus A, Limousin M, Henao L, Mundler O, Daubert JC, Mugica J. Four chamber pacing in dilated cardiomyopathy. Pacing Clin Electrophysiol 1994; $17(11 \mathrm{Pt}$ 2):1974-1979.

24. Leclercq C, Cazeau S, Le Breton H, Ritter P, Mabo P, Gras D, Pavin D, Lazarus A, Daubert JC. Acute hemodynamic effects of biventricular DDD pacing in patients with end-stage heart failure. J Am Coll Cardiol 1998; 32(7):1825-1831.

25. Cazeau S, Leclercq C, Lavergne T, Walker S, Varma C, Linde C, Garrigue S, Kappenberger L, Haywood GA, Santini M, Bailleul C, Daubert JC. Effects of multisite biventricular pacing in patients with heart failure and intraventricular conduction delay. N Engl J Med 2001; 344(12):873-880. 
26. Abraham WT, Fisher WG, Smith AL, Delurgio DB, Leon AR, Loh E, Kocovic DZ, Packer M, Clavell AL, Hayes DL, Ellestad M, Trupp RJ, Underwood J, Pickering F, Truex C, McAtee P, Messenger J, the MSG. Cardiac Resynchronization in Chronic Heart Failure. N Engl J Med 2002; 346(24):18451853.

27. Gregoratos G, Abrams J, Epstein AE, Freedman RA, Hayes DL, Hlatky MA, Kerber RE, Naccarelli GV, Schoenfeld MH, Silka MJ, Winters SL, Gibbons RJ, Antman EM, Alpert JS, Gregoratos G, Hiratzka LF, Faxon DP, Jacobs AK, Fuster V, Smith SC, Jr. ACC/AHANASPE 2002 guideline update for implantation of cardiac pacemakers and antiarrhythmia devices: summary article: a report of the American College of Cardiology/American Heart Association Task Force on Practice Guidelines (ACC/AHA/NASPE Committee to Update the 1998 Pacemaker Guidelines). Circulation 2002; 106(16):2145-2161.

28. Linde C, Abraham WT, Gold MR, St John Sutton M, Ghio S, Daubert C. Randomized trial of cardiac resynchronization in mildly symptomatic heart failure patients and in asymptomatic patients with left ventricular dysfunction and previous heart failure symptoms. I Am Coll Cardiol 2008; 52(23):1834-1843.

29. St John Sutton MG, Plappert T, Abraham WT, Smith AL, DeLurgio DB, Leon AR, Loh E, Kocovic DZ, Fisher WG, Ellestad M, Messenger J, Kruger K, Hilpisch KE, Hill MR. Effect of cardiac resynchronization therapy on left ventricular size and function in chronic heart failure. Circulation 2003; 107(15):1985-1990.

30. Cleland JG, Daubert JC, Erdmann E, Freemantle N, Gras D, Kappenberger L, Tavazzi L. The effect of cardiac resynchronization on morbidity and mortality in heart failure. N Engl J Med 2005; 352(15):1539-1549.

31. Daubert JC, Leclercq C, Donal E, Mabo P. Cardiac resynchronisation therapy in heart failure: current status. Heart Fail Rev 2006; 11(2):147-154.

32. Donal E, Leclercq C, Linde C, Daubert JC. Effects of cardiac resynchronization therapy on disease progression in chronic heart failure. Eur Heart J 2006; 27(9):1018-1025.

33. Leclercq C, Cazeau S, Lellouche D, Fossati F, Anselme F, Davy JM, Sadoul N, Klug D, Mollo L, Daubert JC. Upgrading from single chamber right ventricular to biventricular pacing in permanently paced patients with worsening heart failure: The RD-CHF Study. Pacing Clin Electrophysiol 2007; 30 Suppl 1:S23-30.

34. Laurenzi F, Achilli A, Avella A, Peraldo C, Orazi S, Perego GB, Cesario A, Valsecchi S, De Santo T, Puglisi A, Tondo $C$. Biventricular upgrading in patients with conventional pacing system and congestive heart failure: results and response predictors. Pacing Clin Electrophysiol 2007; 30(9):1096-1104.

35. Shimano M, Tsuji Y, Yoshida Y, Inden Y, Tsuboi N, Itoh T, Suzuki H, Muramatsu T, Okada T, Harata S, Yamada T, Hirayama H, Nattel S, Murohara T. Acute and chronic effects of cardiac resynchronization in patients developing heart failure with long-term pacemaker therapy for acquired complete atrioventricular block. Europace 2007; 9(10):869-874. 
36. Vardas PE, Auricchio A, Blanc JJ, Daubert JC, Drexler H, Ector H, Gasparini M, Linde C, Morgado FB, Oto A, Sutton R, Trusz-Gluza M. Guidelines for cardiac pacing and cardiac resynchronization therapy. The Task Force for Cardiac Pacing and Cardiac Resynchronization Therapy of the European Society of Cardiology. Developed in collaboration with the European Heart Rhythm Association. Europace 2007; 9(10):959-998.

37. Young JB, Abraham WT, Smith AL, Leon AR, Lieberman R, Wilkoff B, Canby RC, Schroeder JS, Liem LB, Hall S, Wheelan K. Combined cardiac resynchronization and implantable cardioversion defibrillation in advanced chronic heart failure: the MIRACLE ICD Trial. JAMA 2003; 289(20):2685-2694.

38. Auricchio A, Stellbrink C, Block M, Sack S, Vogt J, Bakker P, Klein H, Kramer A, Ding J, Salo R, Tockman B, Pochet T, Spinelli J. Effect of pacing chamber and atrioventricular delay on acute systolic function of paced patients with congestive heart failure. The Pacing Therapies for Congestive Heart Failure Study Group. The Guidant Congestive Heart Failure Research Group. Circulation 1999; 99(23):2993-3001.

39. Auricchio A, Stellbrink C, Sack S, Block M, Vogt J, Bakker P, Huth C, Schondube F, Wolfhard U, Bocker D, Krahnefeld O, Kirkels $\mathrm{H}$. Long-term clinical effect of hemodynamically optimized cardiac resynchronization therapy in patients with heart failure and ventricular conduction delay. J Am Coll Cardiol 2002; 39(12):2026-2033.

40. Whinnett ZI, Davies JE, Willson K, Manisty CH, Chow AW, Foale RA, Davies DW, Hughes AD, Mayet J, Francis DP. Haemodynamic effects of changes in atrioventricular and interventricular delay in cardiac resynchronisation therapy show a consistent pattern: analysis of shape, magnitude and relative importance of atrioventricular and interventricular delay. Heart 2006; 92(11):1628-1634.

41. Perego GB, Chianca R, Facchini M, Frattola A, Balla E, Zucchi S, Cavaglia S, Vicini I, Negretto M, Osculati G. Simultaneous vs. sequential biventricular pacing in dilated cardiomyopathy: an acute hemodynamic study. Eur J Heart Fail 2003; 5(3):305-313.

42. Udink ten Cate FE, Breur JM, Cohen MI, Boramanand N, Kapusta L, Crosson JE, Brenner JI, Lubbers L, Friedman AH, Vetter VL, Meijboom EJ. Dilated cardiomyopathy in isolated congenital complete atrioventricular block: early and long-term risk in children. J Am Coll Cardiol 2001; 37(4):1129-1134.

43. Michaelsson M, Riesenfeld T, Jonzon A. Natural history of congenital complete atrioventricular block. Pacing Clin Electrophysiol 1997; 20(8 Pt 2):2098-2101. 
44. Epstein AE, DiMarco JP, Ellenbogen KA, Estes NA, 3rd, Freedman RA, Gettes LS, Gillinov AM, Gregoratos G, Hammill SC, Hayes DL, Hlatky MA, Newby LK, Page RL, Schoenfeld MH, Silka MJ, Stevenson LW, Sweeney MO, Smith SC, Jr., Jacobs AK, Adams CD, Anderson JL, Buller CE, Creager MA, Ettinger SM, Faxon DP, Halperin JL, Hiratzka LF, Hunt SA, Krumholz HM, Kushner FG, Lytle BW, Nishimura RA, Ornato JP, Page RL, Riegel B, Tarkington LG, Yancy CW. ACC/AHA/HRS 2008 Guidelines for Device-Based Therapy of Cardiac Rhythm Abnormalities: a report of the American College of Cardiology/American Heart Association Task Force on Practice Guidelines (Writing Committee to Revise the ACC/AHA/NASPE 2002 Guideline Update for Implantation of Cardiac Pacemakers and Antiarrhythmia Devices) developed in collaboration with the American Association for Thoracic Surgery and Society of Thoracic Surgeons. J Am Coll Cardiol 2008; 51(21):e1-62.

45. Kim JJ, Friedman RA, Eidem BW, Cannon BC, Arora G, Smith EO, Fenrich AL, Kertesz NJ. Ventricular function and long-term pacing in children with congenital complete atrioventricular block. J Cardiovasc Electrophysiol 2007; 18(4):373-377.

46. Karpawich PP, Rabah R, Haas JE. Altered cardiac histology following apical right ventricular pacing in patients with congenital atrioventricular block. Pacing Clin Electrophysiol 1999; 22(9):13721377.

47. Gebauer RA, Tomek V, Salameh A, Marek J, Chaloupecky V, Gebauer R, Matejka T, Vojtovic P, Janousek J. Predictors of left ventricular remodelling and failure in right ventricular pacing in the young. Eur Heart J 2009; 30(9):1097-1104.

48. Puggioni E, Brignole M, Gammage M, Soldati E, Bongiorni MG, Simantirakis EN, Vardas P, Gadler F, Bergfeldt L, Tomasi C, Musso G, Gasparini G, Del Rosso A. Acute comparative effect of right and left ventricular pacing in patients with permanent atrial fibrillation. J Am Coll Cardiol 2004; 43(2):234-238.

49. Wyman BT, Hunter WC, Prinzen FW, Faris OP, McVeigh ER. Effects of single- and biventricular pacing on temporal and spatial dynamics of ventricular contraction. Am J Physiol Heart Circ Physiol 2002; 282(1):H372-379.

50. Peschar M, de Swart H, Michels KJ, Reneman RS, Prinzen FW. Left ventricular septal and apex pacing for optimal pump function in canine hearts. J Am Coll Cardiol 2003; 41(7):1218-1226.

51. Vanagt WY, Verbeek XA, Delhaas T, Mertens L, Daenen WJ, Prinzen FW. The left ventricular apex is the optimal site for pediatric pacing: correlation with animal experience. Pacing Clin Electrophysiol 2004; 27(6 Pt 2):837-843.

52. Auricchio A. Pacing the left ventricle: does underlying rhythm matter? J Am Coll Cardiol 2004; 43(2):239-240.

53. Etienne Y, Mansourati J, Gilard M, Valls-Bertault V, Boschat J, Benditt DG, Lurie KG, Blanc JJ. Evaluation of left ventricular based pacing in patients with congestive heart failure and atrial fibrillation. Am J Cardiol 1999; 83(7):1138-1140, A1139. 
54. Touiza A, Etienne Y, Gilard M, Fatemi M, Mansourati J, Blanc JJ. Long-term left ventricular pacing: assessment and comparison with biventricular pacing in patients with severe congestive heart failure. J Am Coll Cardiol 2001; 38(7):1966-1970.

55. Vanagt WY, Prinzen FW, Delhaas T. Reversal of pacing-induced heart failure by left ventricular apical pacing. N Engl J Med 2007; 357(25):2637-2638.

56. Verbeek XA, Vernooy K, Peschar M, Cornelussen RN, Prinzen FW. Intra-ventricular resynchronization for optimal left ventricular function during pacing in experimental left bundle branch block. J Am Coll Cardiol 2003; 42(3):558-567.

57. Vernooy K, Verbeek XA, Cornelussen RN, Dijkman B, Crijns HJ, Arts T, Prinzen FW. Calculation of effective $\mathrm{V}$ interval facilitates optimization of $A V$ delay and $V V$ interval in cardiac resynchronization therapy. Heart Rhythm 2007; 4(1):75-82. 
Irene E. van Geldorp ${ }^{1,2}$

Kevin Vernooy ${ }^{1,2}$

Tammo Delhaas ${ }^{1,3}$

Martin H. Prins ${ }^{4}$

Harry J.G.M. Crijns ${ }^{1}$

Frits W. Prinzen ${ }^{2}$

Barbara Dijkman ${ }^{1}$

From the Departments of ${ }^{1}$ Cardiology and ${ }^{2}$ Physiology, Cardiovascular Research Institute Maastricht (CARIM), Maastricht University Medical Center, Maastricht, The Netherlands; ${ }^{3}$ Department of Pediatrics, Maastricht University Medical Center, Maastricht, The Netherlands; ${ }^{4}$ Departments of Clinical Epidemiology and Medical Technology Assessment (KEMTA), Maastricht University Medical Center, Maastricht, The Netherlands. 


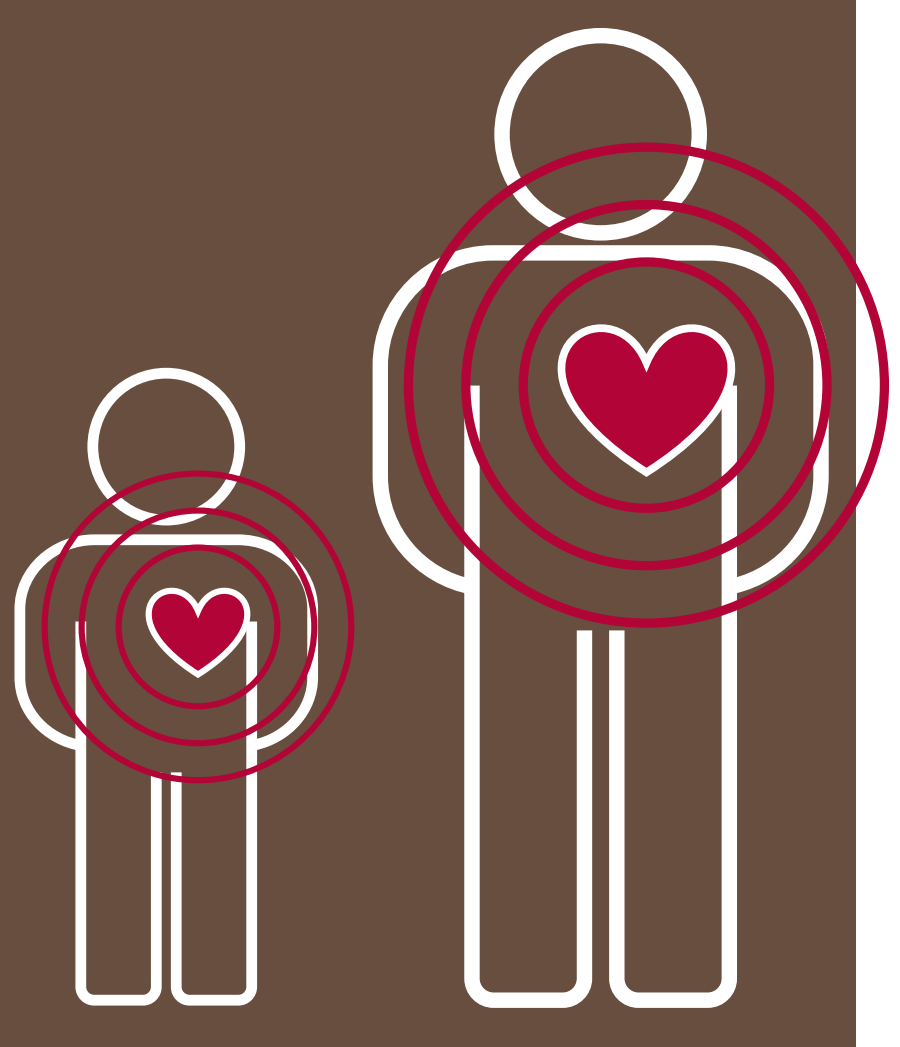

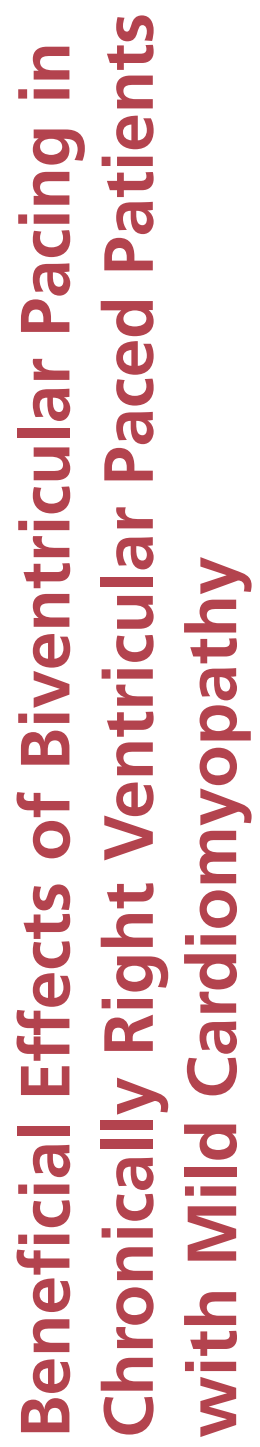




\section{Abstract}

Aims: To investigate whether cardiac resynchronisation therapy (CRT) by means of biventricular (BiV) pacing can improve left ventricular (LV) function, remodelling and clinical status in chronically right ventricular (RV) paced patients with mild cardiomyopathy.

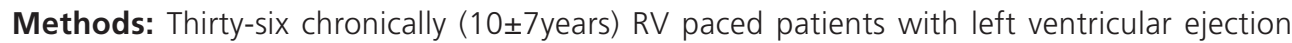
fraction $($ LVEF) $<40 \%$ or LVEDD $>55 \mathrm{~mm}$, without an established indication for CRT, were subjected to 6 months RV and BiV pacing in a patient-blinded, randomized crossover design. Treatment-effects of BiV pacing were evaluated for LV function, LV remodelling and clinical status.

Results: As compared with RV pacing, BiV pacing significantly improved LV function (LVEF $46 \pm 12 \%$ vs. $39 \pm 12 \%$ and LVFS $24 \pm 7 \%$ vs. $21 \pm 7 \%$ ) and reduced LV end-diastolic and endsystolic diameters and volumes (LVEDD $56 \pm 8 \mathrm{~mm}$ vs. $59 \pm 8 \mathrm{~mm}$, LVESD $43 \pm 8 \mathrm{~mm}$ vs. $47 \pm 9 \mathrm{~mm}$, LVEDV $132 \pm 65 \mathrm{ml}$ vs. $144 \pm 62 \mathrm{ml}$ and LVESV $77 \pm 56 \mathrm{ml}$ vs. $92 \pm 55 \mathrm{ml}$, respectively). In 19 patients (53\%) response to BiV pacing was clinically relevant, defined as LVESV reduction $>15 \%$. BiV pacing also significantly improved NYHA classification.

Conclusion: BiV pacing following chronic RV pacing may improve LV function and reverse LV remodelling in patients with relatively mild LV dysfunction or remodelling. Hence, upgrade to BiV pacing might be considered in chronically RV paced patients with mild cardiomyopathy. 


\section{INTRODUCTION}

In right ventricular (RV) pacing, the sequence of electrical activation resembles the activation pattern as in left bundle branch block (LBBB). ${ }^{1}$ This asynchronous electrical pattern is accompanied by abnormal dyssynchronous mechanical interactions within the left ventricle (LV). ${ }^{2,3}$ Evidence is increasing that LBBB as well as RV pacing, are associated with impairment of LV function, structural remodelling of the LV, and an increased risk for heart failure. ${ }^{4-9}$ Cardiac resynchronisation therapy (CRT) by means of biventricular (BiV) pacing, aims to reverse the deleterious effects that may originate from LV dyssynchrony. In the majority of patients with severe LV dysfunction and severe clinical heart failure associated with either LBBB, ${ }^{10-15}$ or RV pacing induced dyssynchrony, ${ }^{16,17}$ BiV pacing improves clinical presentation, reduces mortality, reverses LV remodelling and improves LV function. Therefore, BiV pacing is nowadays strongly recommended in patients with ventricular dyssynchrony (QRS $>120 \mathrm{~ms}$ ), severe LV dysfunction (LV ejection fraction (LVEF) $<35 \%$ ) and moderate to severe clinical heart failure (NYHA classification III-IV). ${ }^{18}$ The purpose of the present study was to investigate whether BiV pacing can improve LV function and can reverse LV remodelling in chronically RV paced patients with mild cardiomyopathy. The second aim was to investigate whether BiV pacing could improve, or at least maintain, clinical performance in those patients without severe heart failure.

\section{Methods}

\section{Study population and inclusion criteria}

From a database with \pm 1000 pacemaker patients in the Maastricht University Medical Centre, all patients with permanent pacemaker stimulation were screened for inclusion. Criteria for inclusion in the study were permanent RV pacing (>95\% paced beats) accompanied with echocardiographic signs of LV remodelling (defined as LV end-diastolic diameter (LVEDD) $>55 \mathrm{~mm}$ ) or LV dysfunction (defined as LVEF $<40 \%$ ). At least one of these echocardiographic signs had to be present despite stable pharmacological treatment and pacemaker programming aimed at maximal ventricular filling and minimal ventricular pacing. Inclusion was regardless of clinical symptoms of heart failure. Nevertheless, patients with severe LV dysfunction in combination with moderate to severe heart failure symptoms (i.e. LVEF $<35 \%$ and NYHA III-IV, respectively) were excluded from the current study, as they had an established indication for $\mathrm{CRT}^{18}$ Other criteria for exclusion were myocardial infarction or cardiac surgery within the previous six months, or non-cardiac conditions that could limit exercise capacity and life expectancy (within the duration of the study). 


\section{Study protocol}

The Institutional Review Board approved the protocol and all patients provided written informed consent at enrolment in the study (for study design see Figure 1). Patients underwent an upgrade to a BiV pacing device and had routine post-implant pacemaker evaluations. Post-implant all patients were BiV paced to detect and solve possible problems with pacing leads or pacemaker systems within the run-in period. Two to four weeks after implantation patients were randomly assigned to either RV or BiV pacing. At the same time-point individual optimization of the pacemaker settings was performed using echocardiography. The atrioventricular-delay in both RV and BiV pacing was optimized to the shortest delay that still provided maximal ventricular filling, assessed using pulsed Doppler analysis of the transmitral flow. Patients with permanent atrial fibrillation were exempt from the atrioventricular-delay procedure. Optimal interventricularinterval in BiV pacing was determined by maximizing the aortic velocity-time-integral. After 6 months of either BiV pacing or RV pacing, patients crossed over to the other pacing configuration for which the same optimization procedure was performed as at randomization. Thereafter, they were paced in the other pacing configuration for another 6 months. Patients were blinded for pacemaker configurations. Changes in pacing mode were performed by an independent physician, using an external programmer. Clinical and echo-cardiographic

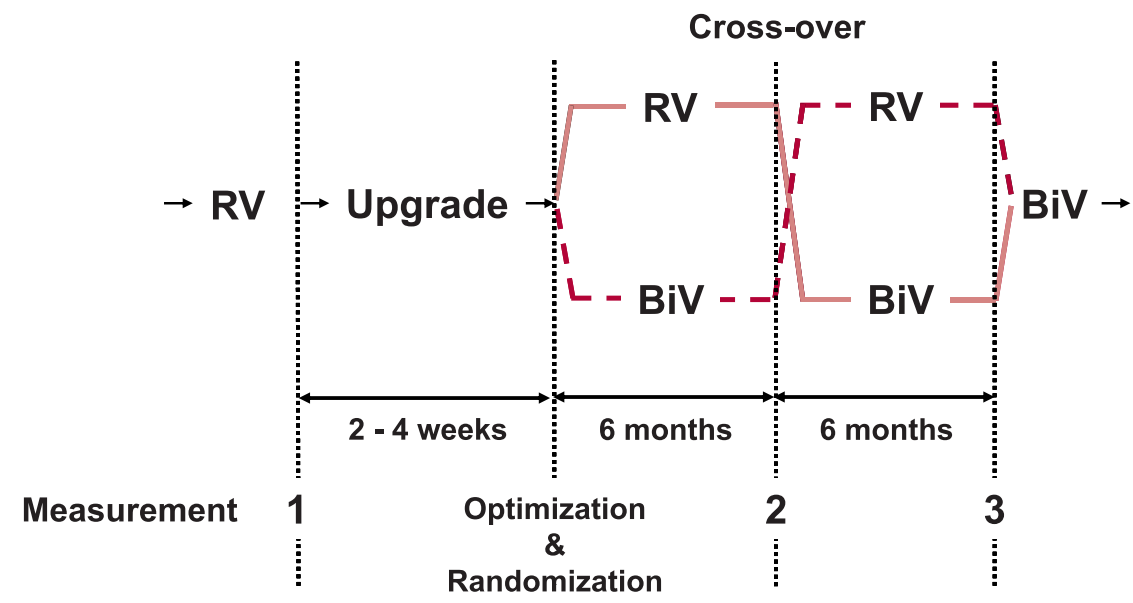

\section{Figure 1 - Study design.}

Subsequent to inclusion and baseline evaluation after chronic right ventricular (RV) pacing, patients underwent an upgrade procedure to a biventricular (BiV) pacing device and a run-in period before optimization of pacing settings. Thereafter patients were randomized to either BiV pacing or RV pacing, followed by a crossover to the other mode. At the end of each 6 months pacing period left ventricular (LV) function, LV remodelling and clinical status were evaluated. 
evaluations were performed before enrolment in the study (prior to upgrade procedure; measurement 1), and after the first and second phases (6 and 12 months after randomization; measurement 2 and 3, respectively).

\section{Clinical evaluation}

Symptoms of heart failure were classified according to the NYHA classification for heart failure. For further assessment of clinical status, a 12-lead electrocardiogram and an exercise tolerance test (i.e. treadmill-test, 6 min hall-walk distance, or bicycle-test) were performed. Approximate oxygen cost during exercise testing was derived from the peak work rate achieved by the patient and was defined in metabolic equivalents (METs). ${ }^{19}$ The self-administered "Minnesota Living with Heart Failure Questionnaire" was used for scoring the quality-of-life on a scale from 0 (best) to 105 (worst). ${ }^{20}$

\section{Echocardiographic evaluation}

All measurements were performed according to the guidelines of the American Society of Echocardiography. An experienced physician performed the echocardiographic evaluation using Philips Sonos 5500 or Philips iE 33 (Philips Medical Systems, The Netherlands) with 1.8-3.5-MHz transducers. Standard 2D and Doppler data from three consecutive beats were digitally stored for offline analysis with a customized software package (Xcelera, Philips Medical Systems, The Netherlands). During post-processing a single reviewer (K.V.) systematically measured LV end-diastolic (LVEDD) and LV end-systolic diameters (LVESD) in parasternal long-axis views. End-diastolic and endsystolic LV volumes (LVEDV and LVESV, respectively) and LVEF were calculated from the apical twoand four-chamber images with Simpson's biplane method. ${ }^{21}$ Additionally, LV fractional shortening (LVFS) was used as a parameter for LV pump function [LVFS=(LVEDD-LVESD)/LVEDD]. Using color-flow Doppler in the apical four-chamber view, mitral regurgitation was assessed and its severity was semiquantitatively graded on a scale from 0 (no regurgitation) to 4 (severe regurgitation). ${ }^{22}$ The time delay between the onset of aortic and pulmonic flow was used to define interventricular asynchrony. The time difference in onset of systolic motion between septum and LV lateral wall, determined using pulse wave tissue Doppler, was used as a measure of intraventricular dyssynchrony.

\section{Pacemaker implantation}

All patients were implanted with Guidant (St.Paul, MN, USA) BiV pacemaker systems (B.D.). When indicated an implantable cardioverter-defibrillator (ICD) with an additional LV pacing lead was implanted. LV pacing leads were inserted by a transvenous approach via the coronary sinus into either the lateral or postero-lateral vein whenever possible. Well functioning right atrial and apical $\mathrm{RV}$ leads of the prior pacemaker system were used for the upgraded system. In case of ICD implantation an RV coil lead was placed in the RV apex. After the upgrade procedure, lead positions of RV- and LV leads were determined from chest X-ray films (posterior-anterior and lateral view). 


\section{Statistical analysis}

Statistical data-analysis was performed (M.P., I.v.G.) using SPSS version 16.0 (SPSS Inc., Chicago, IL, USA). A two-tailed $P$-value of $<0.05$ was considered significant. Reduction in LVESV was considered as major efficacy variable for the study. The smallest difference with clinical significance was considered to be a change in LVESV of $>10 \%$ with BiV pacing as compared to RV pacing. To reach over $80 \%$ power with an estimated $10 \%$ loss of patients during follow up, the study had to include 37 patients (type 1 error: 0.05, 2-tailed; and type 2 error: 0.10). Baseline characteristics at study enrolment were assessed. Comparisons of baseline characteristics between both randomization groups were performed using either unpaired Student $t$-test for continuous or $X^{2}$ test for discrete variables. The efficiency (described in terms of treatmenteffects) of BiV pacing treatment as compared to RV pacing, was assessed from crossover data using one-way analysis of variance (ANOVA) for repeated measures. The stimulation mode (RV versus BiV pacing) was defined as within-subjects factor. To allow for testing on treatment-period interaction (carry-over effect), the randomization sequence ( $R V \rightarrow B i V$ versus $B i V \rightarrow R V$ ) was entered as a between-groups factor. Responders were defined as patients with an LVESVdifference (RV paced LVESV versus BiV paced LVESV) $>15 \%$ in favour of BiV pacing, since this degree of LVESV reduction has been used as a cut-off value for response in other trials. ${ }^{23-26}$ Comparison between responders and non-responders was performed with the use of unpaired Student $t$-test or $X^{2}$ test. Pearson's product-moment correlation coefficient was used to measure the strength of relationship between treatment-effects and baseline LVEF, LVESV, interventricular asynchrony and intraventricular dyssynchrony during RV pacing, the duration of RV pacing prior to, and the age at upgrade. Additionally, to detect possible predictors for the response to BiV pacing analysis of covariance (ANCOVA) was performed on the treatment-effect of BiV pacing on LVESV. In this ANCOVA analysis NYHA classification at inclusion, QRS duration in RV pacing, LV lead position, and baseline echocardiographic characteristics were defined as covariates (independent variables). 


\section{RESULTS}

\section{Study population}

From the entire pacemaker population 706 patients were paced in VVI or DDD mode. Within this group 93 patients were eligible. However, eleven of them had an indication for CRT following the guidelines and were therefore excluded from this study. Two patients recently suffered myocardial infarction, and in 31 patients exercise capacity and life expectancy were limited due to non-cardiac causes. Among the remaining 49 patients matching all study-criteria, 40 patients provided written informed consent. Thereafter, one patient was withdrawn from the study, as he preferred to have no replacement and upgrade of his pacemaker system prior to end-of-life of the system. Two patients were excluded from the study because transvenous LV lead implantation failed and surgical intervention was unfavourable. In the period between October 2004 and October 2006, 37 patients were enrolled after successful implantation of a BiV pacing device (ICD; $n=12)$. After the upgrade procedure and the run-in period, 18 patients were randomized to receive $\mathrm{RV}$ pacing in the first 6 months (group $\mathrm{A}$ ) and to crossover to BiV pacing for the second period of 6 months. The other 19 patients (group B) were randomized to both pacing treatments in reversed order (BiV pacing first and RV pacing thereafter). In one patient from group A, LV function and remodelling worsened considerably during RV pacing in the first period. Within three months after programming to BiV pacing he died from end-stage heart failure. Since paired data (BiV versus RV) from this patient were not available, this case was excluded from analysis. From the patients included, nineteen patients met both echocardiographic inclusion criteria (LVEF $<40 \%$ and LVEDD $>55 \mathrm{~mm}$ ). The other patients had echocardiographic signs of either remodelling (LVEDD $>55 \mathrm{~mm}(n=13)$ ), or impaired LV function (LVEF $<40 \%(n=4))$. All subject characteristics at study enrolment are summarized in Table 1. On average, patients had relatively mild LV dysfunction (LVEF 36 $\pm 10 \%$ ) and they were without

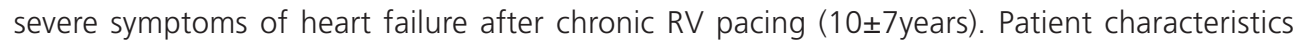
were not significantly different between both randomization groups.

\section{Complications}

In one patient the LV lead was placed epicardially via a minimal invasive thoracotomy after transvenous implantation of the lead failed. In another patient the initial procedure was discontinued because of a coronary sinus dissection, and re-intervention was performed one month later. In three other patients re-intervention was needed because of, stimulation of the diaphragm by the LV lead, RV lead dislodgement $(n=1)$ or impending LV lead dislodgement $(n=1)$. Minor complications were pocket haematoma $(n=3)$ and temporary stimulation of the diaphragm $(n=1)$. All complications mentioned were solved before pacemaker optimization and randomization. 


\section{Chapter 3}

Table 1 - Patient characteristics at baseline

\begin{tabular}{|c|c|c|c|c|}
\hline & $\begin{array}{l}\text { Total } \\
(n=36)\end{array}$ & $\begin{array}{l}\text { Group A } \\
(n=17)\end{array}$ & $\begin{array}{l}\text { Group B } \\
(n=19)\end{array}$ & $p$-value \\
\hline Gender (M/F) & $28 / 8$ & $13 / 4$ & $15 / 4$ & 0.858 \\
\hline Age (years) & $65 \pm 10$ & $67 \pm 10$ & $64 \pm 11$ & 0.374 \\
\hline History of RV pacing (years) & $10 \pm 7$ & $9 \pm 7$ & $11 \pm 7$ & 0.562 \\
\hline Aetiology of pacing indication & & & & 0.790 \\
\hline - Spontaneous AV-block, n (\%) & $17(47 \%)$ & $7(41 \%)$ & $10(53 \%)$ & \\
\hline - Surgically induced AV-block, n (\%) & $3(8 \%)$ & $2(12 \%)$ & $1 \quad(5 \%)$ & \\
\hline - His-ablation (in permanent atrial fibrillation), $n(\%)$ & $11(31 \%)$ & $6(35 \%)$ & $5 \quad(26 \%)$ & \\
\hline - Brady/Tachy syndrome (in atrial fibrillation), n (\%) & $5(14 \%)$ & $2(12 \%)$ & $3(16 \%)$ & \\
\hline \multicolumn{5}{|l|}{ Co-morbidity } \\
\hline - Coronary artery disease, $\mathrm{n}(\%)$ & $13(36 \%)$ & $8(47 \%)$ & $5 \quad(26 \%)$ & 0.196 \\
\hline - Atrial fibrillation (permanent), n (\%) & $19(15) \quad(53 \%)$ & $7(7) \quad(41 \%)$ & $12(8) \quad(63 \%)$ & 0.187 \\
\hline - Hypertension, n (\%) & $6(17 \%)$ & $4(24 \%)$ & $2(11 \%)$ & 0.296 \\
\hline - Diabetes mellitus, n (\%) & $3(8 \%)$ & $1(6 \%)$ & $2(11 \%)$ & 0.615 \\
\hline NYHA classification & & & & 0.617 \\
\hline - Class I, n (\%) & $9(25 \%)$ & $4(24 \%)$ & $5(26 \%)$ & \\
\hline - Class II, n (\%) & $19(53 \%)$ & $10(59 \%)$ & $9 \quad(47 \%)$ & \\
\hline - Class III, n (\%) & $8(22 \%)$ & $3(18 \%)$ & $5 \quad(26 \%)$ & \\
\hline RV paced QRS duration (ms) & $195 \pm 26$ & $196 \pm 29$ & $193 \pm 23$ & 0.078 \\
\hline $\operatorname{LVEDD}(\mathrm{mm})$ & $60 \pm 7$ & $59 \pm 7$ & $60 \pm 7$ & 0.698 \\
\hline $\operatorname{LVESD}(\mathrm{mm})$ & $48 \pm 8$ & $48 \pm 8$ & $48 \pm 9$ & 0.984 \\
\hline LVEDV (ml) & $155 \pm 72$ & $147 \pm 63$ & $163 \pm 80$ & 0.520 \\
\hline LVESV (ml) & $104 \pm 64$ & $98 \pm 61$ & $109 \pm 69$ & 0.633 \\
\hline $\operatorname{LVEF}(\%)$ & $36 \pm 10$ & $36 \pm 11$ & $36 \pm 9$ & 0.938 \\
\hline LVFS (\%) & $20 \pm 6$ & $20 \pm 6$ & $21 \pm 6$ & 0.552 \\
\hline
\end{tabular}

AV-block = atrioventricular block; LVEDD and LVESD = left ventricular end-diastolic and end-systolic diameter, respectively; LVEDV and LVESV = left ventricular end-diastolic and end-systolic volume; LVEF = left ventricular ejection fraction; LVFS = left ventricular fractional shortening; RV = right ventricular. 

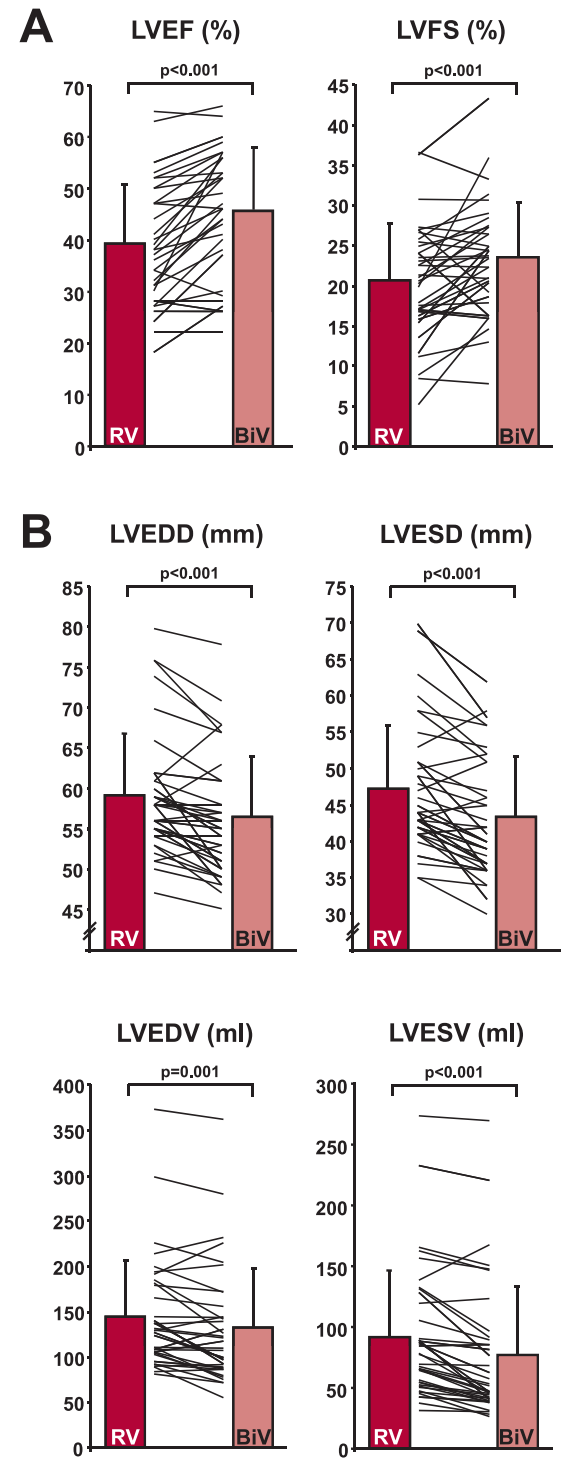

\section{Biventricular pacing improves left ventricular function and reverses left ventricular remodelling}

There was no significant effect of the treatment sequence $\quad(R V \rightarrow$ BiV vs. BiV $\rightarrow R V)$, as no interaction between randomization sequence and within-subjects factor could be detected for any of the parameters evaluated (no carry-over effect). Thus, treatment-effects of BiV pacing could be calculated from the BiV paced and RV paced measurements previous to and 6 months after cross-over (baseline measure-ments are not included in this analysis). Figure 2 shows the results on echocardio-graphic measurements of 6 months BiV pacing versus 6 months RV pacing. The positive treatment-effects of BiV pacing in LVEF ( $+7 \% ; p<0.001)$ and LVFS (+3\%; $p=0.001)$ are indicative for a significantly better LV function with BiV pacing as compared to RV pacing. LV dilatation was significantly less in BiV pacing as compared to RV pacing, as expressed by smaller diameters and volumes in BiV pacing as compared with RV pacing (LVEDD: $-4 \%$, $p<0.001$; LVESD: $-8 \%, p<0.001$; LVEDV: $-9 \%$, $p=0.001$; LVESV: $-19 \%, p<0.001$, respectively). The severity-grade of mitral regurgitation was similar in both pacing configurations $(1.3 \pm 0.9$ and $1.1 \pm 0.7$ during RV and BiV pacing, respectively; $p=0.107)$. In 19 patients (53\%) the LVESV-difference (RV paced LVESV vs. BiV paced LVESV) was $>15 \%$ in favour of BiV pacing. These patients are referred to as 'responders'. Only one

Figure 2 - Left ventricular function and remodelling.

Comparison of left ventricular (LV) function (A) and LV remodelling (B) after 6 months biventricular (BiV) pacing vs. 6 months right ventricular (RV) pacing. LVEF = LV ejection fraction; LVFS = LV fractional shortening; LVEDD = LV end-diastolic diameter; LVESD = LV end-systolic diameter; LVEDV = LV end-diastolic volume; LVESV = LV end-systolic volume. 
patient (3\%) was defined as an 'adverse-responder' as in this patient the LVESV difference between both pacing-modes was $>15 \%$ in favour of RV pacing. In 20 patients (56\%) BiV pacing was beneficial for LV function indicated by a positive treatment-effect in LVEF $>5 \%$. In none of the patients, LVEF was adversely influenced $>5 \%$ by BiV pacing. In the responders the mean LVESV reduction (LVESV treatment-effect: $-32 \pm 10 \%$ ) was paralleled by a significantly larger treatmenteffect in LVEF as compared with non-responders $(+9 \pm 7 \%$ vs. $+4 \pm 5 \%$, respectively; $p=0.030)$ and the number of patients with LVEF-treatment-effect $>5 \%$ was 14 (74\%) in the responder-group.

\section{Clinical outcome}

NYHA classification was significantly better $(p=0.007)$ after BiV pacing as compared with RV pacing (Table 2). In six (17\%) patients NYHA classification was one class less in BiV pacing (NYHA II vs. NYHA III $(n=2)$ and NYHA I vs. NYHA II $(n=4)$ for BiV vs. RV pacing, respectively). In one patient (3\%) the difference was even two NYHA classification levels in favour of BiV pacing (NYHA I in BiV pacing vs. NYHA III in RV pacing). The mean LVESV reduction in all these seven patients was $19 \pm 22 \%$; four patients were responders (LVESV reduction $>15 \%$ ) and one patient was the 'adverse-responder' (LVESV increase $>15 \%$ ). Quality-of-life-scores and quantity of METs as derivative of performance on exercise testing were not significantly different during BiV pacing and RV pacing (Table 2).

\section{Determinants of response to biventricular pacing}

No evident determinant for the response to BiV pacing was found. The degree of difference between RV paced and BiV paced LVESV was neither affected by baseline LVESV (Pearson's $R=-0.5$, ANCOVA $p=0.163)$, nor by baseline $\operatorname{LVEF}(R=0.3 ; p=0.506)$ (Figure 3$)$. Also, the duration of chronic $R V$ pacing (10 1 7years) prior to enrolment in the current study $(R=0.1 ; p=0.777)$, and patients' age at upgrade $(R<0.1 ; p=0.308)$ did not affect the treatment-effect on LVESV. LV lead-tips were located at the lateral free wall ( $n=17)$, posterior free wall $(n=10)$, anterior free wall $(n=2)$, lateral-base $(n=2)$, posterior-base $(n=3)$ and anterior-base $(n=2)$. The position of the LV lead-tip was not a significant covariate for the response to BiV pacing $(p=0.596)$. The duration of the QRS complex was significantly shorter $(-16 \%$, $p<0.001)$ during BiV pacing than during RV pacing $(163 \pm 19 \mathrm{~ms}$, range $130-200 \mathrm{~ms}$ vs. $195 \pm 26 \mathrm{~ms}$, range 140-240ms). However, baseline QRS duration and measurements of interventricular- and LV intraventricular dyssynchrony could not predict the response to BiV pacing $(R=0.2$ and $p=0.802 ; R=-$ 0.2 and $p=0.633$; and $R=-0.1$ and $p=0.523$, respectively). The response to BiV pacing was not influenced by the NYHA classification at baseline $(p=0.271)$, nor by the presence of atrial fibrillation as a comorbidity $(p=0.501)$.

\section{Patients in NYHA I and II, subgroup analysis}

In patients with mild symptoms of heart failure (NYHA I and II, n=28) the treatment-effects of BiV pacing were similar to compared with the entire study cohort. In this subgroup, mean LVESV 
Table 2 - Clinical outcome

\begin{tabular}{lccc} 
& RV pacing & BiV pacing & p-value \\
\hline NYHA classification & & & 0.007 \\
- Class I, $n(\%)$ & $11(31 \%)$ & $16(44 \%)$ & \\
- Class II, $n(\%)$ & $18(50 \%)$ & $16(44 \%)$ & \\
- Class III, $n(\%)$ & $7(19 \%)$ & $4(11 \%)$ & \\
Quality-of-life score (total) & $30 \pm 24$ & $27 \pm 23$ & 0.427 \\
Exercise test (METs) & $9 \pm 4$ & $10 \pm 3$ & 0.205 \\
\hline BiV = biventricular; METs = metabolic equivalents; RV = right ventricular. & &
\end{tabular}
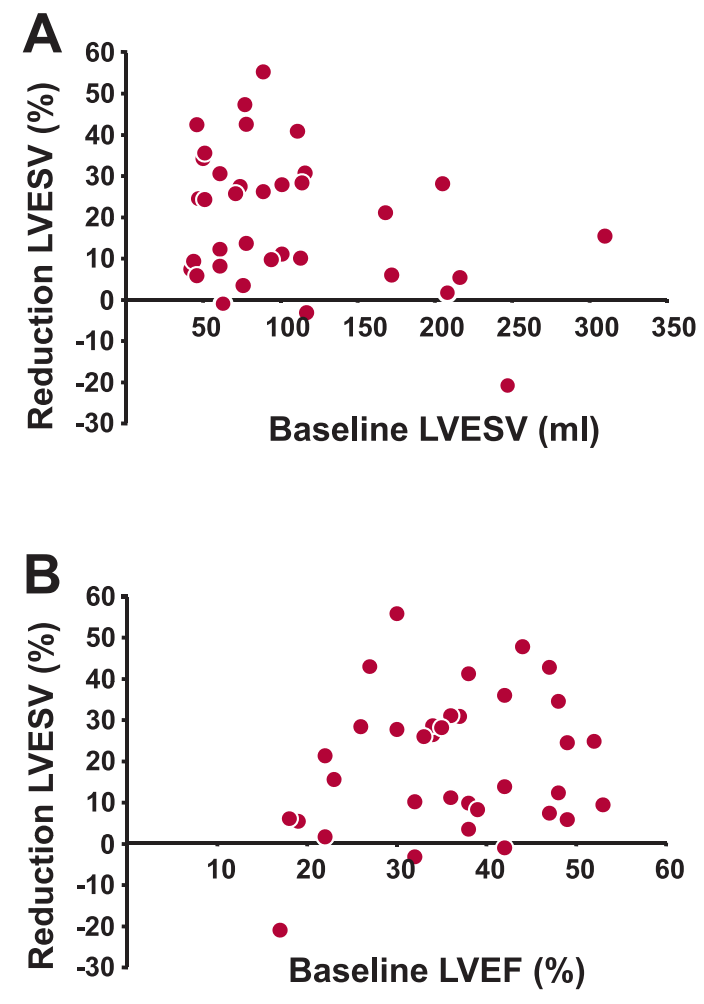

Figure 3 - Response to biventricular pacing.

Percentage reduction in left ventricular end-systolic volume (LVESV) after biventricular pacing plotted against the baseline LVESV (A) and baseline left ventricular ejection fraction (LVEF) (B). 
reduction was $19 \%(p<0.001)$ and LVESV reduction was $>15 \%$ in 15 patients $(54 \%)$. All parameters of remodelling were smaller in BiV pacing as compared with RV pacing: LVEDD $56 \pm 7 \mathrm{~mm}$ vs. $58 \pm 7 \mathrm{~mm}(4 \%, \mathrm{p}=0.002)$, LVESD $43 \pm 8 \mathrm{~mm}$ vs. $46 \pm 9 \mathrm{~mm}(8 \%, p<0.001)$, LVEDV $124 \pm 53 \mathrm{ml}$ vs. $134 \pm 49 \mathrm{ml}(8 \%, \mathrm{p}=0.025)$ and LVESV $68 \pm 47 \mathrm{ml}$ vs. $83 \pm 47 \mathrm{ml}(19 \%, p<0.001)$. Also regarding LV function, BiV pacing was better in comparison with RV pacing, indicated by a higher mean LVEF $(48 \pm 12 \%$ vs. $41 \pm 12 \%, p<0.001)$ and higher LVFS $(24 \pm 7 \%$ vs. $21 \pm 8 \%$, $\mathrm{p}=0.004)$. In 16 patients (57\%) from this subgroup the difference between RV and BiV paced LVEF was $>5 \%$ in favour of BiV pacing.

\section{Discussion}

The present study shows that BiV pacing following chronic RV pacing improves LV function, reverses LV remodelling and improves NYHA classification in patients with cardiomyopathy associated with RV pacing, although not having an established indication for CRT.

\section{Biventricular pacing in asymptomatic and mildly symptomatic patients with relatively mild left ventricular dysfunction}

On average, patients from this study had less severe LV dysfunction and LV remodelling, as well as fewer clinical symptoms of heart failure, as compared with patients in other studies on CRT. Even though patients in the present study had these better conditions, response to BiV pacing was evident with clinically relevant reverse remodelling (LVESV reduction $>15 \%$ ) in 53\% of the patients, and improvement in LV function in the majority of them. The finding that BiV pacing is beneficial in mildly symptomatic and even in asymptomatic patients (NYHA I-II subgroup) is supported by earlier investigations. ${ }^{27-29}$ The present study shows that improvement of LV function and reversion of LV remodelling after BiV pacing may even occur in patients with less severe LV dysfunction (LVEF $36 \pm 10 \%$ ) as compared with other studies (i.e., REVERSE study LVEF $27 \pm 7 \%$ ). ${ }^{27-29}$ Furthermore, the response to BiV pacing does not seem to be related to the severity of either LV dysfunction or LV remodelling.

\section{Biventricular pacing and clinical status}

In patients with severe heart failure BiV pacing has been shown to be beneficial regarding clinical status by many studies. ${ }^{13,16,17}$ The present study shows no evident benefit of BiV pacing in exercise performance or quality of life, despite significant beneficial treatment-effects of BiV pacing on LV function and remodelling. This finding might be explained by the fact that the parameters used were probably too rough to define modest benefits in those patients with a good clinical condition, leaving not much room for clinical improvement. Nevertheless, NYHA classification was improved by BiV pacing. Though, the clinical benefit of BiV pacing was not always paralleled by echocardiographic benefit. 


\section{Biventricular pacing in patients with a history of chronic right ventricular pacing}

Although in most studies benefits of CRT are shown in patients with dyssynchrony due to an 'intrinsic' LBBB (ischemic or idiopathic), in the current study beneficial treatment-effects of BiV pacing are shown in patients with RV pacing-induced dyssynchrony (paced LBBB). Improvement in LV function and reversion of LV remodelling are described for BiV upgrade in chronically RV paced patients by other studies too, ${ }^{2}, 16,17,30$ but the history of chronic RV pacing was longer in the current study (10 \pm 7years). Delnoy et al. have shown similar clinical and echocardiographic improvement when CRT was applied in patients with preceding chronic RV pacing compared with patients with native LBBB. ${ }^{31}$ In the current study the response to BiV pacing does not seem to be related to the number of years of chronic RV pacing prior to the upgrade. To appreciate these findings, it is important to keep in mind that also in LBBB patients, dyssynchrony may have existed for several years before the presence of symptoms becomes the final indication for 'de novo CRT'. As CRT restores LV electrical and mechanical synchrony, and therefore improves LV function, it seems reasonable that response may be independent of aetiology and time-span of the underlying dyssynchronous activation pattern.

\section{Implications of results}

To date, the presence of severe LV dysfunction (LVEF <35\%) and moderate to severe clinical heart failure (NYHA classification III-IV) are widely used as criteria for CRT. Although the new American guidelines are less tenacious to the presence of clinical heart failure symptoms, severe LV dysfunction is still a strict criterion: it is advised to consider CRT in slightly symptomatic patients (NYHA II) only if LVEF $<35 \% .{ }^{32}$ Improvement of clinical condition and improvement in echocardiographic parameters in patients with mild heart failure suggest that CRT prevents or slows the progression to severe heart failure. In the prevention of LV dysfunction, LV remodelling and heart failure, it might be valuable to start BiV pacing in patients with an indication for permanent ventricular stimulation. However, the complication rate and unnecessary costs are important issues in a mildly symptomatic patient cohort and make (upgrade to) BiV pacing in every patient with a conventional pacemaker indication or an LBBB unfavourable. Nevertheless, our suggestion is to monitor LV function routinely and to carefully consider (upgrade to) BiV pacing from the moment that echocardiographic signs of remodelling or deterioration of LV function occur, whereas disregarding NYHA classification in this concern.

\section{Limitations of the study}

As this was a single-centre study, the study was limited to a relatively small number of patients. Nonetheless, in a crossover-design every patient acts as his/her own control, so paired analysis can be performed and fewer patients are needed to reach a powered level of significance. Using a crossover-design, placebo effects of study-inclusion and pacemaker upgrade to a BiV device 
can be excluded, because data from the crossover-phase were used for the assessment of treatment-effects. Furthermore, patients were blinded for pacemaker configurations. Conversely, in a single blind trial unintended bias by investigators cannot be totally excluded. To minimize this bias the investigators were unaware of the results of previous measurements in the individual patient.

\section{Conclusion}

We conclude that BiV pacing following chronic RV pacing may improve LV function and reverse LV remodelling even in patients with relatively mild LV dysfunction or remodelling, and no severe clinical symptoms of heart failure. In addition, in these patients clinical status can be improved by BiV pacing. Hence, upgrade to BiV pacing might be considered in patients with mild cardiomyopathy associated with chronic RV pacing, even though they have no established indication for CRT following current guidelines.

\section{Funding}

This work was supported by Boston Scientific Corporation (Natick, MA, USA).

\section{Acknowledgements}

We gratefully acknowledge the patients who participated in this study. We would also like to thank our echocardiographist Jos Habets BSC, for performing all echocardiographic investigations in the study.

\section{Disclosures}

Frits Prinzen is consultant to Medtronic Inc. (Minneapolis, MN, USA) and receives research grants from Medtronic Inc. (Minneapolis, MN, USA), Boston Scientific Corp. (Natick, MA, USA) and EBR Systems (Sunnyvale, CA, USA). 


\section{REFERENCES}

1. Vassallo JA, Cassidy DM, Miller JM, Buxton AE, Marchlinski FE, Josephson ME. Left ventricular endocardial activation during right ventricular pacing: effect of underlying heart disease. J Am Coll Cardiol 1986; 7(6):1228-1233.

2. Witte KK, Pipes RR, Nanthakumar K, Parker JD. Biventricular pacemaker upgrade in previously paced heart failure patients-improvements in ventricular dyssynchrony. J Card Fail 2006; 12(3):199-204.

3. Lumens J, Delhaas T, Kirn B, Arts T. Modeling ventricular interaction: a multiscale approach from sarcomere mechanics to cardiovascular system hemodynamics. Pac Symp Biocomput 2008:378389.

4. Manolis AS. The deleterious consequences of right ventricular apical pacing: time to seek alternate site pacing. Pacing Clin Electrophysiol 2006; 29(3):298-315.

5. Moak JP, Hasbani K, Ramwell C, Freedenberg V, Berger JT, DiRusso G, Callahan P. Dilated cardiomyopathy following right ventricular pacing for AV block in young patients: resolution after upgrading to biventricular pacing systems. J Cardiovasc Electrophysiol 2006; 17(10):10681071.

6. Sweeney MO, Hellkamp AS, Ellenbogen KA, Greenspon AJ, Freedman RA, Lee KL, Lamas GA. Adverse effect of ventricular pacing on heart failure and atrial fibrillation among patients with normal baseline QRS duration in a clinical trial of pacemaker therapy for sinus node dysfunction. Circulation 2003; 107(23):2932-2937.

7. Sweeney MO, Prinzen FW. A new paradigm for physiologic ventricular pacing. J Am Coll Cardiol 2006; 47(2):282-288.

8. Thambo JB, Bordachar P, Garrigue S, Lafitte $S$, Sanders $P$, Reuter $S$, Girardot R, Crepin D, Reant $P$, Roudaut $R$, Jais $P$, Haissaguerre M, Clementy J, Jimenez M. Detrimental ventricular remodeling in patients with congenital complete heart block and chronic right ventricular apical pacing. Circulation 2004; 110(25):3766-3772.

9. Vernooy K, Dijkman B, Cheriex EC, Prinzen FW, Crijns HJ. Ventricular remodeling during longterm right ventricular pacing following His bundle ablation. Am J Cardiol 2006; 97(8):1223-1227.

10. Abraham WT, Fisher WG, Smith AL, Delurgio DB, Leon AR, Loh E, Kocovic DZ, Packer M, Clavell AL, Hayes DL, Ellestad M, Trupp RJ, Underwood J, Pickering F, Truex C, McAtee P, Messenger J, the MSG. Cardiac Resynchronization in Chronic Heart Failure. N Engl J Med 2002; 346(24):1845-1853.

11. Cazeau S, Leclercq C, Lavergne T, Walker S, Varma C, Linde C, Garrigue S, Kappenberger L, Haywood GA, Santini M, Bailleul C, Daubert JC. Effects of multisite biventricular pacing in patients with heart failure and intraventricular conduction delay. N Engl J Med 2001; 344(12):873-880. 
12. Cleland JG, Daubert JC, Erdmann E, Freemantle N, Gras D, Kappenberger L, Tavazzi L. The effect of cardiac resynchronization on morbidity and mortality in heart failure. N Engl J Med 2005; 352(15):1539-1549.

13. Daubert JC, Leclercq C, Donal E, Mabo P. Cardiac resynchronisation therapy in heart failure: current status. Heart Fail Rev 2006; 11(2):147-154.

14. Donal E, Leclercq C, Linde C, Daubert JC. Effects of cardiac resynchronization therapy on disease progression in chronic heart failure. Eur Heart J 2006; 27(9):1018-1025.

15. St John Sutton MG, Plappert T, Abraham WT, Smith AL, DeLurgio DB, Leon AR, Loh E, Kocovic DZ, Fisher WG, Ellestad M, Messenger J, Kruger K, Hilpisch KE, Hill MR. Effect of cardiac resynchronization therapy on left ventricular size and function in chronic heart failure. Circulation 2003; 107(15):1985-1990.

16. Leclercq C, Cazeau S, Lellouche D, Fossati F, Anselme F, Davy JM, Sadoul N, Klug D, Mollo L, Daubert JC. Upgrading from single chamber right ventricular to biventricular pacing in permanently paced patients with worsening heart failure: The RD-CHF Study. Pacing Clin Electrophysiol 2007; 30 Suppl 1:S23-30.

17. Laurenzi F, Achilli A, Avella A, Peraldo C, Orazi S, Perego GB, Cesario A, Valsecchi S, De Santo T, Puglisi A, Tondo C. Biventricular upgrading in patients with conventional pacing system and congestive heart failure: results and response predictors. Pacing Clin Electrophysiol 2007; 30(9):1096-1104.

18. Vardas PE, Auricchio A, Blanc JJ, Daubert JC, Drexler H, Ector H, Gasparini M, Linde C, Morgado FB, Oto A, Sutton R, Trusz-Gluza M. Guidelines for cardiac pacing and cardiac resynchronization therapy. The Task Force for Cardiac Pacing and Cardiac Resynchronization Therapy of the European Society of Cardiology. Developed in collaboration with the European Heart Rhythm Association. Europace 2007; 9(10):959-998.

19. Pina IL, Balady GJ, Hanson P, Labovitz AJ, Madonna DW, Myers J. Guidelines for clinical exercise testing laboratories. A statement for healthcare professionals from the Committee on Exercise and Cardiac Rehabilitation, American Heart Association. Circulation 1995; 91(3):912-921.

20. Rector TS, Kubo SH, Cohn JN. Patients' self-assessment of their congestive heart failure: content, reliability and validity of a new measure-the Minnesota Living with Heart Failure questionnaire. Heart Failure 1987; 3:198-209.

21. Schiller NB, Shah PM, Crawford M, DeMaria A, Devereux R, Feigenbaum H, Gutgesell H, Reichek $N$, Sahn D, Schnittger I, et al. Recommendations for quantitation of the left ventricle by twodimensional echocardiography. American Society of Echocardiography Committee on Standards, Subcommittee on Quantitation of Two-Dimensional Echocardiograms. J Am Soc Echocardiogr 1989; 2(5):358-367.

22. Thomas JD. How leaky is that mitral valve? Simplified Doppler methods to measure regurgitant orifice area. Circulation 1997; 95(3):548-550. 
23. Pitzalis MV, lacoviello M, Romito R, Massari F, Rizzon B, Luzzi G, Guida P, Andriani A, Mastropasqua F, Rizzon P. Cardiac resynchronization therapy tailored by echocardiographic evaluation of ventricular asynchrony. J Am Coll Cardiol 2002; 40(9):1615-1622.

24. Yu CM, Abraham WT, Bax J, Chung E, Fedewa M, Ghio S, Leclercq C, Leon AR, Merlino J, Nihoyannopoulos P, Notabartolo D, Sun JP, Tavazzi L. Predictors of response to cardiac resynchronization therapy (PROSPECT)—study design. Am Heart J 2005; 149(4):600-605.

25. Chung ES, Leon AR, Tavazzi L, Sun JP, Nihoyannopoulos P, Merlino J, Abraham WT, Ghio S, Leclercq C, Bax JJ, Yu CM, Gorcsan J, 3rd, St John Sutton M, De Sutter J, Murillo J. Results of the Predictors of Response to CRT (PROSPECT) trial. Circulation 2008; 117(20):2608-2616.

26. Stellbrink C, Breithardt OA, Franke A, Sack S, Bakker P, Auricchio A, Pochet T, Salo R, Kramer A, Spinelli J. Impact of cardiac resynchronization therapy using hemodynamically optimized pacing on left ventricular remodeling in patients with congestive heart failure and ventricular conduction disturbances. J Am Coll Cardiol 2001; 38(7):1957-1965.

27. Bleeker GB, Schalij MJ, Holman ER, Steendijk P, van der Wall EE, Bax JJ. Cardiac Resynchronization Therapy in Patients With Systolic Left Ventricular Dysfunction and Symptoms of Mild Heart Failure Secondary to Ischemic or Nonischemic Cardiomyopathy. Am J Cardiol 2006; 98(2):230-235.

28. Landolina M, Lunati M, Gasparini M, Santini M, Padeletti L, Achilli A, Bianchi S, Laurenzi F, Curnis A, Vincenti A, Valsecchi S, Denaro A. Comparison of the effects of cardiac resynchronization therapy in patients with class II versus class III and IV heart failure (from the InSync/InSync ICD Italian Registry). Am J Cardiol 2007; 100(6):1007-1012.

29. Linde C, Abraham WT, Gold MR, St John Sutton M, Ghio S, Daubert C. Randomized trial of cardiac resynchronization in mildly symptomatic heart failure patients and in asymptomatic patients with left ventricular dysfunction and previous heart failure symptoms. J Am Coll Cardiol 2008; 52(23):1834-1843.

30. Shimano M, Tsuji Y, Yoshida Y, Inden Y, Tsuboi N, Itoh T, Suzuki H, Muramatsu T, Okada T, Harata S, Yamada T, Hirayama H, Nattel S, Murohara T. Acute and chronic effects of cardiac resynchronization in patients developing heart failure with long-term pacemaker therapy for acquired complete atrioventricular block. Europace 2007; 9(10):869-874.

31. Delnoy PP, Ottervanger JP, Luttikhuis HO, Elvan A, Misier AR, Beukema WP, van Hemel NM. Long-term clinical response of cardiac resynchronization after chronic right ventricular pacing. Am J Cardiol 2009; 104(1):116-121. 
32. Epstein AE, DiMarco JP, Ellenbogen KA, Estes NA, 3rd, Freedman RA, Gettes LS, Gillinov AM, Gregoratos G, Hammill SC, Hayes DL, Hlatky MA, Newby LK, Page RL, Schoenfeld MH, Silka MJ, Stevenson LW, Sweeney MO, Smith SC, Jr., Jacobs AK, Adams CD, Anderson JL, Buller CE, Creager MA, Ettinger SM, Faxon DP, Halperin JL, Hiratzka LF, Hunt SA, Krumholz HM, Kushner FG, Lytle BW, Nishimura RA, Ornato JP, Page RL, Riegel B, Tarkington LG, Yancy CW. ACC/AHA/HRS 2008 Guidelines for Device-Based Therapy of Cardiac Rhythm Abnormalities: a report of the American College of Cardiology/ American Heart Association Task Force on Practice Guidelines (Writing Committee to Revise the ACC/AHA/NASPE 2002 Guideline Update for Implantation of Cardiac Pacemakers and Antiarrhythmia Devices) developed in collaboration with the American Association for Thoracic Surgery and Society of Thoracic Surgeons. J Am Coll Cardiol 2008; 51(21):e1-62. 
Irene E. van Geldorp 1,2,3

Tammo Delhaas ${ }^{4}$

Ben Hermans ${ }^{1}$

Kevin Vernooy ${ }^{1,2}$

Bernard Broers ${ }^{2}$

Julija Klimusina ${ }^{5}$

François Regoli ${ }^{5}$

Francesco F. Faletra ${ }^{5}$

Tiziano Moccetti ${ }^{5}$

Bart Gerritse ${ }^{6}$

Richard Cornelussen ${ }^{1,6}$

Jos J. Settels ${ }^{7}$

Harry J.G.M. Crijns ${ }^{2}$

Angelo Auricchio ${ }^{5}$

Frits W. Prinzen ${ }^{1}$

${ }^{1}$ Department of Physiology, Cardiovascular Research Institute Maastricht (CARIM), Maastricht University, Maastricht, The Netherlands; ${ }^{2}$ Department of Cardiology, Cardiovascular Research Institute Maastricht (CARIM), Maastricht University Medical Center, Maastricht, The Netherlands; ${ }^{3}$ Department of Pediatrics, Maastricht University Medical Center, Maastricht, The Netherlands; ${ }^{4}$ Department of Biomedical Engineering, Cardiovascular Research Institute Maastricht (CARIM), Maastricht University, Maastricht, The Netherlands; ${ }^{5}$ Division of Cardiology, Fondazione Cardiocentro Ticino, Lugano, Switzerland; ${ }^{6}$ Medtronic Bakken Research Center, Maastricht, The Netherlands; ${ }^{7}$ BMEYE B.V., Amsterdam, The Netherlands. 


\section{4}

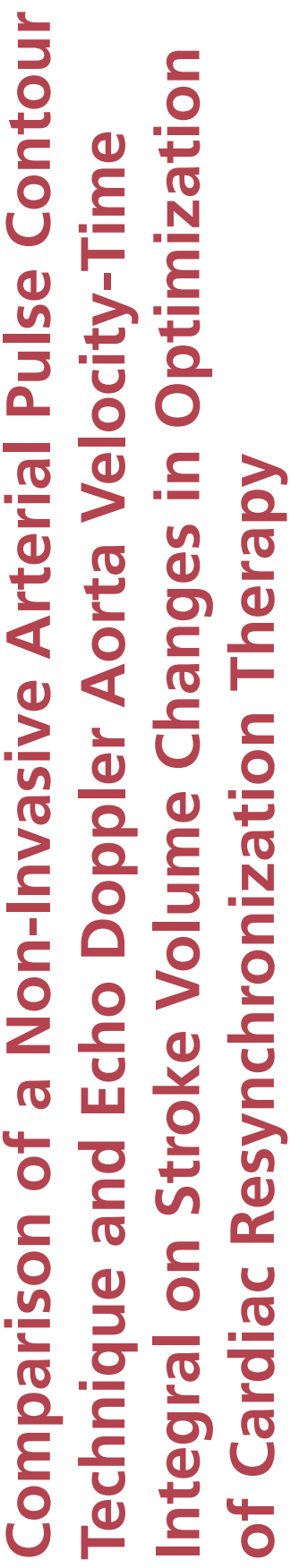




\section{Abstract}

Aims: We investigated the accuracy and feasibility of a non-invasive arterial pulse contour technique for continuous measurement of stroke volume (SV) in optimization of atrioventricular (AV) delay in cardiac resynchronization therapy (CRT), by comparing SV changes assessed by Nexfin CO-Trek ${ }^{\circledR}$ (Nexfin) and echo Doppler aortic velocity-time-integral (VTlao). Furthermore, we investigated whether AV-delay optimization increases the effect of CRT when compared with a default AV-delay (120ms).

Methods and results: In 23 CRT patients, biventricular pacing (BiVP) was applied at various AVdelays, while recording 10 beats preceding BiVP (baseline) and the first 10 BiVP beats, for both methods in parallel. Agreement between Nexfin and VTlao measurements was evaluated (BlandAltman) on beat-to-beat changes in SV, as well as on effects of BiVP (averaged over 8 beats) at various AV-delays. Individual optimal AV-delays, for Nexfin (AVopt-n) and VTlao (AVopt-ao), were derived from the second-order polynomial fitted to the effect measurements of 20 patients. In 252 episodes assessed, the difference between measurements (=Nexfin-VTlao) was $-0.6 \pm 8.1 \%$ for beat-to-beat SV-changes and $-1.3 \pm 7.3 \%$ for effects of BiVP. Optimal AV delays for Nexfin were well related to AVopt-ao $\left(R^{2}=0.69\right)$. The effect (\%) of BiVP at the optimal AV-delay was significantly larger than at the default AV-delay: median difference (range) being $+6.3 \%(0.1$ $14.4 \% ; p<0.001)$ for VTlao and $+4.7 \%(0.0-14.0 \% ; p<0.001)$ for Nexfin.

Conclusions: Individual AV-optimization increases the effect of CRT. Nexfin is a promising tool in individual CRT optimization, as Nexfin agrees with VTlao on measuring beat-to-beat SVchanges and on assessing relative effects of BiVP on SV at various AV-delays. 


\section{INTRODUCTION}

Cardiac resynchronization therapy (CRT) improves left ventricular (LV) pump function and reverses $L V$ remodelling through the restoration of synchrony in LV electrical activation in patients in a wide spectrum of mild to severe heart failure and prolonged QRS duration. The substantial evidence that CRT improves clinical status and that it reduces morbidity as well as mortality, has resulted in the incorporation of CRT in the management of dyssynchrony associated heart failure. Devices for CRT have become significantly more sophisticated, including the capability to independently program atrioventricular (AV) and interventricular (VV) stimulation timing intervals, which has been shown to acutely significantly influence cardiac function. ${ }^{1-4}$ Although tailoring the AV-delay and VV-interval to the individual patient could further increase the haemodynamic improvement and long term benefits brought by CRT, the best way to guide the optimization of these device settings is still under debate. Since CRT has been shown to acutely improve haemodynamic status, ${ }^{1}$ AV-delay and VV-interval might be optimized by haemodynamic monitoring. Gold standards for the measurement of haemodynamic status are the estimation of LV contractility by measuring the maximum rate of rise of LV pressure and the measurement of cardiac output by thermodilution or conductance catheter. These invasive methods, however, are not suitable for routine use in CRT optimization as they require cardiac catheterization, and thus impose significant biological and interventional risk to patient as well as add significant financial and human resource burden. Although echocardiographic assessment of the velocity-timeintegral of the aorta (VTIao) has been shown to be a good surrogate for stroke volume (SV),, 6 this measurement may be challenging in some patients due to limited or poor acoustic window. Furthermore, it is very time-consuming to obtain a reliable set of data over a series of heart beats for device optimization. Summarizing, there is great clinical need for a harmless, patient friendly and easy-to-use technique for (continuous) haemodynamic monitoring, suitable for being applied in individual optimization of CRT. A non-invasive method to continuously monitor haemodynamics, using a finger sensor, is provided by Nexfin ${ }^{\circledR}$ (BMEYE B.V., Amsterdam, Netherlands). We investigated whether Nexfin reliably measures beat-to-beat changes as well as immediate relative effects on SV induced by CRT at various AV-delays and, hence, whether the use of Nexfin is feasible in the individual optimization of CRT. The second aim was to investigate whether benefit of CRT is increased with AV-delay optimization when compared with a default AV-delay. 


\section{Methods}

\section{Subjects}

From the cardiology departments of Maastricht and Lugano, 23 outpatients (5 females; $22 \%$ ) were enrolled $2.9 \pm 2.6$ years after implantation of a biventricular pacemaker or biventricular defibrillator for refractory heart failure and left bundle branch block (LVEF $<35 \%$ and QRS $>130 \mathrm{~ms}$ ). Patient characteristics at implantation: QRS-duration 161 $\pm 25 \mathrm{~ms}$; LVEF 25 $\pm 7 \%$; median NYHA functional class 3 (range: $2-4$ ); heart failure aetiology: ischaemic $n=11$, nonischaemic $n=12$. Exclusion criteria for the current study were atrial fibrillation, non-optimal echocardiographic image-quality of the LV outflow tract which did not permit to assess VTlao (known from previous examinations), as well as the presence of moderate to severe aortic valve stenosis or regurgitation. At inclusion in the study, patient characteristics were: age $69 \pm 8$ years;

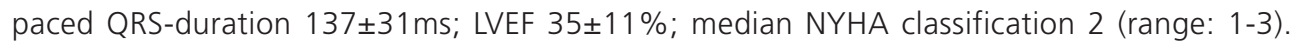
Patients gave informed consent for this study, which was approved by the institutional review boards of both centres.

\section{Study protocol}

In order to investigate the reliability of Nexfin CO-Trek in measuring SV changes, we compared relative SV changes assessed by Nexfin with relative changes in VTlao on the same beats. Stroke volume changes were induced by biventricular pacing (BiVP) at various AV-delays. The pacing protocol was initiated after the patient being in supine position for 15 minutes. To exclude haemodynamic variability induced by spontaneous changes in heart rate, and to increase the probability of a more pronounced peak in the immediate haemodynamic responses to CRT at the various AV-delays at higher heart rates, ${ }^{7}$ we enforced a constant heart rate by atrial overdrive pacing (90bpm). The protocol consisted of a baseline without ventricular pacing, alternated with atrial-based BiVP at various AV-delays in episodes of $\sim 30$ seconds (Figure 1). Starting at an AVdelay of $60 \mathrm{~ms}$ for the first BiVP intervention, the AV-delay was increased in $20 \mathrm{~ms}$ steps until either complete ventricular sensing occurred or until a maximum AV-delay of 340ms.

\section{Measurements and data acquisition}

During the entire protocol, arterial pressure and SV were continuously and non-invasively recorded by Nexfin. Nexfin uses an inflatable cuff that is placed around the mid-phalanx of the middle finger, which contains a built-in photo-electric plethysmograph. By a volume-clamp circuit combined with the Physiocal ${ }^{\circledR}$ method, $^{8,9}$ the finger arteries under the cuff are dynamically clamped to their unloaded volume, and the pressure used is determined and tracked over time, resulting in a dynamic and calibrated measurement of finger arterial pressure. The finger arterial pressure is automatically reconstructed into the brachial arterial pressure waveform. ${ }^{8-}$ 10 Stroke volume is automatically computed from the systolic area under the brachial arterial 


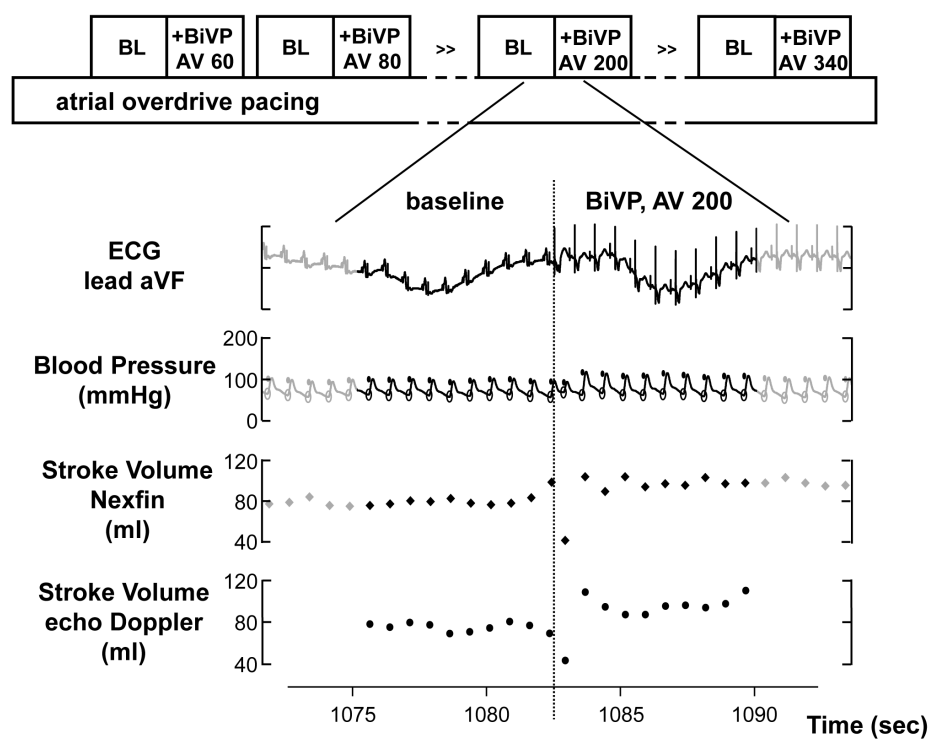

\section{Figure 1. Study design}

Along a baseline (BL) of constant heart rate enforced by atrial overdrive pacing without ventricular pacing, $S V$ changes were induced by episodes of biventricular pacing (BiVP) at various AV delays in the range of 60340ms (AV xx). Nexfin continuously recorded ECG and blood pressure, and computed beat-to-beat. In parallel, for each intervention echo Doppler investigation of the LV outflow tract was performed on at least the final 10 baseline beats and the first 10 BiVP beats. Off line the velocity-time-integral was manually estimated (for this example, SV was calculated from the velocity-time-integral and cross sectional area of the aorta).

pressure pulse contour. Patient's age, gender, height, and weight are important input parameters for the patient individualization of the SV measurement using the Nexfin CO-Trek ${ }^{\circledR}$ algorithm. ${ }^{10}$ Simultaneously, ECG signal was recorded with the ECG module of Nexfin. Offline, data on SV as measured by Nexfin from the last 10 individual heartbeats preceding BiVP (baseline beats) and the first 10 beats during BiVP (registration in black, Figure 1) were selected and exported using customized software based on the Matlab platform (MathWorks, Natick, MA, USA) after conversion by Framelnspector (version 1.22.0.0, BMEYE) to a format compatible with Matlab (.bin). In parallel with the Nexfin recordings, continuous wave Doppler echocardiography (Vivid 7, General Electric, Milwaukee, WI, USA) of the aorta was performed on the same beats (10 baseline beats + 10 BiVP beats). The echo Doppler recordings were stored and the VTlao was manually measured offline using EchoPAC post-processing software (Dimension BT08, General Electric). Stroke volume can directly be derived from the VTlao, by multiplying the VTlao with the aortic 
cross sectional area. Though, since this study focuses on the comparison of relative changes in SV, we did not calculate absolute SV for echo measurements.

\section{Statistical methods}

\section{Comparison of Nexfin and echo Doppler: on the assessment of beat-to-beat stroke volume changes and on the estimation of the effect on stroke volume as induced by biventricular pacing}

To allow pooling of the data of the various episodes and different patients, the mean values of each of the baselines, for both Nexfin and VTlao, served as a reference ( $0 \%$ level in Figure 2, upper panel). Then, the relative deviation (RD, \%) from the reference of the episode was calculated for each of the 20 beats in that episode, for both Nexfin and VTlao (RDSVn and RDSVao, respectively; Figure 2, upper panel). The first objective was to identify the agreement of Nexfin and echo Doppler on the measurement of beat-to-beat SV changes due to either spontaneous variability or artificially induced variability by BiVP. For this purpose, the differences between the measurements of Nexfin and VTlao on relative beat-to-beat SV changes were calculated (Figure 2, lower panel) and evaluated according to the suggested method of analysis by Bland and Altman. ${ }^{11}$ In this method, the mean difference between the methods is defined as 'bias'. The upper and lower limits of agreement are defined as 'bias $\pm 1.96 \times$ SD of the difference between the methods'. The second step in the comparison of Nexfin and echo Doppler was to investigate the agreement of the methods on the assessment of the effect on SV as induced by BiVP at the various AV-delays. Because SV is strongly affected by changes in RR-intervals due to either lengthening or shortening of the AV-delay (see also the example given in Figure 1), premature and post-premature beats, as well as the first and the second beats after transition were excluded. Subsequently, we assessed the relative effect of BiVP on SV for each AV-delay by calculating the mean relative SV increase or decrease over the BiVP beats (= mean RDSVao and RDSVn over BiVP beats) and defined these as 'effect-SVao' and 'effect-SVn', for echo Doppler VTlao and Nexfin, respectively (Figure 2, upper panel). The Bland-Altman analysis was performed on effect-SVao and effect-SVn, to identify the agreement between Nexfin and echo Doppler on the assessment of the relative effects on SV induced by BiVP at various AV-delays.

\section{Atrioventricular-optimization curves for Nexfin and echo Doppler}

Individual optimization curves for echo Doppler and Nexfin were obtained by a second-order polynomial curve fitted to the data on the relative effects, effect-SVao and effect-SVn, respectively (Figure 4). The AV-delay at the location of the maximal effect (peak of the curve) was defined as the optimal AV-delay (AVopt-ao and AVopt-n, for echo Doppler and Nexfin respectively). The difference in effect-SVao at AVopt-n and at AVopt-ao, was used as a measure of the haemodynamic relevance of the discrepancy between AVopt-n and AVopt-ao. For both echo Doppler and Nexfin, the range of optimal AV-delays was defined as the part of the curve 
where the relative effect was less than $2 \%$-points lower than the effect at the optimal AV-delay. Lastly, the maximal relative effect was compared with the relative effect at a default AV-delay of 120 ms to investigate whether individual AV-delay optimization is beneficial.

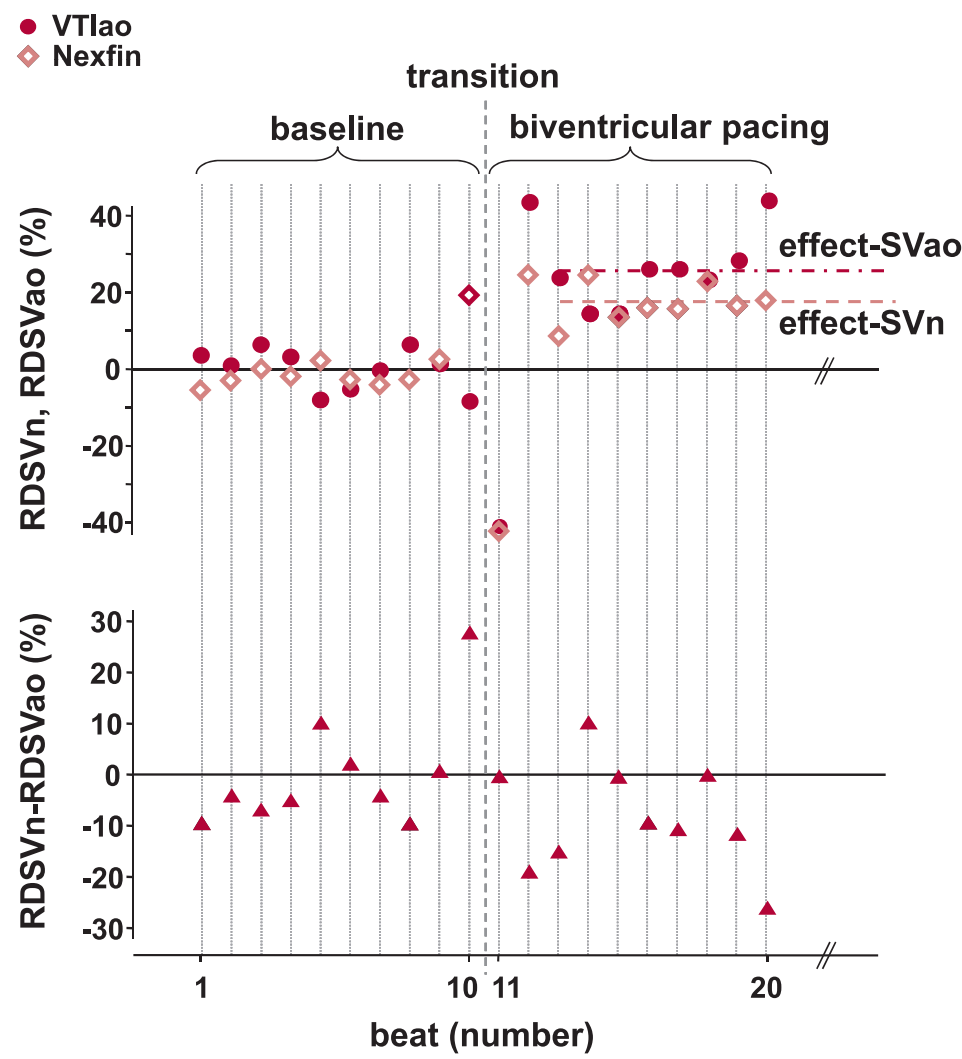

Figure 2. Statistical design

In each episode, the relative deviation from the mean of the baseline beats was calculated for stroke volume measured by Nexfin as well as for stroke volume (SV) derived from echo Doppler investigation (RDSVn and RDSVao, respectively) for each of the 20 beats in that episode (upper panel). For each AV delay we assessed the relative effect of biventricular pacing (BiVP) on SV, by calculating the mean relative SV increase or decrease over the BiVP beats (= mean RDSVao and RDSVn over BiVP beats, excluding the first and second beats after transition) and defined these as 'effect SVao' and 'effect SVn', for echo Doppler velocity-time-integral (VTIao) and Nexfin, respectively (upper panel). The differences between RDSVn and RDSVao (lower panel), as well as the differences between the effect-measurements were calculated for subsequent analysis on the agreement of Nexfin and echo Doppler. 


\section{RESULTS}

\section{Protocol complications}

In 23 patients, a total of 252 BiVP episodes at various AV-delays were assessed with an average of $11 \pm 3$ interventions for each individual patient. In one patient, from an AV-delay of $200 \mathrm{~ms}$ onwards, the device (St. Jude Medical Systems; Promote RF 3213-36) could not be programmed in 20ms steps, therefore the AV-delays tested were subsequently 225, 250, 275, 300 and $350 \mathrm{~ms}$. In three patients the protocol was performed using overdrive pacing at a rate of $75 \mathrm{bpm}$ $(n=1)$ or $80 \mathrm{bpm}(n=2)$, since progressive AV-conduction disturbances occurred at higher rates.

\section{Agreement between Nexfin and echo Doppler; on the assessment of relative beat-to-beat stroke volume changes and on the estimation of the effect on stroke volume as induced by biventricular pacing}

For a total of 5028 beats, measurements by both Nexfin and echo Doppler were available. From the Bland-Altman analysis on the beat-to-beat relative deviations (step1), the observed variance (SD) on the measurement differences between the methods (=RDSVn-RDSVao) was $\pm 8.1 \%$ around a mean bias of $-0.6 \%$ (limits of agreement: $\pm 15.9 \%$ ). The inter-beat variability on baseline beats ( $n=2422$ after exclusion of premature and post-premature beats), was $\pm 4.7 \%$ for RDSVn and $\pm 6.3 \%$ for RDSVao. The second step in the comparison of Nexfin with echo Doppler was the use of the Bland-Altman analysis for the evaluation of the agreement between the methods on the assessment of the relative effect of BiVP on SV when compared with baseline ('effect-SVao' and 'effect-SVn', as visualized in Figure 3). By means of the Bland-Altman analysis, the difference between Nexfin and echo Doppler (effect-SVn-effect-SVao) was $-1.3 \pm 7.3 \%$ (limits of agreement: $\pm 14.3 \%$ ). The variances (SD) observed on the measurement differences between the methods, as well as the limits of agreement (bias $\pm 1.96 \times \mathrm{SD}$ ), are indicative for a good agreement between the methods and suggest that Nexfin CO-Trek is reliable in the measurement of SV changes (the reliability of Nexfin is further exemplified in the 'Discussion' section).

\section{Atrioventricular-optimization curves for Nexfin and echo Doppler}

In the third step of the comparison of Nexfin and echo Doppler in CRT optimization, optimization curves for both Nexfin and echo Doppler could be obtained for 20 individual patients. (One patient was excluded for this analysis, because the occurrence of multiple premature beats in each episode implicated that the measurements on relative effects were not representative and thus not useful for optimization. In two patients optimization curves couldn't be fitted to the individual data because of a large 'back and forth variation' between the settings). Regarding the shape of the curves and their fit to the individual effect measurements, variation between patients was observed. Figure 4 demonstrates four different explicative examples, all with a good agreement 

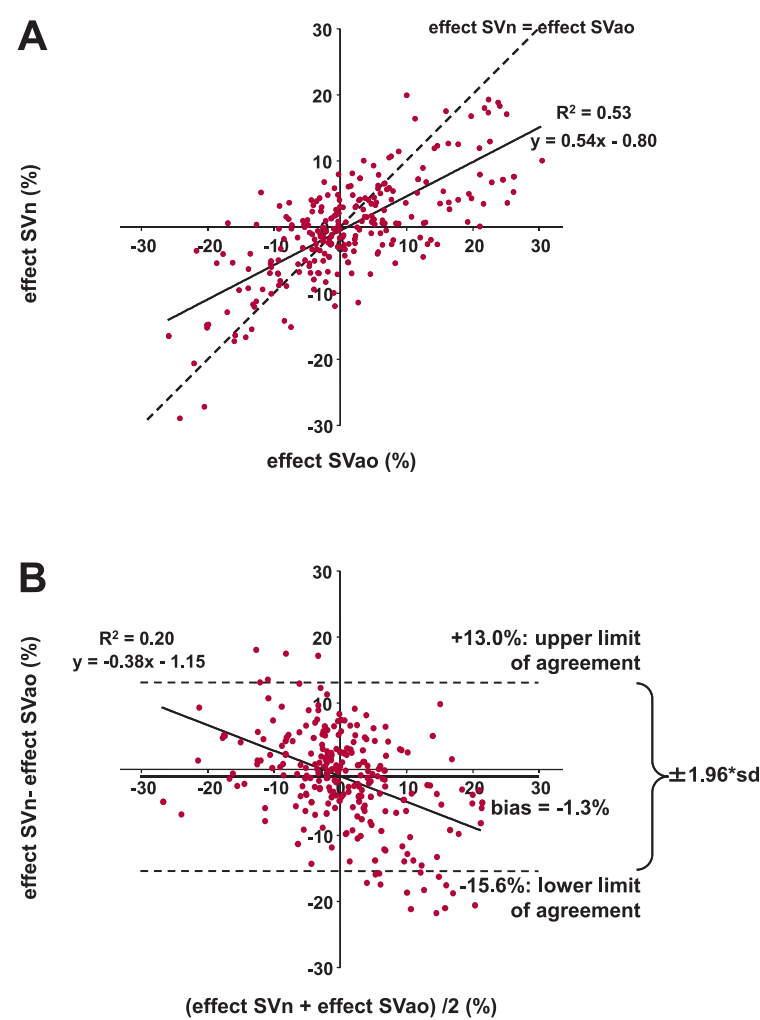

Figure 3. Agreement between Nexfin and echo Doppler on the assessment of the relative effect on stroke volume as induced by biventricular pacing

The relative effect on stroke volume induced by biventricular pacing at various AV delays, as measured by Nexfin (effect $S V n$ ) is plotted against the relative effect measured with the use of echo Doppler (effect SVao) (A). The difference between effect SVn and effect SVao is displayed against the average of both measurements (B).

between Nexfin and echo Doppler on the individual effect values (limits of agreement for the individual patients are respectively, $A: \pm 17.6 \% ; B: \pm 11.0 \% ; C: \pm 13.9 \%$; and $D: \pm 11.8 \%)$. The optimization curves in panel A show a positive effect of BiVP for all AV-delays tested. The curves well fit the individual effect-values (echo: $R^{2}=0.93$ and Nexfin: $R^{2}=0.80$ ) and AVopt-n (258ms) is close to AVopt-ao (270ms). In the example shown in panel $B$, the optimization curve for echo Doppler fits the individual points only moderately $\left(R^{2}=0.51\right)$. Nevertheless, AVopt-ao (260ms) and AVopt-n (257ms) are almost similar. In the patient presented in panel $\mathrm{C}$ optimization curves have a good fit to the individual data points (echo: $R^{2}=0.84$ and Nexfin: $R^{2}=0.82$ ), but there is less 
agreement between Nexfin and echo Doppler on the optimal AV-delay $(186 \mathrm{~ms}$ and $260 \mathrm{~ms}$ for AVopt-n and AVopt-ao, respectively). In the patient presented in panel $D$, the accordances of the curves with the individual data and consensus of the optimal AV-delay derived from Nexfin and echo Doppler are poor ( $281 \mathrm{~ms}$ and $211 \mathrm{~ms}$ for AVopt-n and AVopt-ao, respectively). On average, the correlation of the fitted optimization curves and the individual effect-data was similar for Nexfin $\left(R^{2}=0.63 \pm 0.24\right)$ and echo Doppler $\left(R^{2}=0.68 \pm 0.27\right)$. The averaged maximum effect as derived from the Nexfin optimization curves was $4.3 \pm 5.4 \%$, which was significantly smaller than the maximal effect as derived from the echo curves $(7.2 \pm 7.7 \% ; p=0.008)$. Optimal AV delay for Nexfin and AVopt-ao were well correlated, as is shown in Figure 5 (Patients A-D are corresponding to the patients in Figure 4). The absolute difference in AV-delay (ms) between AVopt-n and AVopt-ao was $\leq 20$ ms for 13 out of 20 patients ( $65 \%$ ), indicating (almost) similarity of AVopt-n and AVopt-ao in these patients (Figure 5). In the majority of the patients (80\%; $\mathrm{n}=16$ ), the difference in relative effect by BiVP as measured with echo at the AVopt-ao and at AVopt-n was $<1 \%$-points (difference in the effect of total population: median $0.2 \%$-points; range 0.0-5.2\%-points). This might be clinically even more important than an exact similarity of AVopt-n and AVopt-ao, as the small difference in effect indicates just only a minimal
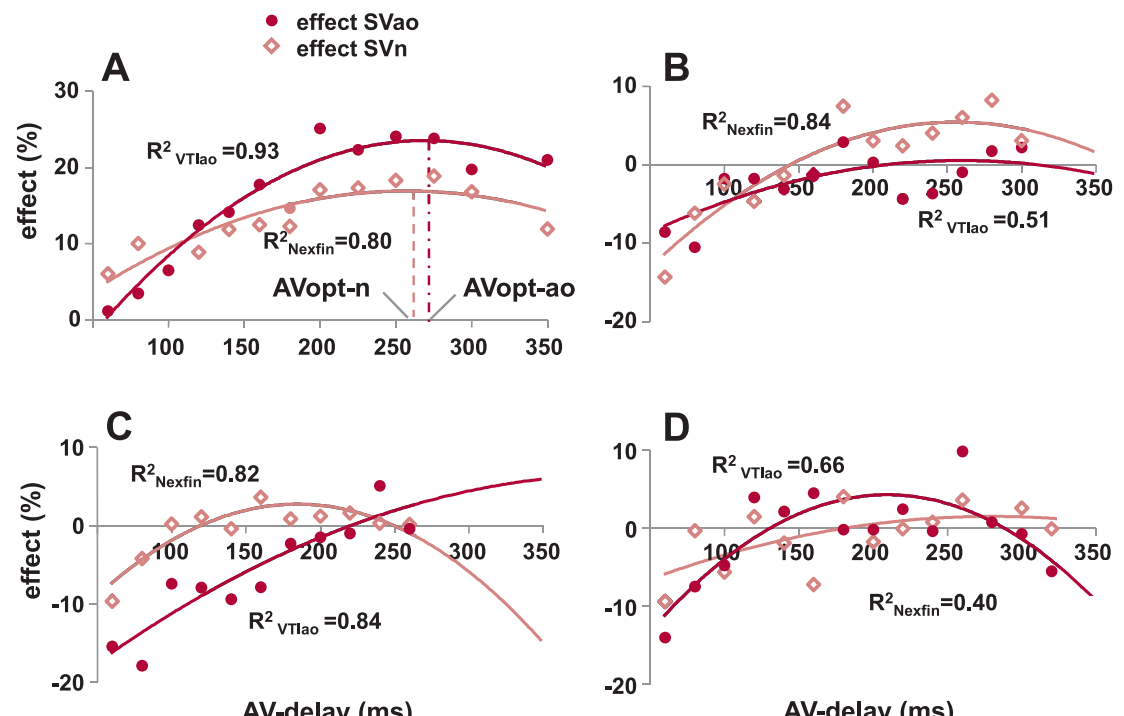

Figure 4. Individual curves for AV optimization

$A, B, C$ and $D$ are four different explicative examples of individual optimization curves as derived from the measurements of relative effect on stroke volume induced by biventricular pacing at various AV delays, as assessed by Nexfin and echo Doppler. The AV delay corresponding to the maximal relative effect (top of the curve) was defined as the optimal AV delay (AVopt-ao and AVopt-n, for echo Doppler and Nexfin, respectively). 
haemodynamic relevance of the discrepancy between AVopt-n and AVopt-ao. For both echo Doppler and Nexfin, a range of optimal AV-delays was defined as the part of the curve where the relative effect was $<2 \%$-points lower than the effect (\%) at the optimal AV-delay. The width of this range of optimal AV-delays was $110 \pm 34 \mathrm{~ms}$ for Nexfin and $97 \pm 47 \mathrm{~ms}$ for echo, suggesting that there may be a broad range of AV-delays leading to (almost) optimal effects. Furthermore, there was a great similarity for Nexfin and echo Doppler in this optimal range, as indicated by $73 \pm 21 \%$ overlap of the Nexfin optimal range with the optimal range of echo Doppler. The relative effect as achieved by optimizing the AV-delay was significantly larger than the effect using a default ("out-of-the-box") AV-delay of $120 \mathrm{~ms}$ : $7.2 \pm 7.7 \%$ versus $0.6 \pm 9.4 \%$ (median difference in effect $+6.3 \%$; range $0.1-14.4 \%$; $p<0.001$ ) as estimated by echo Doppler and $4.3 \pm 5.4 \%$ versus $-0.6 \pm 7.2 \%$ (median difference $+4.7 \%$; range $0.0-14.0 \% ; p<0.001$ ) by Nexfin.

\section{Discussion}

In the current study, we investigated the accuracy and feasibility of continuous non-invasive SV measurements by Nexfin CO-Trek in CRT optimization, by comparing Nexfin with echo Doppler at three levels: the measurement of beat-to-beat changes in $\mathrm{SV}$, the assessment of relative effects

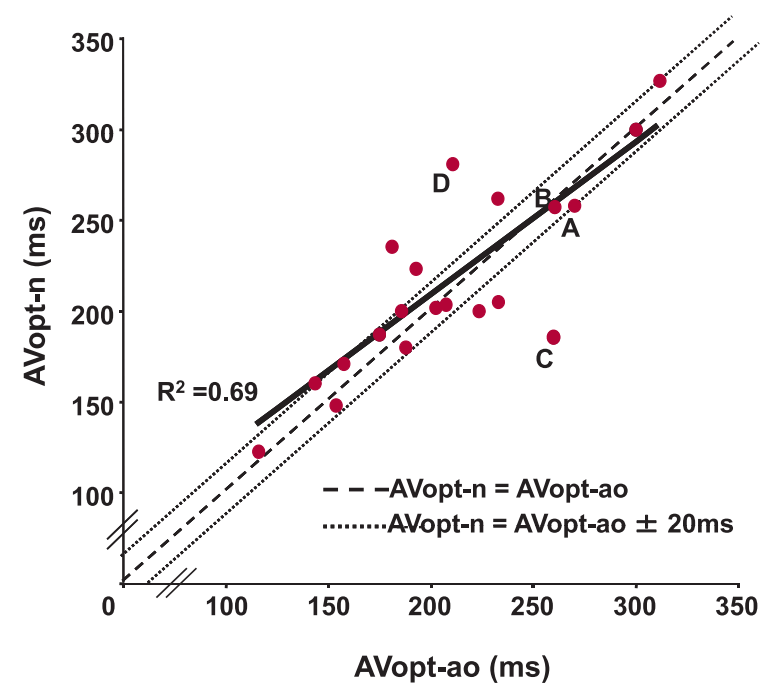

Figure 5. Correlation of individual optimal AV delays assessed using Nexfin and optimal AVdelays assessed using echo Doppler

AVopt-n: optimal atrioventricular (AV) delay as defined with the use of Nexfin; AVopt-ao: optimal AV delay as assessed with echo Doppler. (The indices for the individual cases A, B, C, and D correspond to the selected patients for figure 4). 
of BiVP at various AV-delays, and the determination of the optimal AV-delay. The most important findings are that Nexfin has a good agreement with VTlao by echo Doppler on the measurement of changes in SV due to either physiological beat-to-beat alterations or to changes induced by BiVP, and that in individual optimization both methods Nexfin and VTlao determine similar AVdelays as the optimal AV-delay. Additionally, our data suggest that individual optimization of the AV-delay leads to better haemodynamic improvement of CRT than programming a default AVdelay of $120 \mathrm{~ms}$.

\section{Reliability of Nexfin in the assessment of acute haemodynamic changes}

To investigate the reliability of Nexfin CO-Trek on the assessment of acute haemodynamic changes, we used the VTlao assessed by echo Doppler to compare Nexfin CO-Trek with, as this method provides a validated non-invasive measurement of (changes in) SV. ${ }^{5,6}$ Optimizing the VTlao is also one of the recognized and accurate methods for CRT optimization. ${ }^{12}$ The variance $( \pm$ SD) on the measurement differences between Nexfin and echo Doppler, was $\pm 8.1 \%$ for the measurement of beat-to-beat changes. This observed variance can be entirely explained by the summation of the variability in each of the methods, since by analysing exactly the same beats for both methods, physiological variability is excluded as a confounding factor. The measurement variability for each method separately can be hypothesized from the equation for combined variances [combined variance $=\sqrt{ }\left(\right.$ variance $A^{2}+$ variance $\left.\left.B{ }^{2}\right)\right] .{ }^{13}$ If the measurement variabilities on SV changes of echo Doppler and Nexfin are assumed to be equal, these variabilities would be $5.7 \%\left[\sqrt{ }\left(5.7^{2}+5.7^{2}\right)=8.1\right]$. If the measurement variability of echo Doppler is assumed to be higher, the precision of Nexfin would even be better: for the theoretical example that the measurement variability for echo Doppler is $7.0 \%$, the variability of Nexfin would be $4.1 \%$ $\left[\sqrt{ }\left(7.0^{2}+4.1^{2}\right)=8.1\right]$. When compared with the available haemodynamic measurements, a method with a $~ 5 \%$ inconsistency in the assessment of beat-to-beat SV changes may be considered to be very precise. ${ }^{14-16}$ In clinical practice, haemodynamic measurements are usually performed on multiple beats. Therefore, it seems more appropriate to judge the reliability of Nexfin based on the variance on the difference between the effect-measurements of Nexfin and echo Doppler (derived from averages of multiple beats), which was the second step in our comparison. The variance on these measurement differences was $\pm 7.3 \%$. Consequently, if the measurement variability on the assessment of the effect on SV by BiVP are assumed to be equal for echo Doppler and Nexfin, these variabilities would be $5.2 \%\left[\sqrt{ }\left(5.2^{2}+5.2^{2}\right)=7.3\right]$.

From Figure $3 A$ and $B$ (trendline), as well as from the finding that the optimal effect was smaller when measured with Nexfin (4.3 $\pm 5.4 \%)$ when compared with echo Doppler $(7.2 \pm 7.7 \%)$, it can be deduced that using Nexfin, changes in SV are estimated to be smaller than that using echo Doppler. This may also explain the smaller inter-beat variance on baseline beats for Nexfin $( \pm 4.7 \%)$ when compared with echo Doppler $( \pm 6.3 \%)$. These findings appear to be in keeping 
with observations of Butter et al. ${ }^{17}$ that finger blood pressure measurements underestimate haemodynamic changes, but also that these measurements provide highly specific predictions of positive and negative changes in aortic pulse pressure. Moreover, the reliability of an effect measurement is influenced by the size of the effect and the standard error of the mean effect (SEM). In the current study, the SEM can be derived from the inter-beat variance (combination of physiological inter-beat variability and measurement error on each beat), divided by the square root of the number of beats on which the effect is determined $(n=8)$. The ratio between the effect and SEM may be used as a measure for reliability of the effect measured [averaged optimal effect / SEM]. For the averaged optimal effect on SV by BiVP this ratio was $2.6[4.3 \% /(4.7 \% / \sqrt{ } 8)$ $=2.6]$ for Nexfin, and even a little better for echo Doppler [7.2\% / $(6.3 \% / \sqrt{ } 8)=3.2]$. However, it should be kept in mind that for the effect measurements in the present study, VTlao tracing was performed using careful off-line analysis of 8 paced heart beats (in comparison with 10 baseline beats), whereas in clinical practice, often only 1-3 beats are used. By virtue of this lower number SEM will increase and reliability will therefore decrease, and will decrease even more if the beats are not randomly spread over the respiratory cycle.

\section{Defining the optimal atrioventricular-delay using individual optimization curves}

There was no clinically relevant difference between the AV-delays corresponding to the peaks of the individual optimization curves of Nexfin and echo Doppler, since there was a good correlation between the optimal AV-delays and a great overlap of the ranges of optimal AVdelays (where the effect of BiVP was not relevantly different from the effect of BiVP at the optimal AV-delay). These findings suggest that Nexfin reliably defines the best AV-delay setting in accordance with echo Doppler VTlao. These optimal AV-delays were identified from individual optimization curves for echo Doppler and Nexfin as obtained by a second order polynomial fitted to the data on effect-SVao and effect-SVn (Figure 4). Whinnett et al. demonstrated that the curve of response to BiVP at various AV-delays fits closely to a second order polynomial. ${ }^{3}$ In our study the fit of the optimization curves with the data was not as good as in the study by Whinnett et al., as reflected by lower mean R-values for both Nexfin and echo Doppler. This discrepancy may be explained by the inclusion of shorter AV-delays (40ms) by Whinnett, resulting in extreme worsening of haemodynamics, and therefore improving the correlation coefficient. An additional explanation may be that Whinnett et al. used repeated measurements on the effect of BiVP for each AV-setting. This was not practical in our study, because this was not the first aim of our study and would have increased the duration of the patient protocol significantly, due to the time consuming echo Doppler measurements. If only Nexfin would be used, measurements can be performed repeatedly in order to achieve more accurate optimization curves. 


\section{Clinical implications}

Cardiac resynchronization therapy is shown to be beneficial and is broadly applied in heart failure patients, though up to $30 \%$ of the patients are not fully responding to CRT. Some of the underresponders may turn into responders by better application of CRT. Despite proven acute haemodynamic benefits of individual optimization of AV-delay and VV-intervals, ${ }^{2,3}$ it is not yet proven whether or not CRT optimization significantly reduces the number of non-responders or has important impact on patient prognosis. Interestingly, preliminary results of the CLEAR study report that AV-delay optimization using an accelerometer substantially increases the number of patients responding to CRT, and reduces the number of major clinical events including hospitalization for heart failure and death. ${ }^{18,19}$ The benefit of individual optimization of the AVdelay is also indicated by our data as the optimal AV-delay varied between patients and as the relative effect on SV was significantly better at the optimal AV-delay than at a default AV-delay of $120 \mathrm{~ms}$. Long term effects of CRT cannot be directly predicted from the immediate haemodynamic effects achieved in optimization procedures. Nevertheless, improvements in haemodynamics have been shown to persist over time ${ }^{20}$ and have been associated with diminished energy costs, ${ }^{21}$ indicating improvement of mechanical efficiency which may last on the long term and may increase cardiac reserve. ${ }^{22}$ Additionally, in a recent study using invasive pulse-contour measurements, ${ }^{23}$ patients in whom an acute $\mathrm{SV}$ increase of $>5 \%$ by optimized CRT was achieved, showed a better clinical as well as echocardiographic response at 6-8 weeks follow-up, when compared with patients in whom $<5 \%$ acute SV increase was achieved. Nexfin immediately and automatically computes SV and, as showed in our study, is reliable in the assessment of SV changes. In contrast to other haemodynamic measurement techniques in CRT optimization procedures, Nexfin is non-invasive, patient friendly, and does not require great skill. As the main purpose of this study was to evaluate the reliability of Nexfin in the optimization of CRT and, hence, a steady and high heart rate was preferred, ${ }^{7}$ we performed (overdrive)pacing. In clinical practice, however, it would be important to also determine the optimal AV-delay for atrial sensed ventricular pacing, as this may better reflect physiological circumstances. Moreover, it would be interesting to optimize even at various heart rates and during exercise. ${ }^{24,25}$ One of the major advantages of using Nexfin above using echocardiography is that it can easily be applied in different body positions and even during exercise. Another advantage of the simple application of Nexfin is that optimization procedures can easily be repeated multiple times over longer time course, as it is likely that on the long term and/or in real life the optimal CRT settings will change. ${ }^{26}$

Prospective randomized studies are needed to clarify the real clinical impact of CRT optimization on the patients course. In this regard, the method for optimization may be of critical importance. Echocardiography is the most common approach to optimize AV- and VVtiming, ${ }^{27}$ yet it is a time-consuming approach and uses significant human resources. Thus, there is the quest for an alternative, inexpensive and highly reliable method which can be included as 
part of routine device follow-up. With the easy and relatively inexpensive use of Nexfin, all patients could undergo individual optimization.

\section{Study limitations}

The Bland-Altman evaluation of agreement assumes independent observations. ${ }^{28}$ Contrary to this assumption, we used multiple measurements on SV within each subject, where the true value varies due to (spontaneous or artificially induced) changes in haemodynamics. Variances on the biases and intervals within the limits of agreement were observed between the subjects (mean interval within the limits of agreement: $15.6 \pm 5.5 \%$; mean bias $-0.6 \pm 2.6 \%$ ) which may implicate that the interval between the observed limits of agreement for the total study group might be narrower than it should be, but probably only slightly. ${ }^{28}$ Measurements on the effect of BiVP were performed once for each AV-delay, since repetition was not feasible in our protocol with the parallel echocardiographic data acquisition. Nevertheless, for each AV-delay effectmeasurements should be performed repeatedly to define the individual optimal AV-delay more reliably. In the current study it was practically not feasible to perform both AV- and VV optimization (due to the parallel assessment of SV changes by Nexfin and VTlao). Because the results of our study indicate that Nexfin is reliable in the assessment of SV changes, we feel that parallel assessment of VTlao, next to Nexfin, is not longer necessary in subsequent studies.

\section{CONCLUSIONS}

Individual optimization of the AV-delay may increase the beneficial response to CRT. Nexfin COTrek provides an easy, non-invasive and patient friendly method to evaluate changes in SV due to either beat-to-beat alterations or to different settings of AV-delays. As Nexfin has a good precision on the measurement of beat-to-beat SV changes, the assessment of relative effects induced by BiVP and on the determination of the optimal AV-delay, we judge Nexfin as a promising tool in the individual optimization of CRT. 


\section{Funding}

This study was supported in part by an unrestricted grant from BMEYE B.V. (Amsterdam, The Netherlands).

\section{Disclosures}

A.Auricchio is consultant for Medtronic and Sorin and EBR Sytems; he receives speaker fee from Medtronic, Biotronik, Sorin and St.Jude Medical; his institution participates in industry-sponsored trials from Medtronic, Biotronik, Sorin, Boston Scientific, EBR Systems and St Jude Medical. F.W.Prinzen is consultant to Medtronic and has received research grants from Medtronic, Boston Scientific and EBR Systems. R.Cornelussen and B.Gerritse are employees of Medtronic. J.J.Settels is employee of BMEYE B.V.. 


\section{REFERENCES}

1. Auricchio A, Stellbrink C, Block M, Sack S, Vogt J, Bakker P, Klein H, Kramer A, Ding J, Salo R, Tockman B, Pochet T, Spinelli J. Effect of pacing chamber and atrioventricular delay on acute systolic function of paced patients with congestive heart failure. The Pacing Therapies for Congestive Heart Failure Study Group. The Guidant Congestive Heart Failure Research Group. Circulation 1999; 99(23):2993-3001.

2. Auricchio A, Stellbrink C, Sack S, Block M, Vogt J, Bakker P, Huth C, Schondube F, Wolfhard U, Bocker D, Krahnefeld O, Kirkels $\mathrm{H}$. Long-term clinical effect of hemodynamically optimized cardiac resynchronization therapy in patients with heart failure and ventricular conduction delay. J Am Coll Cardiol 2002; 39(12):2026-2033.

3. Whinnett ZI, Davies JE, Willson K, Manisty CH, Chow AW, Foale RA, Davies DW, Hughes AD, Mayet J, Francis DP. Haemodynamic effects of changes in atrioventricular and interventricular delay in cardiac resynchronisation therapy show a consistent pattern: analysis of shape, magnitude and relative importance of atrioventricular and interventricular delay. Heart 2006; 92(11):1628-1634.

4. Perego GB, Chianca R, Facchini M, Frattola A, Balla E, Zucchi S, Cavaglia S, Vicini I, Negretto M, Osculati G. Simultaneous vs. sequential biventricular pacing in dilated cardiomyopathy: an acute hemodynamic study. Eur J Heart Fail 2003; 5(3):305-313.

5. Bouchard A, Blumlein S, Schiller NB, Schlitt S, Byrd BF, 3rd, Ports T, Chatterjee K. Measurement of left ventricular stroke volume using continuous wave Doppler echocardiography of the ascending aorta and M-mode echocardiography of the aortic valve. J Am Coll Cardiol 1987; 9(1):75-83.

6. Gola A, Pozzoli M, Capomolla S, Traversi E, Sanarico M, Cobelli F, Tavazzi L. Comparison of Doppler echocardiography with thermodilution for assessing cardiac output in advanced congestive heart failure. Am J Cardiol 1996; 78(6):708-712.

7. Whinnett ZI, Davies JE, Willson K, Chow AW, Foale RA, Davies DW, Hughes AD, Francis DP, Mayet J. Determination of optimal atrioventricular delay for cardiac resynchronization therapy using acute non-invasive blood pressure. Europace 2006; 8(5):358-366.

8. Eeftinck Schattenkerk DW, van Lieshout JJ, van den Meiracker AH, Wesseling KR, Blanc S, Wieling W, van Montfrans GA, Settels JJ, Wesseling KH, Westerhof BE. Nexfin noninvasive continuous blood pressure validated against Riva-Rocci/Korotkoff. Am J Hypertens 2009; 22(4):378-383.

9. Harms MP, Wesseling KH, Pott F, Jenstrup M, Van Goudoever J, Secher NH, Van Lieshout JJ. Continuous stroke volume monitoring by modelling flow from non-invasive measurement of arterial pressure in humans under orthostatic stress. Clin Sci (Lond) 1999; 97(3):291-301.

10. Perel A, Settels JJ. The Nexfin HD monitor. Encyclopedia of Intensive Care Medicine. Berlin: Springer Verlag; 2010.

11. Bland JM, Altman DG. Statistical methods for assessing agreement between two methods of clinical measurement. Lancet 1986; 1(8476):307-310. 


\section{Chapter 4}

12. Thomas DE, Yousef ZR, Fraser AG. A critical comparison of echocardiographic measurements used for optimizing cardiac resynchronization therapy: stroke distance is best. Eur J Heart Fail 2009; 11(8):779-788.

13. Critchley LA, Critchley JA. A meta-analysis of studies using bias and precision statistics to compare cardiac output measurement techniques. J Clin Monit Comput 1999; 15(2):85-91.

14. de Wilde RB, Geerts BF, Cui J, van den Berg PC, Jansen JR. Performance of three minimally invasive cardiac output monitoring systems. Anaesthesia 2009; 64(7):762-769.

15. de Wilde RB, Schreuder JJ, van den Berg PC, Jansen JR. An evaluation of cardiac output by five arterial pulse contour techniques during cardiac surgery. Anaesthesia 2007; 62(8):760-768.

16. van Lieshout JJ, Toska K, van Lieshout EJ, Eriksen M, Walloe L, Wesseling KH. Beat-to-beat noninvasive stroke volume from arterial pressure and Doppler ultrasound. Eur J Appl Physiol 2003; 90(1-2):131-137.

17. Butter C, Stellbrink C, Belalcazar A, Villalta D, Schlegl M, Sinha A, Cuesta F, Reister C. Cardiac resynchronization therapy optimization by finger plethysmography. Heart Rhythm 2004; 1(5):568575 .

18. Delnoy PP, Lunati M, Nägele H, Padeletti L, Silvestre J, Martino M, Ritter P. Periodic VV and AV delays optimization in cardiac resynchronization therapy improves patients' clinical outcome: results from the CLEAR study. Heart Rhythm 2010; 7(5;S1):S55.

19. Padeletti L, Szwed H, Zupan I, Hartog H, Delarche N, Martino M, Ritter P. Clinical efficacy of CRT continuous optimization with SONR versus standard clinical practice. Heart Rhythm 2010; 7(5;S1):S56.

20. Vernooy K, Cornelussen RNM, Verbeek XAAM, Vanagt WYR, van Hunnik A, Kuiper M, Arts T, Crijns HJGM, Prinzen FW. Cardiac resynchronization therapy cures dyssynchronopathy in canine left bundle-branch block hearts. Eur Heart J 2007; 28(17):2148-2155.

21. Nelson GS, Berger RD, Fetics BJ, Talbot M, Spinelli JC, Hare JM, Kass DA. Left ventricular or biventricular pacing improves cardiac function at diminished energy cost in patients with dilated cardiomyopathy and left bundle-branch block. Circulation 2000; 102(25):3053-3059.

22. Steendijk P, Tulner SA, Bax JJ, Oemrawsingh PV, Bleeker GB, van Erven L, Putter H, Verwey HF, van der Wall EE, Schalij MJ. Hemodynamic effects of long-term cardiac resynchronization therapy: analysis by pressure-volume loops. Circulation 2006; 113(10):1295-1304.

23. Dizon JM, Quinn TA, Cabreriza SE, Wang D, Spotnitz HM, Hickey K, Garan H. Real-time stroke volume measurements for the optimization of cardiac resynchronization therapy parameters. Europace 2010; 12(9):1270-1274.

24. Scharf C, Li P, Muntwyler J, Chugh A, Oral H, Pelosi F, Morady F, Armstrong WF. Rate-dependent AV delay optimization in cardiac resynchronization therapy. Pacing Clin Electrophysiol 2005; 28(4):279-284.

25. Valzania C, Eriksson MJ, Boriani G, Gadler F. Cardiac resynchronization therapy during rest and exercise: comparison of two optimization methods. Europace 2008; 10(10):1161-1169. 
26. Valzania C, Biffi M, Martignani C, Diemberger I, Bertini M, Ziacchi M, Bacchi L, Rocchi G, Rapezzi C, Branzi A, Boriani G. Cardiac resynchronization therapy: variations in echo-guided optimized atrioventricular and interventricular delays during follow-up. Echocardiography 2007; 24(9):933939.

27. Gras D, Gupta MS, Boulogne E, Guzzo L, Abraham WT. Optimization of AV and VV delays in the real-world CRT patient population: an international survey on current clinical practice. Pacing Clin Electrophysio/ 2009; 32 Suppl 1:\$236-239.

28. Bland JM, Altman DG. Agreement between methods of measurement with multiple observations per individual. J Biopharm Stat 2007; 17(4):571-582. 
Irene E. van Geldorp 1,2

Ward Y. Vanagt ${ }^{1,2}$

Urs Bauersfeld $^{3}$

Maren Tomaske ${ }^{3}$

Frits W. Prinzen ${ }^{1}$

Tammo Delhaas ${ }^{1,2}$

${ }^{1}$ Department of Physiology, Cardiovascular Research Institute Maastricht, Maastricht University, Maastricht, The Netherlands; ${ }^{2}$ Department of Pediatrics, Division of Pediatric Cardiology, University Hospital Maastricht, Maastricht, The Netherlands; ${ }^{3}$ Department of Pediatric Cardiology, Children's University Hospital Zurich, Zurich, Switzerland 


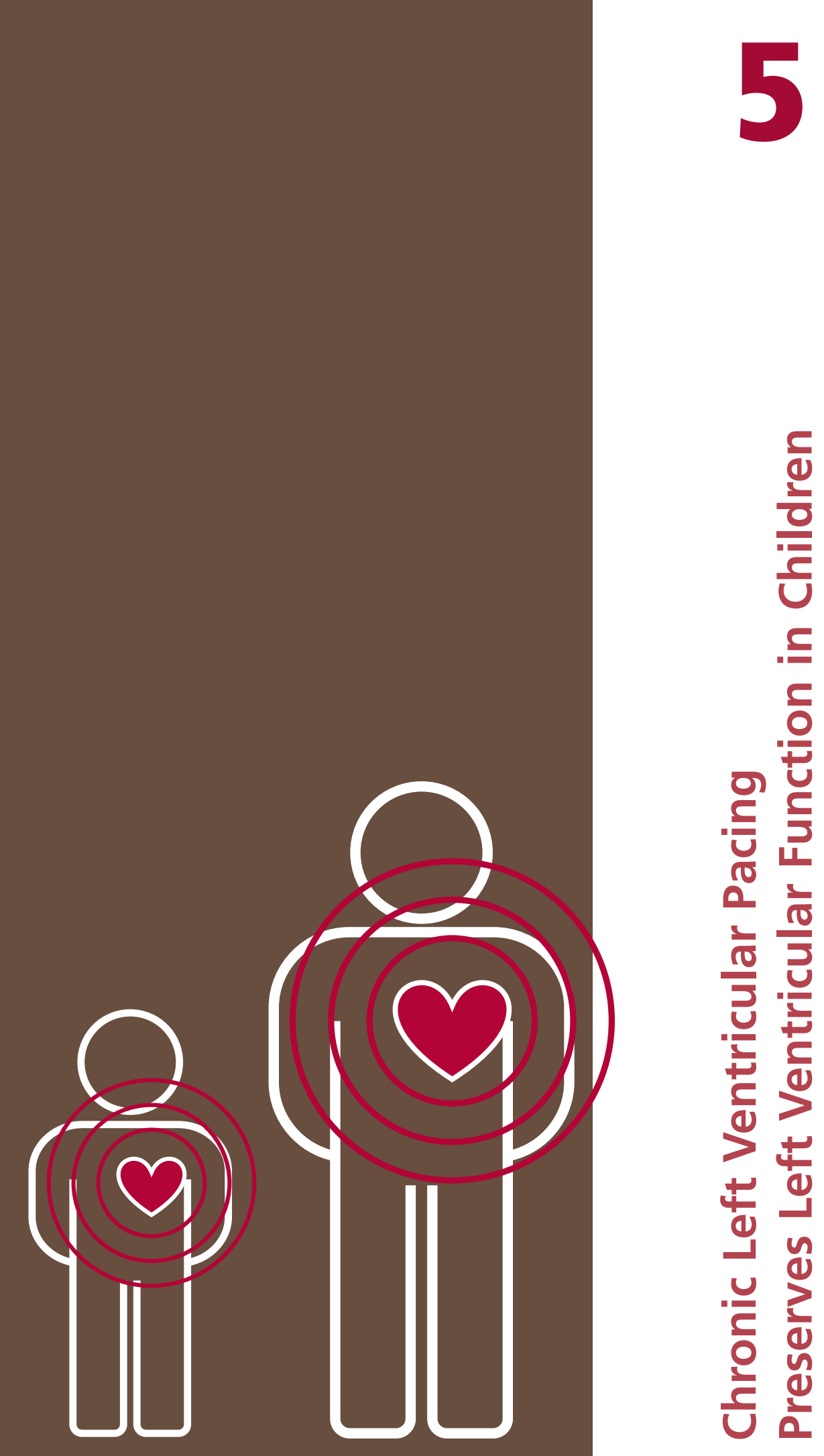




\section{Abstract}

Background: Chronic right ventricular (RV) pacing can induce structural and functional cardiac deterioration. Because animal studies showed benefit of left ventricular (LV) over RV pacing, this study compared the effects of chronic RV and LV pacing in children.

Methods: Retrospectively, echocardiographic data were evaluated from 18 healthy children (control subjects) and from children undergoing chronic epicardial RV pacing (7 RVP) or LV pacing (7 LVP). Assessment included LV end-diastolic wall thickness (LVEDWT) and end-systolic wall thickness (LVESWT) as well as LV end-diastolic diameter (LVEDD) and x-systolic diameter (LVESD). The shortening fraction (SF) and eccentricity index (=LV diameter/2 $\leftrightarrow \mathrm{LV}$ wall thickness) were calculated as measures of LV function and eccentricity, respectively. Duration of QRS and septalposterior-wall-motion-delay (SPWMD) were used as measures of electrical and mechanical dyssynchrony, respectively. A $p$ value less than 0.05 determined significance.

Results: LVEDD, LVESD, LVEDWT, and LVESWT were not significantly different between the groups. The shortening fraction was significantly lower in the RVP $(21.7 \pm 6.0 \%)$ than in the LVP $(32.2 \pm 5.2 \%)$ or control $(29.3 \pm 4.3 \%)$ children. The systolic LV eccentricity index was significantly larger in the RVP $(1.8 \pm 0.2)$ than in the LVP $(1.4 \pm 0.1)$ or control $(1.4 \pm 0.2)$ children. The SPWMD was significantly larger in the RVP $(338 \pm 20 \mathrm{~ms})$ than in the LVP $(-16 \pm 14 \mathrm{~ms})$ or control $(-5 \pm 35 \mathrm{~ms})$ group, whereas QRS-duration was similarly prolonged in the RVP $(157 \pm 10 \mathrm{~ms})$ and LVP $(158 \pm 22 \mathrm{~ms})$ groups than in the control group $(69 \pm 7 \mathrm{~ms})$.

Conclusion: Left ventricular function in children may be preserved by chronic pacing at the LV lateral wall. 


\section{INTRODUCTION}

In children and adults with congenital or acquired atrioventricular (AV) block, the ventricular pacing lead is traditionally positioned at the right ventricle (RV). ${ }^{1,}{ }^{2}$ However, RV apex pacing causes an acute decrease in left ventricular (LV) function in animals, ${ }^{3}$ adults ${ }^{4}$ and children. ${ }^{5,6}$ During chronic RV pacing in children, LV function, morphology ${ }^{7,8}$ and histology ${ }^{9}$ are at risk for deterioration over time (for review see Karpawich). ${ }^{1}$ Chronic RV pacing can eventually result in cardiac failure, which occurs in $6 \%$ to $7 \%$ of children. ${ }^{10-12}$ Also, in adults, chronic RV apex pacing has deleterious effects (for review see Manolis) ${ }^{2}$ and increases the risk of heart failure. ${ }^{13,14}$ Recognition of the possible harmful effects from RV apex pacing initiated the search for alternative ventricular pacing sites including the RV outflow tract, His bundle, LV wall, and biventricular pacing. Pacing at the His bundle is likely the superior approach, ${ }^{15}$ but appears technically difficult, especially in children. Right ventricular outflow tract pacing does not provide a consistent beneficial hemodynamic effect compared with RV apex pacing. ${ }^{4}$ During pacing at different RV septal sites, hemodynamic function varies widely, and the location of the $\mathrm{RV}$ septal site that leads to the less pronounced decrease in pump function also varies between hearts in canine experiments. ${ }^{16}$ In adults with heart failure, both LV lateral wall pacing ${ }^{17,18}$ and biventricular (RV apex + LV lateral wall) pacing ${ }^{18}$ acutely results in a better functional outcome than RV apex pacing. Also in adults, LV pacing alone seems to be as effective as biventricular resynchronization therapy, both in the acute situation ${ }^{19}$ and after 6 months of pacing. ${ }^{20}$ The current study aimed to investigate the long-term functional and structural outcome of epicardial $\mathrm{RV}$ and LV pacing in children. The study was performed retrospectively on echocardiographic and electrocardiographic (ECG) data from children undergoing chronic RV or LV pacing and from healthy children (control subjects).

\section{Methods}

\section{Study population and pacing characteristics}

All children with normal cardiac anatomy and a ventricular pacemaker in the database of the Children's University Hospital in Zurich (Switzerland) were considered for inclusion. The study included all children with congenital or acquired AV block (except for cardiomyopathy) undergoing chronic epicardial RV or LV pacing for rate control (minimum of 95\% paced beats) for whom echocardiographic and ECG data were available. Data were acquired during the most recent outpatient clinic visit of children with chronic RV pacing (RVP, $n=7$ ) or LV pacing (LVP, $n$ $=7$ ) and evaluated retrospectively. For the children who underwent a pacing lead replacement, the last echocardiography and ECG data before the replacement were evaluated to exclude effects of changes in the pacing site between the initiation of pacing and the moment the data were obtained. In addition, for a small number of patients ( 3 RVP and 4 LVP), the 
echocardiographic preimplantation data were of sufficient quality for assessment and are presented to provide an estimation of the baseline characteristics. Bipolar steroid-eluting pacing leads (Medtronic CapSure Epi 10366 or 4968; Medtronic Inc., Minneapolis, MN, USA) were implanted in all children and connected to various pulse generators. Pacemaker lead positioning was purely determined by the surgical approach preferred by surgeons. ${ }^{21}$ Through a sternotomy or using a subxyphoidal approach, RV pacing leads were implanted and positioned at the RV apex $(n=6)$ or RV free wall $(n=1)$. Left ventricular pacing leads were implanted through a left lateral thoracotomy and placed at the LV mid lateral wall. ${ }^{21}$ Table 1 depicts the characteristics of the paced children. Before pacemaker implantation and during the follow-up, none of the patients had clinical symptoms of heart failure, received any cardiovascular drugs, or had echocardiographic signs of cardiac failure. The control group consisted of 18 healthy children who visited the outpatient clinic for an innocent cardiac murmur. This retrospective study was approved by the local ethics committee and performed according to their guidelines.

Table 1: Patient and pacemaker characteristics

\begin{tabular}{|c|c|c|c|c|c|c|c|}
\hline Patient & $\begin{array}{c}\text { Sex } \\
\text { (M/F) }\end{array}$ & Pacing indication & $\begin{array}{c}\text { SPWMD (ms) } \\
\text { prepacing }\end{array}$ & $\begin{array}{c}\text { Shortening } \\
\text { Fraction (\%) } \\
\text { prepacing }\end{array}$ & $\begin{array}{l}\text { Pacing } \\
\text { mode }\end{array}$ & $\begin{array}{c}\text { Pacing } \\
\text { Duration (y) }\end{array}$ & $\begin{array}{l}\text { Age at end } \\
\text { of study (y) }\end{array}$ \\
\hline RVP-1 & $F$ & C-AVB III & & & DDD & 6.9 & 8.7 \\
\hline RVP-2 & M & C-AVB III & 70 & 28 & DDD & 2.2 & 9.4 \\
\hline RVP-3 & $\mathrm{F}$ & C-AVB III (Mat. SLE) & & & VVI & 6.3 & 6.3 \\
\hline RVP-4 & $F$ & A-AVB $\|$ & 65 & 38 & DDD & 2.5 & 4.3 \\
\hline RVP-5 & $F$ & C-AVB III & & & DDD & 2.3 & 4.4 \\
\hline RVP-6 & $F$ & C-AVB II & 10 & 40 & DDD & 2.5 & 8.4 \\
\hline RVP-7 & $\mathrm{F}$ & C-AVB III (Mat. SLE) & & & VVI & 4.9 & 4.9 \\
\hline LVP-1 & M & C-AVB III & & & VVI & 5.5 & 6.8 \\
\hline LVP-2 & $\mathrm{F}$ & A-AVB III & -5 & 40 & DDD & 1.5 & 11.1 \\
\hline LVP-3 & $F$ & C-AVB II, long QT & & & DDD & 4.8 & 4.4 \\
\hline LVP-4 & M & AVB II, SSS & 45 & 35 & DDD & 3.3 & 7.4 \\
\hline LVP-5 & M & C-AVB III & 20 & 39 & DDD & 2.5 & 3.7 \\
\hline LVP-6 & $\mathrm{F}$ & C-AVB III & 35 & 33 & DDD & 6.3 & 10.6 \\
\hline LVP-7 & M & C-AVB III (Mat. SLE) & & & VVI & 2.8 & 2.8 \\
\hline \multicolumn{8}{|c|}{ 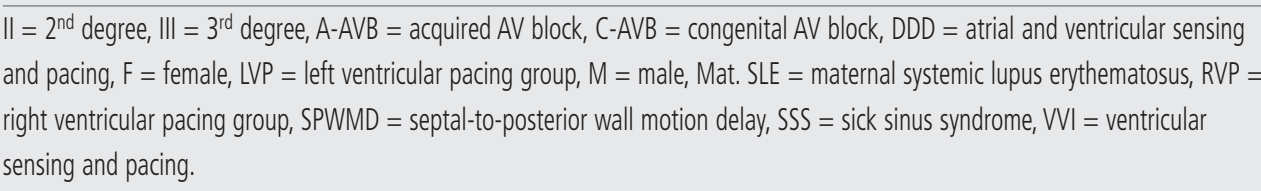 } \\
\hline vailable & & and CDWM D d ta & & & & & \\
\hline
\end{tabular}




\section{Echocardiographic evaluation}

Echocardiographic data were obtained in the standard precordial positions with appropriate transducers (7.5-, 5.0-, 3.5-, and 2.5-MHz; Vivid 7; General Electric Healthcare, UK; or Philips Sonos 5500; Philips, Best, The Netherlands). Data were stored either digitally or on VHS videotapes, then subsequently digitized offline. End-diastolic and end-systolic frames of the parasternal short-axis views were further analyzed using Matlab software (The Mathworks Inc., Natick, MA, USA). Two observers, blinded to the study group of the patient, each performed three independent measurements in random order for every patient. The average of the measurements performed by the two observers was used for further analysis. A representative example of a processed echocardiographic image is depicted in Figure 1. Body surface area was calculated using the formula of Du Bois and Du Bois ${ }^{22}$ : Body surface area $\left(\mathrm{m}^{2}\right)=0.20247 \times$ length $(\mathrm{m})^{0.725} \times$ weight $(\mathrm{kg})^{0.425}$ Left ventricular end-diastolic diameter (LVEDD) and LV end-systolic diameter (LVESD) were measured bidirectionally (Figure 1) and averaged to minimize over- or underestimation in case of an asymmetric mechanical activation pattern. To estimate the degree of LV dilatation and to compare between patients of different ages and weights, LVEDD was expressed as a z-score of normal. ${ }^{23}$ Shortening fraction (SF) as a measure of cardiac function was defined as: SF (\%) = (LVEDD - LVESD) / LVEDD x 100. Regional changes in LV wall thickness were assessed by measuring the LV wall in six consecutive regions at end-diastole and end-systole on short-axis views (Figure 1). The mean of these six regions was used as the mean LV end-diastolic wall thickness (LVEDWT) and mean LV end-systolic wall thickness (LVESWT). Again, LVEDWT was expressed as z-score of normal. The eccentricity index of the LV (as a measure of fiber stress) was calculated as follows: LV eccentricity index $=\mathrm{LV}$ diameter / ( $2 \times$ mean LV wall thickness). Septal-to-posterior wall motion delay (SPWMD), as a measure of intraventricular mechanical synchrony, was obtained from shortaxis M-mode echocardiographic images and defined as the time delay between the earliest peak of systolic inward movement of the septum and the opposite LV posterior wall. ${ }^{24-26}$

\section{Electrocardiographic evaluation}

Duration of QRS was assessed from the surface ECG during sinus rhythm (control group), RV pacing (RVP group), and LV pacing (LVP group) and used as a measure for synchrony of electrical ventricular activation.

\section{Statistical analysis}

Statistical analysis was performed using one-way analysis of variance (ANOVA) on every parameter. Only if ANOVA showed a significant difference was further analysis with Tukey comparison used to identify statistical differences between the different study groups. A p value less than 0.05 determined statistical significance. Data are presented as mean \pm standard deviation. For echocardiographic measures, the interobserver correlation ( $r$ value) was assessed using Pearson product-moment correlation. 


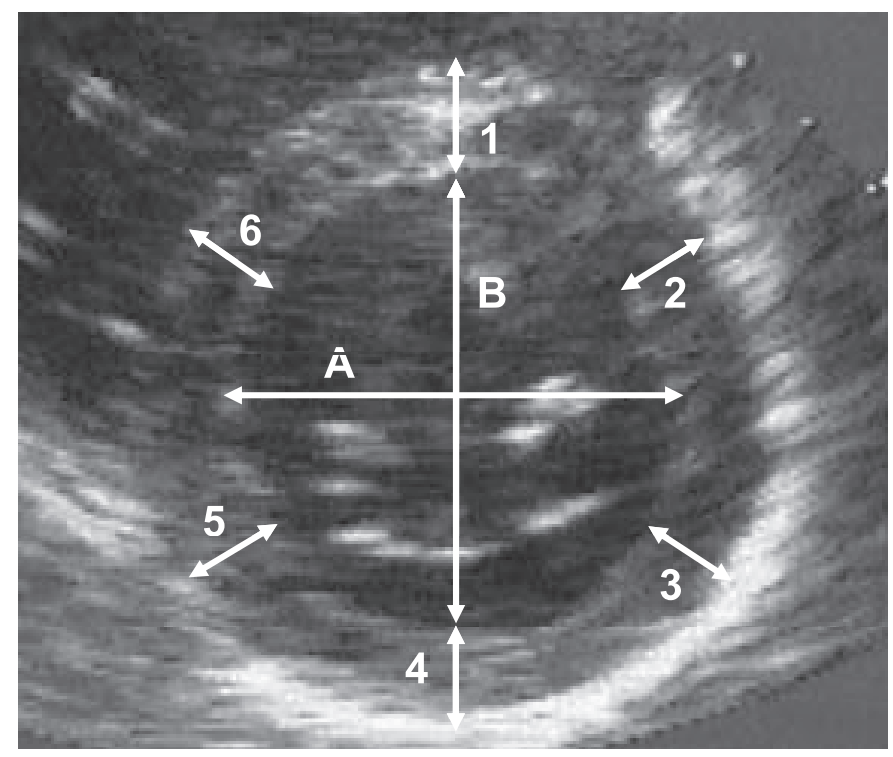

Figure 1: Representative echocardiographic short-axis view.

Six regions are indicated for wall thickness determination (arrows) and the inner diameter of the left ventricle (LV). The LV diameter was determined bidirectionally as the mean of diameters $\mathbf{A}$ and $\mathbf{B}$.

\section{RESULTS}

\section{Study population}

The characteristics of the RVP and LVP groups are presented in Table 1. Unless specified, the etiology of the AV block was not identified. Age and body surface area were not significantly different between the groups (RVP group: $6.6 \pm 2.2$ years and $0.9 \pm 0.2 \mathrm{~m}^{2}$, respectively; LVP group: $6.7 \pm 3.3$ years and $0.9 \pm 0.3 \mathrm{~m}^{2}$; control group: $4.8 \pm 3.7$ years and $0.7 \pm 0.3 \mathrm{~m}^{2}$ ). The duration of ventricular pacing was not significantly different between the two paced groups (RVP: $3.9 \pm 2.1$ years vs LVP: $3.8 \pm 1.8$ years).

Due to the paucity of preimplantation echocardiographic data, no statistical analysis was performed on these data. It seems likely from available preimplantation echocardiographies (3 RVP and 4 LVP) that mechanical asynchrony during AV nodal escape rhythm was irrelevant because SPWMD was $48 \pm 33 \mathrm{~ms}$ in the RVP group and $23 \pm 22 \mathrm{~ms}$ in the LVP group. Furthermore, available data suggest no preimplantation difference in shortening fraction between the two groups (RVP: $35.4 \% \pm 6.2 \%$ vs LVP: $36.5 \% \pm 3.3 \%$ ). 


\section{Structural echocardiographic outcome}

Interobserver reproducibility was good for LVEDD $(r=0.96)$, LVESD $(r=0.95)$, mean LVEDWT ( $r$ $=0.90)$, and mean LVESWT $(r=0.94)$. Among the three study groups, LVEDD, LVESD, mean LVEDWT, and mean LVESWT did not differ significantly (Table 2). No significant differences in LV wall thickness between different regions of the heart were observed in any of the study groups (data not shown).

Table 2: Echocardiographic structural results

\begin{tabular}{cccc} 
& RV Pacing group & LV Pacing group & Control group \\
\hline LVEDD $(\mathrm{mm})$ & $39.5 \pm 6.8$ & $39.4 \pm 6.1$ & $34.2 \pm 6.8$ \\
z-score & $0.6 \pm 0.9$ & $0.4 \pm 0.7$ & $0.0 \pm 0.9$ \\
\hline LVESD $(\mathrm{mm})$ & $31.0 \pm 6.9$ & $26.7 \pm 4.3$ & $24.2 \pm 5.2$ \\
\hline LVEDWT $(\mathrm{mm})$ & $7.4 \pm 2.1$ & $7.4 \pm 1.3$ & $6.3 \pm 1.2$ \\
z-score & $1.5 \pm 1.4$ & $1.3 \pm 0.9$ & $0.8 \pm 0.5$ \\
\hline LVESWT $(\mathrm{mm})$ & $8.7 \pm 1.9$ & $9.2 \pm 1.3$ & $8.2 \pm 1.4$ \\
\hline
\end{tabular}

$L V=$ left ventricular, LVEDD $=$ LV end-diastolic diameter, LVEDWT = mean LV end-diastolic wall thickness, LVESD = LV end-systolic diameter, LVESWT = mean LV end-systolic wall thickness, RV = right ventricular.

There are no significant differences between groups.

\section{Functional echocardiographic outcome}

After chronic epicardial pacing, the shortening fraction was significantly lower in the RVP group $(21.7 \% \pm 6.0 \%)$ than in the LVP group $(32.2 \% \pm 5.2 \%)$ or the control group $(29.3 \% \pm 4.3 \%$, nonsignificant difference between the control and LVP groups, Figure 2). The end-systolic LV eccentricity index was significantly higher in the RVP group $(1.8 \pm 0.2)$ than in the LVP $(1.4 \pm 0.1)$ and control (1.4 \pm 0.2$)$ group (nonsignificant difference between the LVP and control groups, Figure 3). At end-diastole, the LV eccentricity index was not significantly different between the study groups (RVP: $2.7 \pm 0.3$; LVP: $2.7 \pm 0.6$; control: $2.7 \pm 0.4$ ). The SPWMD was similar in the control $(-5 \pm 35 \mathrm{~ms})$ and LVP $(-16 \pm 14 \mathrm{~ms})$ groups, with good interobserver reproducibility $(r=$ 0.97). As compared with the control and LVP groups, the SPWMD in the RVP group $(338 \pm 20$ ms) was significantly different $(p<0.001)$.

\section{QRS-duration}

The duration of QRS was $69 \pm 7 \mathrm{~ms}$ in the control group, and significantly longer $(p<0.001)$ in both paced groups (RVP: $157 \pm 10$ ms vs LVP: $158 \pm 22$ ms; nonsignificant difference between RVP and LVP). 


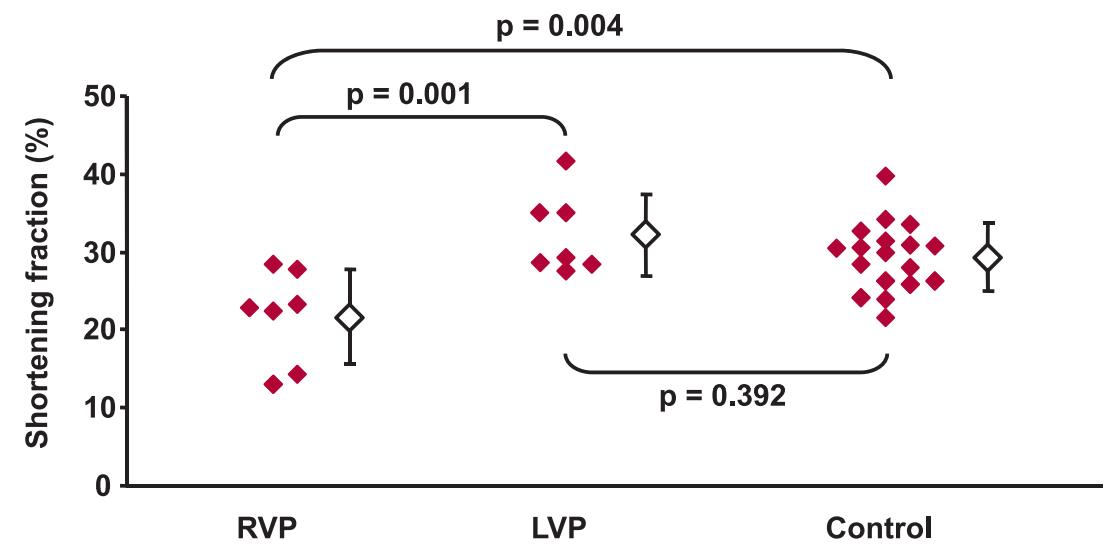

Figure 2: Shortening fraction in the study groups.

Closed symbols represent individual data. Open symbols represent group average \pm standard deviation. LVP, left ventricular pacing group; RVP, right ventricular pacing group.

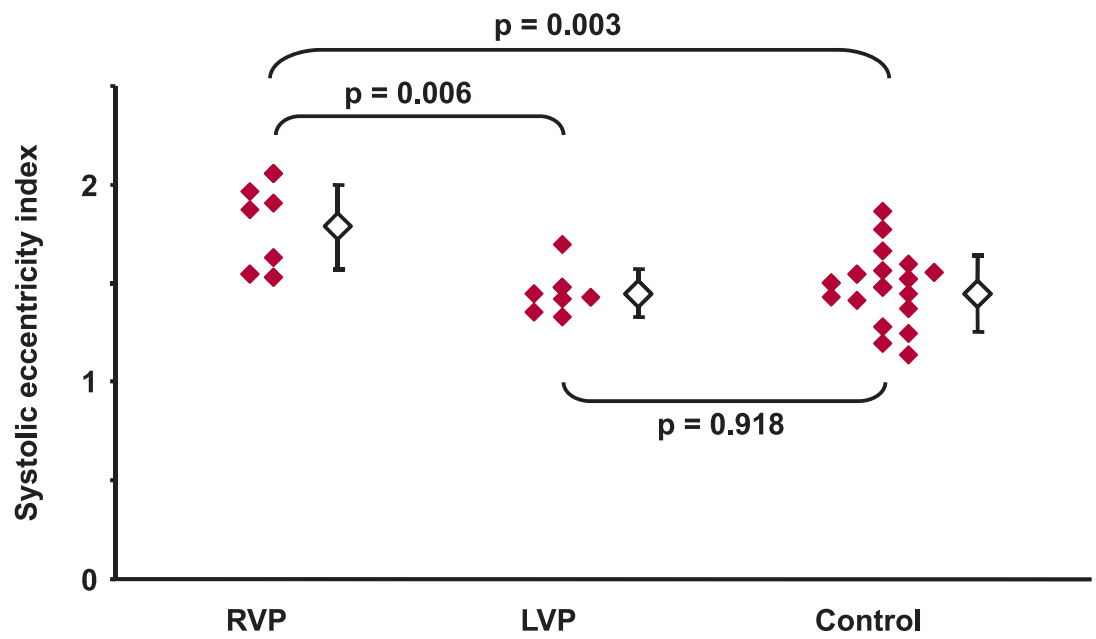

Figure 3: End-systolic left ventricular eccentricity index in the study groups.

For legends see Fig. 2.

\section{Discussion}

To our knowledge, this is the first study to compare the long-term effects of RV and LV pacing in children. The current study supports the finding of previous studies that chronic RV pacing can 
be detrimental to LV function. ${ }^{1,2,7,8}$ Chronic RV pacing is associated with deleterious LV remodeling ${ }^{8,9}$ and decreased LV function ${ }^{7}$ in the young (for a review, see Karpawich) ${ }^{1}$ and in adults (for review see Manolis). ${ }^{2}$ More importantly, the current study shows that during chronic epicardial LV pacing (mean duration $>31 \frac{1}{2}$ years), LV function and structure are maintained at the level of healthy control children. Therefore, the pacing site appears an important determinant for cardiac function and structure in children.

\section{Pacing site and LV structure}

Animal experiments showed asymmetrical hypertrophy during pacing, ${ }^{27}$ because early-activated regions of the LV had low workload due to a low pressure gradient at this early activation phase. Low workload results in hypotrophy, whereas late-activated regions hypertrophy as they are stretched before activation and thus must perform higher myocardial work (local Frank-Starling effect). Remarkably, we did not find differences in regional wall thicknesses during pacing, possibly because the quality of images was not appropriate to distinct regional changes in wall thicknesses.

\section{Relation between pacing site and LV function}

In parallel with the acute decrease in cardiac pump function during RV pacing, ${ }^{5,6}$ chronic RV pacing significantly depressed shortening fraction in the current study. Furthermore, the LV eccentricity index was significantly increased after chronic RV pacing. This eccentricity index provides an approximation of fiber stress, since fiber stress increases proportionally to the result of the following equation ${ }^{28,29}$ : LV pressure $\mathrm{x}$ (LV cavity diameter / LV wall thickness). From this equation, it can be deduced that at similar LV pressure, an increase in LV cavity diameter or a decrease in LV wall thickness will result in higher fiber stress. Although neither LV nor aortic pressures were assessed for this study, the increased eccentricity index in RV-paced children may indicate increased fiber stress. Left ventricular pacing neither increased the eccentricity index nor decreased the shortening fraction after more than 3 years of pacing. Superior hemodynamic performance during LV pacing compared with RV pacing probably is caused by a more favorable balance between interventricular synchrony, ${ }^{30}$ intraventricular synchrony, ${ }^{6}$ and the sequence of electrical ventricular activation ${ }^{3}$ during LV pacing. The idea that the sequence of activation is an important determinant of cardiac pump function is supported by other studies as well. ${ }^{3,31}$ During RV pacing, a left bundle branch block pattern of activation is created. ${ }^{1-3,6}$ In the current study, RV-paced children showed significant intraventricular mechanical dyssynchrony, with motion of the interventricular septum toward the LV posterior wall more than 300 ms before the first peak of inward LV posterior wall movement. This motion pattern of the interventricular septum is caused by the pressure developments within each ventricle. If the interventricular septum and the LV free wall are activated after the RV free wall, RV pressure increases before LV pressure is built up. This causes early systolic bulging of the interventricular septum into the LV. ${ }^{32}$ 
Cardiac resynchronization therapy with biventricular pacing increases cardiac function after surgery for congenital heart disease compared with intrinsic activation. ${ }^{30,} 33$ Experience with chronic biventricular pacing in children is sparse, but a multi-center study showed promising results with regard to cardiac function after 4 months of resynchronization therapy, ${ }^{34}$ and biventricular pacing proved effective in the treatment of six children with RV pacing-induced heart failure. ${ }^{10}$ In adults with congestive heart failure, chronic LV lateral wall pacing (single-site, short AV delay) can be as effective as biventricular pacing. ${ }^{20,35,36}$ To our knowledge, the effect of chronic biventricular pacing in children has not been compared with LV lateral wall pacing alone.

The duration of QRS was not significantly different between the two paced groups in our study despite the significantly different hemodynamic and mechanical performance. This is consistent with other studies, in which QRS duration during pacing was not related to cardiac function. ${ }^{6,31,33}$ It is important to bear in mind that QRS duration reflects total biventricular activation time, whereas intraventricular mechanical synchrony and sequence of electrical ventricular activation ${ }^{3}$ probably are more important determinants of LV function. ${ }^{2,6}$ Given the absence of a consistent correlation between cardiac function and QRS duration in acute and chronic pacing studies, we discourage the use of QRS duration as a tool for the selection of an optimal epicardial pacing site in children.

\section{Clinical application}

We apply and advocate the use of LV pacing sites when chronic epicardial pacing is indicated in children. We do so because chronic LV pacing has proved superior to RV pacing in terms of LV function and relative systolic dimensions in the current study, and because in some case reports, RV pacing-induced heart failure was successfully treated with single-site LV pacing. ${ }^{37-39}$ However, once implantation of LV epicardial leads is started, it is of major clinical importance to know which site, apex or free wall, should be preferred. In an acute-pacing study, LV apex pacing increased pump function compared with RV pacing, whereas there was no benefit of $L V$ free wall pacing. ${ }^{6}$ Nevertheless, the current study shows that LV lateral wall pacing preserves LV function. These findings could be explained by subtle differences in LV lateral wall pacing sites. In the aforementioned study, ${ }^{6}$ the pacing lead was placed at the base of the LV lateral wall, whereas in the current study, the location of the LV pacing lead was half way between the LV apex and the base of the LV lateral wall (mid lateral wall).

The hypothesis that the exact pacing site at the LV lateral wall influences the effect of LV pacing is supported by the following experiment. In an established animal left bundle branch block (LBBB) model, ${ }^{40},{ }^{41}$ we investigated the hemodynamic improvement of four epicardial LV pacing sites. In 7 dogs with experimental LBBB, pacing was applied at four LV pacing sites: apex, apical lateral wall, mid lateral wall and base of the lateral wall, respectively. At all sites, pacing was performed at the same short AV delay to avoid fusion with intrinsic activation. The averaged maximum rate of $L V$ pressure rise ( $L V d P / d t \mid m a x)$ from all beats during one ventilation cycle, 
measured with a catheter tip manometer, was used as a measure of LV function. Compared with LBBB, LVdP/dt|max was significantly increased by pacing at the LV apex $(18 \% \pm 11 \% ; p=0.005)$, LV apical lateral wall $(11 \% \pm 6 \% ; p=0.001)$ and LV mid lateral wall $(7 \% \pm 6 \% ; p=0.020)$, whereas no significant improvement in LV function occurred during pacing at the base of the LV lateral wall $(3 \% \pm 10 \% ; p=0.413)$.

Although pacing at the LV apex caused the most pronounced improvement in LV function, it is not easy to come within reach of this particular pacing site using established endovenous or minimal surgical techniques. However, pacing at epicardial LV mid lateral wall sites also resulted in improved LV function and is easily accessible through a left lateral thoracotomy. This approach is surgically reliable and provides excellent cosmetic and functional results in children. ${ }^{21}$

\section{Study limitations}

Although the number of patients studied in the current series was small, we consider the study groups to be comparable because body surface area, age, and duration of pacing were similar, and none of the patients had structural heart disease. Because echocardiographic image quality deteriorates during long-term storage, ${ }^{42}$ we were hampered in our retrospective evaluation, especially in the evaluation of preimplantation data. We therefore could not achieve a longitudinal follow-up evaluation of all the patients and could not perform statistical analysis of echocardiographic preimplantation data. Therefore, the possibility that some of the differences between chronically RV and LV paced children were preexisting cannot entirely be excluded. The main disadvantage of this study is its retrospective design. The positive effects of single-site LV pacing observed in this study, as well as the practical advantages of single-site over multisite ventricular pacing, strongly advocate further investigation on LV pacing in prospective multicenter studies.

\section{CONCLUSION}

Left ventricular function in children is preserved by chronic pacing at the LV lateral wall, whereas chronic RV pacing causes a decrease in shortening fraction and a higher systolic eccentricity index. 


\section{Acknowledgements}

The authors gratefully acknowledge Joost Lumens, MSc at Maastricht University for his contribution to the data analysis. They thank St. Jude Medical, Veenendaal, The Netherlands, for financial support of the travel expenses related to this project. Frits Prinzen is consultant to Medtronic Inc. (Minneapolis, MN, USA) and Boston Scientific Corp. (St. Paul, MN, USA). 


\section{REFERENCES}

1. Karpawich PP. Chronic right ventricular pacing and cardiac performance: the pediatric perspective. Pacing Clin Electrophysiol 2004; 27(6 Pt 2):844-849.

2. Manolis AS. The deleterious consequences of right ventricular apical pacing: time to seek alternate site pacing. Pacing Clin Electrophysiol 2006; 29(3):298-315.

3. Prinzen FW, Peschar M. Relation between the pacing induced sequence of activation and left ventricular pump function in animals. Pacing Clin Electrophysiol 2002; 25(4 Pt 1):484-498.

4. de Cock CC, Giudici MC, Twisk JW. Comparison of the haemodynamic effects of right ventricular outflow-tract pacing with right ventricular apex pacing: a quantitative review. Europace 2003; 5(3):275-278.

5. Karpawich PP, Mital S. Comparative left ventricular function following atrial, septal, and apical single chamber heart pacing in the young. Pacing Clin Electrophysiol 1997; 20(8 Pt 1):1983-1988.

6. Vanagt WY, Verbeek XA, Delhaas T, Mertens L, Daenen WJ, Prinzen FW. The Left Ventricular Apex is the Optimal Site for Pediatric Pacing. Pacing Clin Electrophysiol 2004; 27(6P2):837-843.

7. Tantengco MV, Thomas RL, Karpawich PP. Left ventricular dysfunction after long-term right ventricular apical pacing in the young. J Am Coll Cardiol 2001; 37(8):2093-2100.

8. Thambo JB, Bordachar P, Garrigue S, Lafitte S, Sanders P, Reuter S, Girardot R, Crepin D, Reant P, Roudaut R, Jais $P$, Haissaguerre M, Clementy J, Jimenez $M$. Detrimental ventricular remodeling in patients with congenital complete heart block and chronic right ventricular apical pacing. Circulation 2004; 110(25):3766-3772.

9. Karpawich PP, Rabah R, Haas JE. Altered cardiac histology following apical right ventricular pacing in patients with congenital atrioventricular block. Pacing Clin Electrophysiol 1999; 22(9):13721377.

10. Moak JP, Hasbani K, Ramwell C, Freedenberg V, Berger JT, DiRusso G, Callahan P. Dilated cardiomyopathy following right ventricular pacing for AV block in young patients: resolution after upgrading to biventricular pacing systems. J Cardiovasc Electrophysiol 2006; 17(10):1068-1071.

11. Kim JJ, Friedman RA, Eidem BW, Cannon BC, Arora G, Smith EO, Fenrich AL, Kertesz NJ. Ventricular function and long-term pacing in children with congenital complete atrioventricular block. J Cardiovasc Electrophysiol 2007; 18(4):373-377.

12. Vatasescu R, Shalganov T, Paprika D, Kornyei L, Prodan Z, Bodor G, Szatmari A, Szili-Torok T. Evolution of left ventricular function in paediatric patients with permanent right ventricular pacing for isolated congenital heart block: a medium term follow-up. Europace 2007; 9(4):228-232.

13. Sweeney MO, Hellkamp AS, Ellenbogen KA, Greenspon AJ, Freedman RA, Lee KL, Lamas GA. Adverse effect of ventricular pacing on heart failure and atrial fibrillation among patients with normal baseline QRS duration in a clinical trial of pacemaker therapy for sinus node dysfunction. Circulation 2003; 107(23):2932-2937. 
14. Andersen HR, Nielsen JC, Thomsen PE, Thuesen L, Mortensen PT, Vesterlund T, Pedersen AK. Long-term follow-up of patients from a randomised trial of atrial versus ventricular pacing for sick-sinus syndrome. Lancet 1997; 350(9086):1210-1216.

15. Deshmukh P, Casavant DA, Romanyshyn M, Anderson K. Permanent, direct His-bundle pacing: a novel approach to cardiac pacing in patients with normal His-Purkinje activation. Circulation 2000; 101(8):869-877.

16. Peschar M, de Swart H, Michels KJ, Reneman RS, Prinzen FW. Left ventricular septal and apex pacing for optimal pump function in canine hearts. J Am Coll Cardiol 2003; 41(7):1218-1226.

17. Puggioni E, Brignole M, Gammage M, Soldati E, Bongiorni MG, Simantirakis EN, Vardas P, Gadler F, Bergfeldt L, Tomasi C, Musso G, Gasparini G, Del Rosso A. Acute comparative effect of right and left ventricular pacing in patients with permanent atrial fibrillation. J Am Coll Cardiol 2004; 43(2):234-238.

18. Blanc JJ, Etienne Y, Gilard M, Mansourati J, Munier S, Boschat J, Benditt DG, Lurie KG. Evaluation of different ventricular pacing sites in patients with severe heart failure: results of an acute hemodynamic study. Circulation 1997; 96(10):3273-3277.

19. Nelson GS, Berger RD, Fetics BJ, Talbot M, Spinelli JC, Hare JM, Kass DA. Left ventricular or biventricular pacing improves cardiac function at diminished energy cost in patients with dilated cardiomyopathy and left bundle-branch block. Circulation 2000; 102(25):3053-3059.

20. Touiza A, Etienne Y, Gilard M, Fatemi M, Mansourati J, Blanc JJ. Long-term left ventricular pacing: assessment and comparison with biventricular pacing in patients with severe congestive heart failure. J Am Coll Cardiol 2001; 38(7):1966-1970.

21. Dodge-Khatami A, Kadner A, Dave H, Rahn M, Pretre R, Bauersfeld U. Left heart atrial and ventricular epicardial pacing through a left lateral thoracotomy in children: a safe approach with excellent functional and cosmetic results. Eur J Cardiothorac Surg 2005; 28(4):541-545.

22. Du Bois $D$, Du Bois EF. A formula to estimate the approximate surface area if height and weight be known. 1916. Nutrition 1989; 5(5):303-311; discussion 312-303.

23. Daubeney PE, Blackstone EH, Weintraub RG, Slavik Z, Scanlon J, Webber SA. Relationship of the dimension of cardiac structures to body size: an echocardiographic study in normal infants and children. Cardiol Young 1999; 9(4):402-410.

24. Vernooy K, Cornelussen RNM, Verbeek XAAM, Vanagt WYR, van Hunnik A, Kuiper M, Arts T, Crijns HJGM, Prinzen FW. Cardiac resynchronization therapy cures dyssynchronopathy in canine left bundle-branch block hearts. Eur Heart J 2007; 28(17):2148-2155.

25. Pitzalis MV, lacoviello M, Romito R, Guida P, De Tommasi E, Luzzi G, Anaclerio M, Forleo C, Rizzon $P$. Ventricular asynchrony predicts a better outcome in patients with chronic heart failure receiving cardiac resynchronization therapy. J Am Coll Cardiol 2005; 45(1):65-69.

26. Pitzalis MV, lacoviello M, Romito R, Massari F, Rizzon B, Luzzi G, Guida P, Andriani A, Mastropasqua F, Rizzon P. Cardiac resynchronization therapy tailored by echocardiographic evaluation of ventricular asynchrony. J Am Coll Cardiol 2002; 40(9):1615-1622. 
27. van Oosterhout MF, Prinzen FW, Arts T, Schreuder JJ, Vanagt WY, Cleutjens JP, Reneman RS. Asynchronous electrical activation induces asymmetrical hypertrophy of the left ventricular wall. Circulation 1998; 98(6):588-595.

28. Arts T, Bovendeerd PH, Prinzen FW, Reneman RS. Relation between left ventricular cavity pressure and volume and systolic fiber stress and strain in the wall. Biophys J 1991; 59(1):93-102.

29. Delhaas T, Arts T, Bovendeerd PH, Prinzen FW, Reneman RS. Subepicardial fiber strain and stress as related to left ventricular pressure and volume. Am J Physiol 1993; 264(5 Pt 2):H1548-1559.

30. Janousek J, Vojtovic P, Hucin B, Tlaskal T, Gebauer RA, Gebauer R, Matejka T, Marek J, Reich O. Resynchronization pacing is a useful adjunct to the management of acute heart failure after surgery for congenital heart defects. Am J Cardiol 2001; 88(2):145-152.

31. Prinzen FW, Van Oosterhout MF, Vanagt WY, Storm C, Reneman RS. Optimization of ventricular function by improving the activation sequence during ventricular pacing. Pacing Clin Electrophysiol 1998; 21(11 Pt 2):2256-2260.

32. Lumens J, Delhaas T, Kirn B, Arts T. Modeling ventricular interaction: a multiscale approach from sarcomere mechanics to cardiovascular system hemodynamics. Pac Symp Biocomput 2008:378389.

33. Zimmerman FJ, Starr JP, Koenig PR, Smith P, Hijazi ZM, Bacha EA. Acute hemodynamic benefit of multisite ventricular pacing after congenital heart surgery. Ann Thorac Surg 2003; 75(6):17751780.

34. Dubin AM, Janousek J, Rhee E, Strieper MJ, Cecchin F, Law IH, Shannon KM, Temple J, Rosenthal E, Zimmerman FJ, Davis A, Karpawich PP, Al Ahmad A, Vetter VL, Kertesz NJ, Shah M, Snyder C, Stephenson E, Emmel M, Sanatani S, Kanter R, Batra A, Collins KK. Resynchronization therapy in pediatric and congenital heart disease patients: an international multicenter study. J Am Coll Cardiol 2005; 46(12):2277-2283.

35. Blanc JJ, Bertault-Valls V, Fatemi M, Gilard M, Pennec PY, Etienne Y. Midterm benefits of left univentricular pacing in patients with congestive heart failure. Circulation 2004; 109(14):17411744.

36. Etienne Y, Mansourati J, Gilard M, Valls-Bertault V, Boschat J, Benditt DG, Lurie KG, Blanc JJ. Evaluation of left ventricular based pacing in patients with congestive heart failure and atrial fibrillation. Am J Cardiol 1999; 83(7):1138-1140, A1139.

37. Takasugi $\mathrm{H}$, Watanabe $\mathrm{K}$, Ono $\mathrm{Y}$, Echigo $\mathrm{S}$. Improvement of left ventricular function after changing the pacing site in a child with isolated congenital complete atrioventricular block and dilated cardiomyopathy. Pediatr Cardiol 2005; 26(1):87-89.

38. Tissot C, Aggoun Y, Rimensberger PC, Sierra J, Kalangos A, Beghetti M, da Cruz E. Left ventricular epicardial VVI pacing for a congenital complete heart block with severe myocardial dysfunction: shall epicardial pacing wires be positioned left? Int J Cardiol 2007; 116(1):e7-9.

39. Vanagt WY, Prinzen FW, Delhaas T. Reversal of pacing-induced heart failure by left ventricular apical pacing. N Engl J Med 2007; 357(25):2637-2638. 
40. Verbeek XA, Vernooy K, Peschar M, Cornelussen RN, Prinzen FW. Intra-ventricular resynchronization for optimal left ventricular function during pacing in experimental left bundle branch block. J Am Coll Cardiol 2003; 42(3):558-567.

41. Vernooy K, Verbeek XA, Peschar M, Crijns HJ, Arts T, Cornelussen RN, Prinzen FW. Left bundle branch block induces ventricular remodelling and functional septal hypoperfusion. Eur Heart $J$ 2005; 26(1):91-98.

42. Van Bogart JWC. What can go wrong with magnetic media? Publishing Research Quarterly 1996; 12(4):65-77. 
Irene E. van Geldorp 1,2

Tammo Delhaas ${ }^{2,3}$

Roman A. Gebauer ${ }^{4}$

Patrick Frias ${ }^{5}$

Maren Tomaske ${ }^{6}$

Mark K. Friedberg ${ }^{7}$

Svjetlana Tisma-Dupanovic ${ }^{8}$

Jan Elders ${ }^{9}$

Andreas Früh 10

Fulvio Gabbarini ${ }^{11}$

Petr Kubuš ${ }^{12}$

Viera Illikova ${ }^{13}$

Sabrina Tsao ${ }^{14}$

A. Christian Blank 15

Anita Hiippala 16

Thierry Sluysmans ${ }^{17}$

Peter Karpawich 18

Sally-Ann Clur 19

Xavier Ganame 20

Kathryn K. Collins ${ }^{21}$

Gisela Dann ${ }^{22}$

Jean-Benoît Thambo 23

Conceição Trigo ${ }^{24}$

Bert Nagel ${ }^{25}$

John Papagiannis 26

Annette Rackowitz ${ }^{27}$

Jan Marek ${ }^{28}$

Jan-Hendrik Nürnberg 29

Ward Y. Vanagt $2,20,30$

Frits W. Prinzen ${ }^{30}$

Jan Janoušek ${ }^{12}$

for the Working Group for Cardiac Dysrhythmias and Electrophysiology of the Association for European Pediatric Cardiology

for numbered affiliations see end of chapter 


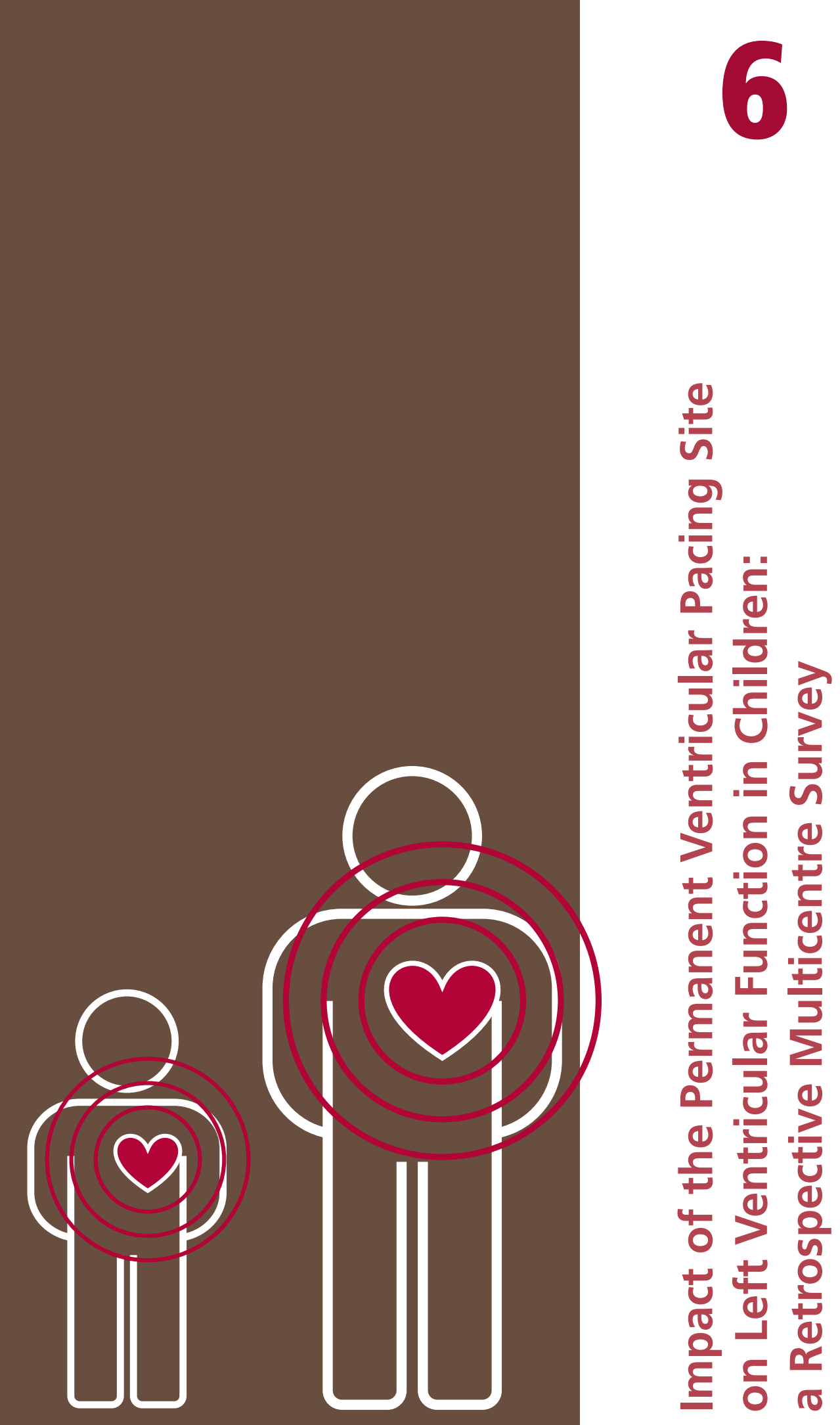




\section{Abstract}

Background: Chronic right ventricular (RV) pacing is associated with deleterious effects on cardiac function.

Objective: In an observational multicentre study in children with isolated atrioventricular (AV) block receiving chronic ventricular pacing, the importance of the ventricular pacing-site on left ventricular (LV) function was investigated.

Methods: Demographics, maternal autoantibody status and echocardiographic measurements on LV end-diastolic and end-systolic dimensions and volumes at age $<18$ years were retrospectively collected from patients undergoing chronic ventricular pacing (>1 year) for isolated AV block. LV fractional shortening (LVFS) and, if possible LV ejection fraction (LVEF) were calculated. Linear regression analyses were adjusted for patient characteristics.

Results: From 27 centres, 297 children were included in whom pacing was applied at the RV epicardium (RVepi, $n=147$ ), RV endocardium (RVendo, $n=113$ ) or LV epicardium (LVepi, $n=37$ ). LVFS was significantly affected by pacing-site $(p=0.001)$, and not by maternal autoantibody status $(p=0.266)$. LVFS in LVepi $(39 \pm 5 \%)$ was significantly higher than in RVendo $(33 \pm 7 \%$, $p<0.001)$ and RVepi $(35 \pm 8 \%, p=0.001$; no significant difference between RV-paced groups, $\mathrm{p}=0.275)$. Subnormal LVFS (LVFS $<28 \%$ ) was observed in 16/113 (14\%) RVendo-paced and 21/147 (14\%) RVepi-paced children, while LVFS was normal (LVFS $\geq 28 \%$ ) in all LVepi-paced children $(p=0.049)$. These results are supported by the findings for LVEF $(n=122)$ : LVEF was $<50 \%$ in $17 / 69$ (25\%) RVendo- and in 10/35 (29\%) RVepi-paced patients, while LVEF was $\geq 50 \%$ in $17 / 18(94 \%)$ LVepi-paced patients.

Conclusion: In children with isolated AV block, permanent ventricular pacing-site is an important determinant of LV function, with LVFS being significantly higher with LV pacing than with RV pacing. 


\section{INTRODUCTION}

In patients with bradycardia due to complete atrioventricular (AV) block, ventricular pacing is required to normalise heart rate. The pacing-induced activation pattern is characterised by a prolonged total activation duration and an abnormal sequence of activation (in both longitudinal and transverse directions). This abnormal electrical activation pattern may lead to dyssynchronous ventricular contraction, the degree of dyssynchrony varying with the site of pacing. ${ }^{1}$ Ventricular pacemaker electrodes are conventionally positioned either at the right ventricular (RV) endocardium or at the RV epicardium. However, RV pacing results in a left bundle branch block morphology and is associated with cardiac dysfunction and remodeling. ${ }^{2-7}$ The preservation of cardiac function during chronic ventricular pacing should be a high priority, especially in paediatric patients who are usually paced from an early age and may expect lifelong pacing. The main objective of this multicentre study was to investigate whether left ventricular (LV) pacing sites, in comparison with RV endocardial and RV epicardial pacing sites, have fewer adverse long-term functional and structural effects, and may prevent pacing-induced LV dysfunction in children with isolated AV block.

\section{Materials and Methods}

\section{Study population}

From the institutional databases of the participating 27 centres, patients with a structurally normal heart and isolated advanced second-degree or complete AV block with chronic and permanent ventricular pacing for rate control (minimum of 1 year follow-up, minimum of $70 \%$ ventricular paced beats) were identified. All these patients were considered for inclusion. Exclusion criteria were: postsurgical AV block, structural congenital heart disease and evident cardiomyopathy due to causes other than AV block. Study end points were reached whenever patients reached 18 years of age, underwent a change in pacing-site, received cardiac resynchronisation therapy or cardiac transplantation for heart failure, or died. For those cases, data from the last echocardiography before the event were used.

\section{Data}

Demographic data and clinical characteristics (gender, age, body surface area, aetiology of AV block, maternal autoantibody status, year of pacemaker implantation, duration of pacing) were collected. Parameters from the last echocardiography performed at a routine follow-up visit at age $<18$ years were reviewed. In all patients, LV end-diastolic diameter (LVEDD) and end-systolic diameter (LVESD) were assessed. The degree of LV dilatation was evaluated by adjusting LVEDD for body size, expressed as a z-score. ${ }^{8}$ As a measure of LV function, LV fractional shortening (LVFS) was calculated [LVFS=(LVEDD-LVESD)/LVEDD x 100\%]. According to generally accepted 
criteria, ${ }^{8,9}$ we classified LVFS as 'normal' (LVFS $\geq 28 \%$ ), 'subnormal' (LVFS $<28 \%$ ) or 'depressed' (LVFS $<25 \%$ ). For the subset of patients in whom end-diastolic and end-systolic LV volumes (LVEDV and LVESV, respectively) were assessed, LV ejection fraction (LVEF) was calculated [LVEF=(LVEDV-LVESV)/LVEDV x 100\%]. Mitral regurgitation was scored on a scale from 0 (= no regurgitation) to 4 (= severe regurgitation). Based on the location of the tip of the ventricular pacing electrode (the site of pacing), the cohort was divided into three groups: RV epicardium (RVepi), RV endocardium (RVendo), or LV epicardium (LVepi).

\section{Statistical analysis}

Comparisons between groups were performed with analysis of variance or 2 tests, as appropriate. Linear regression analyses were used to examine whether LVFS and LVEDD z-scores differed between the groups (i.e., between pacing-sites). These analyses were adjusted for the following covariates: maternal autoantibody status ('positive', 'negative', or 'unknown'), year of pacemaker implantation, age at implantation, participating centre, and duration of pacing and body surface area at echocardiographic follow-up. The influence of pacing-mode (VVI vs DDD) on LVFS was investigated only in the subset of patients for whom pacing-mode at follow-up was reported. Additionally, to carefully investigate the potential influence of maternal autoantibody status on LV function, linear regression analyses were performed on the study population grouped into 'autoantibody positive', 'autoantibody negative' and 'unknown autoantibody status'. In these analyses, 'pacing-site' was included as a covariate in addition to abovementioned characteristics. Group characteristics are expressed either as mean \pm SD, or as proportion (\%). Mean differences in LVFS and LVEDD adjusted for covariates are expressed as effect sizes (B) $95 \% \mathrm{Cls}, \mathrm{p}<0.05=$ significant.

\section{RESULTS}

\section{Study population: demographic data and clinical characteristics}

A total of 297 children, from the institutional databases of the 27 participating centres, were included in the study. Pacing-site distribution was: RVendo $(n=113)$, RVepi $(n=147)$, and LVepi $(\mathrm{n}=37)$. Maternal autoantibody status (anti-SSA (Ro), anti-SSB (La)) was reported for $201(68 \%)$ of the patients. Maternal autoantibodies were present in $88(44 \%)$ of these. Patient characteristics summarised for each pacing-site are listed in Table 1. With the above-mentioned numbers of patients in each group, it would be possible to detect differences in LVFS between the pacing-site groups with a power $>90 \%$ ( $=5 \%$ points; SD $8 \%$ points; $=0.05$; unequal groups). 
Table 1: Patient characteristics

\begin{tabular}{|c|c|c|c|c|}
\hline & $\begin{array}{l}\text { RV endo } \\
(n=113)\end{array}$ & $\begin{array}{l}\text { RV epi } \\
(n=147)\end{array}$ & $\begin{array}{l}\text { LV epi } \\
(n=37)\end{array}$ & $p$-value \\
\hline Gender (M/F; \%) & $50 / 50$ & $44 / 56$ & $46 / 54$ & 0.692 \\
\hline \multicolumn{5}{|l|}{ Aetiology of AV block } \\
\hline - Congenital / infectious / unknown (\%) & $69 / 3 / 28$ & $85 / 1 / 14$ & $92 / 0 / 8$ & 0.126 \\
\hline - Anti-Ro/La antibodies: + I - / unknown (\%) & $17 / 38 / 45$ & $40 / 43 / 17$ & $27 / 19 / 54$ & $<0.001$ \\
\hline Age at implantation (years) & $7.9 \pm 4.1$ & $2.0 \pm 2.4$ & $2.7 \pm 3.5$ & $<0.001^{*}$ \\
\hline Age at follow-up (years) & $13.8 \pm 3.4$ & $7.4 \pm 4.2$ & $7.3 \pm 4.5$ & $<0.001^{*}$ \\
\hline BSA at follow-up (m2) & $1.5 \pm 0.3$ & $0.9 \pm 0.3$ & $0.9 \pm 0.4$ & $<0.001^{*}$ \\
\hline Duration of pacing at follow-up (years) & $5.9 \pm 3.4$ & $5.4 \pm 3.6$ & $4.6 \pm 3.1$ & 0.108 \\
\hline Years since implantation until July 2010 & $8.9 \pm 4.0$ & $8.2 \pm 4.5$ & $8.1 \pm 6.1$ & 0.468 \\
\hline QRS duration (paced; ms) & $146 \pm 19$ & $139 \pm 20$ & $148 \pm 24$ & $0.004 t$ \\
\hline \multicolumn{5}{|l|}{ Pacing mode at follow-up } \\
\hline - VVI / DDD / not reported (\%) & $32 / 50 / 18$ & $46 / 40 / 14$ & $22 / 38 / 40$ & 0.059 \\
\hline
\end{tabular}

AV block = atrioventricular block; BSA = body surface area; LVepi = left ventricular epicardial pacing; RV endo = right ventricular endocardial pacing; RVepi = right ventricular epicardial pacing.

* No significant differences between RV epi and LV epi ( $p=0.229, p=0.809$ and $p=0.837$ for age at implantation, age at followup and BSA at follow-up, respectively)

$\dagger R V$ epi versus $R V$ endo, $p=0.005$; RV epi vs $L V$ epi, $p=0.012 ; R V$ endo versus $L V$ epi, $p=0.545$.

\section{Effect of pacing-site on left ventricular fractional shortening and dilatation score}

At routine follow-up, LVFS was significantly higher in children with LVepi pacing (39 $\pm 5 \%)$ than in

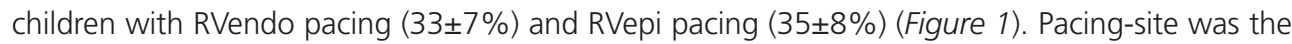
solitary significant determinant $(p=0.001)$ of LVFS (maternal autoantibody status, $p=0.266$; duration of pacing, $p=0.833$; body surface area at follow-up, $p=0.882$; centre, $p=0.560$; year of implantation, $p=0.182$; and age at implantation, $p=0.809$ ). The mean LVFS differences adjusted for covariates (i.e., 'effect size $B^{\prime}$ ) are reported in Table 2. In the subgroup of patients for whom the pacing-mode (either VVI or DDD) at follow-up was reported $(n=242)$, pacing-mode was not a significant determinant $(p=0.209)$ while pacing-site remained a significant determinant of LV function ( $p=0.002$ ). Differences between pacing-sites, adjusted for pacing-mode in addition to the other covariates, were similar to the effect sizes reported in Table 2. LVEDD z-score was normal in all groups (RVendo 0.0 \pm 1.3 ; RVepi $0.4 \pm 1.1$; LVepi $0.3 \pm 0.9$, Figure 2), and was not significantly influenced by pacing-site $(p=0.640)$, or by maternal autoantibody status $(p=0.724)$ or any of the other covariates. The adjusted means were not significantly different as presented in Table 2. 


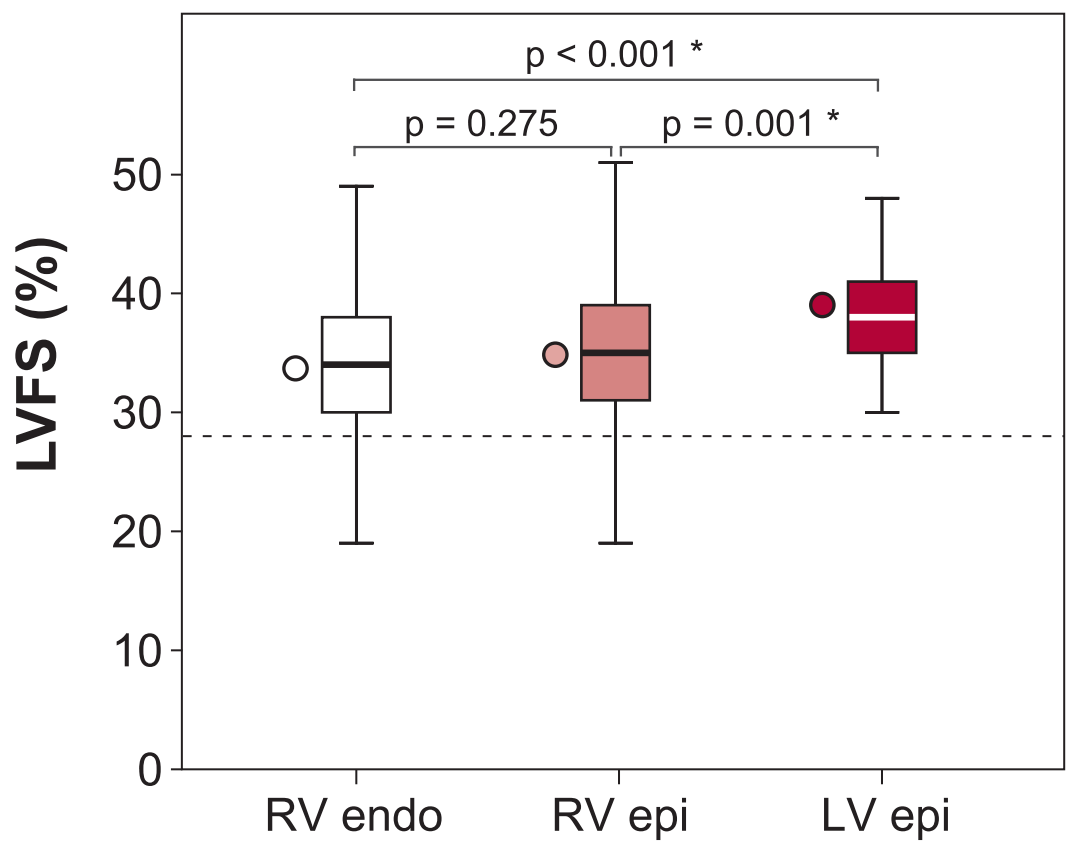

\section{pacing site}

Figure 1. Left ventricular fractional shortening after chronic ventricular pacing.

Left ventricular fractional shortening (LVFS), a measure of LV function, was significantly (*) higher in children being paced at the LV epicardium (LVepi) than in children being paced at the RV epicardium (RVepi) or RV endocardium (RVendo). Box plots represent $25^{\text {th }}, 50^{\text {th }}$, and $75^{\text {th }}$ percentiles. Whiskers represent the minimal and maximal values within the range of $\left[25^{\text {th }}\right.$ percentile $\left.-1.5^{*} \mathrm{IQR}\right]$ and $\left[75^{\text {th }}\right.$ percentile $\left.+1.5^{*} \mathrm{IQR}\right]$ respectively. Dots display mean values.

\section{Depressed LV function in $\sim 10 \%$ of the chronically RV-paced patients}

LVFS was subnormal (LVFS <28\%) in 16 RVendo- (14\%) and 21 RVepi-paced patients (14\%). In more detail, LVFS was depressed (LVFS <25\%) in 10 (9\%) of the RVendo-paced and in $17(12 \%)$ of the RVepi-paced patients. In contrast, LVFS was normal (LVFS $\geq 28 \%$ ) in all patients in whom LV pacing was applied ( $p=0.049, X^{2}$ test). In the subset of patients for whom we could calculate LVEF ( $n=122)$, LVEF was <50\% in 17/69 (25\%) RVendo-paced and in 10/35 (29\%) RVepi-paced patients, whereas this was only the case in one of the 18 (6\%) LVepi-paced patients. 
Table 2: Mean differences in left ventricular fractional shortening and dilatation score

\begin{tabular}{lccc} 
& Effect size B & $95 \%$ Cl & p-value \\
\hline LVFS & & & \\
LVepi vs RVepi & $+4.7 \%$-points & $1.9 \%$ to $7.5 \%$-points & $0.001^{*}$ \\
LVepi vs RVendo & $+6.1 \%$-points & $2.9 \%$ to $9.3 \%$-points & $<0.001^{*}$ \\
RVepi vs RVendo & $+1.4 \%$-points & $-1.1 \%$ to $3.9 \%$-points & 0.275 \\
\hline LVEDD z-score & & & \\
LVepi vs RVepi & -0.1 & -0.5 to 0.3 & 0.579 \\
LVepi vs RVendo & -0.3 & -0.8 to 0.2 & 0.268 \\
RVepi vs RVendo & -0.2 & -0.5 to 0.2 & 0.428 \\
\hline
\end{tabular}

Effect size $B=$ mean difference between groups, adjusted for covariates.

* Significant mean difference between the groups.

LVEDD = left ventricular end-diastolic diameter; LVepi = left ventricular epicardial pacing; LVFS = left ventricular fractional shortening; RVendo = right ventricular endocardial pacing; RVepi = right ventricular epicardial pacing.

\section{Mitral regurgitation}

Distribution of mitral regurgitation scores was significantly different between the pacing-site groups ( $p=0.032, X^{2}$ test). Mitral regurgitation was mild (score 1$)$ in $42(29 \%)$ RVepi-paced and in $19(17 \%)$ RVendo-paced patients, but only in three (8\%) LVepi-paced patients. Score ' 2 ' was reported for four patients (RVepi $n=1$; RVendo $n=2$, LVepi $n=1$ ), while none of the patients had moderate to severe mitral regurgitation (scores 3 and 4 ).

\section{Influence of maternal autoantibody status}

As shown by the analyses grouped by pacing-site, LVFS was significantly affected by pacing-site ( $p=0.001$ ), while maternal autoantibody status did not affect LVFS, or LVEDD z-score $(p=0.266$ and $p=0.724$, respectively). These results were confirmed when analyses were performed on the same population grouped by maternal autoantibody status. For the three groups, mean LVFS and LVEDD z-scores were comparable: $34 \pm 8 \%$ and $0.4 \pm 1.3$ for 'positive', $35 \pm 7 \%$ and $0.1 \pm 0.9$ for 'negative', and $34 \pm 8 \%$ and $0.1 \pm 1.3$ for 'unknown' maternal autoantibody status (LVFS and LVEDD z-score, respectively). Maternal autoantibody status was not a significant determinant of either LVFS $(p=0.386)$ or LVEDD $z$-score $(p=0.901)$, while pacing-site remained a significant determinant of LVFS $(p=0.013)$. 


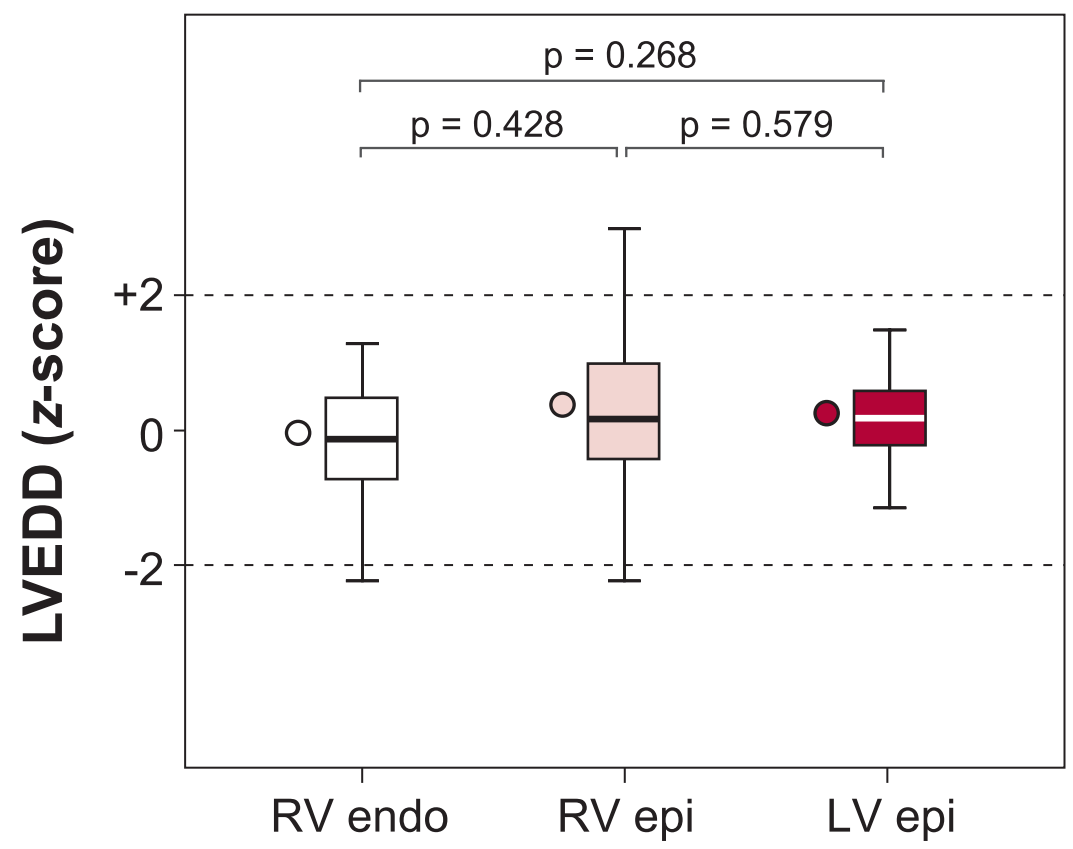

\section{pacing site}

Figure 2. Left ventricular dilatation-score after chronic ventricular pacing

The z-score for left ventricular end-diastolic diameter (LVEDD z-score), a measure of left ventricular dilatation, was not significantly influenced by the site of pacing. LVEDD z-score was normal for all groups and differences between the pacing groups were not significant. Box plots represent $25^{\text {th }}, 50^{\text {th }}$, and $75^{\text {th }}$ percentiles. Whiskers represent the minimal and maximal values within the range of $\left[25^{\text {th }}\right.$ percentile $\left.1.5^{*} \mathrm{IQR}\right]$ and $\left[75^{\text {th }}\right.$ percentile $\left.+1.5^{*} \mathrm{IQR}\right]$, respectively. Dots display mean values.

\section{Discussion}

This multicentre study retrospectively surveys LV function and dimensions in 297 children with structurally normal hearts and chronic ventricular pacing for isolated AV block. In these patients, requiring lifelong chronic ventricular pacing, preservation of cardiac functional and structural integrity is a major challenge. This study indicates that pacing-site significantly influences LVFS, with better LVFS in LVepi-paced patients than in RVepi-paced or RVendo-paced patients. 


\section{Pacing-site influences left ventricular function}

In this survey, LV function was found to be subnormal (LVFS $<28 \%$ ) in 14\%, and depressed (LVFS $<25 \%$ ) in $\sim 10 \%$ of the RVepi- and endo-paced patients. Our findings on LVFS are supported by those in the subset of patients for whom we were able to calculate LVEF: the proportion of patients with subnormal LVEF (LVEF <50\%) was for each pacing-group comparable to the proportion of patients with subnormal LVFS. Several other studies report that, within less than a decade of pacing, 7-10\% of chronically RV-paced patients develop heart failure and that up to $13 \%$ have depressed LV function combined with LV dilatation. ${ }^{2}$ 10, ${ }^{11}$ Chronic RV pacing, rather than the aetiology of $\mathrm{AV}$ block, has been identified as an independent risk factor for development of LV dilatation and dysfunction. ${ }^{11,12}$ This study indicates that it is predominantly the pacing-site that affects LV function, as reflected by (1) a higher LVFS in the LV-paced group than in the RV-paced groups; (2) pacing site being the only significant factor influencing LVFS; and (3) dissimilar incidence of patients with LVFS $<28 \%$ between the pacing-site groups. Moreover, maternal autoantibody status did not significantly influence LV function or LV dilatation score. Though it appears that there are relevant proportions of patients who do not tolerate RV pacing, the reasons are not elucidated in this retrospective study. Chronic LV pacing, however, seems to be well tolerated by all patients, as suggested by absence of LV dysfunction in the LV-paced group. From the latter finding we hypothesise that pacing-induced LV failure in children with structurally normal hearts might be prevented by LV pacing.

\section{Potential reasons for preservation of LV function by LV pacing}

We postulate that, above and beyond synchrony (reflected by the total duration of activation), the sequence of activation is a major determinant of cardiac pump function. ${ }^{1,13}$ In LV pacing the pattern of activation and mechanical contraction pattern may be more favourable than patterns induced by RV pacing. During LV (free wall) pacing, the total duration of activation is prolonged similarly to that during RV free wall pacing, reflected by a similar QRS duration. ${ }^{14}$ However, in contrast to RV pacing, LV pacing leads to activation of the LV lateral wall before the septum and RV lateral wall, preventing paradoxical septal movement and resulting in better haemodynamic performance than with RV pacing. ${ }^{1}{ }^{15}$ Furthermore, LV apical pacing induces physiological apex-to-base activation, which results in synchronous electrical activation and contraction around the circumference of the LV. ${ }^{16,17}$ These remarks are supported by the observation of Gebauer et al., that LV apical pacing preserves septal-tolateral LV mechanical synchrony as well as systolic function. ${ }^{18}$ Also, experimental studies have shown that LV apical pacing is better than pacing at other sites and that it maintains cardiac function at a normal level. ${ }^{19,20}$

\section{Single-site left ventricular pacing versus biventricular pacing}

Biventricular pacing is often used to resynchronise electrical activation in patients with either intrinsic or pacing-induced dyssynchrony and LV dysfunction. Biventricular pacing (following 
chronic RV pacing) improves pump function and reverses ventricular remodelling in children at least as effectively as in adults with heart failure. ${ }^{21-23}$ Although single-site LV pacing and biventricular pacing have not yet been compared in children, single-site LV pacing in adult patients with heart failure results in the same improvement in LV function as acute or chronic biventricular pacing. ${ }^{24-26}$ Also, animal experiments have indicated that single-site LV apical and LV septal pacing maintain cardiac function and efficiency at least as well as biventricular pacing. ${ }^{19}$ The use of a single optimal ventricular pacing-site provides advantages over biventricular pacing, such as lower pacemaker battery usage and a reduction in the number of surgical access routes required and consequent scar tissue formation.

\section{Clinical implications of the results from the current study}

Since paediatric patients with AV block are usually paced from an early age and require lifelong pacing, preservation of cardiac function during chronic ventricular pacing is important. This study indicates that LV pacing may be better than RV pacing if LV function is concerned. In the individual patient, depressed LV function (as seen in $\sim 10 \%$ of the RV-paced patients) may indicate that chronic pacing is not well tolerated and that there may be a higher risk for pacinginduced heart failure. The median follow-up of pacing in this study, as in other studies, was less than a decade, which is a mere fraction of the lifelong follow-up expectancy of a child receiving ventricular pacing for complete AV block. The (very) long-term outcome of either RV or LV pacing beginning in childhood is still unknown. Considering the findings of this and other studies, ${ }^{14,27}$, ${ }^{28}$ we suggest the use of a single LV apex or LV free wall site for chronic ventricular pacing in children with AV block and structurally normal hearts. Unfortunately, as each ventricle brings a continuum of possible pacing-sites, and accuracy of (retrospective) determination of the precise site is limited, this survey has not provided exact data to reliably test which spot on the left ventricle would be the best, or whether certain sites within the right ventricle may be better than others. ${ }^{7}$ It is important not to simply extrapolate the results of this study to children with structural congenital heart disease. Nevertheless, the results are likely to be applicable to patients with a systemic left ventricle, in absence of intrinsic RV activation delay.

\section{Practical considerations}

Surgical access for LV pacing via a left lateral thoracotomy is minimal, easy and safe, though invasive. ${ }^{29}$ In small children, the LV apex can easily be reached by a sub-xiphoidal approach, thereby avoiding a lateral thoracotomy, and with reasonable cosmetic results. Acknowledging the potential surgical complications, we particularly suggest implantation of electrodes at the LV epicardium if there are also other indications for a surgical approach. ${ }^{30}$ In larger children, singlesite epicardial LV pacing may also be achieved by a transvenous approach via the coronary sinus. In the near future, endocardial pacing in the systemic ventricle may become feasible through the application of 'wireless pacing'. However, in practice, the routine transvenous approach seems 
justifiable in young adults, as RV apical pacing is well tolerated by most patients. ${ }^{10,} 31$ Regular follow-up with echocardiography to detect LV deterioration at an early stage is warranted in all paced patients receiving chronic ventricular pacing. Changing to either biventricular or single-site pacing at the systemic ventricle should be considered when echocardiography discloses signs of progressive ventricular dysfunction. ${ }^{21,22,32-34}$

\section{Study limitations}

The retrospective design of the study is a disadvantage, mainly because it hampers the use of sophisticated echocardiographic parameters. The shortening fraction is a limited marker for systolic function, and it may be affected by intraventricular asynchrony. Nevertheless, it was chosen as a main outcome parameter, as it is the most consistently measured variable in this population. Data on LVFS in the entire study population were supported by data on LVEF in those patients for whom LV volumes were available. The possibility of unintended bias for the effect of pacing from the various sites cannot totally be excluded since measurements were not performed blinded to the pacing-site. However, at the time of the echocardiographic examination, there was no intention to compare the effects of different pacing-sites. Each centre identified patients eligible for the study by systematically reviewing the institutional database. The structure of the database affected the time span of inclusion, and therefore also the number of patients from each centre. The number of LV-paced patients included in this study is relatively small in respect to the number of patients in the RV-paced groups, since LV pacing (for first implantation) is only used at a minority of centres. However, patients from several different centres are included for each pacing group (nine centres for the LV-paced group). Besides, 'participating centre' was entered as a covariate in the analysis to correct for potential bias arising from centre-based differences.

Future studies with larger numbers of LV-paced patients, more sophisticated echocardiographic indices and longitudinal follow-up are needed to confirm the conclusions of this survey, and to explore the effect of pacing-site on parameters other than LV function.

\section{Conclusion}

In children with normal cardiac anatomy and AV block, the site of pacing is an important determinant of LV function, with LVFS being significantly higher in children with chronic LV pacing than in children with chronic RV pacing. LVFS was subnormal (LVFS $<28 \%$ ) in $14 \%$ of the RV-paced children, whereas LVFS was normal in all LV-paced children. 


\section{Funding}

JJ was supported by the research project of Universtiy Hospital Motol MZOFNM2005.

\section{Competing interests}

None

\section{Ethics approval}

Maastricht University Medical Center 


\section{Author affiliations}

${ }^{1}$ Department of Cardiology, Cardiovascular Research Institute Maastricht, Maastricht University Medical Center, Maastricht, The Netherlands; ${ }^{2}$ Department of Pediatrics, Maastricht University Medical Center, Maastricht, The Netherlands; ${ }^{3}$ Department of Biomedical Engineering, Cardiovascular Research Institute Maastricht, Maastricht University, Maastricht, The Netherlands; ${ }^{4}$ Department of Pediatric Cardiology, Heart Center, University of Leipzig, Leipzig, Germany; ${ }^{5}$ Sibley Heart Center, Children's Healthcare of Atlanta, Emory University School of Medicine, Atlanta, USA; ${ }^{6}$ Paediatric Cardiology, University Children's Hospital, Zürich, Switzerland; ${ }^{7}$ Devision of Cardiology, Hospital for Sick Children, Toronto, Canada; ${ }^{8}$ Department of Cardiology, Children's Mercy Hospital, Kansas City, USA; ${ }^{9}$ Department of Pediatric Cardiology, Universitair Medisch Centrum St. Radboud, Nijmegen, The Netherlands; ${ }^{10}$ Department of Pediatric Cardiology, Oslo University Hospital, Oslo, Norway; ${ }^{11}$ Department of Pediatric Cardiology, Ospedale Infantile Regina Margherita, Turin, Italy; ${ }^{12}$ Cardiocentrum and Cardiovascular Research Center, University Hospital Motol, Prague, Czech Republic; ${ }^{13}$ Department of Pediatric Cardiology, Children's Cardiac Center, Bratislava, Slovakia; ${ }^{14}$ Department of Electrophysiology, Children's Memorial Hospital, Chicago, USA; ${ }^{15}$ Department of Pediatric Cardiology, Wilhelmina Kinderziekenhuis, University Medical Center, Utrecht, The Netherlands; ${ }^{16}$ Department of Pediatric Cardiology, Hospital for Children and Adolescents, Helsinki University Hospital, Helsinki, Finland; ${ }^{17}$ Department of Pediatric and Congenital Cardiology, Clinique Universitaire Saint-Luc, Brussels, Belgium; ${ }^{18}$ Department of Cardiac Electrophysiology, Children's Hospital of Michigan, Wayne State University School of Medicine, Detroit, USA; ${ }^{19}$ Department of Pediatric Cardiology, Emma Kinderziekenhuis, Academisch Medisch Centrum, Amsterdam, The Netherlands; ${ }^{20}$ Department of Pediatric Cardiology, University Hospital Gasthuisberg, Leuven, Belgium; ${ }^{21}$ Department of Pediatric Cardiology, Children's Hospital University of Colorado, Denver, USA; ${ }^{22}$ Department of Pediatric Cardiology, University Hospital Göttingen, Göttingen, Germany; ${ }^{23}$ Department of Congenital Heart Disease, Hospital Haut-Lévêque, Bordeaux University, PessacBordeaux, France; ${ }^{24}$ Department of Pediatric Cardiology, Santa Marta Hospital, Lisbon, Portugal; ${ }^{25}$ Department of Pediatric Cardiology, University Children's Hospital, Graz, Austria; ${ }^{26}$ Department of Pediatric Cardiology, Mitera Children's Hospital, Maroussi, Greece; ${ }^{27}$ Department of Pediatric Cardiology, Sophia Kinderziekenhuis, Erasmus University Medical Center, Rotterdam, The Netherlands; ${ }^{28}$ Department of Cardiothoracics, Great Ormond Street Hospital, London, United Kingdom; ${ }^{29}$ Department of Congenital Heart Disease and Pediatric Cardiology, Klinikum Links der Weser, Bremen, Germany; ${ }^{30}$ Department of Physiology, Cardiovascular Research Institute Maastricht, Maastricht University, Maastricht, The Netherlands 
Chapter 6 


\section{REFERENCES}

1. Prinzen FW, Peschar M. Relation between the pacing induced sequence of activation and left ventricular pump function in animals. Pacing Clin Electrophysiol 2002; 25(4 Pt 1):484-498.

2. Moak JP, Barron KS, Hougen TJ, Wiles HB, Balaji S, Sreeram N, Cohen MH, Nordenberg A, Van Hare GF, Friedman RA, Perez M, Cecchin F, Schneider DS, Nehgme RA, Buyon JP. Congenital heart block: development of late-onset cardiomyopathy, a previously underappreciated sequela. J Am Coll Cardiol 2001; 37(1):238-242.

3. Tantengco MV, Thomas RL, Karpawich PP. Left ventricular dysfunction after long-term right ventricular apical pacing in the young. J Am Coll Cardiol 2001; 37(8):2093-2100.

4. Karpawich PP. Chronic right ventricular pacing and cardiac performance: the pediatric perspective. Pacing Clin Electrophysiol 2004; 27(6 Pt 2):844-849.

5. Thambo JB, Bordachar P, Garrigue S, Lafitte S, Sanders P, Reuter S, Girardot R, Crepin D, Reant P, Roudaut R, Jais $P$, Haissaguerre M, Clementy J, Jimenez $M$. Detrimental ventricular remodeling in patients with congenital complete heart block and chronic right ventricular apical pacing. Circulation 2004; 110(25):3766-3772.

6. Janousek J, Tomek V, Chaloupecky V, Gebauer RA. Dilated cardiomyopathy associated with dualchamber pacing in infants: improvement through either left ventricular cardiac resynchronization or programming the pacemaker off allowing intrinsic normal conduction. I Cardiovasc Electrophysiol 2004; 15(4):470-474.

7. Albouaini K, Alkarmi A, Mudawi T, Gammage MD, Wright DJ. Selective site right ventricular pacing. Heart 2009; 95(24):2030-2039.

8. Overbeek LI, Kapusta L, Peer PG, de Korte CL, Thijssen JM, Daniels O. New reference values for echocardiographic dimensions of healthy Dutch children. Eur J Echocardiogr 2006; 7(2):113-121.

9. Henry WL, Gardin JM, Ware JH. Echocardiographic measurements in normal subjects from infancy to old age. Circulation 1980; 62(5):1054-1061.

10. Vatasescu R, Shalganov T, Paprika D, Kornyei L, Prodan Z, Bodor G, Szatmari A, Szili-Torok T. Evolution of left ventricular function in paediatric patients with permanent right ventricular pacing for isolated congenital heart block: a medium term follow-up. Europace 2007; 9(4):228-232.

11. Gebauer RA, Tomek V, Salameh A, Marek J, Chaloupecky V, Gebauer R, Matejka T, Vojtovic P, Janousek J. Predictors of left ventricular remodelling and failure in right ventricular pacing in the young. Eur Heart J 2009; 30(9):1097-1104.

12. Karpawich PP, Rabah R, Haas JE. Altered cardiac histology following apical right ventricular pacing in patients with congenital atrioventricular block. Pacing Clin Electrophysio/ 1999; 22(9):1372-1377.

13. Puggioni E, Brignole M, Gammage M, Soldati E, Bongiorni MG, Simantirakis EN, Vardas $P$, Gadler F, Bergfeldt L, Tomasi C, Musso G, Gasparini G, Del Rosso A. Acute comparative effect of right and left ventricular pacing in patients with permanent atrial fibrillation. J Am Coll Cardio/ 2004; 43(2):234-238. 
14. van Geldorp IE, Vanagt WY, Bauersfeld U, Tomaske M, Prinzen FW, Delhaas T. Chronic left ventricular pacing preserves left ventricular function in children. Pediatr Cardio/ 2009; 30(2):125-132.

15. Little WC, Reeves RC, Arciniegas J, Katholi RE, Rogers EW. Mechanism of abnormal interventricular septal motion during delayed left ventricular activation. Circulation 1982; 65(7):1486-1491.

16. Wyman BT, Hunter WC, Prinzen FW, Faris OP, McVeigh ER. Effects of single- and biventricular pacing on temporal and spatial dynamics of ventricular contraction. Am J Physiol Heart Circ Physiol 2002; 282(1):H372-379.

17. Peschar M, de Swart H, Michels KJ, Reneman RS, Prinzen FW. Left ventricular septal and apex pacing for optimal pump function in canine hearts. J Am Coll Cardiol 2003; 41(7):1218-1226.

18. Gebauer RA, Tomek V, Kubus P, Razek V, Matejka T, Salameh A, Kostelka M, Janousek J. Differential effects of the site of permanent epicardial pacing on left ventricular synchrony and function in the young: implications for lead placement. Europace 2009; 11(12):1654-1659.

19. Mills RW, Cornelussen RN, Mulligan LJ, Strik M, Rademakers LM, Skadsberg ND, van Hunnik A, Kuiper M, Lampert A, Delhaas T, Prinzen FW. Left ventricular septal and left ventricular apical pacing chronically maintain cardiac contractile coordination, pump function and efficiency. Circ Arrhythm Electrophysiol 2009; 2(5):571-579.

20. Vanagt WY, Verbeek XA, Delhaas T, Mertens L, Daenen WJ, Prinzen FW. The left ventricular apex is the optimal site for pediatric pacing: correlation with animal experience. Pacing Clin Electrophysio/ 2004; 27(6 Pt 2):837-843.

21. Dubin AM, Janousek J, Rhee E, Strieper MJ, Cecchin F, Law IH, Shannon KM, Temple J, Rosenthal E, Zimmerman FJ, Davis A, Karpawich PP, Al Ahmad A, Vetter VL, Kertesz NJ, Shah M, Snyder C, Stephenson E, Emmel M, Sanatani S, Kanter R, Batra A, Collins KK. Resynchronization therapy in pediatric and congenital heart disease patients: an international multicenter study. J Am Coll Cardiol 2005; 46(12):2277-2283.

22. Janousek J, Gebauer RA, Abdul-Khaliq H, Turner M, Kornyei L, Grollmuss O, Rosenthal E, Villain E, Fruh A, Paul T, Blom NA, Happonen JM, Bauersfeld U, Jacobsen JR, van den Heuvel F, Delhaas T, Papagiannis J, Trigo C. Cardiac resynchronisation therapy in paediatric and congenital heart disease: differential effects in various anatomical and functional substrates. Heart 2009; 95(14):1165-1171.

23. Cecchin F, Frangini PA, Brown DW, Fynn-Thompson F, Alexander ME, Triedman JK, Gauvreau K, Walsh EP, Berul CI. Cardiac resynchronization therapy (and multisite pacing) in pediatrics and congenital heart disease: five years experience in a single institution. J Cardiovasc Electrophysiol 2009; 20(1):58-65.

24. Auricchio A. Pacing the left ventricle: does underlying rhythm matter? J Am Coll Cardiol 2004; 43(2):239-240.

25. Etienne Y, Mansourati J, Gilard M, Valls-Bertault V, Boschat J, Benditt DG, Lurie KG, Blanc JJ. Evaluation of left ventricular based pacing in patients with congestive heart failure and atrial fibrillation. Am J Cardiol 1999; 83(7):1138-1140, A1139. 
26. Touiza A, Etienne Y, Gilard M, Fatemi M, Mansourati J, Blanc JJ. Long-term left ventricular pacing: assessment and comparison with biventricular pacing in patients with severe congestive heart failure. J Am Coll Cardiol 2001; 38(7):1966-1970.

27. Vanagt WY, Verbeek XA, Delhaas T, Gewillig M, Mertens L, Wouters P, Meyns B, Daenen WJ, Prinzen FW. Acute hemodynamic benefit of left ventricular apex pacing in children. Ann Thorac Surg 2005; 79(3):932-936.

28. Tomaske M, Breithardt OA, Bauersfeld U. Preserved cardiac synchrony and function with singlesite left ventricular epicardial pacing during mid-term follow-up in paediatric patients. Europace 2009; 11(9):1168-1176.

29. Dodge-Khatami A, Kadner A, Dave H, Rahn M, Pretre R, Bauersfeld U. Left heart atrial and ventricular epicardial pacing through a left lateral thoracotomy in children: a safe approach with excellent functional and cosmetic results. Eur J Cardiothorac Surg 2005; 28(4):541-545.

30. McLeod KA. Cardiac pacing in infants and children. Heart 2010; 96(18):1502-1508.

31. Shalganov TN, Paprika D, Vatasescu R, Kardos A, Mihalcz A, Kornyei L, Szatmari A, Szili-Torok T. Mid-term echocardiographic follow up of left ventricular function with permanent right ventricular pacing in pediatric patients with and without structural heart disease. Cardiovasc Ultrasound 2007; 5:13.

32. van Geldorp IE, Vernooy K, Delhaas T, Prins MH, Crijns HJ, Prinzen FW, Dijkman B. Beneficial effects of biventricular pacing in chronically right ventricular paced patients with mild cardiomyopathy. Europace 2010; 12(2):223-229.

33. Vanagt WY, Prinzen FW, Delhaas T. Reversal of pacing-induced heart failure by left ventricular apical pacing. N Engl J Med 2007; 357(25):2637-2638.

34. Tomaske M, Breithardt OA, Balmer C, Bauersfeld U. Successful cardiac resynchronization with single-site left ventricular pacing in children. Int J Cardio/ 2009; 136(2):136-143. 


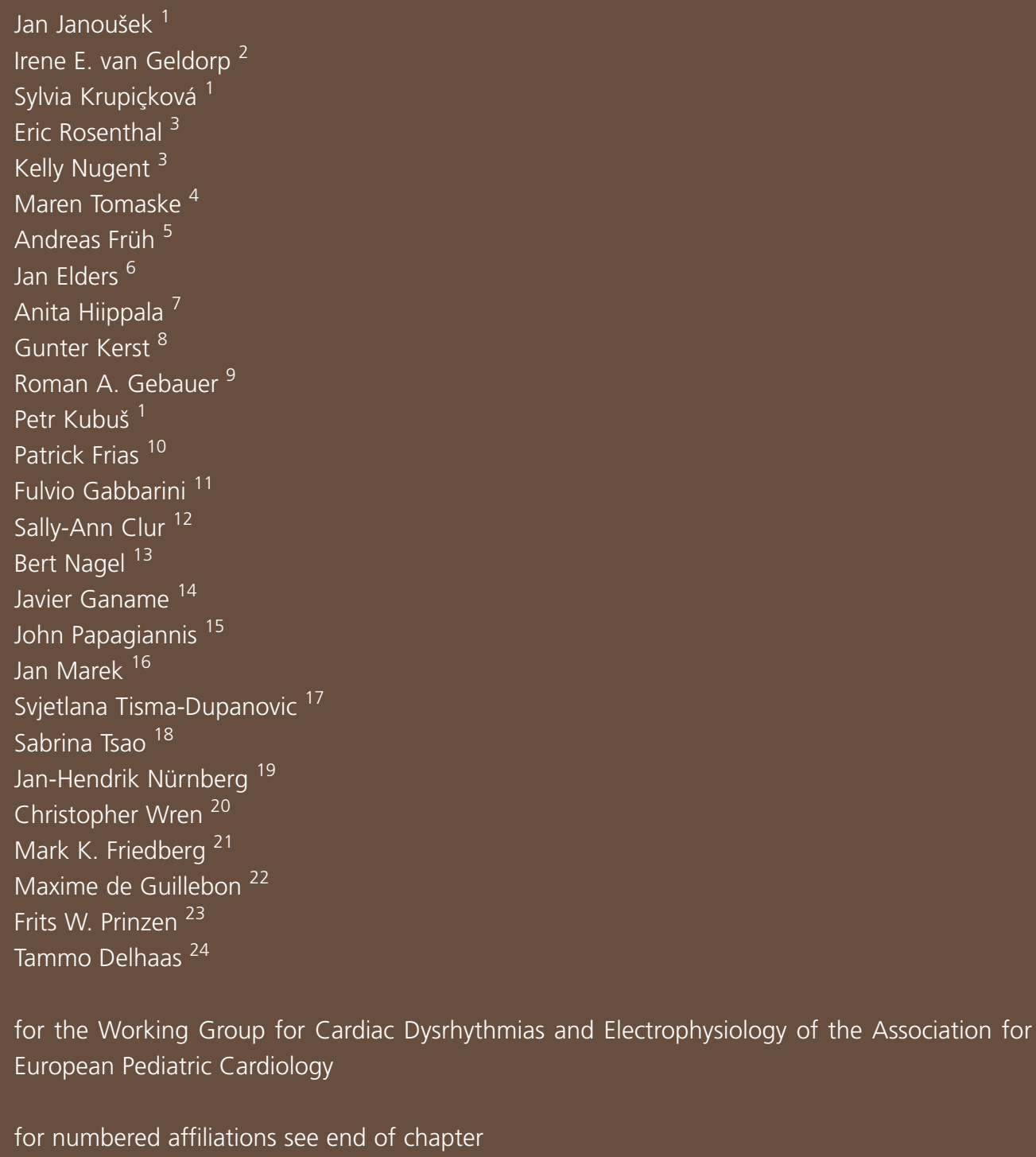



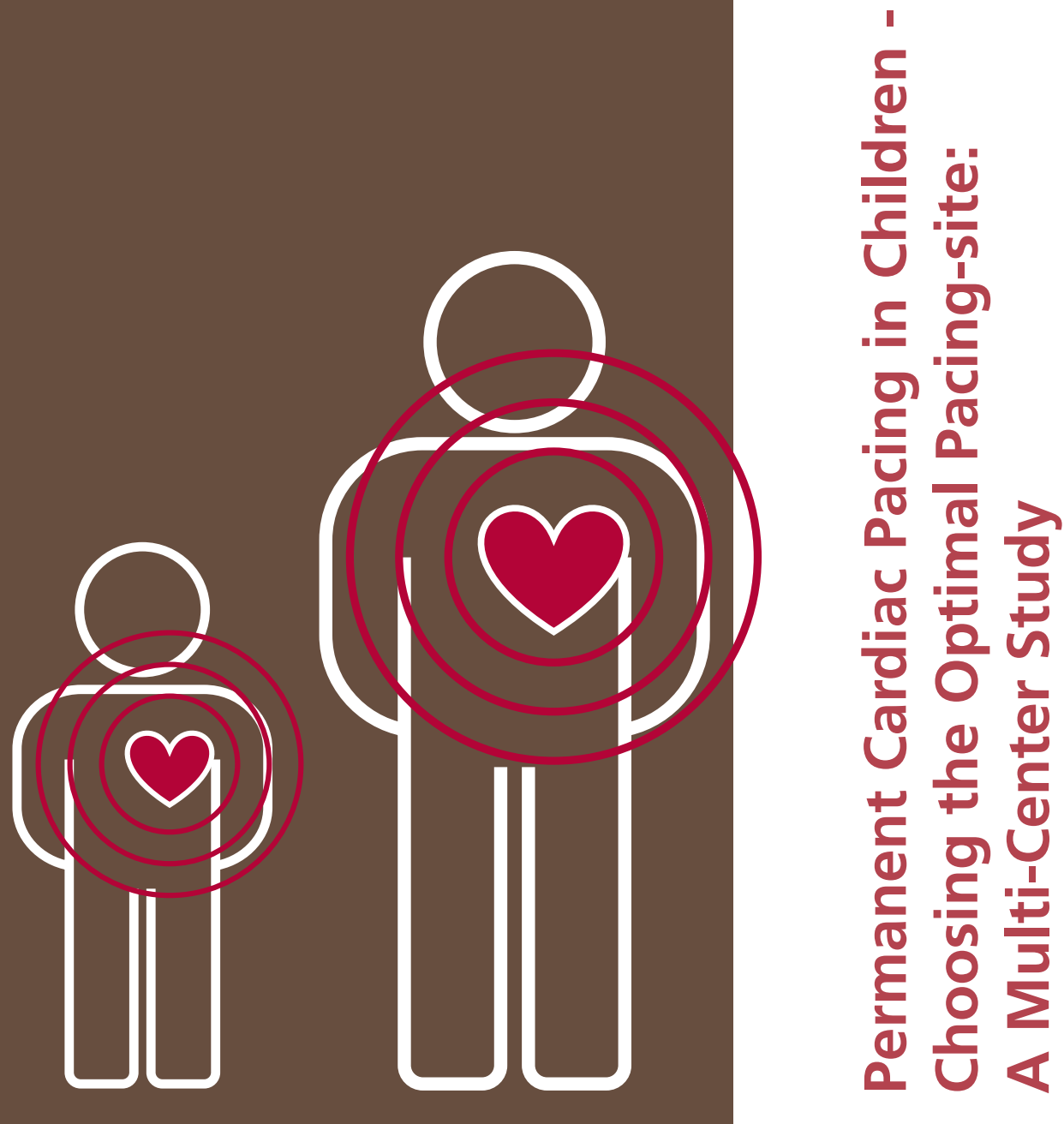


\section{Abstract}

Background: We evaluated the effects of the site of ventricular pacing on left ventricular (LV) synchrony and function in children requiring permanent pacing.

Methods: 178 children (age <18 years) from 21 centers with complete atrioventricular block and a structurally normal heart undergoing permanent pacing were cross-sectionally studied. Median age at evaluation was 11.2 (inter-quartile range (IQR) 6.3-15.0) years. Median pacing duration was 5.4 (IQR 3.1-8.8) years. Data were analyzed in a core lab. Pacing-sites were the free wall of the right ventricular (RV) outflow tract (RVOT, N=8), lateral RV (RVLat, N=44), RV apex (RVA, $N=61), R \bigvee$ septum (RVS, $N=29$ ), LV apex (LVA, $N=12$ ), LV mid-lateral wall (LVLat, $N=17$ ), and LV base (LVB, $N=7)$.

Results: LV synchrony (assessed by 2-dimensional strain), pump function (ejection fraction (EF), end-systolic volume index, shortening fraction) and contraction efficiency were significantly affected by pacing-site and were superior in children paced at LVA/LVLat. LV dyssynchrony correlated inversely with LV EF $(R=0.80, P=0.031)$. Pacing from RVOT/RVLat predicted decreased LV function (LV EF <45 \%; OR $5.19 \mathrm{Cl} 1.74-15.50, \mathrm{P}=0.003$ ) whereas LVA/LVLat pacing was associated with preserved LV function (LV EF >55 \%; OR 6.97, Cl 2.21-22.00, P<0.001). Age at implantation, pre-implantation LV size and function, duration of pacing, DDD mode, QRS duration, and presence of maternal auto-antibodies had no significant impact on LV EF in a multivariable analysis.

Conclusion: The site of ventricular pacing has a major impact on LV mechanical synchrony, efficiency and pump function in children that require life-long pacing. The preferred LVA/LVLat site can be reached using existing tools, allowing easy prevention of pacing-induced heart failure. 


\section{INTRODUCTION}

Right ventricular (RV) pacing has been used for decades in both adults and children. Recently, several large adult studies, ${ }^{1-3}$ smaller pediatric reports, ${ }^{4-7}$ and one larger pediatric survey ${ }^{8}$ have pointed towards the adverse effects of RV pacing. The incidence of left ventricular (LV) dysfunction in RV paced children ranged within a medium follow-up of less than a decade from 6.0 to $13.4 \% .4$ The impact of pacing-induced dyssynchrony may be especially important in children with a prospect of life-long pacing that lasts for decades. This idea is nourished by findings that dyssynchronous LV activation causes pathologic remodeling and dysfunction. ${ }^{9}$ Pediatric pacemaker therapy represents an optimal model for the evaluation of the long-term effects of different pacing-sites because, based on surgical preferences and in contrast to adults, various pacing-sites are used including LV epicardial pacing. In small single center reports ${ }^{10-14}$ and one larger retrospective survey ${ }^{8}$ pacing from the LV apex or free wall was associated with better preservation of LV function. The purpose of the current multi-center study was to evaluate the influence of different ventricular pacing-sites on long-term LV function in children with nonsurgical atrioventricular block and a structurally normal heart, and to search for a mechanism for the difference in pump function between sites, by measuring mechanical synchrony and efficiency in a cross-sectional echocardiographic evaluation.

\section{Patients AND Methods}

\section{Recruitment and demography}

Patients were recruited from 21 centers providing pacemaker therapy for children (17 European and four North American). According to the study protocol (available online) the patients had to fulfill the following inclusion criteria: presence of second or third degree atrioventricular (AV) block necessitating permanent cardiac pacing with $>70 \%$ ventricular paced beats, age $<18$ years at the time of primary pacemaker implantation, absence of any but trivial structural heart disease and of any known systemic illness potentially influencing cardiac function, duration of pacing more than one year, and no change in the ventricular pacing-site during the follow-up period. A total of 178 patients (female: 96, male: 82) were included in the study with a median age at pacemaker implantation of 3.2 , inter-quartile range (IQR) $0.2-7.0$ years. AV block was congenital in 138 patients and diagnosed later during childhood in the remaining 40. Maternal auto-antibodies were present in 64 of the 136 mothers tested. Nine of the 178 patients had patent ductus arteriosus which was closed interventionally using coils prior to $(\mathrm{N}=3)$, at the time of $(\mathrm{N}=3)$, or after $(\mathrm{N}=3)$ pacemaker implantation. The retrospectively gathered data further included demographic parameters, preimplantation LV size and function, New York Heart Association (NYHA) classification, and pacemaker implantation details (pacing-site as recorded by the implanting physician, lead type: endocardial vs epicardial, and initial pacing-mode and its change during the follow-up period). 


\section{Cross-sectional evaluation}

After obtaining ethical approval by the hospital review committee and patient consent according to individual institutional guidelines, eligible patients were evaluated according to a pre-specified protocol including NYHA class assignment, 12-lead electrocardiogram (ECG), echocardiography, and, if not available in the patient files, a chest $x$-ray in the antero-posterior and lateral projections. The echocardiographic protocol consisted of: 1. two-dimensional grey scale loops of the parasternal long axis view, parasternal short axis view (at the level of papillary muscles), apical four-chamber view, and apical two-chamber view. 2. Parasternal long axis and short axis M-mode. 3. Pulsed Doppler of the RV- and LV-outflow tracts, pulsed transmitral Doppler, and qualitative assessment of mitral regurgitation (none $=0$, mild $=1$, moderate $=2$ and severe $=3$ ).

\section{Data analysis}

All data were analyzed in a core lab (Children's Heart Center, Prague, Czech Republic): 1. QRS duration was measured manually as the maximum value in any lead from ECG printouts with a sweep speed of 25 or $50 \mathrm{~mm} / \mathrm{s}$; 2. Pacing-site assignment was performed using 12-lead ECG QRS morphology and axis ${ }^{15}$ and bi-plane chest $x$-rays from either the cross-sectional evaluation or any time before; pacing-sites were grouped into seven categories for the purpose of statistical evaluation: free wall of the RV outflow tract (RVOT), lateral RV wall (RVLat), RV apex (RVA), RV septum (RVS) (any position), LV apex (LVA), lateral LV wall (LVLat), and LV base (LVB). Details of the echocardiographic analysis are available on-line.

\section{Statistics}

Data are presented as mean (standard deviation) or as median (25-75\% inter-quartile range) according to their distribution. Multiple comparisons between different patient groups were performed by one way analysis of variance followed by pair-wise multiple comparisons using the Holm-Sidak method for normally distributed data. The Kruskal-Wallis one way analysis of variance on ranks followed by pair-wise multiple comparisons by the Dunn's method was used in the absence of normal distribution. Analysis of covariance was further used to elucidate the influence of covariates on specific patient group data. For comparisons of categorical variables the chi-square test was applied. Correlation between two variables was evaluated by linear regression. Inter-observer variability of two-dimensional strain measurements was tested by the coefficient of variation. ${ }^{16}$ Independent variables showing univariate differences $(P<0.2)$ related to decreased LV function ( $E F<50 \%$ ) were entered into the multiple logistic regression analysis. SigmaPlot for Windows Version 9.0 (Systat Software Inc., San Jose, California, USA) and PASW Statistics 18.0.3 (IBM Corporation, New York, USA) were used for statistical analysis. Significance was accepted at the $\mathrm{P}<0.05$ level. 


\section{RESULTS}

Cross-sectional evaluation was performed at a median age of 11.2 (IQR 6.3-15.0) years. Median pacing duration was 5.4 (IQR 3.1-8.8) years.

\section{Pacing-sites}

In total, 97 patients were paced epicardially and the remaining 81 from the endocardium. Patients were not distributed equally with respect to pacing-site, reflecting the historical preference for RV pacing. Demographic and clinical parameters for the respective pacing-sites are summarized in Table 1. Patients paced from the LVA were younger and had a shorter followup and QRS duration than those paced from the various RV sites. Also, gender distribution and the proportion of DDD paced patients were not equal.

\section{Left ventricular function}

Left ventricular shortening fraction, ejection fraction and the end-systolic volume index were different between pacing-sites, whereas the z-score of the LV end-diastolic dimension and the end-diastolic volume index did not differ. LVA and LVLat pacing yielded significantly higher shortening fraction and EF, and lower end-systolic volume index than RV pacing-sites (Figure 1). LV EF was not significantly different between RVS and RVA pacing. Patients with RVOT and RVLat pacing had the largest scatter in LV EF with the lower quartile as low as $<38 \%$ in the RVOT group (Figure 1B). Patients with LV EF $<50 \%$ were confined to RV pacing-sites whereas all patients paced from the LV had preserved LV function (Figure 2). As compared to the preimplantation values the decrease in LV shortening fraction was significant for all RV pacing-sites (median -7.8, IQR -12.3 to -2.5 SF units, P <0.001) and absent in the LV paced groups (median 0.3, IQR -6.7 to $+4.1 \mathrm{SF}$ units, NS, Figure 3). To elucidate the potential effect of maternal autoantibodies on LV function in the respective pacing-site groups, analysis of covariance was used. Pacing-site was still the most significant predictor of both LV EF and shortening fraction ( $P$ $<0.001$ for both) whereas antibody status reached marginal significance only for LV EF ( $P$ $=0.033)$. RVOT/RVLat pacing was the only significant independent predictor of decreased LV EF $(<45 \%)$ whereas LVA/LVLat pacing was associated with preservation of LV function (LV EF >55 $\%$, Table 2 and 3). Duration of pacing did not show any significant influence on LV function in the univariate and multi-variable analysis (Table 2 and 3). QRS duration did not correlate with either the LV EF or the decrease in LV shortening fraction as compared to pre-implantation values in the whole cohort, or if evaluated separately in the RV and LV paced subgroups, respectively. To allow for comparison with a recent multicenter retrospective survey, ${ }^{8}$ we also analyzed LV function by whether they were RV epicardial, RV endocardial or LV paced. Results were similar to the previous findings ${ }^{8}$ with LV pacing being superior to RV endocardial or epicardial pacing in terms of LV shortening fraction, LV EF, and change in LV shortening fraction as compared to pre- 


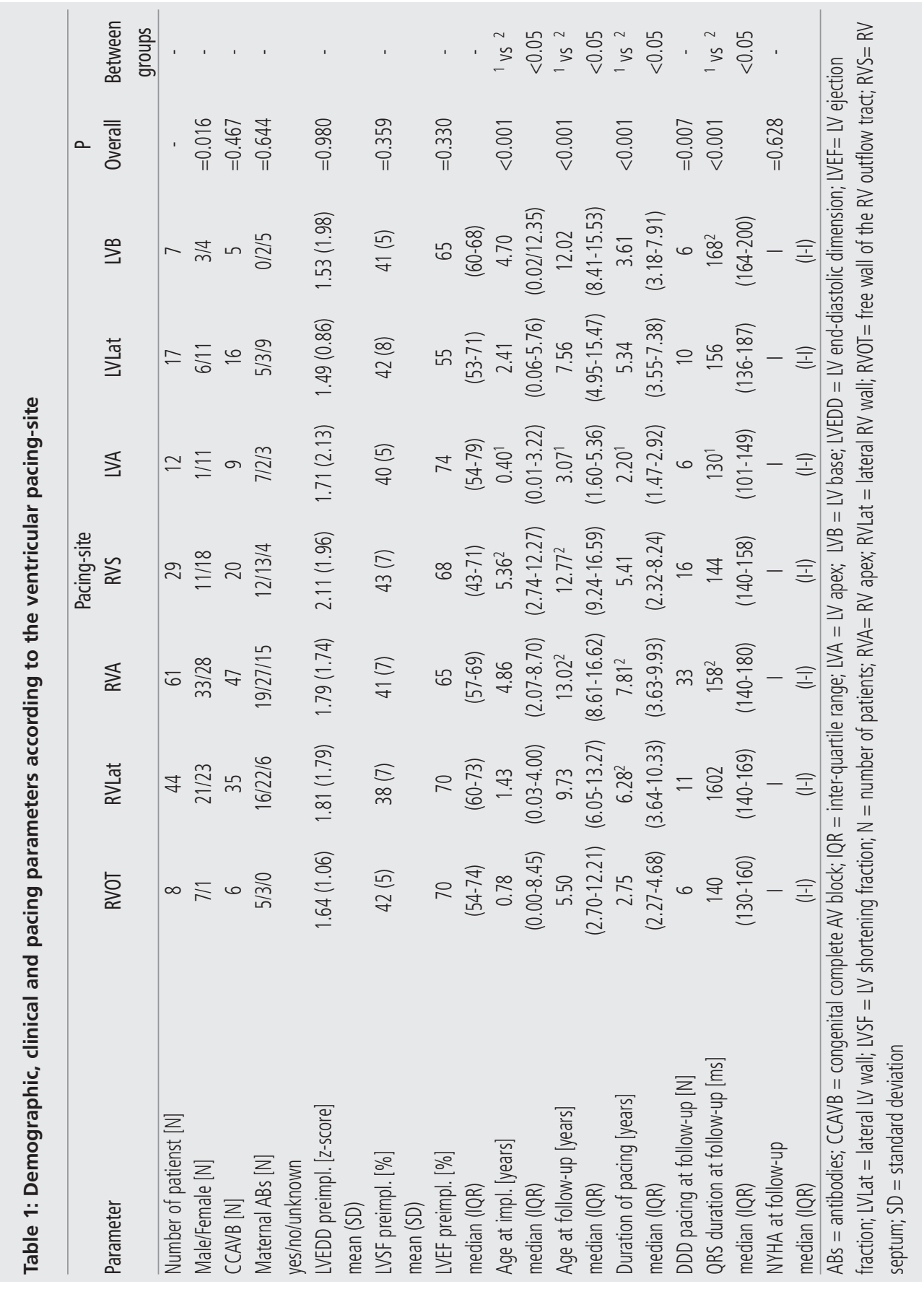


A

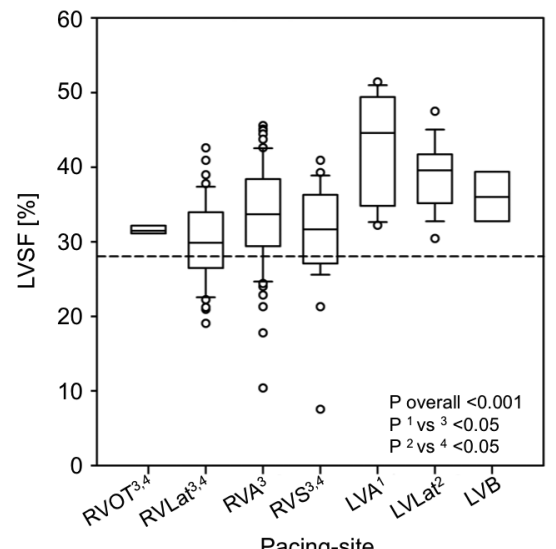

B

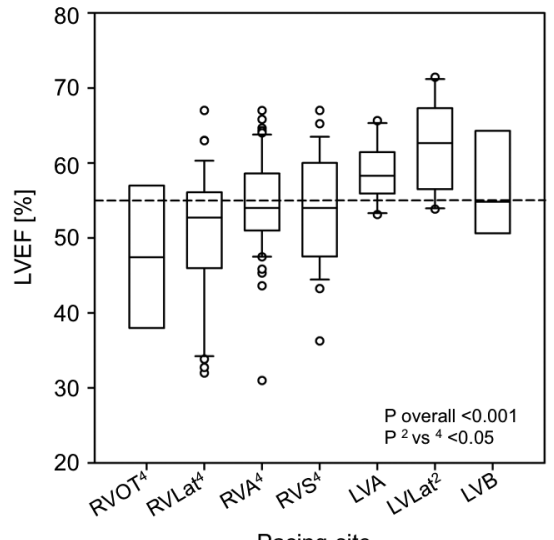

C

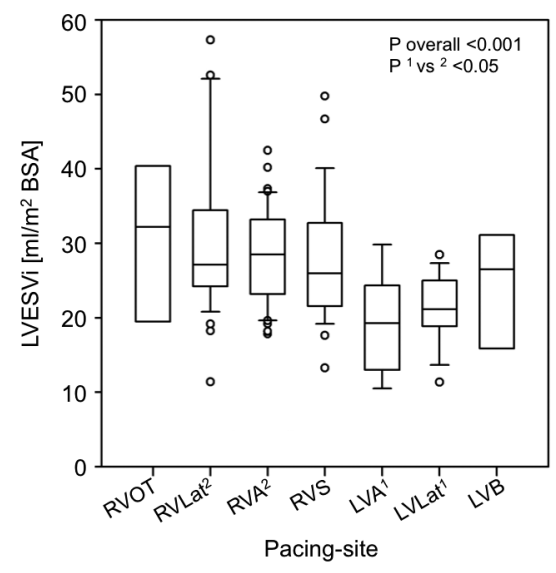

Figure 1. LV function at cross-sectional follow-up.

A. LV shortening fraction. B. LV ejection fraction. C. LV end-systolic volume index. The dashed line shows the division between normal and subnormal values. LVA = LV apex; LVB = LV base; LVLat = lateral LV wall; $\mathrm{LV} E F=\mathrm{LV}$ ejection fraction; LVESVi = LV end-systolic volume index; LV SF = LV shortening fraction; RVA= $R V$ apex; RVLat = lateral RV wall; RVOT = free wall of the RV outflow tract; RVS = RV septum

implantation values ( $P<0.05$ for all, Table 4). No difference was found between RV epicardial and endocardial pacing in this analysis. 


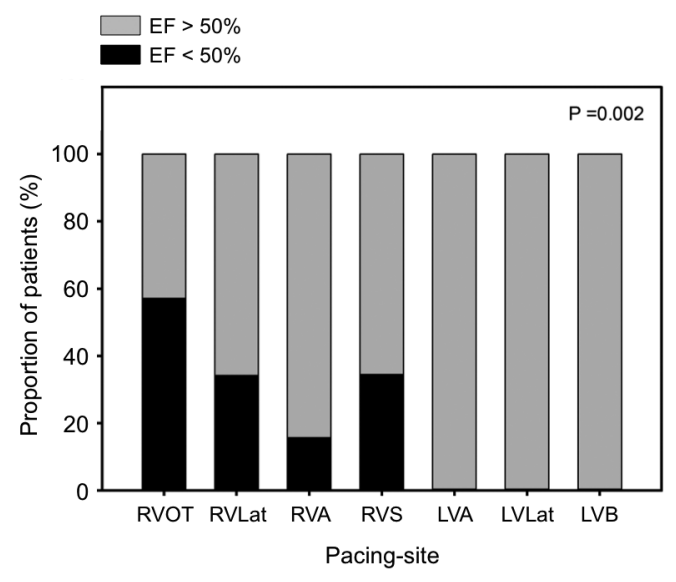

Figure 2. Proportion of patients with decreased left ventricular ejection fraction.

$\mathrm{EF}=$ ejection fraction; for other abbreviations see Figure 1.

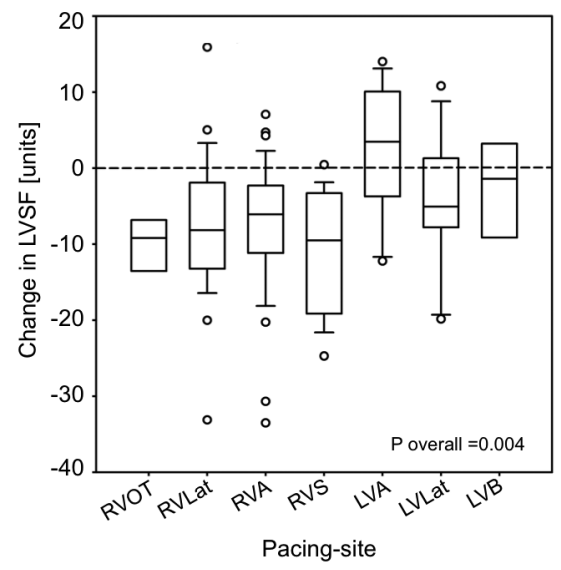

Figure 3: Change in LV shortening fraction from pre-implantation to cross-sectional follow-up.

Dashed line $=$ no change. Abbreviations see Figure 1 .

\section{Left ventricular dyssynchrony}

The inter-ventricular and intra-LV delays were significantly different between pacing-sites (Figure 4). Segmental strain analysis by speckle tracking confirmed this mechanical dyssynchrony pattern (Figure 5 and 6A, B). RV pacing consistently produced delayed LV ejection and a mechanical contraction delay between the septum and LV free wall with least negative effect of the RVA 
Table 2: Risk factors for decreased LV function (LV EF <45 \%)

\begin{tabular}{lccccc}
\multicolumn{5}{c}{ Univariate } & Multi-variable \\
\hline Variable & LV EF $<45 \%$ & LV EF $>45 \%$ & $P=$ & $P=$ & OR (5-95\% Cl) \\
Male gender & $50.0 \%$ & $45.4 \%$ & 0.931 & - & - \\
Congenital AV block & $75.0 \%$ & $77.5 \%$ & 0.932 & - & - \\
Maternal autoantibodies & $61.5 \%$ & $43.6 \%$ & 0.458 & - & - \\
Age at implantation [years, median] & 1.80 & 3.18 & 0.837 & - & - \\
RVOT and RVLat pacing & $62.5 \%$ & $24.8 \%$ & 0.004 & 0.003 & $5.19(1.74-15.50)$ \\
DDD pacing & $50.0 \%$ & $48.2 \%$ & 0.897 & - & - \\
Pacing duration [years, median] & 4.40 & 5.39 & 0.175 & 0.154 & $0.89(0.75-1.05)$ \\
QRS duration [ms, median] & 145 & 154 & 0.689 & - & -
\end{tabular}

Abbreviations see Table 1.

Table 3: Factors associated with preservation of LV function (LV EF >55 \%)

\begin{tabular}{lccccc}
\multicolumn{7}{c}{ Univariate } & Multi-variable & & & \\
\hline Variable & LV EF $>55 \%$ & LV EF $<55 \%$ & $P=$ & $P=$ & OR (5-95 \% CI) \\
Male gender & $36.1 \%$ & $54.1 \%$ & 0.036 & 0.237 & $0.66(0.33-1.32)$ \\
Congenital AV block & $73.6 \%$ & $80.5 \%$ & 0.410 & - & - \\
Maternal autoantibodies & $41.1 \%$ & $49.3 \%$ & 0.391 & - & - \\
Age at implantation [years, median] & 3.22 & 2.89 & 0.598 & - & - \\
LVA and LVLat pacing & $4.7 \%$ & $29.2 \%$ & $<0.001$ & $<0.001$ & $6.97(2.21-22.00)$ \\
DDD pacing & $45.8 \%$ & $50.6 \%$ & 0.664 & - & - \\
Pacing duration [years, median] & 5.02 & 5.70 & 0.304 & - & - \\
QRS duration [ms, median] & 150 & 157 & 0.042 & 0.080 & $0.986(0.97-1.00)$
\end{tabular}

Abbreviations see Table 1.

pacing-site. In contrast, during LVA and LVLat pacing both inter- and intra- ventricular dyssynchrony were minimal. Pacing-sites located towards the LVB resulted in a reversed intra-LV dyssynchrony pattern with early free wall and late septal motion. LV EF was significantly dependent on the degree of LV dyssynchrony (Figure 6C). 
Table 4: Differences between RV epicardial, endocardial and LV pacing

\begin{tabular}{lccccc} 
& \multicolumn{3}{c}{ Pacing-site } & P \\
& $\begin{array}{c}\text { RV } \\
\text { epicardial } \\
\text { pacing }\end{array}$ & $\begin{array}{c}\text { RV } \\
\text { endocardial } \\
\text { pacing }\end{array}$ & $\begin{array}{c}\text { LV } \\
\text { pacing }\end{array}$ & Overall & $\begin{array}{c}\text { Between } \\
\text { groups }\end{array}$ \\
\hline LVSF [\%] & 31 & 33 & 39 & $<0.001$ & ${ }^{3}$ vs $^{1,2}$ \\
median (IQR) & $(27-35)$ & $(29-37)$ & $(35-44)$ & & $<0.05$ \\
Change in LVSF [SF units] & -9 & -6 & 0 & $=0.001$ & ${ }^{3}$ vs $^{1,2}$ \\
median (IQR) & $(-13--3)$ & $(-13--2)$ & $(-7-4)$ & & $<0.05$ \\
LVEF [\%] & 53 & 53 & 60 & $<0.001$ & ${ }^{3}$ vs $^{1,2}$ \\
median (IQR) & $(49-58)$ & $(48-57)$ & $(55-65)$ & & $<0.05$ \\
\hline
\end{tabular}

A

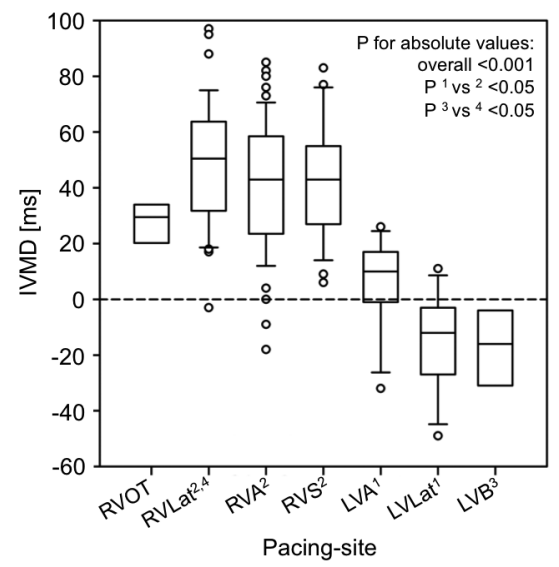

B

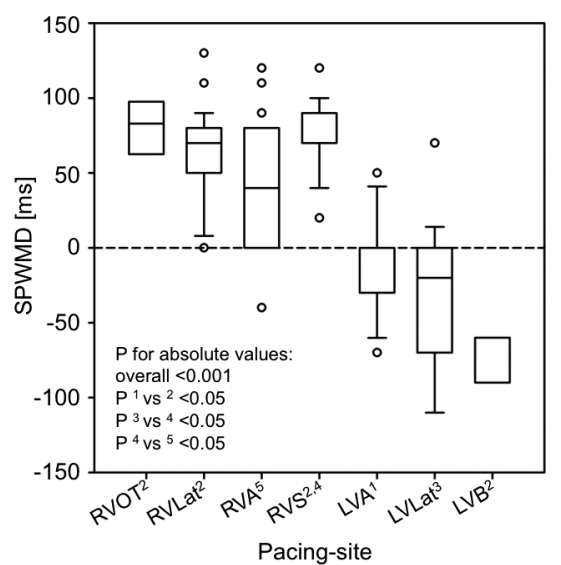

Figure 4:

A. Inter-ventricular mechanical delay. B. Septal to posterior wall motion delay. To allow for comparisons between pacing sites statistical significance is calculated for absolute measurement values. Dashed line $=$ inter-/intraventricular synchrony. IVMD = inter-ventricular mechanical delay; SPWMD = septal to posterior wall motion delay; for other abbreviations see Figure 1.

\section{Contraction efficiency}

The proportion of wasted LV contraction due to dyssynchrony (see details of the echocardiographic analysis as available on-line) was significantly higher during RV pacing than during LV pacing for both radial and longitudinal systolic function: median 8.3 (IQR 5.7-14.5) vs $3.1(2.2-3.5), P=0.002$ and $6.2($ IQR 5.0-8.2) vs $2.1(1.2-3.5) \%, P<0.001$, respectively. 

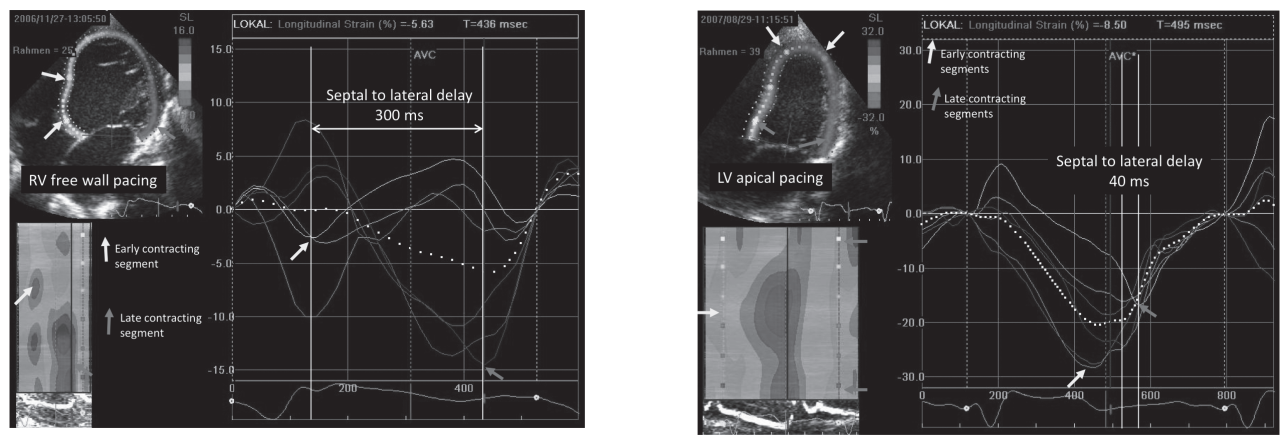

\section{Figure 5:}

A. Mechanical activation pattern in RV free wall pacing showing early peak negative 2D strain in the basal and mid-ventricular septum (white arrow) and late negative strain peak in the LV free wall (grey arrow). An extensive septal to lateral mechanical dyssynchrony with a delay of $300 \mathrm{~ms}$ is present. B. Left ventricular apical pacing with mechanical activation starting at the apex (white arrows) and proceeding to the base (grey arrows) resulting in almost complete septal to lateral mechanical synchrony.

A

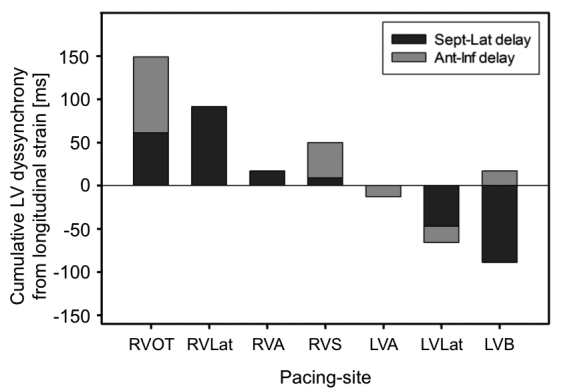

Figure 6:

A. Cumulative LV dyssynchrony A. using longitudinal strain in the apical four- and two-chamber views and B. using radial strain in the parasternal short axis view. $C$. Relationship between the degree of cumulative LV dyssynchrony using radial strain and LV ejection fraction. Ant-Inf delay = anterior to inferior delay; As-Post delay = anteroseptal to posterior delay; Sept-Lat delay = septal to lateral delay; other abbreviations see Figure 1.
B

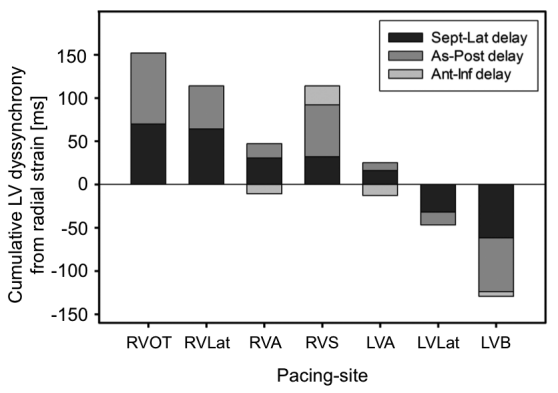

C

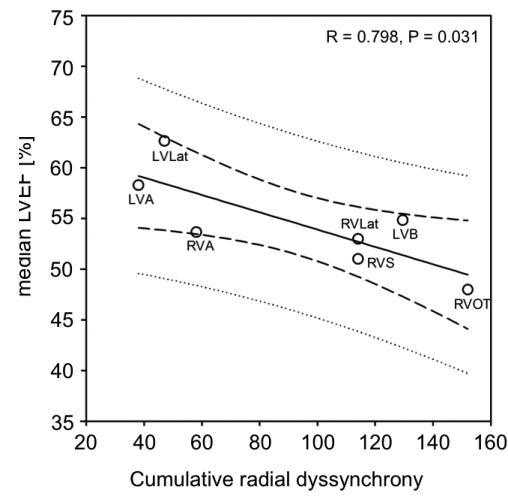




\section{Inter-observer agreement}

Inter-observer agreement ( $\mathrm{J}, \mathrm{IVG}$ ) was calculated in a total of 28/178 patients. Pacing-site assignment was equal in 27/28 patients. The following coefficients of variation were achieved in the parameters tested: biplane LV ejection fraction $(\mathrm{EF})=9.7 \%$, inter-ventricular mechanical delay $=5.7 \%$, septal to posterior wall motion delay $=11.2 \%$, inter-segmental mechanical delay from two-dimensional strain $=0.9 \%$.

\section{Discussion}

This is the first cross-sectional multi-center study showing significant differences between various ventricular pacing-sites in terms of LV synchrony, function and contraction efficiency in a large group of children that are chronically paced for complete AV block in the absence of structural heart disease. The results can be summarized as follows:

1. LV apical and LV lateral wall pacing are associated with the best preservation of LV function, which appears related to preserved mechanical synchrony and contraction efficiency.

2. RV pacing-sites carry a high risk for a negative effect on LV performance, coinciding with significant mechanical asynchrony and contraction inefficiency. This effect is most pronounced for RV lateral and outflow tract pacing and less with RV apical pacing.

3. Non-targeted RV septal pacing does not show any advantage over RV apical pacing.

4. LV basal pacing produces a significant reversed pattern of LV dyssynchrony and should probably not be the preferred LV pacing-site.

5. The presence of maternal auto-antibodies is not consistently associated with decreased LV function and is only a marginal modifier of the response to pacing-induced LV dyssynchrony. These results strongly support previous findings of a retrospective pediatric study ${ }^{4}$ showing a decrease in LV function specifically due to RV free wall pacing. Our results also confirm data on preservation of LV function with LV apical or LV lateral wall pacing ${ }^{10-14}$ including a large retrospective pediatric multi-center survey published recently. ${ }^{8}$ This report does not show any superiority of RV septal over RV apical pacing. This is in line with an elegant experimental study recently published. ${ }^{17}$ Some studies showed promising results using RV septal lead placement, ${ }^{18}$ but clear benefit from RV septal pacing has not yet been demonstrated in a randomized study, except when the lead is positioned in the His-bundle. ${ }^{19}$ RV pacing (contrary to LV pacing) was associated with depressed systolic function and induced a consistent decrease in LV systolic function compared to pre-implantation values. This decrease was functionally well tolerated as no difference in the NYHA class was observed between the pacing-sites. However, given the cross-sectional design of the study, patients suffering from symptomatic heart failure may have been missed because they were upgraded to a biventricular system, were transplanted or died. The incidence of patients suffering from overt heart failure due to RV pacing has been reported to range from 6.0 to $13.4 \%$ in previous pediatric reports. $4,6,7$ 
Results of this study further indicate that LV pacing may be a substitute for primary biventricular pacing which has recently been shown to preserve LV function in chronically paced adults. ${ }^{20} \mathrm{As}$ demonstrated by Tomaske et al. and Vanagt et al. in small descriptive pediatric reports, ${ }^{21,22} \mathrm{LV}$ pacing may also be used instead of biventricular pacing to improve LV function which has been compromised from long-term RV pacing.

QRS duration was not a multivariable predictor of decreased LV function as it reflects the total electrical activation time but not the sequence of activation. Recently, a sub-analysis of the MADIT-CRT trial has shown that left bundle branch block morphology rather than QRS duration is the prerequisite for the efficacy of cardiac resynchronization therapy. ${ }^{23}$ This implies that a specific activation pattern is more important than total asynchrony. Our study indicates that the negative effects of LV dyssynchrony produced by RV pacing, are preventable by LV pacing irrespective of QRS duration.

The presence of maternal auto-antibodies in the setting of congenital AV block was not found to be a strong component of the individual reactivity to the pacing-induced LV dyssynchrony as opposed to a study showing association of autoimmune AV block with dilated cardiomyopathy. ${ }^{24}$ None of the patients who were paced from the LV showed decreased LV function, despite the presence of maternal auto-antibodies in a significant portion. RV pacinginduced LV dysfunction has previously been reported in the absence of maternal auto-antibodies in children with surgical AV block and could be effectively corrected by an upgrade to biventricular pacing. ${ }^{25,26}$ All these findings support our statement that the pacing-site plays a crucial role in the development of pacing-associated LV dysfunction.

\section{Limitations}

This study has limitations related to the unequal number of patients in each pacing-site group, significant differences in age at primary implantation and duration of pacing, as well as the accuracy of the retrospective assessment of the pacing-site using surgical records, biplane x-ray and 12-lead ECG. However, neither age nor duration of pacing was a multi-variable predictor of LV dysfunction in this study. Pacing-site localization could be performed with acceptable interobserver variability. Thus, we do not feel that these limitations significantly skew the results.

\section{Clinical implications and context}

Permanent cardiac pacing that starts in childhood will continue for decades. In the current study mean age was 11 and mean pacing duration 5.5 years, so the observed reduction in LV function in RV-paced children is only the beginning of a process that will likely develop further over subsequent decades. ${ }^{9,} 27$ Thus, the aim to preserve LV synchrony and function should be mandatory despite a degree of unpredictable individual variability in the response to electromechanical dyssynchrony. Patients with a systemic LV who are scheduled for epicardial lead implantation should be paced from the LV apex or free wall, whereas the RV free wall and outflow 
tract should be avoided. Transvenous leads may be placed in the RV apex given the fact, that it had the least negative hemodynamic influence of all RV pacing-sites. These principles should be applied to all children with a systemic LV and either spontaneous or surgical AV block. Surgical access to the LV is possible using existing tools and at no additional costs: the subxiphoid approach in younger children or, in older ones, a left lateral thoracotomy with an excellent cosmetic result. ${ }^{28}$ Care should be taken to place the leads rather at the LV apex than the LV base, as the inverse pattern of electromechanical dyssynchrony caused by LV basal pacing might be detrimental in the long term. The results of the present study provide an important clinical confirmation of previously published experimental research conducted and summarized by the coauthors of this report. ${ }^{27,29}$ The results of this study may also have importance for future strategy of pacemaker therapy in adults given the fast developments in pacemaker technology and the expected introduction of leadless pacing systems in the near future with a potential for an easy application of LV pacing.

\section{Conclusion}

Pacing-induced deterioration of LV function is easily preventable by pacing the LV apical or midlateral wall. On the other hand RV free wall and outflow tract pacing carry the highest risk of pacing-induced LV dysfunction and should be avoided. Added to the previously published experimental and clinical data on the influence of ventricular pacing-sites on LV mechanics and function this large study will have significant implications on pacemaker therapy in children.

\section{Funding}

JJ, PK and SK were supported by a research grant of the Internal Grant Agency of the Ministry of Health of the Czech Republic (JJ, PK, and SK: NT 12321-3/2011); IEvG was supported by a Dr. E. Dekker Grant for Research Fellow in Pediatric Cardiology, Dutch Heart Foundation, NHS$2010 T 078$. 


\section{Author affiliations}

${ }^{1}$ Children's Heart Centre, University Hospital Motol, Prague, Czech Republic; ${ }^{2}$ Pediatric Cardiology, Cardiovascular Research Institute Maastricht, Maastricht University Medical Center, Maastricht, The Netherlands; ${ }^{3}$ Evelina Children's Hospital, London, United Kingdom; ${ }^{4}$ University Children's Hospital, Zurich, Switzerland; ${ }^{5}$ Oslo University Hospital, Oslo, Norway; ${ }^{6}$ Dept of Cardiology, UMC St. Radboud, Nijmegen, The Netherlands; ${ }^{7}$ Department of Pediatric Cardiology, Children's Hospital, University of Helsinki and Helsinki University Central Hospital; ${ }^{8}$ Pädiatrische Kardiologie, Universitätsklinik für Kinder- und Jugendmedizin, Tübingen, Germany; ${ }^{9}$ Department of Pediatric Cardiology, University of Leipzig, Heart Centre, Leipzig, Germany; ${ }^{10}$ Department of Pediatric Cardiology, Children's Hospital of Atlanta, Atlanta, USA; ${ }^{11}$ Pediatric Cardiology Division, Children's Hospital Regina Margherita, Turin, Italy; ${ }^{12}$ Emma Children's Hospital - Academic Medical Center, Amsterdam, and Center for Congenital Heart Anomalies Amsterdam-Leiden, The Netherlands; ${ }^{13}$ Division of Pediatric Cardiology, Children's Hospital, Medical University Graz, Austria; ${ }^{14}$ Pediatric Cardiology, University Hospital Leuven, Leuven, Belgium; ${ }^{15}$ Division of Pediatric Cardiology, Mitera Children's Hospital, Maroussi, Greece; ${ }^{16}$ Pediatric Cardiology, Great Ormond Street Hospital, London, United Kingdom; ${ }^{17}$ Cardiology Section, Children's Mercy Hospitals and Clinics, Kansas City, USA; ${ }^{18}$ Division of Cardiology, Children's Memorial Hospital, Chicago, USA; ${ }^{19}$ Klinikum Links der Weser, Abt. Pediatric Cardiology, Bremen, Germany; ${ }^{20}$ Pediatric Cardiology, The Newcastle upon Tyne Hospitals, NHS Foundation Trust, Newcastle upon Tyne, United Kingdom; ${ }^{21}$ Division of Cardiology, The Hospital for Sick Children, Toronto, ON, Canada; ${ }^{22}$ Department of Congenital Heart Disease, Hôpital Cardiologique du Haut-Lévêque, Bordeaux University Hospitals, Bordeaux-Pessac, France; ${ }^{23}$ Department of Physiology, Cardiovascular Research Institute Maastricht, Maastricht University, Maastricht, The Netherlands; ${ }^{24}$ Department of Biomedical Engineering, Cardiovascular Research Institute Maastricht, Maastricht University, Maastricht, The Netherlands 
Chapter 7 


\section{REFERENCES}

1. Sweeney MO, Hellkamp AS, Ellenbogen KA, Greenspon AJ, Freedman RA, Lee KL, Lamas GA. Adverse effect of ventricular pacing on heart failure and atrial fibrillation among patients with normal baseline QRS duration in a clinical trial of pacemaker therapy for sinus node dysfunction. Circulation 2003; 107(23):2932-2937.

2. Nielsen JC, Kristensen L, Andersen HR, Mortensen PT, Pedersen OL, Pedersen AK. A randomized comparison of atrial and dual-chamber pacing in 177 consecutive patients with sick sinus syndrome: echocardiographic and clinical outcome. J Am Coll Cardiol 2003; 42(4):614-623.

3. Wilkoff BL, Cook JR, Epstein AE, Greene HL, Hallstrom AP, Hsia H, Kutalek SP, Sharma A. Dualchamber pacing or ventricular backup pacing in patients with an implantable defibrillator: the Dual Chamber and VVI Implantable Defibrillator (DAVID) Trial. JAMA 2002; 288(24):3115-3123.

4. Gebauer RA, Tomek V, Salameh A, Marek J, Chaloupecky V, Gebauer R, Matejka T, Vojtovic P, Janousek J. Predictors of left ventricular remodelling and failure in right ventricular pacing in the young. Eur Heart J 2009; 30(9):1097-1104.

5. Karpawich PP, Rabah R, Haas JE. Altered cardiac histology following apical right ventricular pacing in patients with congenital atrioventricular block. Pacing Clin Electrophysiol 1999; 22(9):13721377.

6. Moak JP, Hasbani K, Ramwell C, Freedenberg V, Berger JT, DiRusso G, Callahan P. Dilated cardiomyopathy following right ventricular pacing for $\mathrm{AV}$ block in young patients: resolution after upgrading to biventricular pacing systems. J Cardiovasc Electrophysiol 2006; 17(10):1068-1071.

7. Kim JJ, Friedman RA, Eidem BW, Cannon BC, Arora G, Smith EO, Fenrich AL, Kertesz NJ. Ventricular function and long-term pacing in children with congenital complete atrioventricular block. J Cardiovasc Electrophysiol 2007; 18(4):373-377.

8. van Geldorp IE, Delhaas T, Gebauer RA, Frias P, Tomaske M, Friedberg MK, Tisma-Dupanovic S, Elders J, Fruh A, Gabbarini F, Kubus P, Illikova V, Tsao S, Blank AC, Hiippala A, Sluysmans T, Karpawich P, Clur SA, Ganame X, Collins KK, Dann G, Thambo JB, Trigo C, Nagel B, Papagiannis J, Rackowitz A, Marek J, Nurnberg JH, Vanagt WY, Prinzen FW, Janousek J. Impact of the permanent ventricular pacing site on left ventricular function in children: a retrospective multicentre survey. Heart 2011; 97(24):2051-2055.

9. Thambo JB, Bordachar P, Garrigue S, Lafitte S, Sanders P, Reuter S, Girardot R, Crepin D, Reant P, Roudaut R, Jais $P$, Haissaguerre M, Clementy J, Jimenez $M$. Detrimental ventricular remodeling in patients with congenital complete heart block and chronic right ventricular apical pacing. Circulation 2004; 110(25):3766-3772.

10. van Geldorp IE, Vanagt WY, Bauersfeld U, Tomaske M, Prinzen FW, Delhaas T. Chronic left ventricular pacing preserves left ventricular function in children. Pediatr Cardiol 2009; 30(2):125132. 
11. Vanagt WY, Verbeek XA, Delhaas T, Gewillig M, Mertens L, Wouters P, Meyns B, Daenen WJ, Prinzen FW. Acute hemodynamic benefit of left ventricular apex pacing in children. Ann Thorac Surg 2005; 79(3):932-936.

12. Vanagt WY, Verbeek XA, Delhaas T, Mertens $L$, Daenen WJ, Prinzen FW. The left ventricular apex is the optimal site for pediatric pacing: correlation with animal experience. Pacing Clin Electrophysiol 2004; 27(6 Pt 2):837-843.

13. Tomaske M, Breithardt OA, Bauersfeld U. Preserved cardiac synchrony and function with singlesite left ventricular epicardial pacing during mid-term follow-up in paediatric patients. Europace 2009; 11(9):1168-1176.

14. Gebauer RA, Tomek V, Kubus P, Razek V, Matejka T, Salameh A, Kostelka M, Janousek J. Differential effects of the site of permanent epicardial pacing on left ventricular synchrony and function in the young: implications for lead placement. Europace 2009; 11(12):1654-1659.

15. Kamakura S, Shimizu W, Matsuo K, Taguchi A, Suyama K, Kurita T, Aihara N, Ohe T, Shimomura $\mathrm{K}$. Localization of optimal ablation site of idiopathic ventricular tachycardia from right and left ventricular outflow tract by body surface ECG. Circulation 1998; 98(15):1525-1533.

16. Nilas L, Hassager C, Christiansen C. Long-term precision of dual photon absorptiometry in the lumbar spine in clinical settings. Bone Miner 1988; 3(4):305-315.

17. Mills RW, Cornelussen RN, Mulligan LJ, Strik M, Rademakers LM, Skadsberg ND, van Hunnik A, Kuiper M, Lampert A, Delhaas T, Prinzen FW. Left ventricular septal and left ventricular apical pacing chronically maintain cardiac contractile coordination, pump function and efficiency. Circ Arrhythm Electrophysiol 2009; 2(5):571-579.

18. Tse HF, Yu C, Wong KK, Tsang V, Leung YL, Ho WY, Lau CP. Functional abnormalities in patients with permanent right ventricular pacing: the effect of sites of electrical stimulation. J Am Coll Cardiol 2002; 40(8):1451-1458.

19. Zanon F, Bacchiega E, Rampin L, Aggio S, Baracca E, Pastore G, Marotta T, Corbucci G, Roncon L, Rubello D, Prinzen FW. Direct His bundle pacing preserves coronary perfusion compared with right ventricular apical pacing: a prospective, cross-over mid-term study. Europace 2008; 10(5):580-587.

20. Chan JY, Fang F, Zhang Q, Fung JW, Razali O, Azlan H, Lam KH, Chan HC, Yu CM. Biventricular pacing is superior to right ventricular pacing in bradycardia patients with preserved systolic function: 2-year results of the PACE trial. Eur Heart J 2011; 32(20):2533-2540.

21. Vanagt WY, Prinzen FW, Delhaas T. Reversal of pacing-induced heart failure by left ventricular apical pacing. N Engl J Med 2007; 357(25):2637-2638.

22. Tomaske M, Breithardt OA, Balmer C, Bauersfeld U. Successful cardiac resynchronization with single-site left ventricular pacing in children. Int J Cardiol 2009; 136(2):136-143. 
23. Zareba W, Klein H, Cygankiewicz I, Hall WJ, McNitt S, Brown M, Cannom D, Daubert JP, Eldar M, Gold MR, Goldberger JJ, Goldenberg I, Lichstein E, Pitschner H, Rashtian M, Solomon S, Viskin S, Wang P, Moss AJ. Effectiveness of Cardiac Resynchronization Therapy by QRS Morphology in the Multicenter Automatic Defibrillator Implantation Trial-Cardiac Resynchronization Therapy (MADITCRT). Circulation 2011; 123(10):1061-1072.

24. Villain E, Coastedoat-Chalumeau N, Marijon E, Boudjemline Y, Piette JC, Bonnet D. Presentation and prognosis of complete atrioventricular block in childhood, according to maternal antibody status. J Am Coll Cardiol 2006; 48(8):1682-1687.

25. Janousek J, Gebauer RA. Cardiac resynchronization therapy in pediatric and congenital heart disease. Pacing Clin Electrophysiol 2008; 31 Suppl 1:S21-23.

26. Janousek J, Gebauer RA, Abdul-Khaliq H, Turner M, Kornyei L, Grollmuss O, Rosenthal E, Villain E, Fruh A, Paul T, Blom NA, Happonen JM, Bauersfeld U, Jacobsen JR, van den Heuvel F, Delhaas T, Papagiannis J, Trigo C. Cardiac resynchronisation therapy in paediatric and congenital heart disease: differential effects in various anatomical and functional substrates. Heart 2009; 95(14):1165-1171.

27. Sweeney MO, Prinzen FW. Ventricular pump function and pacing: physiological and clinical integration. Circ Arrhythm Electrophysiol 2008; 1(2):127-139.

28. Dodge-Khatami A, Kadner A, Dave H, Rahn M, Pretre R, Bauersfeld U. Left heart atrial and ventricular epicardial pacing through a left lateral thoracotomy in children: a safe approach with excellent functional and cosmetic results. Eur J Cardiothorac Surg 2005; 28(4):541-545.

29. van Geldorp IE, Vanagt WY, Prinzen FW, Delhaas T. Chronic ventricular pacing in children: toward prevention of pacing-induced heart disease. Heart Fail Rev 2011; 16(3):305-314. 
Irene E. van Geldorp 1,2

Pierre Bordachar ${ }^{1}$

Joost Lumens 1,2

Maxime de Guillebon ${ }^{1}$

Zachary I. Whinnett ${ }^{1,3}$

Frits W. Prinzen ${ }^{2}$

Michel Haissaguerre ${ }^{1}$

Tammo Delhaas ${ }^{2}$

Jean-Benoît Thambo ${ }^{1}$

${ }^{1}$ Hôpital Cardiologique du Haut-Lévêque, Bordeaux University Hospital, Bordeaux, France;

${ }^{2}$ Cardiovascular Research Institute Maastricht, Maastricht University, Maastricht, The Netherlands; ${ }^{3}$ International Centre for Circulatory Health, National Heart and Lung institute, Imperial College London, United Kingdom

\section{Submitted 2012}




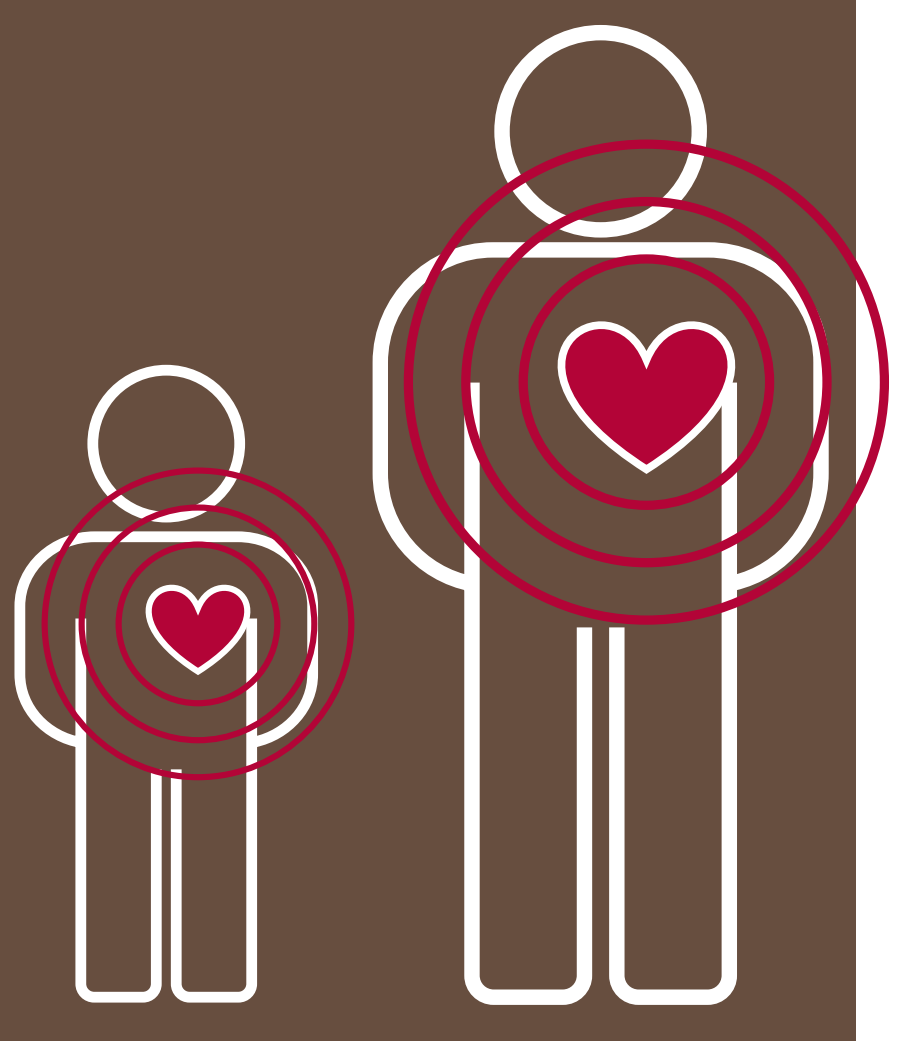

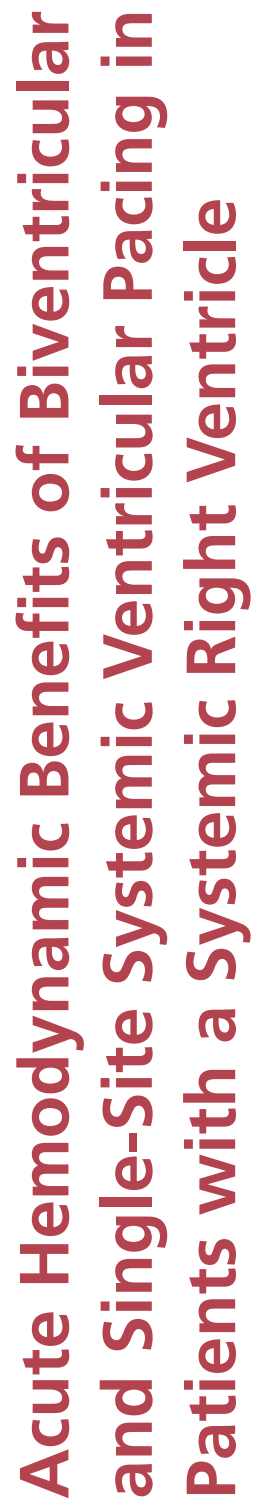




\section{Abstract}

Introduction: Patients treated by atrial redirection surgery (Senning or Mustard procedure) for transposition of the great arteries (TGA), have an important risk for heart failure caused by dysfunction of the systemic RV. Conventional non-systemic ventricular pacing (non-systVP) may even further increase this risk. We investigated whether these patients may benefit from biventricular pacing (BiVP) and/or single-site systemic ventricular pacing (systVP).

Methods: During clinically indicated catheterization in 9 patients with TGA and status post atrial redirection surgery (SenningMustardTGA), endocardial ventricular stimulation (overdrive DDDmode, 80-90 bpm) was applied with temporary pacing leads at the non-systemic and the systemic ventricle. Acute changes in $\mathrm{dP} / \mathrm{dt}_{\max }$ and systolic pressure of the systemic ventricle, as induced by non-systVP, systVP and BiVP compared to reference, were assessed with a pressure wire within the systemic ventricle. Reference was AAI pacing with similar heart rate $(n=7)$, or non-systVP at a lower heart rate than during stimulation at experimental sites (85 vs. 90 bpm; $n=2)$.

Results: systemic $\mathrm{dP} / \mathrm{dt}_{\max }$ and systolic ventricular pressure were significantly higher during systVP $(+15.6 \%$ and $+5.1 \%$, respectively) and BiVP $(+14.3 \%$ and $+4.9 \%$, respectively, compared with non-systVP). In 6 out of 7 patients systemic dP/dt ${ }^{\max }$ was even higher during BiVP and systVP than during AAI pacing.

Conclusions: In a population of patients with SenningMustardTGA, acute hemodynamic effects of endocardial systVP and BiVP were significantly and equally better than those of non-systVP. Single-site systVP and BiVP might also be beneficial in patients with a systemic RV and intrinsic ventricular dyssynchrony. 


\section{INTRODUCTION}

In patients with transposition of the great arteries (TGA) who have undergone atrial redirection by procedures according to Senning or Mustard (SenningMustardTGA) and in patients with congenitally corrected TGA (ccTGA), the systemic circulation is supported by the morphological right ventricle (RV). ${ }^{1}$ Patients with a systemic RV have an important risk for heart failure caused by dysfunction of the systemic ventricle..$^{2-4}$ Conventional left ventricular (LV) pacing (i.e., nonsystemic ventricular pacing; non-systVP) may even further increase the risk for RV failure. Nonsystemic ventricular pacing is associated with decreased exercise capacity and systemic ventricular function and increased inter- and intraventricular dyssynchrony in both patients with normal biventricular anatomy (i.e., concordant connections between the ventricles and the great arteries) and in patients with SenningMustardTGA. ${ }^{5}$ In patients with structurally normal hearts, systemic ventricular function is better in patients with chronic LV pacing (i.e., systemic ventricular pacing; systVP) than in patients with chronic conventional RV pacing. ${ }^{6}$ Furthermore, if such patients present with LV dysfunction, an upgrade of conventional RV pacing to biventricular pacing (BiVP) results in better LV function and a favorable process of reverse remodeling. ${ }^{7,8}$ While in these studies the beneficial effect of BiVP could be due to resynchronization, single site systemic (left) ventricular pacing seems equally beneficial for hemodynamic function as BiVP, despite a lack of reduction in QRS duration..$^{9-11}$ The latter observation suggests that pacing the systemic ventricle creates a better mechanical interaction between the ventricles than pacing the non-systemic ventricle. We investigated the acute effects of endocardial non-systVP, BiVP and single-site systVP on cardiac pump function in patients with SenningMustardTGA.

\section{Methods}

\section{Subjects}

The study was performed according to local ethical guidelines. The study included nine patients with SenningMustardTGA, who underwent a clinically indicated catheterization and gave informed consent for both catheterization and experimental temporary pacing. Patient characteristics are indicated in Table 1.

\section{Pacing protocol}

Temporary pacing leads were introduced via the femoral approach and placed across the baffle in the apex of the non-systemic ventricle and retrograde across the aortic valve against the free wall of the systemic ventricle. During the study protocol, we enforced a constant heart rate by atrial overdrive pacing (80-90bpm) to exclude hemodynamic variability induced by spontaneous changes in heart rate, and to increase the sensitivity of detecting hemodynamic response to changes in pacing-mode. ${ }^{12}$ To allow comparison of pacing at the experimental sites with intrinsic 


\section{Table 1. Patient charateristics}

\begin{tabular}{|c|c|c|c|c|c|c|c|c|c|}
\hline $\begin{array}{l}\text { Patient } \\
\text { number }\end{array}$ & $\begin{array}{l}\text { Type of } \\
\text { surgery }\end{array}$ & $\begin{array}{c}\text { NYHA } \\
\text { functional } \\
\text { class }\end{array}$ & $\begin{array}{c}\text { Age } \\
\text { (years) }\end{array}$ & $\begin{array}{c}\text { PR } \\
\text { duration } \\
\text { (ms) }\end{array}$ & $\begin{array}{c}\text { QRS } \\
\text { morphology }\end{array}$ & $\begin{array}{c}\text { QRS } \\
\text { duration } \\
\text { (ms) }\end{array}$ & $\begin{array}{l}\text { RVEF } \\
(\%)\end{array}$ & $\begin{array}{l}\text { TAPSE } \\
(\mathrm{mm})\end{array}$ & $\begin{array}{l}\text { RV S' } \\
(\mathrm{cm} / \mathrm{s})\end{array}$ \\
\hline 1 (f) & Senning & 1 & 35 & 195 & normal & 120 & 45 & 14 & 10 \\
\hline $2 \quad(m)$ & Mustard & III & 39 & 260 & normal & 120 & 50 & 13 & - \\
\hline $3(f)$ & Senning & III & 34 & 240 & RBBB & 180 & 40 & 13 & 7 \\
\hline $4 \quad(m)$ & Senning & $\|$ & 28 & 160 & RBBB & 140 & 50 & 11 & 9 \\
\hline $5 \quad(m)$ & Mustard & III & 42 & 240 & RBBB & 140 & 25 & 8 & 4 \\
\hline $6 \quad(f)$ & Senning & |II & 17 & 240 (AAI) & RBBB & 145 & 40 & 19 & 9 \\
\hline $7 \quad(\mathrm{~m})$ & Senning & $\|$ & 36 & 150 (DDD) & Paced RBBB & 200 & 40 & 10 & 6 \\
\hline 8 (f) & Senning & $\|$ & 25 & 200 (AAI) & RBBB & 140 & 40 & 15 & 9 \\
\hline $9 \quad(f)$ & Senning & $\|$ & 33 & 200 (DDD) & Paced RBBB & 200 & 40 & 16 & 8 \\
\hline $\begin{array}{c}\text { Total } \\
\text { (median) }\end{array}$ & & $\|-\| \|$ & 34 & 200 & & $140^{*}$ & 40 & 13 & 8 \\
\hline
\end{tabular}

NYHA functional class $=$ New York Heart Association functional class; RBBB $=$ right bundle branch block; RVEF $=$ right ventricular ejection fraction; RV $S^{\prime}=$ systolic tissue velocity of the right ventricular free wall; TAPSE = tricuspid annular plane systolic excursion. * median QRS-duration for patients with intrinsic atrioventricular conduction (patients 7 and 9 excluded)

ventricular activation, atrial pacing only (AAI) was used as reference. In two patients with blocked atrioventricular (AV) conduction (patients 7 and 9), stimulation by the permanent nonsystemic ventricular lead at a lower heart rate than during stimulation at experimental sites (85 vs. 90 bpm) was used as reference. Hemodynamic changes were induced by changing back-andforth from the reference setting to ventricular pacing at the experimental sites; the non-syst ventricle (non-systVP), the systemic ventricle (systVP) or both ventricles (BiVP) (Figure 1). The AVdelay during ventricular pacing had a default value of $120 \mathrm{~ms}$, ensuring complete capture in all patients. Transitions to-and-from experimental ventricular pacing were immediately repeated at least 6 times for each experimental pacing-site.

\section{Data acquisition}

Whilst changing pacing from reference to an experimental pacing-site (and the other way around), we recorded continuous systolic blood pressure and $\mathrm{dP} / \mathrm{dt}_{\max }$ measured invasively from within the systemic (right) ventricle using a 0.014-inch-diameter high fidelity pressure wire (Radi wire, St. Jude Medical, Inc., St. Paul, MN, USA). The pressure wire was introduced retrogradely into the systemic RV via the femoral approach. A continuous ECG signal was recorded using a Dynascope DS-7100 (Fukuda Denshi USA) monitor. Analogue output signals were taken via a 


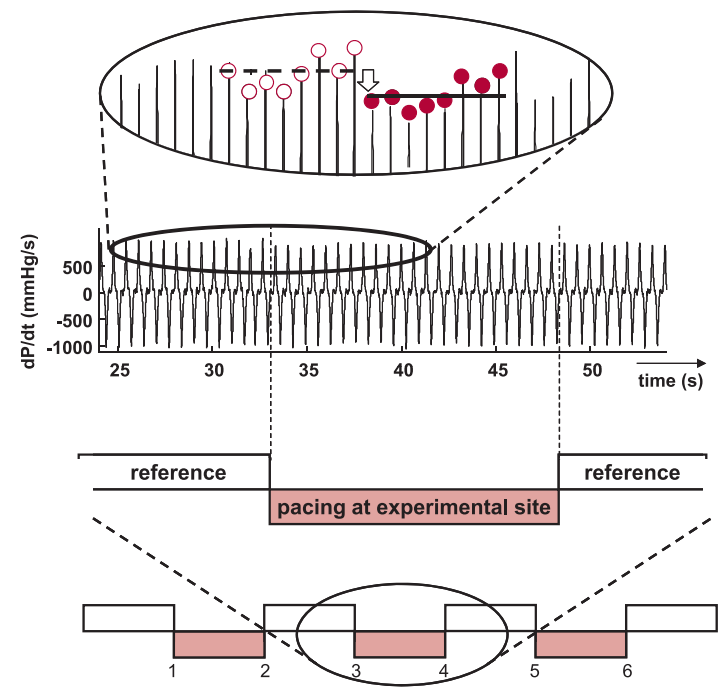

Figure 1. Schematic representation of the protocol

Hemodynamic changes in systolic blood pressure (not in figure) and dP/dtmax (curve) were induced by changing back-and-forth from the reference setting to ventricular pacing at the experimental sites ('transitions'; dashed vertical line). Hemodynamic changes were defined as the difference between the mean of the last 8 beats previous to transition (white dots; dashed horizontal line) and the mean of the 8 beats immediately after transition (black dots; solid horizontal line). For the evaluation of the effect of pacing at the diverse pacing-sites, we used the mean acute change calculated from at least six transitions to and from the pacing-site tested.

National instruments DAQ-Card Al-16E-4 (National Instruments, Austin, TX), acquired in digital form using Labview (National Instruments, Austin, TX), and analysed off line with custom software based on the Matlab platform (MathWorks, Natick, MA). ${ }^{13}$

\section{Outcome parameters and statistical analysis}

Changes in $\mathrm{dP} / \mathrm{dt}_{\max }$ and systolic pressure of the systemic ventricle were defined as the difference in these variables between the mean of the last 8 beats prior to transition and the mean of the 8 beats immediately after transition (Figure 1). For the evaluation of the effect of pacing at the diverse pacing-sites, we used the mean acute change calculated from at least six transitions to and from the pacing-site tested (three forward transitions to pacing at the site tested and three backward transitions to the reference setting, reversing the sign for the back transitions). Paired Student's T-test was used for comparison of the effects of BiVP, systVP and non-systVP. A p-value of $<0.05$ was considered to be significant. 


\section{RESULTS}

\section{Pacing protocol and patients}

Insertion of temporary pacing leads into the systemic ventricle was achieved without complications in all patients. Unfortunately, a computer error in data-recording occurred in one experiment (patient 5). This explains the absence of comparison of systVP versus reference in this patient. Individual protocol conditions and QRS-duration during the different settings are presented in Table 2. Non-systVP increased QRS duration in all patients. BiVP and systVP reduced QRS duration in the patients with prolonged baseline QRS duration (patients 3 and 4) but prolonged it in the other patients, although to a lesser extent than non-systVP.

Table 2. Individual protocol conditions and ECG-findings

\begin{tabular}{cccccc} 
& \multicolumn{5}{c}{ QRS duration (ms) } \\
\hline patient & reference setting & AAl & non-systVP & BiVP & systVP \\
\hline 1 & AAl & 120 & 180 & 140 & 140 \\
2 & AAl & 120 & 240 & 160 & 200 \\
3 & AAl & 170 & 180 & 110 & 120 \\
4 & AAl & 130 & 160 & 120 & 120 \\
5 & AAl & 120 & 160 & 110 & - \\
6 & AAl & 120 & 200 & 160 & 160 \\
7 & Non-systVP 85bpm & - & 180 & 120 & 140 \\
8 & AAl & 100 & 160 & 110 & 110 \\
9 & Non-systVP 85bpm & - & 160 & 120 & 120 \\
\hline AAI = atrial pacing, with inhibition when atrial activation is sensed; BiVP $=$ biventricular pacing; non-systVP = single-site non- \\
systemic ventricular pacing; systVP = systolic ventricular pacing.
\end{tabular}

\section{Acute hemodynamic effects}

The acute changes in systolic ventricular pressure and dP/dtmax of the systemic ventricle, as compared to reference, were significantly better with systVP and BiVP than with non-systVP. Relative changes in dP/dtmax induced by systVP and BiVP were respectively $15.6 \%$ and $14.3 \%$ higher, as compared to those induced by non-systVP. Relative changes in systolic ventricular pressure were $5.1 \%$ and $4.9 \%$ higher for systVP and BiVP, respectively, than for non-systVP. Differences between the effects of BiVP and systVP were not significant. Hemodynamic changes relative to reference and averaged for the study-group, as well as the statistical significance of differences between pacing-sites, are presented in Table 3. The individual and averaged absolute hemodynamic differences of non-systVP, BiVP and systVP versus reference are plotted in Figure 2. 
Table 3. Averaged acute hemodynamic changes relative to reference

\begin{tabular}{|c|c|c|c|c|}
\hline & & non-systVP & BiVP & systVP \\
\hline systemic ventricular systolic & vs. reference & $-2.3 \pm 3.6$ & $2.6 \pm 4.2$ & $2.8 \pm 3.2$ \\
\hline pressure change (\%) & vs. non-systVP * & & $(+4.9 ; p=0.019)$ & $(+5.1 ; p=0.011)$ \\
\hline systemic ventricular & vs. reference & $-6.7 \pm 9.9$ & $7.6 \pm 10.0$ & $9.0 \pm 5.9$ \\
\hline dP/dtmax change (\%) & vs. non-systVP ** & & $(+14.3 ; p=0.001)$ & $(+15.6 ; p=0.001)$ \\
\hline
\end{tabular}
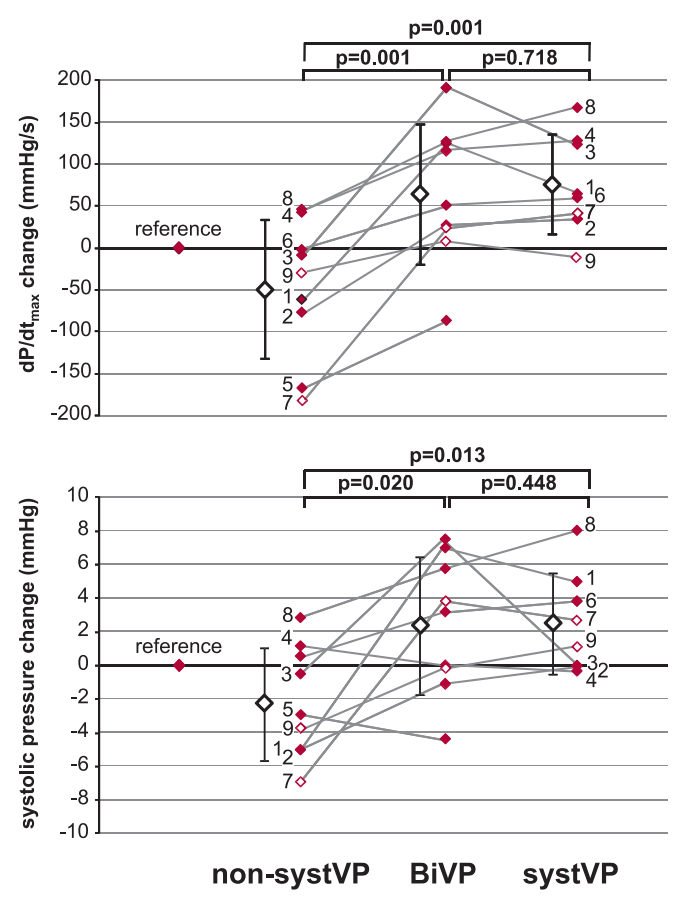

\section{Figure 2. Acute hemodynamic effects of ventricular pacing}

The upper panel shows the acute effects on $\mathrm{dP} / \mathrm{dtmax}$ of the systemic ventricle (i.e., the right ventricle) of pacing at the experimental pacing-sites as compared to reference changes. The lower panel shows the acute effects on systolic pressure of the systemic ventricle. Results for the individual patients are displayed and indicated by patient number. Large diamonds represent mean \pm SD for the total study-group. BiVP = biventricular pacing; non-systVP = non-systolic ventricular pacing (i.e., left ventricular pacing); systVP = systolic ventricular pacing (i.e., right ventricular pacing). 
In 6 out of the 7 patients with intrinsic AV conduction, BiVP and systVP resulted in higher $\mathrm{dP} / \mathrm{dt}_{\max }$ than AAI pacing. Systolic pressure was higher as compared to AAI in four of them with BiVP and in three with systVP.

\section{Discussion}

In the current study in patients with a systemic RV, acute effects of endocardial systVP and BiVP on systolic ventricular pressure and $\mathrm{dP} / \mathrm{dt}_{\max }$ of the systemic ventricle were significantly and equally better than those of non-systVP. Besides, in 6 out of the 7 patients with intrinsic AV conduction, BiVP and systVP resulted in an acute increase in $\mathrm{dP} / \mathrm{dt}_{\max }$ as compared to AAI pacing in reference setting.

\section{Benefit from single-site systemic ventricular pacing and biventricular pacing in comparison to non-systemic ventricular pacing}

Our findings, that both systVP and BiVP are equally superior to non-systVP in terms of acute hemodynamics, suggest that avoidance of delayed activation of the systemic ventricle is important, rather than avoidance of asynchronous electrical activation in general. In both pediatric and adult patients with normal biventricular anatomy (i.e., concordant connections between the ventricles and the great arteries), single-site LV pacing (i.e., systVP) as well as BiVP have been extensively investigated and proven to result in better function of the systemic ventricle, than RV pacing (i.e., non-systVP). 6, 8, 14 Also, in cases with systemic RV, beneficial effects of (upgrade to) BiVP have been observed. ${ }^{15-20}$ However, single-site systVP has never been investigated as alternative pacing therapy in these patients. The current findings indicate that single-site systVP may be at least as good as BiVP for chronic ventricular pacing in patients with systemic RV. Single-site systVP and BiVP may therefore be the preferred sites, not only in patients with a systemic left ventricle, but also in those with a systemic right ventricle. This implies that, in general, pacing should be performed on the ventricle with highest pressure development, irrespective of its morphological type.

\section{Potential benefit from single-site systemic ventricular pacing and biventricular pacing in patients who do not require ventricular pacing for rate control}

Our patients generally had a wide QRS complex. This is in agreement with the prolonged QRSduration (>120 ms) as has been observed in almost half of the patients with SenningMustardTGA or ccTGA. ${ }^{21}$ The wide QRS complex in these patients is often associated with right bundle branch block (RBBB), hence, with delayed activation of the systemic RV. ${ }^{21}$ Chow et al. found a high prevalence of intra-RV (32\%) and interventricular (57\%) dyssynchrony in patients with SenningMustardTGA. ${ }^{22}$ Therefore, in SenningMustardTGA patients without chronic non-systVP, failure of the systemic RV may be (partly) related to intrinsic ventricular 
dyssynchrony. Our observation that BiVP is superior to intrinsic activation in 6 out of the 7 patients with intrinsic AV conduction, is in line with the observations of Dubin et al. and Janousek et al., that patients with either spontaneous or pacing-induced dyssynchrony and RV failure experience positive effects of BiVP. ${ }^{15,23}$ In addition, our experiments indicate that similar results may be achieved by single-site systVP. The potential beneficial effects of BiVP and singlesite systVP in comparison to intrinsic conduction may have been underestimated by our study, because AV delay was not individually optimized. Moreover, results of ventricular pacing versus AAl pacing may have been biased by the influence of differences in AV delay (intrinsic AV delay in AAl versus a default AV delay of 120 ms in ventricular pacing). Therefore, no statistical analysis was performed on the observed differences between intrinsic activation and ventricular pacing at the diverse sites.

\section{Clinical implications and relevance of the study}

The right ventricle is best suited to function as a low-pressure volume pump, rather than having to generate high pressures and to face high resistance, which is inherent to the systemic circulation. Patients with systemic RV are therefore prone to develop heart failure. ${ }^{4,24}$ Identification of novel therapeutic strategies to treat systemic RV dysfunction is of major importance. Moreover, prevention of additional risk factors for heart failure should take first priority in these patients. In this perspective, chronic ventricular pacing should get attention as it may be indicated in about $25 \%$ of the patients with SenningMustardTGA and seems to increase risk for cardiac failure when applied to the non-systemic ventricle. ${ }^{4,5}$ In pediatric patients with AV block and normal biventricular anatomy, cardiac dysfunction related to chronic non-systVP occurs in about $15 \%$ of the patients after mid-term follow-up. 6,25 The ability of the systemic RV to adapt to the stress due to (pacing-induced) dyssynchrony may be limited by its structural abnormality and by the presence of fibrous areas due to surgical scars or chronic ventricular dilatation. ${ }^{26-28}$ Hence, long-term adverse effects of non-systVP and their clinical relevance may be even more important in patients with a systemic RV than in patients with normal cardiac anatomy.

\section{Practical issues of optimal-site pacing in patients with congenital heart disease}

Besides the potential functional benefits, there are several practical issues that have to be taken into account in the decision to apply BiVP or single-site systVP. Implantation approach is an important issue in patients with congenital heart disease. Up to date, (wireless) endocardial pacing within the systemic ventricle is not yet clinically applicable. Stimulation of the systemic ventricle (for systVP or BiVP) should therefore be achieved from the epicardium. Transvenous access to the epicardium of the systemic ventricle via the coronary sinus is difficult to achieve in patients with ccTGA and impossible to achieve in the majority of patients with SenningMustardTGA. However, epicardial stimulation of the systemic ventricle and biventricular stimulation could be achieved with 
a surgical procedure either or not combined with a transvenous approach. Jauvert et al. applied BiVP in seven patients with either cCTGA or SenningMustardTGA and report that BiVP was technically feasible in these patients and that there were no procedure-related complications. ${ }^{29}$ Application of cardiac resynchronization therapy may be added to other necessary cardiac surgical procedures. As suggested by Janousek et al., this may be a good basis for a proactive approach to the prevention of RV failure in this high-risk population. ${ }^{15}$

\section{Limitations of the study}

The present study only assessed acute hemodynamic consequences of pacing at different ventricular endocardial pacing-sites. Studies with permanent pacing and long-term follow-up should be performed to investigate whether BiVP and systVP are clinically to be preferred in all patients with either intrinsic dyssynchrony or an indication for chronic ventricular pacing. It has to be mentioned that the general physical status in the selected study population with clinically indicated catheterization might have been worse than in the overall SenningMustardTGA population..$^{30}$ In the current study we applied endocardial pacing. Recent studies in animals and adult patients suggest that, for comparable sites, endocardial pacing may even be better than epicardial pacing. ${ }^{31,32}$ For chronic ventricular pacing one difficulty of endocardial pacing lies in the access to the cavity of the systemic ventricle. Moreover, the presence of a lead in the systemic ventricle increases the risk of cerebro-vascular accidents by embolism. The application of endocardial pacing in this study may seem therefore a limitation of the study. However, wireless pacing with the use of ultrasound-mediated energy to a receiver electrode is quickly developing and has already been demonstrated in animals and humans in acute studies. ${ }^{33,} 34$

\section{ConcLusion}

In a population of patients with SenningMustardTGA, acute hemodynamic effects of endocardial systVP and BiVP were significantly and equally better than those of non-systVP (i.e., conventional pacing). Single-site systVP and BiVP may therefore be preferred for chronic ventricular pacing in patients with systemic RV, as these may avoid additional risk for RV failure by pacing in these patients. Single-site systVP and BiVP might also be beneficial in patients in whom failure of the systemic RV may be (partly) related to intrinsic ventricular dyssynchrony.

\section{Acknowledgement}

To perform this study, Irene E. van Geldorp received a 'Dr.E.Dekker Grant for Research Fellow in Pediatric Cardiology' from the Dutch Heart Foundation (Grant NHS-2010T078). 


\section{REFERENCES}

1. Senning A. Surgical correction of transposition of the great vessels. Surgery 1959; 45(6):966-980.

2. Graham TP, Jr., Bernard YD, Mellen BG, Celermajer D, Baumgartner H, Cetta F, Connolly HM, Davidson WR, Dellborg M, Foster E, Gersony WM, Gessner IH, Hurwitz RA, Kaemmerer H, Kugler JD, Murphy DJ, Noonan JA, Morris C, Perloff JK, Sanders SP, Sutherland JL. Long-term outcome in congenitally corrected transposition of the great arteries: a multi-institutional study. J Am Coll Cardiol 2000; 36(1):255-261.

3. Dos L, Teruel L, Ferreira IJ, Rodriguez-Larrea J, Miro L, Girona J, Albert DC, Goncalves A, Murtra $\mathrm{M}$, Casaldaliga J. Late outcome of Senning and Mustard procedures for correction of transposition of the great arteries. Heart 2005; 91(5):652-656.

4. Roos-Hesselink JW, Meijboom FJ, Spitaels SE, van Domburg R, van Rijen EH, Utens EM, McGhie $J$, Bos E, Bogers AJ, Simoons ML. Decline in ventricular function and clinical condition after Mustard repair for transposition of the great arteries (a prospective study of 22-29 years). Eur Heart J 2004; 25(14):1264-1270.

5. Horovitz A, De Guillebon M, van Geldorp IE, Bordachar P, Roubertie F, Iriart X, Douard H, Haissaguerre $M$, Thambo JB. Effects of non-systemic ventricular pacing in patients with transposition of the great arteries and atrial redirection. J Cardiovasc Electrophysiol 2012; 23(7):766-770.

6. van Geldorp IE, Delhaas T, Gebauer RA, Frias P, Tomaske M, Friedberg MK, Tisma-Dupanovic S, Elders J, Fruh A, Gabbarini F, Kubus P, Illikova V, Tsao S, Blank AC, Hiippala A, Sluysmans T, Karpawich P, Clur SA, Ganame X, Collins KK, Dann G, Thambo JB, Trigo C, Nagel B, Papagiannis J, Rackowitz A, Marek J, Nurnberg JH, Vanagt WY, Prinzen FW, Janousek J. Impact of the permanent ventricular pacing site on left ventricular function in children: a retrospective multicentre survey. Heart 2011; 97(24):2051-2055.

7. Janousek J, Tomek V, Chaloupecky V, Gebauer RA. Dilated cardiomyopathy associated with dualchamber pacing in infants: improvement through either left ventricular cardiac resynchronization or programming the pacemaker off allowing intrinsic normal conduction. I Cardiovasc Electrophysiol 2004; 15(4):470-474.

8. van Geldorp IE, Vernooy K, Delhaas T, Prins MH, Crijns HJ, Prinzen FW, Dijkman B. Beneficial effects of biventricular pacing in chronically right ventricular paced patients with mild cardiomyopathy. Europace 2010; 12(2):223-229.

9. Blanc JJ, Etienne Y, Gilard M, Mansourati J, Munier S, Boschat J, Benditt DG, Lurie KG. Evaluation of different ventricular pacing sites in patients with severe heart failure: results of an acute hemodynamic study. Circulation 1997; 96(10):3273-3277.

10. Etienne Y, Mansourati J, Gilard M, Valls-Bertault V, Boschat J, Benditt DG, Lurie KG, Blanc JJ. Evaluation of left ventricular based pacing in patients with congestive heart failure and atrial fibrillation. Am J Cardiol 1999; 83(7):1138-1140, A1139. 
11. Auricchio A, Stellbrink C, Block M, Sack S, Vogt J, Bakker P, Klein H, Kramer A, Ding J, Salo R, Tockman B, Pochet T, Spinelli J. Effect of pacing chamber and atrioventricular delay on acute systolic function of paced patients with congestive heart failure. The Pacing Therapies for Congestive Heart Failure Study Group. The Guidant Congestive Heart Failure Research Group. Circulation 1999; 99(23):2993-3001.

12. Whinnett ZI, Davies JE, Willson K, Chow AW, Foale RA, Davies DW, Hughes AD, Francis DP, Mayet J. Determination of optimal atrioventricular delay for cardiac resynchronization therapy using acute non-invasive blood pressure. Europace 2006; 8(5):358-366.

13. Davies LC, Francis D, Jurak P, Kara T, Piepoli M, Coats AJ. Reproducibility of methods for assessing baroreflex sensitivity in normal controls and in patients with chronic heart failure. Clin Sci (Lond) 1999; 97(4):515-522.

14. Vanagt WY, Verbeek XA, Delhaas T, Gewillig M, Mertens L, Wouters P, Meyns B, Daenen WJ, Prinzen FW. Acute hemodynamic benefit of left ventricular apex pacing in children. Ann Thorac Surg 2005; 79(3):932-936.

15. Janousek J, Tomek V, Chaloupecky VA, Reich O, Gebauer RA, Kautzner J, Hucin B. Cardiac resynchronization therapy: a novel adjunct to the treatment and prevention of systemic right ventricular failure. J Am Coll Cardiol 2004; 44(9):1927-1931.

16. Dubin AM, Janousek J, Rhee E, Strieper MJ, Cecchin F, Law IH, Shannon KM, Temple J, Rosenthal E, Zimmerman FJ, Davis A, Karpawich PP, Al Ahmad A, Vetter VL, Kertesz NJ, Shah M, Snyder C, Stephenson E, Emmel M, Sanatani S, Kanter R, Batra A, Collins KK. Resynchronization therapy in pediatric and congenital heart disease patients: an international multicenter study. J Am Coll Cardiol 2005; 46(12):2277-2283.

17. Kirsh JA, Stephenson EA, Redington AN. Images in cardiovascular medicine. Recovery of left ventricular systolic function after biventricular resynchronization pacing in a child with repaired tetralogy of Fallot and severe biventricular dysfunction. Circulation 2006; 113(14):e691-692.

18. Cecchin F, Frangini PA, Brown DW, Fynn-Thompson F, Alexander ME, Triedman JK, Gauvreau K, Walsh EP, Berul CI. Cardiac resynchronization therapy (and multisite pacing) in pediatrics and congenital heart disease: five years experience in a single institution. J Cardiovasc Electrophysiol 2009; 20(1):58-65.

19. Sojak V, Mazic U, Cesen M, Schrader J, Danojevic N. Cardiac resynchronization therapy for the failing Fontan patient. Ann Thorac Surg 2008; 85(6):2136-2138.

20. Janousek J, Gebauer RA, Abdul-Khaliq H, Turner M, Kornyei L, Grollmuss O, Rosenthal E, Villain E, Fruh A, Paul T, Blom NA, Happonen JM, Bauersfeld U, Jacobsen JR, van den Heuvel F, Delhaas T, Papagiannis J, Trigo C. Cardiac resynchronisation therapy in paediatric and congenital heart disease: differential effects in various anatomical and functional substrates. Heart 2009; 95(14):1165-1171. 
21. Diller GP, Okonko D, Uebing A, Ho SY, Gatzoulis MA. Cardiac resynchronization therapy for adult congenital heart disease patients with a systemic right ventricle: analysis of feasibility and review of early experience. Europace 2006; 8(4):267-272.

22. Chow PC, Liang XC, Lam WW, Cheung EW, Wong KT, Cheung YF. Mechanical right ventricular dyssynchrony in patients after atrial switch operation for transposition of the great arteries. Am J Cardiol 2008; 101(6):874-881.

23. Dubin AM, Feinstein JA, Reddy VM, Hanley FL, Van Hare GF, Rosenthal DN. Electrical resynchronization: a novel therapy for the failing right ventricle. Circulation 2003; 107(18):2287-2289.

24. Piran S, Veldtman G, Siu S, Webb GD, Liu PP. Heart failure and ventricular dysfunction in patients with single or systemic right ventricles. Circulation 2002; 105(10):1189-1194.

25. Gebauer RA, Tomek V, Salameh A, Marek J, Chaloupecky V, Gebauer R, Matejka T, Vojtovic P, Janousek J. Predictors of left ventricular remodelling and failure in right ventricular pacing in the young. Eur Heart J 2009; 30(9):1097-1104.

26. Giardini A, Lovato L, Donti A, Formigari R, Oppido G, Gargiulo G, Picchio FM, Fattori R. Relation between right ventricular structural alterations and markers of adverse clinical outcome in adults with systemic right ventricle and either congenital complete (after Senning operation) or congenitally corrected transposition of the great arteries. Am J Cardiol 2006; 98(9):1277-1282.

27. Pettersen E, Helle-Valle T, Edvardsen T, Lindberg H, Smith HJ, Smevik B, Smiseth OA, Andersen K. Contraction pattern of the systemic right ventricle shift from longitudinal to circumferential shortening and absent global ventricular torsion. J Am Coll Cardiol 2007; 49(25):2450-2456.

28. Babu-Narayan SV, Goktekin O, Moon JC, Broberg CS, Pantely GA, Pennell DJ, Gatzoulis MA, Kilner PJ. Late gadolinium enhancement cardiovascular magnetic resonance of the systemic right ventricle in adults with previous atrial redirection surgery for transposition of the great arteries. Circulation 2005; 111(16):2091-2098.

29. Jauvert G, Rousseau-Paziaud J, Villain E, Iserin L, Hidden-Lucet F, Ladouceur M, Sidi D. Effects of cardiac resynchronization therapy on echocardiographic indices, functional capacity, and clinical outcomes of patients with a systemic right ventricle. Europace 2009; 11(2):184-190.

30. Roubertie F, Thambo JB, Bretonneau A, Iriart X, Laborde N, Baudet E, Roques X. Late outcome of 132 Senning procedures after 20 years of follow-up. Ann Thorac Surg 2011; 92(6):2206-2213; discussion 2213-2204.

31. van Deursen C, van Geldorp IE, Rademakers LM, van Hunnik A, Kuiper M, Klersy C, Auricchio A, Prinzen FW. Left ventricular endocardial pacing improves resynchronization therapy in canine left bundle-branch hearts. Circ Arrhythm Electrophysiol 2009; 2(5):580-587.

32. Derval N, Steendijk P, Gula L, Deplagne A, Laborderie J, Sacher F, Knecht S, Wright M, Nault I, Ploux S, Ritter P, Bordachar P, Lafitte S, Reant P, Klein GJ, Narayan SM, Garrigue S, Hocini M, Haissaguerre $M$, Clementy J, Jais P. Optimizing hemodynamics in heart failure patients by systematic screening of left ventricular pacing sites: the lateral left ventricular wall and the coronary sinus are rarely the best sites. J Am Coll Cardiol 2010; 55(6):566-575. 
33. Echt DS, Cowan MW, Riley RE, Brisken AF. Feasibility and safety of a novel technology for pacing without leads. Heart Rhythm 2006; 3(10):1202-1206.

34. Lee KL, Tse HF, Echt DS, Lau CP. Temporary leadless pacing in heart failure patients with ultrasoundmediated stimulation energy and effects on the acoustic window. Heart Rhythm 2009; 6(6):742-748. 


\section{Part l:}

Toward Optimization of Cardiac Resynchronization Therapy

\section{Part II:}

Toward Prevention of Pacing-Induced Heart Failure

Partly adapted from 'Chronic Ventricular Pacing in Children: Toward Prevention of PacingInduced Heart Disease'. I.E. van Geldorp et al.

Heart Fail Rev 2011; 16(3):305-314 


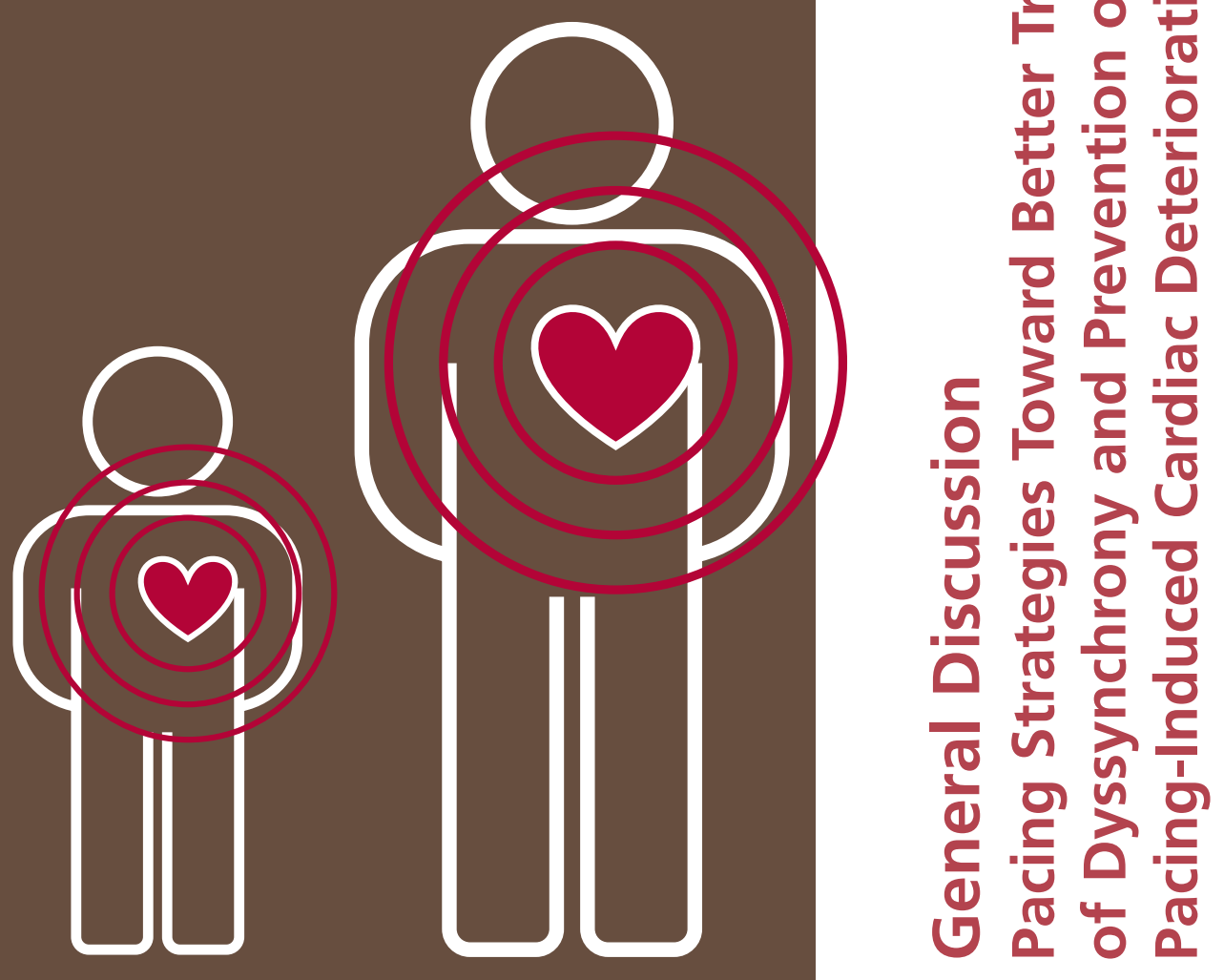




\section{INTRODUCTION}

The general aim of the research presented in this thesis is to better treat electromechanical ventricular dyssynchrony and to avoid pacing-induced cardiac deterioration in adults and children. The motives for the above-mentioned aim are the evident needs for better treatment and prevention of dyssynchrony and cardiac deterioration originating from either intrinsic or pacing-induced left bundle branch block (LBBB), as elucidated in the general introduction of this thesis (chapter 2). These motives are underlined by the studies presented in chapters 3, 5, 6 and 7 showing adverse effects of right ventricular (RV) pacing in patients with normal biventricular anatomy. Clinically more relevant is that the studies presented in this thesis also suggest new pacing-strategies by which cardiac function may be improved and/or preserved.

In this general discussion, the results of the studies presented in this thesis are linked and put in broader perspective. The first part of this chapter discusses optimization of cardiac resynchronization therapy (CRT) in (adult) patients with dyssynchrony-associated cardiac dysfunction. Within this field, patient-selection and the reliability of optimization protocols are discussed in more detail. The second part of the chapter concerns the challenge to prevent pacing-induced dyssynchrony and cardiac deterioration in pediatric cardiology. Several alternative sites for chronic ventricular pacing for rate control are reviewed and clinical implications of the various sites for ventricular pacing in (pediatric) patients with or without structural congenital heart disease are discussed. 


\section{General Discussion part I}

\section{TOWARD OPTIMIZATION OF CARDIAC RESYNCHRONIZATION THERAPY}

Over the last decade, it has been proven that in populations with severe heart failure and electrical dyssynchrony, CRT has the potential to improve left ventricular (LV) function and clinical status, as well as that it can reduce the risk of heart failure-related hospitalization or death. However, individual response to CRT varies widely and as much as 30-50\% of patients selected according to current guidelines respond inadequately. It seems therefore that there is still opportunity for improvement and optimization of this relatively novel therapy.

"CRT optimization" usually refers to tailoring of atrioventricular (AV) and interventricular (i.e, ventriculo-ventricular; VV) delay to the individual patient, aiming at increase of the hemodynamic improvement and long-term benefits induced by CRT. Nonetheless, besides the individual optimization of device-settings, overall response to CRT is also affected by pre-implantation patient selection. All approaches to optimize the response to CRT and the methods to guide these optimizations are currently under debate.

\section{Patient selection affects overall response to CRT}

Typically, CRT is indicated in all patients with wide QRS (QRS $\geq 120 \mathrm{~ms}$ ) and severe heart failure, the latter defined as severely impaired LV function (LV ejection fraction <35\%) and functional status (New York Heart Association functional class III-IV). ${ }^{1}$ Patients are defined as 'responders' if the reduction in LV end-systolic volume after six months CRT is above a certain threshold varying between $10 \%$ or $15 \%$. Unfortunately, it seems that an important proportion of the patients with indication for CRT do not profit from CRT, independent of efforts undertaken to improve individual response. ${ }^{2}$ These latter patients may be concerned as 'real non-responders'. Withholding application of CRT in real non-responders would obviously increase the success rate of CRT and reduce unnecessary implantation costs and complications. Apart from the duration of the QRS complex, its morphology appears also important to identify patients most likely responding to CRT. Patients with classical LBBB are more likely to respond than those with atypical intraventricular conduction delay or right bundle branch block. ${ }^{3}$ Also etiology of heart failure influences response to CRT with better response observed in patients with non-ischemic cardiomyopathy when compared to ischemic cardiomyopathy. ${ }^{4,5}$ Furthermore, many new indices of cardiac dyssynchrony have been proposed to refine pre-implantation patient selection. The clinical value of these indices, however, has not been well established and discussion is going on while new indices are suggested. ${ }^{6,7}$ 
On the one hand CRT might be reserved to a more select subgroup of patients complying with current guidelines, while on the other hand its application might be expanded to other groups of patients. Cardiac resynchronization therapy aims to reverse the deleterious effects that may originate from LV dyssynchrony by restoration of a more coordinated contraction pattern. It seems therefore reasonable to suppose that all patients with LV dyssynchrony associated with LBBB morphology in the ECG would benefit from resynchronization, irrespective of the level of heart failure or nature of LBBB morphology (e.g., intrinsic LBBB, RV pacing). In patients with heart failure following chronic RV pacing, an 'upgrade' from RV pacing to biventricular (BiV) pacing indeed improves clinical presentation, reduces mortality, reverses LV remodeling and improves LV function to the same extent as 'de novo' CRT does in patients with intrinsic LBBB. ${ }^{8-}$ ${ }^{12}$ Distinction between pacing-induced and intrinsic LBBB should therefore no longer be made in the practice of selecting CRT-candidates. ${ }^{1}$ With respect to heart failure classification and response to CRT, major studies (REVERSE and MADIT-CRT) have recently shown that CRT in patients with mild symptoms of heart failure (NYHA I-II) is as effective as in patients with moderate to severe symptoms of heart failure (NYHA III-IV). ${ }^{13,14}$ Although for that reason new American guidelines are less tenacious to the presence of clinical heart failure symptoms, severe LV dysfunction is still a strict criterion: it is advised to consider CRT in slightly symptomatic patients (NYHA II) only if LVEF $<35 \% .{ }^{1}$ Our study presented in chapter 3 of this thesis, revealed that improvement of LV function and reversal of LV remodeling with BiV pacing may occur in patients with even less severe LV dysfunction than reported in the REVERSE study (mean LVEF $36 \pm 10 \%$ versus LVEF $27 \pm 7 \%$, respectively). ${ }^{13}, 15$ Additionally, it seems that the response to BiV pacing is not related to the severity of either $L V$ dysfunction or LV remodeling in the preimplantation period. ${ }^{15}$ These findings indicate that CRT, besides its capability of treatment of dyssynchrony-induced heart failure, may also prevent or slow the progression to severe heart failure in patients with (pacing-induced) dyssynchrony. ${ }^{16}$ However, complication rate and unnecessary costs are important issues in a mildly symptomatic patient cohort and make (upgrade to) BiV pacing in every patient with a conventional pacemaker indication or intrinsic LBBB unfavourable. Until now, neither the long-term effect of (pacing-induced) dyssynchrony, nor the response to CRT can be predicted for the individual patient. Therefore, the application of BiV pacing may better be limited to patients presenting overt echocardiographic signs of remodeling or deterioration of LV function.

\section{Optimization of CRT-response by tailoring of atrioventricular and interventricular delay}

Individual hemodynamic response to CRT and the potential of long-term benefits brought by CRT may be increased by optimization of AV-and $\mathrm{V}$-delay. ${ }^{17-20}$ As optimization of device settings may increase the individual benefit of CRT, it may indirectly also add to the proportion of 'responders'. However, there is lack of consensus on the practice of optimizations of device-settings, as well as 
on the methods and parameters to guide these optimization procedures. Consequently, protocols vary and may not always be well designed. ${ }^{21}$ Interpretation of both optimization studies and clinical optimization procedures is therefore difficult. Moreover, suboptimally designed protocols hamper reliable testing whether or not optimization of device settings is clinically relevant and whether or not the optimum of settings changes over time or during exercise.

There are two general approaches to optimize AV- and VV-delays. One is 'qualitative optimization', which is based on pattern recognition. For example, in Ritter's method and the iterative method for AV optimization, the operator adjusts AV delay until the transmitral Doppler velocity pattern is judged to have the ideal contour. ${ }^{22,23}$ The other approach for optimization of AV- and VV-delays is 'quantitative optimization', in which a physiological variable is measured, either invasively or noninvasively, while timing is adjusted. Doppler measurements are often used in this approach, since they can be made noninvasively. However, even with extensive training, these measurements may be challenging, as they depend on the acoustic window in the patient. Furthermore, it is very time-consuming to obtain a reliable set of data over a series of heartbeats for device optimization. Pressure measurements, such as invasive arterial or ventricular pressure, or its peak slope ( $\left.\mathrm{dP}_{\mathrm{d}} \mathrm{t}_{\max }\right)$ and non-invasive arterial pressure (chapter 4$)$, are less susceptible for patient- or investigator-dependent quality and might more easily be automated.

\section{Reliability of optimization protocols}

Choosing a variable that well reflects hemodynamics and that is easily measured, is not the only thing that matters in the reliability of optimization procedures. Both the amplitude of hemodynamic response and the measurement variability fundamentally determine the precision of an optimization procedure. The amplitude of response depends on the physiological dependence of the variable measured upon changes in device settings, may be very different between AV- and VV- optimization, and may individually be influenced by heart rate. ${ }^{24}$ Moreover, for any given patient, there is variability (i.e., scatter or noise) between replicate measurements at the same setting (Figure 1). This scatter reflects short-term biological variability of the physiological variable, plus measurement error arising from equipment and imperfect observer evaluation. Variability in measurements implicates that simply selecting the AV- or VVdelay at which the highest value is measured (by a single measurement) may not be the optimal approach to maximize the individual benefit of CRT. ${ }^{21}$ Until now no systematic way exists to design optimization protocols to deliver optima to a clinically-desired precision, or even to report confidence intervals. Arbitrary protocol design is risky, because if the random variation between repeat measurements at the same setting (scatter) is large in comparison to the genuine underlying between-setting differences, the protocol may give most patients a wrong recommendation. ${ }^{21}$ This makes execution of the protocol at best a waste of clinical resources and at worst actively harmful. 


\section{FUtURE PERSPECTIVES IN THE OPTIMIZATION OF CRT}

\section{Optimization protocols may be improved}

Improved equipment or improved training may reduce measurement error in optimization protocols. Physiologic variables will, nevertheless, always be affected by intrinsic biological variability. Measurement variability implies that simply selecting the AV-or VV-delay at which the highest value is measured (by a single measurement) is not the optimal approach to maximize the individual benefit of CRT. To decrease the impact of inevitable biological and measurement variability, multiple measurements should be taken and averaged for each device-setting. Since Whinnett et al. demonstrated that the curve of response to BiV pacing at various AV-delays fits closely to a second order polynomial, ${ }^{20}$ it may be a good strategy to fit a parabola to the data points and pick the top of the parabola as the "optimum". By means of statistics, "parabolic-fitting" decreases the total number of measurements needed to reliably define the optimum as compared to the "pickthe-highest" approach. The total number of measurements that should be made to perform a reliable optimization procedure depends on the scatter-to-curvature ratio of the procedure (i.e., ratio between amplitude of response and measurement variability) and the desired precision of the optimization procedure. The highest realistically desirable precision is a $95 \%$ confidence interval of \pm 5 $\mathrm{ms}$. The coarsest precision tabulated is $\pm 30 \mathrm{~ms}$, because poorer precision is very unlikely to be clinically constructive: a $95 \%$ confidence interval of $\pm 40 \mathrm{~ms}$, for example, equates to reporting after optimization that the patient's optimum lies "with 95\% certainty somewhere between 100 and 180 ms". If scatter-to-curvature ratio and desired precision are defined, the number of measurements (combination of settings and repetitions) could be mathematically estimated by the method of Francis et al.(paper in preparation).

Nowadays, echocardiography is still the most commonly used technique to optimize AV- and VVtiming, ${ }^{25}$ yet it is a time-consuming approach and uses significant human resources. The large total number of measurements essential for a reliable optimization procedure, as demonstrated above, may implicate that echocardiography may no longer be feasible for such extensive protocols. In this perspective, non-invasive blood pressure measurements using a finger-cuff, such as Nexfin CO$\operatorname{Trek}^{\circledR}$ (BMEYE B.V., Amsterdam, Netherlands), are very promising. They are inexpensive easy-touse and patient friendly methods that seem highly reliable in the evaluation of hemodynamic changes and would facilitate the assessment of as many measurements as desired (chapter 4). ${ }^{26}$ For the sake of application in routine clinics it would however be desirable to have an algorithm that allows automated execution of the optimization procedure.

\section{Improvement in the delivery of CRT}

With reliable and easy optimization protocols, it would be possible to optimize CRT delivery for all patients. Initially, AV- and VV-delay optimization procedures could be included as part of 

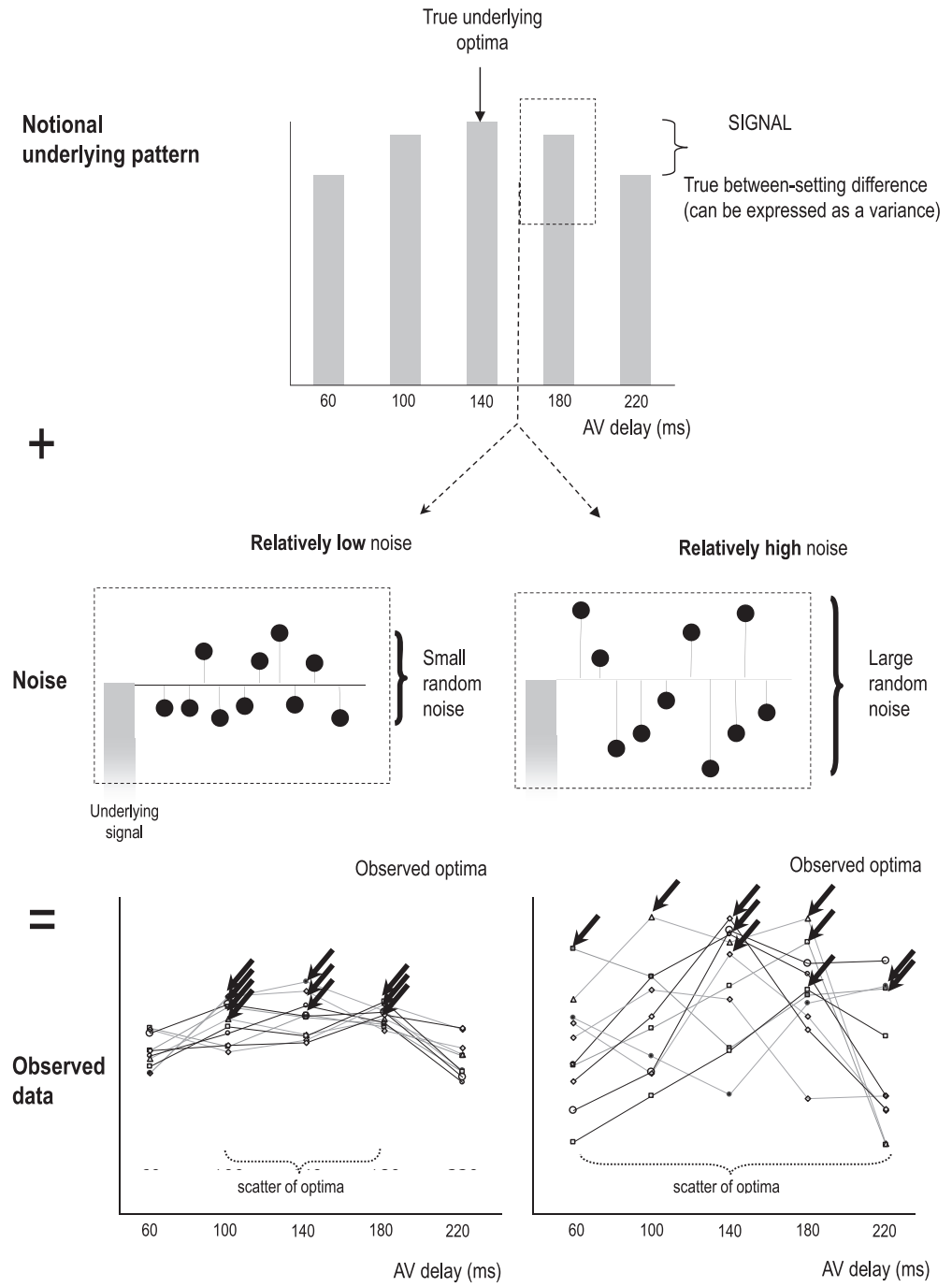

\section{Figure 1.}

Observed measurements are composed of underlying true difference between settings ("signal", top panel) and beat-to-beat variability ("noise", middle panel), which may be small (left) or large (right) relative to the signal. The relative sizes of underlying signal and overlying noise determine whether the observed measurements (bottom panel) reflect the underlying signal faithfully (left) or not (right). When the noise variability is relatively large (right), the observed optimum in a single measurement series (arrow) is often not the true optimum (140 ms in all cases in this figure). (After Pabari et al. Heart Fail Rev. 2011., with permission) $)^{21}$ 
routine short-time follow-up after device implantation. Moreover, it would be interesting to optimize settings at various heart rates and during exercise. ${ }^{27,}{ }^{28}$ Furthermore, delivery of CRT may also be optimized by testing various lead locations for their hemodynamic effects during CRT implantation. The latter approach might be especially beneficial in patients with abnormal cardiac anatomy or patients with scar tissue due to cardiac ischemia. ${ }^{29-32}$ One of the major advantages of using non-invasive blood pressure measurements is that it can easily be applied in different body positions and even during exercise. Prospective randomized studies are needed to clarify the real clinical impact of optimization of CRT-delivery on the patients course. In this perspective, precision of the protocol is an essential prerequisite for (1) interpreting apparent optima, (2) interpreting changes over time or apparent differences between alternative optimization methods, and (3) planning long-term outcome studies.

\section{CONCLUSIONS AND SUgGestions fOR OPTIMIZATION OF CRT}

Improvement of clinical condition and improvement in echocardiographically derived variables in patients with mild heart failure (chapter 3$)^{15}$ suggest that progression to severe heart failure in patients with electromechanical dyssynchrony may be prevented or slowed down by CRT. ${ }^{16}$ Until now, neither the long-term effect of (pacing-induced) dyssynchrony, nor the response to CRT can be predicted for the individual patient. Complication rate and unnecessary costs are important issues in a mildly symptomatic patient cohort and make (upgrade to) BiV pacing in every patient with a conventional pacemaker indication or intrinsic LBBB debatable. Therefore, careful monitoring of $L V$ function is advisable and (upgrade to) BiV pacing should be considered when echocardiographic signs of remodeling or deterioration of LV function occur.

Individual optimization of the device settings might increase the beneficial response to CRT. Though, the method for optimization seems to be of critical importance for the reliability of its outcome. Protocol design with adequate precision is essential to prevent optimization from wasting clinical resources and harming patients by applying a setting that is far from optimal. Non-invasive blood pressure measurements using a finger-cuff, such as Nexfin CO-Trek ${ }^{\circledR}$ (chapter 4), ${ }^{26}$ may be very useful in the optimization of CRT, in both clinics and research. 


\section{General Discussion part II}

\section{TOWARD PREVENTION OF PACING-INDUCED HEART FAILURE}

While the majority of patients receiving pacemakers are adults at usually advanced age, a small group of pacemaker recipients are children, even newborns. Results and conclusions from pacing studies in adults are not readily transferable to the pediatric population, because diseases and causes of dyssynchrony and heart failure differ strongly. In adults, indication for pacemaker therapy or CRT concerns, among others, bradycardia, LV dysfunction, and dyssynchrony due to partially diseased conduction systems often combined with degenerative diseases and infarctions of the myocardium. In children and young adults, indication for pacemaker therapy typically concerns bradycardia due to complete atrioventricular conduction block. This condition may exist either "isolated" in structurally normal hearts or combined with abnormalities in cardiac anatomy. In patients that have a perspective of life-long ventricular pacing for AV block, the prevention of functional as well as structural deterioration seems a major challenge in addition to the restoration of heart rate.

Recognition of potentially harmful effects of chronic RV pacing (chapter 2) ) $^{33-35}$ has prompted investigation into alternative ventricular pacing-sites. In addition to the traditional RV apex and free wall sites, several other sites within the RV became accessible by advances in pacemakerlead technology and implant approaches and began to be clinically explored. Also, biventricular pacing has been introduced in children. Surgical approaches are often preferred for all lead implantations in small children or children undergoing cardiac surgery, implicating a great extent of freedom to position the lead(s) at the epicardium of either the RV or the LV, or even at both.

\section{Alternative-site RV pacing}

Both terms 'alternative-site $R V$ pacing' and 'selective-site $R V$ pacing' refer to pacing at sites other than the RV apex or free wall, though either located within or approached via the RV. These sites are selected based on the (individual) prospect of a more physiological electrical activation pattern and a better hemodynamic response with less detrimental remodeling. By lead insertion through the RV, the His bundle may be paced directly. In patients without distal conduction abnormalities, His-bundle pacing would obviously induce a normal physiological sequence of activation and therefore prevent the heart from dyssynchronous activation and associated harmful effects. Indeed, beneficial effects of successful His-bundle pacing have been reported in adults with AV-nodal ablation for atrial fibrillation. ${ }^{36,37}$ Although technical advances have improved the success rate of His-bundle pacing, ${ }^{38}$ implantation in this very small region is a challenging procedure and seems not very applicable in children. Moreover, especially in children with surgically induced AV block, but also in children with congenital AV block, the His bundle 
may be involved in the pathological interruption of the conduction system, implicating that there is no rationale left for direct His-bundle pacing. Within the field of research on alternative RV pacing sites, also the RV outflow tract has been extensively investigated. Unfortunately, the term RV outflow tract pacing is not always clearly defined in literature and has been used to describe a variety of RV pacing sites. Nonetheless, differentiation between the diverse sites within the conical RV outflow tract is very important, as activation patterns and wave propagation will differ depending on the exact anatomical position of the lead. ${ }^{39}, 40$ Not surprisingly, studies with regard to the effects of RV outflow tract pacing without specification of exact anatomical definition of the site have shown inconsistent results. ${ }^{41,42}$ Beneficial effects for RV outflow tract pacing have most consistently been found in studies clearly mentioning to pace from the septal side of the RV outflow tract (also defined as "high septal" or "para-Hisian" pacing). ${ }^{43-49}$ Thus, in RV outflow tract pacing, in particular the septal side of the outflow tract may be a target. Nevertheless, it should be noted that 'septal RV outflow tract' is not a good definition, since the upper part of the 'septal side' within the RV outflow tract is located above the LV. Therefore, only the inferior part of the septal side of the RV outflow tract can be considered as truly septal and might be the preferred location for lead placement in the RV outflow tract. ${ }^{39,}, 40$

\section{Biventricular pacing}

Data from large randomized adult CRT trials cannot simply be translated to children with cardiac failure, because the pediatric population not only includes children with normal cardiac anatomy and LV failure but also includes children with univentricular hearts (with either RV or LV morphology), hearts with a systemic RV (i.e., heart with either congenitally corrected transposition of the great arteries or transposition of the great arteries with atrial redirection surgery in the past), and hearts with RV failure (e.g., RV failure associated with corrected Tetralogy of Fallot). In addition, a substantial number of pediatric CRT candidates have ventricular dyssynchrony and cardiac failure related to conventional pacemaker therapy for postoperative or congenital AV block. Nonetheless, encouraged by the positive results in adults, BiV pacing meanwhile has also been applied in children with ventricular dysfunction. In these children with either isolated AV block or AV block combined with structural heart disease, BiV pacing (often following chronic RV pacing) improved pump function and reversed ventricular remodeling. Upgrade from single-site RV pacing to BiV pacing was in children at least as effective as in adults with heart failure. In some children, even the need for cardiac transplantation was deferred. ${ }^{50,51}$ From the analysis of Janousek et al., the response to BiV pacing seems to depend on the structural and pathophysiological substrate. The response was most favorable after upgrades from single-site RV pacing to BiV pacing in patients in whom the LV was the systemic ventricle. ${ }^{51}$ Noteworthy, in these studies, resynchronization was mostly indicated for decreased LV function following chronic conventional RV pacing. ${ }^{51,52}$ 


\section{Single-site left ventricular pacing versus biventricular pacing}

In children with LV dysfunction following chronic RV pacing, functional improvement and reverse remodeling have also been reported for LV single-site pacing. ${ }^{53,}{ }^{54}$ Single-site LV pacing in adult heart failure patients resulted in the same improvement in LV function as induced by acute or chronic BiV pacing. ${ }^{55-57}$ It is argued that single-site LV pacing in LBBB patients results in "hidden resynchronization" by fusion of the activation front originating from the left lateral pacing electrode with the impulse traveling through the right bundle. However, because benefits of single-site LV pacing are also observed during pacing with short AV delays ${ }^{57}$ as well as in patients with atrial fibrillation, ${ }^{56}$ it is very unlikely that the hypothesis of "hidden resynchronization" applies to all patients. In dogs with experimental complete AV block, Mills and colleagues showed that LV apical and LV septal pacing resulted in only moderate electric desynchronization as well as in minor redistribution of mechanical work and perfusion. ${ }^{58}$ Even after 4 months of pacing in these dogs, LV pacing induced normal levels of contractility, relaxation, and myocardial efficiency. Moreover, single-site LV apical and LV septal pacing maintained cardiac function and efficiency at least as well as BiV pacing. ${ }^{58}$ Previous to the above-mentioned study, it had already been shown in laboratory dogs and in children undergoing cardiac surgery that LV pacing acutely increases pump function when compared with $\mathrm{RV}$ pacing. ${ }^{59}$

\section{Single-site left ventricular pacing may prevent pacing-induced heart failure}

Based on surgical preferences, chronic single-site LV pacing has in some centers clinically been applied in children for already several years. Results of small studies in children of one of these centers suggested that chronic LV free wall pacing may preserve LV function and dimension at a normal level (chapter 5). ${ }^{54,60,61}$ Encouraged by these results, we retrospectively and crosssectionally evaluated long-term influences of single-site RV and LV pacing sites on LV function and dyssynchrony in structurally normal hearts in a multi-center study (chapters 6 and 7). ${ }^{62,63}$ Results from these evaluations indicate that the site of pacing is indeed an important determinant of LV function, with LV pacing consistently resulting in better LV function than RV pacing and with depressed LV function seen in over $10 \%$ of the RV-paced patients. In the individual patient, depressed LV function may indicate that chronic pacing is not well tolerated and it may impose a higher risk for pacing-induced heart failure. Both LV function and contraction efficiency were significantly better in children with chronic LV pacing than in children with chronic RV pacing. ${ }^{62,}{ }^{64}$ Interventricular and intra-LV dyssynchrony were minimal in children undergoing LV apical or LV lateral wall pacing. As suggested by the cross-sectional data (chapter 7), as well as by the retrospective data presented in Figure 2 (unpublished data), LV function is best preserved in children undergoing $L V$ apical or lateral wall pacing. However, reliable statistical comparison between all individual pacing-sites is disputable in this study-population, because data is limited for several pacing-site subgroups. 


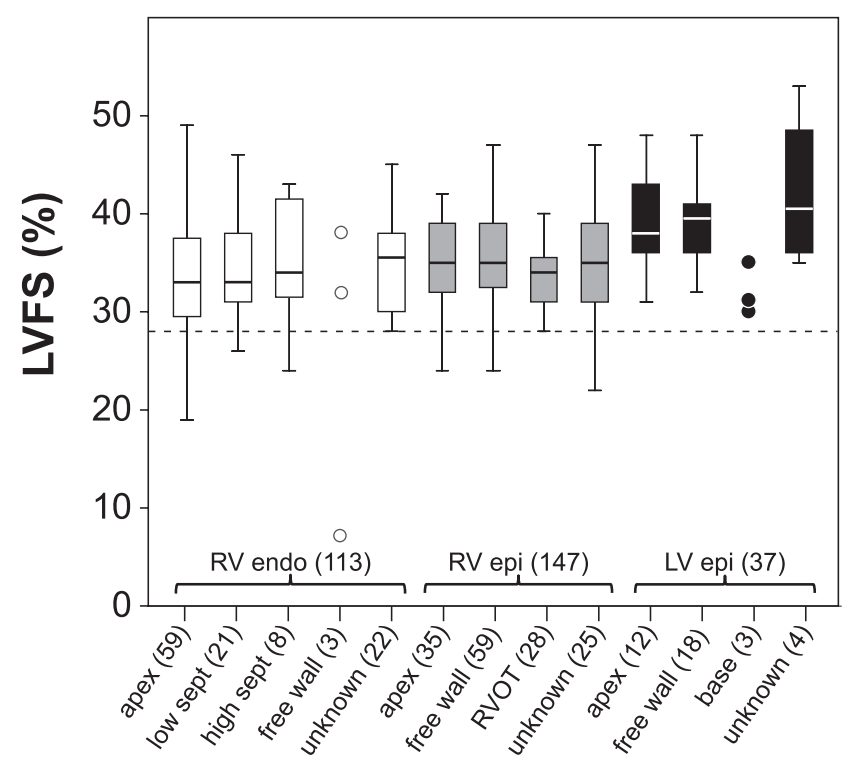

pacing site

Figure 2. Left ventricular fractional shortening for pacing-site subgroups

Left ventricular fractional shortening (LVFS) is displayed for every pacing-site subgroup (the numbers of patients for each location are indicated between brackets). No statistical analyses were performed on the differences between these subgroups. Boxplots represent $25^{\text {th }}, 50^{\text {th }}$, and $75^{\text {th }}$ percentiles. Whiskers represent the minimal and maximal values within the range of $\left[25^{\text {th }}\right.$ percentile $-1.5^{*}$ InterQuartileRange] and $\left[75^{\text {th }}\right.$ percentile $+1.5^{*}$ InterQuartileRange], respectively. Dots represent individual values.

Apex $=$ ventricular apex; Base $=$ base of the ventricle; RVOT $=$ right ventricular outflow tract; Sept $=$ interventricular septum. Unknown = the precise location of the electrode, within/at the paced ventricle, was unknown. (Unpublished data from van Geldorp et al.; chapter 6)

\section{The effect of pacing-site and the influence of maternal autoantibodies}

Congenital AV block is strongly associated with the presence of maternal $\lg G$ autoantibodies (anti-SSB/La and anti-SSA/Ro). These autoantibodies possibly react with SSB/La and SSA/Ro antigens, thereby triggering an inflammatory response in the fetus that typically leads to fibrosis and scarring of the conduction system. ${ }^{65}$ The inflammatory response may however also lead to a more diffuse myocardial disease. ${ }^{66,67}$ Since most research on the effect of pacing-site has been done in populations including children with congenital AV block, the question has been raised whether the outcomes for the effects of pacing-site may have been biased by maternal autoantibody status. 
In the retrospective part of our multi-center study we did not find any significant influence of maternal autoantibody status on LV function, while some sub-analyses of the cross-sectional study suggest an influence of maternal autoantibody status. When considering both the retrospective and cross-sectional evaluations, it seems that the influence of maternal autoantibody status hangs in the balance of significance. Theoretically, this might implicate that there is indeed an association between LV function and maternal autoantibody status, but the study population is not large enough to strongly indicate this association. Clear is, however, that the influence of maternal autoantibody status, if there is any, is less important than the influence of the site of pacing. Pacing the ventricle at the best spot is highly important in the entire population, but is possibly even more important for those patients having positive maternal autoantibody status.

\section{Optimal pacing in congenital heart disease}

The ever-improving survival after surgery for complex congenital heart disease broadens the variety of anatomical substrates and indications for chronic ventricular pacing. The pediatric pacing-population is therefore highly hetero-geneous in terms of age and cardiac anatomy. Up to date, most comparisons of various pacing-sites in children have been made in a population with structurally normal hearts. Depending on the morphological, electrical and functional anomalies associated with each congenital heart disease, it is probable that in patients with congenital heart disease, pacing at a certain site has dissimilar effects than pacing at a comparable site in patients with isolated AV block. ${ }^{68-71}$ We assume that the optimal pacing-sites depend on cardiac substrates, which include cardiac anatomy and the presence of intrinsic dyssynchrony. For example, in patients with RV failure and intrinsic dyssynchrony from a right bundle branch block after surgically repaired Tetralogy of Fallot, RV pacing has been proposed as a potential therapy for RV failure. ${ }^{72-74}$ It has however been suggested that, when both RV and LV function are concerned, BiV pacing may be superior to RV pacing. ${ }^{75,76}$ Further investigations after the effects of long-term ventricular pacing are required to confirm these findings and to determine whether pacing the late-activated ventricle (i.e., RV) or BiV pacing in these patients is worthwhile. ${ }^{77,78}$

In patients with congenitally corrected transposition of the great arteries or transposition and status post atrial redirection surgery, the morphological RV supplies blood-flow to the body, instead of the morphological LV. In this group of patients LV pacing (i.e., non-systemic ventricular pacing) is associated with lower functional and exercise capacity, lower systemic ventricular function and higher degree of inter- and intraventricular dyssynchrony. ${ }^{79}$ Adverse effects induced by pacing the non-systemic ventricle may be of major clinical importance since they may induce additional risk for dysfunction of the systemic RV in these patients who do already have high risk for cardiac dysfunction. We found in patients with status post Senning or Mustard procedure for transposition of the great arteries that acute hemodynamic effects of endocardial systemic ventricular pacing and biventricular pacing were significantly and equally better than those of non-systemic ventricular pacing (chapter 8). Single-site systemic ventricular pacing and 
biventricular pacing may therefore be preferred for chronic application of ventricular pacing, not only in patients with a systemic left ventricle, but also in those with a systemic right ventricle.

\section{Why systemic ventricular pacing is superior over non-systemic ventricular pacing}

We postulate that, above and beyond synchrony, the sequence of electrical activation is a major determinant of cardiac pump function. ${ }^{80,81}$ During LV free wall pacing in patients with isolated AV block, the prolonged duration of total activation is comparable with the delay during RV pacing, as reflected by a similar prolongation of the QRS duration. ${ }^{60}$ However, during LV pacing, the LV lateral wall is activated prior to the septum and RV lateral wall, preventing the septum from paradoxical movements and resulting in superior hemodynamic performance as compared to RV pacing. ${ }^{82}$ Left ventricular apical pacing induces a physiological apex-to-base sequence of activation that results in synchronous electrical activation and contraction at the circumferential level of the LV. ${ }^{83,84}$ Compared with other RV and LV sites, LV apical pacing preserves septal to lateral LV synchrony and systolic function. ${ }^{63,64,85}$ Our findings in patients with a systemic RV, that both systemic ventricular pacing and BiV pacing are equally superior to non-systemic ventricular pacing in terms of acute hemodynamics, again indicate that the sequence of activation is important, rather than avoidance of asynchronous electrical activation in general. With regard to the activation sequence, all data point towards the idea that the electrical impulse should start at the chamber with the higher developed pressure, irrespective of its morphology. This also indicates that mechanical interaction between the chambers is a major determinant of the hemodynamic effect of ventricular pacing. Furthermore, optimal single-site pacing may be at least as good as BiV pacing.

\section{Practical considerations with respect to optimal pacing}

In patients with (complex) congenital heart defects, restrictions in lead placement could be introduced by anatomy, prior surgery or scarred myocardium. In older children and adult patients, leads are often transvenously inserted and endocardially positioned at the apex or septum of the non-systemic ventricle, because it is technically safe, fast, and reliable. In neonates and small children, most centers preferably use epicardial lead placement by a surgical approach. Epicardial lead placement is also preferred in children with an open connection to the systemic ventricle, because in this condition the presence of endocardial leads increases the risk of cerebrovascular accidents by embolism. Since possibly successful alternative RV pacing sites require a transvenous approach for lead implantation, these sites are probably not feasible in small children. For the purpose of single-site LV pacing, the epicardial LV apex or, alternatively, the LV free wall can easily be approached through a (limited) sternotomy, sub-xiphoidal incision or left lateral thoracotomy, ${ }^{86}$ with good lead stability and pacing performance, as well as excellent cosmetic results. In larger children and adults, in whom a transvenous approach may be preferred, single-site LV and BiV pacing may also be achieved by implantation via the coronary sinus, though for single-site LV pacing it is recommended to position the ventricular lead as far 
to the apex as possible (chapter 5). ${ }^{59,60}$ A major practical advantage of single-site pacing, compared with BiV pacing, is that only one ventricular lead is needed. This will prolong battery longevity and reduce potential vascular problems and lead-associated complications. Because every (re)placement of either a lead or generator comes with risks of complications, the number of replacements should be minimized, especially in children, since they have a perspective of lifelong pacing. Thus, single-lead pacing should be seriously considered.

Given the absence of a consistent correlation between cardiac function and paced QRS duration in acute and chronic pacing studies, we discourage the use of QRS duration as a tool for the selection of an optimal epicardial pacing site in children. ${ }^{59,60,87,88}$ A better approach in this respect may be the use of the pattern of activation assessed by non-invasive epicardial electrocardiographic imaging, ${ }^{89,90}$ although the optimal activation pattern for the diverse anatomical substrates is not known yet. Therefore, and since the intention of optimal-site pacing is to preserve cardiac function, it may be even better to assess pump function by either (noninvasive) hemodynamic or echocardiographic measurements while pacing from various pacing sites, and implant the lead at the site where pacing results in the best pump function.

\section{Future PERSPECtives in the PREVENTION OF PACING-INDUCED CARDiaC DETERIORATION}

\section{Increase of the level of evidence}

Our studies investigating the effect of pacing-site on LV systolic function (chapters 5-7) have shown that LV pacing is superior to RV pacing in patients with isolated AV block, and that none of the LVpaced patients was in heart failure. ${ }^{60,62,64}$ Although pacing-duration in our multi-center study was reasonably long (about 5 years), future studies with very long-term longitudinal follow-up and larger numbers of LV-paced patients are needed to demonstrate that LV pacing indeed preserves LV systolic function and prevents pacing-induced heart failure. Additionally, the effect of pacing-site on parameters other than LV function should be explored. Furthermore, since functional improvement and reverse remodeling in patients with LV dysfunction following chronic RV pacing have been reported for both LV single-site pacing ${ }^{53,54}$ and BiV pacing, ${ }^{50,51}$ it should be investigated whether or not a clinically relevant difference exists in effect of these pacing-therapies in the treatment of pacing-induced heart failure. Randomized controlled trials are the most rigorous way of determining whether a cause-effect relation exists between treatment and outcome. However, practical concerns such as the relatively low incidence of isolated complete AV block and high number of patients needed for statistical power, limits controlled randomization. Therefore, controlled randomization seems not the most feasible design for future studies to compare long-term effects of various pacing therapies in children. Moreover, at the current level of evidence, it may be debated whether it would be ethical to randomly apply epicardial RV and LV pacing. 


\section{Differentiation between left ventricular pacing sites: apex versus base.}

It is of clinical importance, once LV epicardial pacing-sites will be more often applied, to know which site, apex or free wall, should be preferred. Left ventricular apical pacing induces a physiological apex-to-base sequence of activation, which results in synchronous electrical activation and contraction at the circumferential level of the LV and seems to preserve septal to lateral LV synchrony and systolic function. ${ }^{83-85}$ In the small dog experiment with paired comparison of LV pacing-sites, described in the 'discussion' of chapter 5, the hemodynamic benefit of LV pacing gradually decreased as compared to LV apical pacing when pacing was applied at sites closer to the base of the LV free wall. ${ }^{59,60} \mathrm{~A}$ similar trend was observed in the population of the multi-center survey described in chapters 6 and 7 (Figure 2). These data suggest that the apex of the LV is indeed the optimal site for ventricular pacing. However, the optimal site is not always the "preferred site" for chronic ventricular pacing when practical aspects, such as established minimal surgical techniques, are concerned. Pacing at epicardial LV mid lateral wall sites, easily accessible through a left lateral thoracotomy, also resulted in good LV function and may clinically be not inferior to LV apical pacing. It should be further investigated whether or not there are clinically relevant differences between the various pacing-sites at the LV. To facilitate the application of "optimal site pacing" in clinical practice, it would be helpful when a range of functionally "preferred pacing sites" could be defined.

\section{Endocardial and leadless pacing}

Recent studies in animals and adult patients suggest that, for comparable LV sites, endocardial pacing may even be better than epicardial pacing. ${ }^{29,}$ 91, 92 One difficulty of endocardial pacing lies in access to the cavity of the systemic ventricle. So far, in patients with normal biventricular anatomy, most endocardial leads have been placed with a transseptal approach that accesses the LV cavity passing from the right atrium through the left atrium. Secondly, the presence of a lead in the systemic ventricle increases the risk of cerebro-vascular accidents by embolism. Recently, leadless pacing with the use of ultrasound-mediated energy to a receiver electrode has been proposed and has already been demonstrated in animals and humans in acute studies. ${ }^{93,94}$ Another proposed approach is to use magnetic induction. ${ }^{95}$ By the use of a "wireless" electrode, implanted via a trans-aortic access, pacing from any of the diverse endocardial sites in the systemic ventricle could be achieved. Besides the possible functional advantages of leadless pacing, elimination of the lead would also exclude lead complications, which nowadays are still common, especially in growing children. 


\section{CONCLUSIONS AND SUGgESTIONS FOR OPTIMAL PACING}

\section{"primum non nocere"; first do not harm}

Children are generally paced from a very early age and typically have a perspective of life-long pacing. Recognizing the adverse effects of dyssynchrony, treatment, or, preferably, prevention of pacing-induced dyssynchrony, must take high priority in these patients. Concerning preservation and restoration of pump function, both BiV pacing and single-site systemic ventricular pacing seem promising in the young. Here raises the question whether or not such pacing therapies should be applied in all children with an indication for chronic ventricular pacing. When choosing the optimal pacemaker therapy and pacing-site for the individual patient, one should discriminate between pacing approaches aiming at the prevention of dyssynchrony and the ones that resynchronize (treat dyssynchrony). Additionally, practical and functional rationales for ventricular lead positions should be taken into account.

\section{Optimal pacing in patients with or without structural congenital heart disease}

Based on the (patho)physiological background of ventricular pacing, on the current knowledge regarding the various pacing-sites, as well as on clinical and practical aspects, some recommendations and suggestions are given for pacing in both patients with normal cardiac anatomy and patients with structural heart defects. These suggestions are also schematically displayed in Figure $3 A$ and $B$, respectively. ${ }^{96}$

For chronic ventricular pacing in patients with normal biventricular anatomy these suggestions are the following (Figure 3A); first of all, because by definition, ventricular pacing alters the physiological pattern of electrical activation, ventricular pacing should be avoided (or minimized) in patients with (partially) preserved AV conduction and intact His-Purkinje system, such as Sick Sinus Syndrome. ${ }^{97,} 98$ In patients with chronic ventricular pacing for surgically induced AV block, the existence of underlying ventricular rhythm by re-established AV conduction should be regularly checked, as very late recovery of $\mathrm{AV}$ conduction is known to occur. ${ }^{99}$ In children with structurally normal hearts without intrinsic dyssynchrony, we advocate the use of single LV apex and LV free wall sites as the preferred sites for ('de novo') chronic ventricular pacing for AV block (chapters 5-7), especially if a surgical approach is also practically advised. ${ }^{60-62,64,100} \mathrm{It}$ is recommended to preferably avoid pacing from the RV free wall, both endocardially and epicardially. ${ }^{63,} 101$ However, in young adults receiving a first pacing system or having a system replacement, the routine transvenous approach seems justifiable in the context of practical aspects, because RV apical pacing is well tolerated by most patients. ${ }^{102,103}$ 


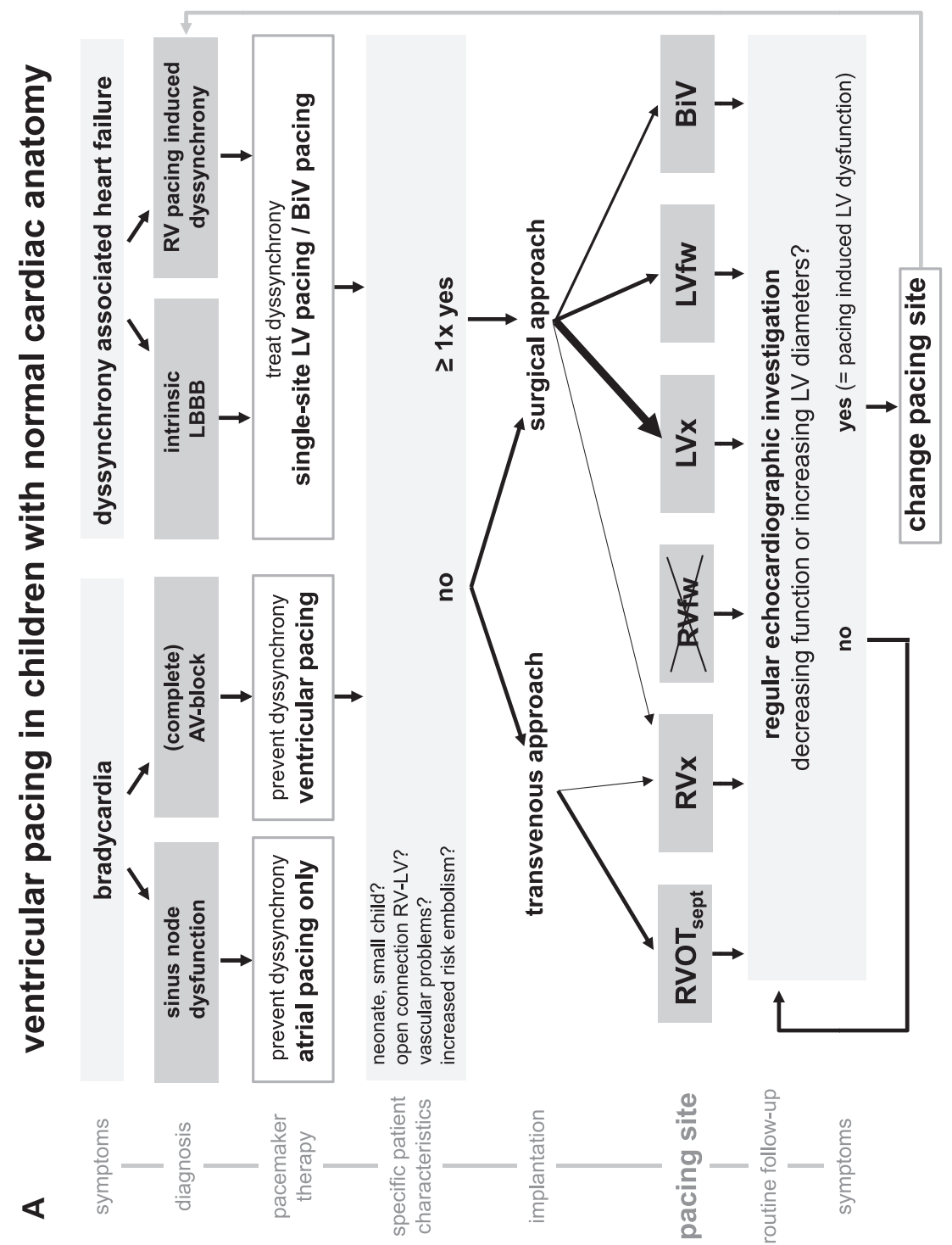

Figure 3. Recommendations for optimal pacing; a schematic overview

$\mathrm{AV}$ block = atrioventricular block; BiV = biventricular $=\mathrm{RVx}($ or RVfW $)+\mathrm{LVfW} ; \mathrm{LBBB}=$ left bundle branch block; $L V=$ left ventricle/ventricular; $L \mathrm{LVW}=\mathrm{LV}$ free wall; $\mathrm{LVx}=\mathrm{LV}$ apex; $\mathrm{RBBB}=$ right bundle branch block; $\mathrm{RV}=$ right ventricle/ventricular; $\mathrm{RVfw}=\mathrm{RV}$ free wall; $\mathrm{RVOT}_{\text {sept }}=$ the inferior part of the septal side of the RV outflow tract; RVx $=$ RV apex. (From van Geldorp et al. Heart Fail Rev 2011., with permission) ${ }^{96}$ 


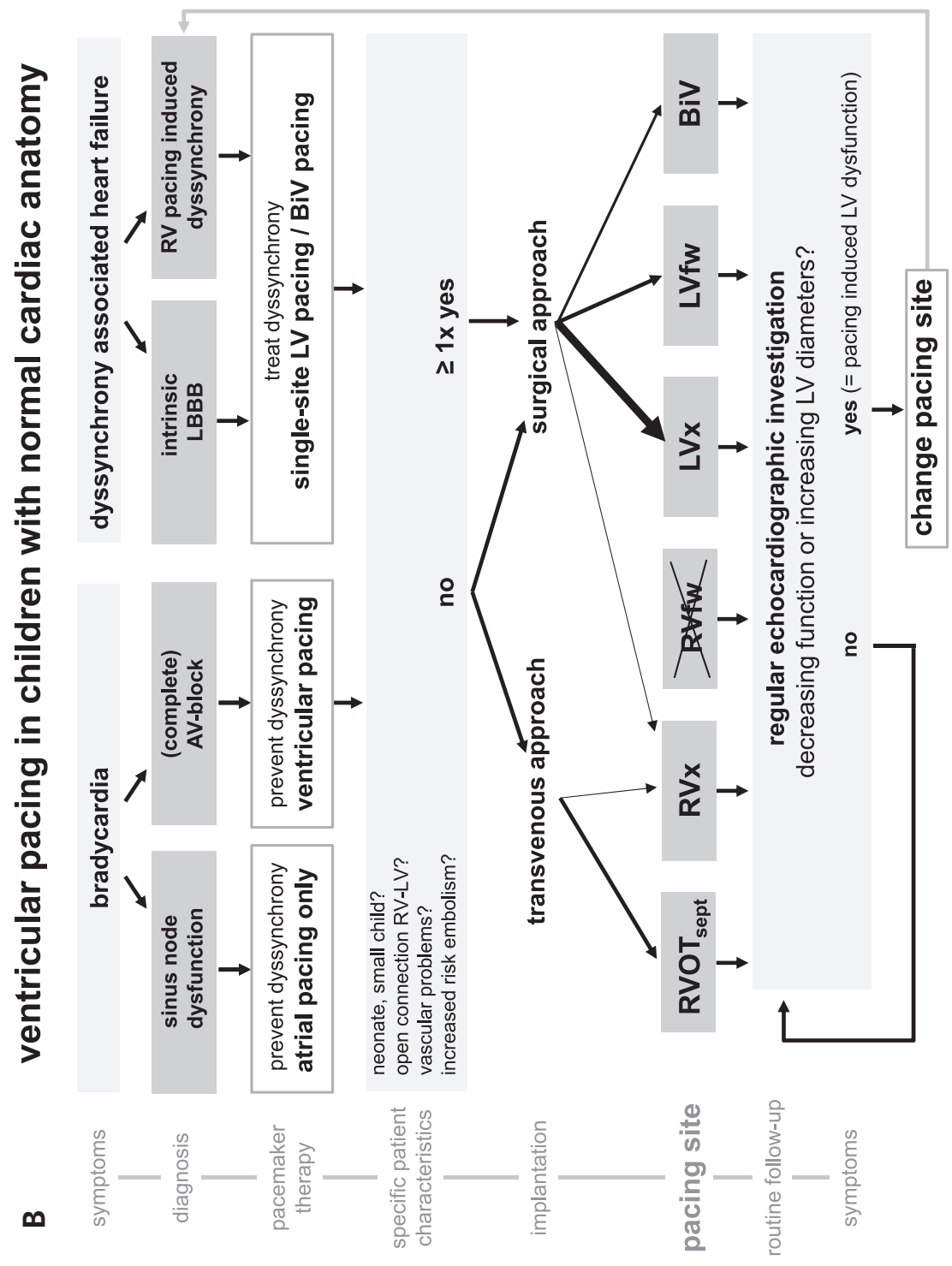


In children with abnormal cardiac anatomy, influences of different pacing-sites on cardiac function are less well known. Based on rationale and recent research (chapter 8), we suggest implantation of the lead for chronic ventricular pacing preferably at the systemic ventricle (Figure 3B). However, in patients with abnormal cardiac anatomy, it may currently be more correct to investigate various pacing-sites for their effects on electrical activation and cardiac function, and to implant the electrode at the site associated with optimal electrical activation pattern and cardiac function.

In general, in the absence of intrinsic dyssynchrony, the systemic ventricle offers the sites where pacing results in least mechanical dyssynchrony and best cardiac function and should therefore be considered when chronic ventricular pacing is indicated. Both single-site LV pacing and BiV pacing may induce reversal of remodeling and reversal of the impaired LV function in deteriorated hearts after pacing. Changing the site of pacing to either BiV or single-site pacing at the systemic ventricle should be considered as soon as echocardiography reveals signs of ventricular dilatation or dysfunction. Regular echocardiographic checkup is therefore warranted in all (pediatric) patients with pacemaker therapy, and especially in children who are paced at the non-systemic ventricle. 


\section{REFERENCES}

1. Epstein $A E$, DiMarco JP, Ellenbogen KA, Estes NA, 3rd, Freedman RA, Gettes LS, Gillinov AM, Gregoratos G, Hammill SC, Hayes DL, Hlatky MA, Newby LK, Page RL, Schoenfeld MH, Silka MJ, Stevenson LW, Sweeney MO, Smith SC, Jr., Jacobs AK, Adams CD, Anderson JL, Buller CE, Creager MA, Ettinger SM, Faxon DP, Halperin JL, Hiratzka LF, Hunt SA, Krumholz HM, Kushner FG, Lytle BW, Nishimura RA, Ornato JP, Page RL, Riegel B, Tarkington LG, Yancy CW. ACC/AHA/HRS 2008 Guidelines for Device-Based Therapy of Cardiac Rhythm Abnormalities: a report of the American College of Cardiology/American Heart Association Task Force on Practice Guidelines (Writing Committee to Revise the ACC/AHA/NASPE 2002 Guideline Update for Implantation of Cardiac Pacemakers and Antiarrhythmia Devices) developed in collaboration with the American Association for Thoracic Surgery and Society of Thoracic Surgeons. I Am Coll Cardiol 2008; 51(21):e1-62.

2. Mullens W, Grimm RA, Verga T, Dresing T, Starling RC, Wilkoff BL, Tang WH. Insights from a cardiac resynchronization optimization clinic as part of a heart failure disease management program. J Am Coll Cardiol 2009; 53(9):765-773.

3. Zareba W, Klein H, Cygankiewicz I, Hall WJ, McNitt S, Brown M, Cannom D, Daubert JP, Eldar M, Gold MR, Goldberger JJ, Goldenberg I, Lichstein E, Pitschner H, Rashtian M, Solomon S, Viskin S, Wang P, Moss AJ. Effectiveness of Cardiac Resynchronization Therapy by QRS Morphology in the Multicenter Automatic Defibrillator Implantation Trial-Cardiac Resynchronization Therapy (MADITCRT). Circulation 2011; 123(10):1061-1072.

4. Linde C, Abraham WT, Gold MR, Daubert C. Cardiac resynchronization therapy in asymptomatic or mildly symptomatic heart failure patients in relation to etiology: results from the REVERSE (REsynchronization reVErses Remodeling in Systolic Left vEntricular Dysfunction) study. J Am Coll Cardiol 2010; 56(22):1826-1831.

5. Delgado V, van Bommel RJ, Bertini M, Borleffs CJ, Marsan NA, Arnold CT, Nucifora G, van de Veire NR, Ypenburg C, Boersma E, Holman ER, Schalij MJ, Bax JJ. Relative merits of left ventricular dyssynchrony, left ventricular lead position, and myocardial scar to predict long-term survival of ischemic heart failure patients undergoing cardiac resynchronization therapy. Circulation 2011; 123(1):70-78.

6. Chung ES, Leon AR, Tavazzi L, Sun JP, Nihoyannopoulos P, Merlino J, Abraham WT, Ghio S, Leclercq C, Bax JJ, Yu CM, Gorcsan J, 3rd, St John Sutton M, De Sutter J, Murillo J. Results of the Predictors of Response to CRT (PROSPECT) trial. Circulation 2008; 117(20):2608-2616.

7. Seo Y, Ito H, Nakatani S, Takami M, Naito S, Shiga T, Ando K, Wakayama Y, Aonuma K. The role of echocardiography in predicting responders to cardiac resynchronization therapy. Circ J 2011; 75(5):1156-1163. 
8. Witte KK, Pipes RR, Nanthakumar K, Parker JD. Biventricular pacemaker upgrade in previously paced heart failure patients_improvements in ventricular dyssynchrony. J Card Fail 2006; 12(3):199-204.

9. Leclercq C, Cazeau S, Lellouche D, Fossati F, Anselme F, Davy JM, Sadoul N, Klug D, Mollo L, Daubert JC. Upgrading from single chamber right ventricular to biventricular pacing in permanently paced patients with worsening heart failure: The RD-CHF Study. Pacing Clin Electrophysiol 2007; 30 Suppl 1:S23-30.

10. Laurenzi F, Achilli A, Avella A, Peraldo C, Orazi S, Perego GB, Cesario A, Valsecchi S, De Santo T, Puglisi $A$, Tondo $C$. Biventricular upgrading in patients with conventional pacing system and congestive heart failure: results and response predictors. Pacing Clin Electrophysiol 2007; 30(9):1096-1104.

11. Shimano M, Tsuji Y, Yoshida Y, Inden Y, Tsuboi N, Itoh T, Suzuki H, Muramatsu T, Okada T, Harata S, Yamada T, Hirayama H, Nattel S, Murohara T. Acute and chronic effects of cardiac resynchronization in patients developing heart failure with long-term pacemaker therapy for acquired complete atrioventricular block. Europace 2007; 9(10):869-874.

12. Delnoy PP, Ottervanger JP, Luttikhuis HO, Elvan A, Misier AR, Beukema WP, van Hemel NM. Longterm clinical response of cardiac resynchronization after chronic right ventricular pacing. Am J Cardiol 2009; 104(1):116-121.

13. Linde C, Abraham WT, Gold MR, St John Sutton M, Ghio S, Daubert C. Randomized trial of cardiac resynchronization in mildly symptomatic heart failure patients and in asymptomatic patients with left ventricular dysfunction and previous heart failure symptoms. I Am Coll Cardiol 2008; 52(23):1834-1843.

14. Moss AJ, Hall WJ, Cannom DS, Klein H, Brown MW, Daubert JP, Estes NA, 3rd, Foster E, Greenberg H, Higgins SL, Pfeffer MA, Solomon SD, Wilber D, Zareba W. Cardiac-resynchronization therapy for the prevention of heart-failure events. N Eng J Med 2009; 361(14):1329-1338.

15. van Geldorp IE, Vernooy K, Delhaas T, Prins MH, Crijns HJ, Prinzen FW, Dijkman B. Beneficial effects of biventricular pacing in chronically right ventricular paced patients with mild cardiomyopathy. Europace 2010; 12(2):223-229.

16. Daubert C, Gold MR, Abraham WT, Ghio S, Hassager C, Goode G, Szili-Torok T, Linde C. Prevention of disease progression by cardiac resynchronization therapy in patients with asymptomatic or mildly symptomatic left ventricular dysfunction: insights from the European cohort of the REVERSE (Resynchronization Reverses Remodeling in Systolic Left Ventricular Dysfunction) trial. J Am Coll Cardiol 2009; 54(20):1837-1846.

17. Auricchio A, Stellbrink C, Block M, Sack S, Vogt J, Bakker P, Klein H, Kramer A, Ding J, Salo R, Tockman B, Pochet T, Spinelli J. Effect of pacing chamber and atrioventricular delay on acute systolic function of paced patients with congestive heart failure. The Pacing Therapies for Congestive Heart Failure Study Group. The Guidant Congestive Heart Failure Research Group. Circulation 1999; 99(23):2993-3001. 
18. Auricchio A, Stellbrink C, Sack S, Block M, Vogt J, Bakker P, Huth C, Schondube F, Wolfhard U, Bocker D, Krahnefeld O, Kirkels $\mathrm{H}$. Long-term clinical effect of hemodynamically optimized cardiac resynchronization therapy in patients with heart failure and ventricular conduction delay. J Am Coll Cardiol 2002; 39(12):2026-2033.

19. Perego GB, Chianca R, Facchini M, Frattola A, Balla E, Zucchi S, Cavaglia S, Vicini I, Negretto M, Osculati G. Simultaneous vs. sequential biventricular pacing in dilated cardiomyopathy: an acute hemodynamic study. Eur J Heart Fail 2003; 5(3):305-313.

20. Whinnett ZI, Davies JE, Willson K, Manisty CH, Chow AW, Foale RA, Davies DW, Hughes AD, Mayet J, Francis DP. Haemodynamic effects of changes in atrioventricular and interventricular delay in cardiac resynchronisation therapy show a consistent pattern: analysis of shape, magnitude and relative importance of atrioventricular and interventricular delay. Heart 2006; 92(11):16281634.

21. Pabari PA, Willson K, Stegemann B, van Geldorp IE, Kyriacou A, Moraldo M, Mayet J, Hughes AD, Francis DP. When is an optimization not an optimization? Evaluation of clinical implications of information content (signal-to-noise ratio) in optimization of cardiac resynchronization therapy, and how to measure and maximize it. Heart Fail Rev 2011; 16(3):277-290.

22. Ritter P, Dib J, Lelievre T. Quick determination of the optimal AV delay at rest in patients paced in DDD mode for complete AV block. Eur J Cardiac Pacing Electrophysiol 1994; 4(2):A163.

23. Cleland JG, Daubert JC, Erdmann E, Freemantle N, Gras D, Kappenberger L, Tavazzi L. The effect of cardiac resynchronization on morbidity and mortality in heart failure. N Engl J Med 2005; 352(15):1539-1549.

24. Whinnett ZI, Davies JE, Willson K, Chow AW, Foale RA, Davies DW, Hughes AD, Francis DP, Mayet J. Determination of optimal atrioventricular delay for cardiac resynchronization therapy using acute non-invasive blood pressure. Europace 2006; 8(5):358-366.

25. Gras D, Gupta MS, Boulogne E, Guzzo L, Abraham WT. Optimization of AV and VV delays in the real-world CRT patient population: an international survey on current clinical practice. Pacing Clin Electrophysiol 2009; 32 Suppl 1:S236-239.

26. van Geldorp IE, Delhaas T, Hermans B, Vernooy K, Broers B, Klimusina J, Regoli F, Faletra FF, Moccetti T, Gerritse B, Cornelussen R, Settels JJ, Crijns HJ, Auricchio A, Prinzen FW. Comparison of a noninvasive arterial pulse contour technique and echo Doppler aorta velocity-time integral on stroke volume changes in optimization of cardiac resynchronization therapy. Europace 2011; 13(1):87-95.

27. Scharf C, Li P, Muntwyler J, Chugh A, Oral H, Pelosi F, Morady F, Armstrong WF. Rate-dependent AV delay optimization in cardiac resynchronization therapy. Pacing Clin Electrophysiol 2005; 28(4):279-284.

28. Valzania C, Eriksson MJ, Boriani G, Gadler F. Cardiac resynchronization therapy during rest and exercise: comparison of two optimization methods. Europace 2008; 10(10):1161-1169. 
29. Derval N, Steendijk P, Gula L, Deplagne A, Laborderie J, Sacher F, Knecht S, Wright M, Nault I, Ploux S, Ritter P, Bordachar P, Lafitte S, Reant P, Klein GJ, Narayan SM, Garrigue S, Hocini M, Haissaguerre M, Clementy J, Jais P. Optimizing hemodynamics in heart failure patients by systematic screening of left ventricular pacing sites: the lateral left ventricular wall and the coronary sinus are rarely the best sites. J Am Coll Cardiol 2010; 55(6):566-575.

30. Spragg DD, Dong J, Fetics BJ, Helm R, Marine JE, Cheng A, Henrikson CA, Kass DA, Berger RD. Optimal left ventricular endocardial pacing sites for cardiac resynchronization therapy in patients with ischemic cardiomyopathy. J Am Coll Cardiol 2010; 56(10):774-781.

31. Rademakers LM, Vankerckhoven R, Vandeursen CJ, Strik M, Vanhunnik A, Kuiper M, Lampert A, Klersy C, Leyva F, Auricchio A, Maessen JG, Prinzen FW. Myocardial Infarction Does Not Preclude Electrical and Hemodynamic Benefits of CRT in Dyssynchronous Canine Hearts. Circ Arrhythm Electrophysiol 2010.

32. Becker M, Zwicker C, Kaminski M, Napp A, Altiok E, Ocklenburg C, Friedman Z, Adam D, Schauerte P, Marx N, Hoffmann R. Dependency of cardiac resynchronization therapy on myocardial viability at the LV lead position. JACC Cardiovasc Imaging 2011; 4(4):366-374.

33. Thambo JB, Bordachar P, Garrigue S, Lafitte $S$, Sanders $P$, Reuter $S$, Girardot R, Crepin D, Reant $P$, Roudaut R, Jais $P$, Haissaguerre M, Clementy J, Jimenez M. Detrimental ventricular remodeling in patients with congenital complete heart block and chronic right ventricular apical pacing. Circulation 2004; 110(25):3766-3772.

34. Karpawich PP. Chronic right ventricular pacing and cardiac performance: the pediatric perspective. Pacing Clin Electrophysiol 2004; 27 (6 Pt 2):844-849.

35. Janousek J, Tomek V, Chaloupecky V, Gebauer RA. Dilated cardiomyopathy associated with dualchamber pacing in infants: improvement through either left ventricular cardiac resynchronization or programming the pacemaker off allowing intrinsic normal conduction. I Cardiovasc Electrophysiol 2004; 15(4):470-474.

36. Deshmukh P, Casavant DA, Romanyshyn M, Anderson K. Permanent, direct His-bundle pacing: a novel approach to cardiac pacing in patients with normal His-Purkinje activation. Circulation 2000; 101(8):869-877.

37. Deshmukh PM, Romanyshyn M. Direct His-bundle pacing: present and future. Pacing Clin Electrophysiol 2004; 27(6 Pt 2):862-870.

38. Zanon F, Baracca E, Aggio S, Pastore G, Boaretto G, Cardano P, Marotta T, Rigatelli G, Galasso M, Carraro M, Zonzin P. A feasible approach for direct his-bundle pacing using a new steerable catheter to facilitate precise lead placement. J Cardiovasc Electrophysiol 2006; 17(1):29-33.

39. Hillock RJ, Stevenson IH, Mond HG. The right ventricular outflow tract: a comparative study of septal, anterior wall, and free wall pacing. Pacing Clin Electrophysiol 2007; 30(8):942-947.

40. Mond HG, Hillock RJ, Stevenson IH, McGavigan AD. The right ventricular outflow tract: the road to septal pacing. Pacing Clin Electrophysiol 2007; 30(4):482-491. 
41. de Cock CC, Giudici MC, Twisk JW. Comparison of the haemodynamic effects of right ventricular outflow-tract pacing with right ventricular apex pacing: a quantitative review. Europace 2003; 5(3):275-278.

42. Albouaini K, Alkarmi A, Mudawi T, Gammage MD, Wright DJ. Selective site right ventricular pacing. Heart 2009; 95(24):2030-2039.

43. Cowell R, Morris-Thurgood J, Ilsley C, Paul V. Septal short atrioventricular delay pacing: additional hemodynamic improvements in heart failure. Pacing Clin Electrophysiol 1994; 17(11 Pt 2):1980-1983.

44. Karpawich PP, Mital S. Comparative left ventricular function following atrial, septal, and apical single chamber heart pacing in the young. Pacing Clin Electrophysio/ 1997; 20(8 Pt 1):1983-1988.

45. Mera F, DeLurgio DB, Patterson RE, Merlino JD, Wade ME, Leon AR. A comparison of ventricular function during high right ventricular septal and apical pacing after his-bundle ablation for refractory atrial fibrillation. Pacing Clin Electrophysiol 1999; 22(8):1234-1239.

46. Schwaab B, Frohlig G, Alexander C, Kindermann M, Hellwig N, Schwerdt H, Kirsch CM, Schieffer $\mathrm{H}$. Influence of right ventricular stimulation site on left ventricular function in atrial synchronous ventricular pacing. J Am Coll Cardiol 1999; 33(2):317-323.

47. Tse HF, Yu C, Wong KK, Tsang V, Leung YL, Ho WY, Lau CP. Functional abnormalities in patients with permanent right ventricular pacing: the effect of sites of electrical stimulation. J Am Coll Cardiol 2002; 40(8):1451-1458.

48. Victor F, Mabo P, Mansour H, Pavin D, Kabalu G, de Place C, Leclercq C, Daubert JC. A randomized comparison of permanent septal versus apical right ventricular pacing: short-term results. J Cardiovasc Electrophysio/ 2006; 17(3):238-242.

49. Occhetta E, Bortnik M, Magnani A, Francalacci G, Piccinino C, Plebani L, Marino P. Prevention of ventricular desynchronization by permanent para-Hisian pacing after atrioventricular node ablation in chronic atrial fibrillation: a crossover, blinded, randomized study versus apical right ventricular pacing. J Am Coll Cardiol 2006; 47(10):1938-1945.

50. Dubin AM, Janousek J, Rhee E, Strieper MJ, Cecchin F, Law IH, Shannon KM, Temple J, Rosenthal E, Zimmerman FJ, Davis A, Karpawich PP, Al Ahmad A, Vetter VL, Kertesz NJ, Shah M, Snyder C, Stephenson E, Emmel M, Sanatani S, Kanter R, Batra A, Collins KK. Resynchronization therapy in pediatric and congenital heart disease patients: an international multicenter study. J Am Coll Cardiol 2005; 46(12):2277-2283.

51. Janousek J, Gebauer RA, Abdul-Khaliq H, Turner M, Kornyei L, Grollmuss O, Rosenthal E, Villain E, Fruh A, Paul T, Blom NA, Happonen JM, Bauersfeld U, Jacobsen JR, van den Heuvel F, Delhaas T, Papagiannis J, Trigo C. Cardiac resynchronisation therapy in paediatric and congenital heart disease: differential effects in various anatomical and functional substrates. Heart 2009; 95(14):1165-1171.

52. Janousek J, Gebauer RA. Cardiac resynchronization therapy in pediatric and congenital heart disease. Pacing Clin Electrophysiol 2008; 31 Suppl 1:S21-23.

53. Vanagt WY, Prinzen FW, Delhaas T. Reversal of pacing-induced heart failure by left ventricular apical pacing. N Engl J Med 2007; 357(25):2637-2638. 
54. Tomaske M, Breithardt OA, Balmer C, Bauersfeld U. Successful cardiac resynchronization with single-site left ventricular pacing in children. Int J Cardiol 2009; 136(2):136-143.

55. Auricchio A. Pacing the left ventricle: does underlying rhythm matter? J Am Coll Cardiol 2004; 43(2):239-240.

56. Etienne Y, Mansourati J, Gilard M, Valls-Bertault V, Boschat J, Benditt DG, Lurie KG, Blanc JJ. Evaluation of left ventricular based pacing in patients with congestive heart failure and atrial fibrillation. Am J Cardiol 1999; 83(7):1138-1140, A1139.

57. Touiza A, Etienne Y, Gilard M, Fatemi M, Mansourati J, Blanc JJ. Long-term left ventricular pacing: assessment and comparison with biventricular pacing in patients with severe congestive heart failure. J Am Coll Cardiol 2001; 38(7):1966-1970.

58. Mills RW, Cornelussen RN, Mulligan LJ, Strik M, Rademakers LM, Skadsberg ND, van Hunnik A, Kuiper M, Lampert A, Delhaas T, Prinzen FW. Left ventricular septal and left ventricular apical pacing chronically maintain cardiac contractile coordination, pump function and efficiency. Circ Arrhythm Electrophysiol 2009; 2(5):571-579.

59. Vanagt WY, Verbeek XA, Delhaas T, Mertens L, Daenen WJ, Prinzen FW. The left ventricular apex is the optimal site for pediatric pacing: correlation with animal experience. Pacing Clin Electrophysiol 2004; 27(6 Pt 2):837-843.

60. van Geldorp IE, Vanagt WY, Bauersfeld U, Tomaske M, Prinzen FW, Delhaas T. Chronic left ventricular pacing preserves left ventricular function in children. Pediatr Cardio/ 2009; 30(2):125-132.

61. Tomaske M, Breithardt OA, Bauersfeld U. Preserved cardiac synchrony and function with singlesite left ventricular epicardial pacing during mid-term follow-up in paediatric patients. Europace 2009; 11(9):1168-1176.

62. van Geldorp IE, Delhaas T, Gebauer RA, Frias P, Tomaske M, Friedberg MK, Tisma-Dupanovic S, Elders J, Fruh A, Gabbarini F, Kubus P, Illikova V, Tsao S, Blank AC, Hiippala A, Sluysmans T, Karpawich P, Clur SA, Ganame X, Collins KK, Dann G, Thambo JB, Trigo C, Nagel B, Papagiannis J, Rackowitz A, Marek J, Nurnberg JH, Vanagt WY, Prinzen FW, Janousek J. Impact of the permanent ventricular pacing site on left ventricular function in children: a retrospective multicentre survey. Heart 2011; 97(24):2051-2055.

63. Janousek J, van Geldorp IE, Krupickova S, Rosenthal E, Tomaske M, Fruh A, Elders J, Hiippala A, Kerst G, Gebauer RA, Kubus P, Frias P, Gabbarini F, Clur SA, Nagel B, Ganame X, Papagiannis J, Marek J, Tisma-Dupanovic S, Tsao S, Nurnberg JH, Wren C, Friedberg MK, De Guillebon M, Prinzen FW, Delhaas T. Permanent Cardiac Pacing in Children - Choosing the Optimal Pacing-site: A MultiCenter Study. submitted 2012.

64. Janousek J, van Geldorp IE, Prinzen FW, Vanagt WY, Elders J, Fruh A, Kubus P, Tomaske M, Ganame J, Nagel B, Papagiannis J, Wren C, Nurnberg JH, Delhaas T, Gebauer RA. LV Pacing Preserves Ventricular Synchrony and Function in Children With AV Block and Structurally Normal Heart as Compared to RV Pacing Sites: a Multi-Center Study. Heart Rhythm 2010; 7(S5). 
65. Jaeggi ET, Hamilton RM, Silverman ED, Zamora SA, Hornberger LK. Outcome of children with fetal, neonatal or childhood diagnosis of isolated congenital atrioventricular block. A single institution's experience of 30 years. J Am Coll Cardiol 2002; 39(1):130-137.

66. Nield LE, Silverman ED, Smallhorn JF, Taylor GP, Mullen JB, Benson LN, Hornberger LK. Endocardial fibroelastosis associated with maternal anti-Ro and anti-La antibodies in the absence of atrioventricular block. J Am Coll Cardiol 2002; 40(4):796-802.

67. Villain E, Coastedoat-Chalumeau N, Marijon E, Boudjemline Y, Piette JC, Bonnet D. Presentation and prognosis of complete atrioventricular block in childhood, according to maternal antibody status. J Am Coll Cardiol 2006; 48(8):1682-1687.

68. Chow PC, Liang XC, Lam WW, Cheung EW, Wong KT, Cheung YF. Mechanical right ventricular dyssynchrony in patients after atrial switch operation for transposition of the great arteries. Am J Cardiol 2008; 101(6):874-881.

69. Huhta JC, Maloney JD, Ritter DG, Ilstrup DM, Feldt RH. Complete atrioventricular block in patients with atrioventricular discordance. Circulation 1983; 67(6):1374-1377.

70. Lumens J, Arts T, Broers B, Boomars KA, van Paassen P, Prinzen FW, Delhaas T. Right ventricular free wall pacing improves cardiac pump function in severe pulmonary arterial hypertension: a computer simulation analysis. Am J Physiol Heart Circ Physiol 2009; 297(6):H2196-2205.

71. Pettersen E, Helle-Valle T, Edvardsen T, Lindberg H, Smith HJ, Smevik B, Smiseth OA, Andersen K. Contraction pattern of the systemic right ventricle shift from longitudinal to circumferential shortening and absent global ventricular torsion. J Am Coll Cardiol 2007; 49(25):2450-2456.

72. Dubin AM, Feinstein JA, Reddy VM, Hanley FL, Van Hare GF, Rosenthal DN. Electrical resynchronization: a novel therapy for the failing right ventricle. Circulation 2003; 107(18):22872289.

73. Stephenson EA, Cecchin F, Alexander ME, Triedman JK, Walsh EP, Berul Cl. Relation of right ventricular pacing in tetralogy of Fallot to electrical resynchronization. Am J Cardiol 2004; 93(11):1449-1452, A1412.

74. Byrne MJ, Helm RH, Daya S, Osman NF, Halperin HR, Berger RD, Kass DA, Lardo AC. Diminished left ventricular dyssynchrony and impact of resynchronization in failing hearts with right versus left bundle branch block. J Am Coll Cardiol 2007; 50(15):1484-1490.

75. Bordachar P, Iriart X, Chabaneix J, Sacher F, Lafitte S, Jais P, Haissaguerre M, Clementy J, Dos Santos P, Thambo JB. Presence of ventricular dyssynchrony and haemodynamic impact of right ventricular pacing in adults with repaired Tetralogy of Fallot and right bundle branch block. Europace 2008; 10(8):967-971.

76. Thambo JB, Dos Santos P, De Guillebon M, Roubertie F, Labrousse L, Sacher F, Iriart X, Lafitte $S$, Ploux S, Jais P, Roques X, Haissaguerre M, Ritter P, Clementy J, Narayan SM, Bordachar P. Biventricular stimulation improves right and left ventricular function after tetralogy of Fallot repair: acute animal and clinical studies. Heart Rhythm 2010; 7(3):344-350.

77. Delhaas T, Prinzen FW. Right ventricular or biventricular pacing in repaired tetralogy of Fallot? Heart Rhythm 2010; 7(3):351-352. 
78. Thambo JB, De Guillebon M, Dos Santos P, Xhaet O, Ploux S, Iriart X, Haissaguerre M, Bordachar P. Electrical dyssynchrony and resynchronization in tetralogy of Fallot. Heart Rhythm 2011; 8(6):909-914.

79. Horovitz A, De Guillebon M, van Geldorp IE, Bordachar P, Roubertie F, Iriart X, Douard H, Haissaguerre $M$, Thambo JB. Effects of non-systemic ventricular pacing in patients with transposition of the great arteries and atrial redirection. J Cardiovasc Electrophysiol 2012.

80. Prinzen FW, Peschar M. Relation between the pacing induced sequence of activation and left ventricular pump function in animals. Pacing Clin Electrophysio/ 2002; 25(4 Pt 1):484-498.

81. Puggioni E, Brignole M, Gammage M, Soldati E, Bongiorni MG, Simantirakis EN, Vardas P, Gadler F, Bergfeldt L, Tomasi C, Musso G, Gasparini G, Del Rosso A. Acute comparative effect of right and left ventricular pacing in patients with permanent atrial fibrillation. J Am Coll Cardiol 2004; 43(2):234-238.

82. Little WC, Reeves RC, Arciniegas J, Katholi RE, Rogers EW. Mechanism of abnormal interventricular septal motion during delayed left ventricular activation. Circulation 1982; 65(7):1486-1491.

83. Wyman BT, Hunter WC, Prinzen FW, Faris OP, McVeigh ER. Effects of single- and biventricular pacing on temporal and spatial dynamics of ventricular contraction. Am J Physiol Heart Circ Physiol 2002; 282(1):H372-379.

84. Peschar M, de Swart H, Michels KJ, Reneman RS, Prinzen FW. Left ventricular septal and apex pacing for optimal pump function in canine hearts. J Am Coll Cardiol 2003; 41(7):1218-1226.

85. Gebauer RA, Tomek V, Kubus P, Razek V, Matejka T, Salameh A, Kostelka M, Janousek J. Differential effects of the site of permanent epicardial pacing on left ventricular synchrony and function in the young: implications for lead placement. Europace 2009; 11(12):1654-1659.

86. Dodge-Khatami A, Kadner A, Dave H, Rahn M, Pretre R, Bauersfeld U. Left heart atrial and ventricular epicardial pacing through a left lateral thoracotomy in children: a safe approach with excellent functional and cosmetic results. Eur J Cardiothorac Surg 2005; 28(4):541-545.

87. Prinzen FW, Van Oosterhout MF, Vanagt WY, Storm C, Reneman RS. Optimization of ventricular function by improving the activation sequence during ventricular pacing. Pacing Clin Electrophysiol 1998; 21(11 Pt 2):2256-2260.

88. Zimmerman FJ, Starr JP, Koenig PR, Smith P, Hijazi ZM, Bacha EA. Acute hemodynamic benefit of multisite ventricular pacing after congenital heart surgery. Ann Thorac Surg 2003; 75(6):17751780.

89. Ramanathan C, Ghanem RN, Jia P, Ryu K, Rudy Y. Noninvasive electrocardiographic imaging for cardiac electrophysiology and arrhythmia. Nat Med 2004; 10(4):422-428.

90. Silva JN, Ghosh S, Bowman TM, Rhee EK, Woodard PK, Rudy Y. Cardiac resynchronization therapy in pediatric congenital heart disease: insights from noninvasive electrocardiographic imaging. Heart Rhythm 2009; 6(8):1178-1185. 
91. van Deursen C, van Geldorp IE, Rademakers LM, van Hunnik A, Kuiper M, Klersy C, Auricchio A, Prinzen FW. Left ventricular endocardial pacing improves resynchronization therapy in canine left bundle-branch hearts. Circ Arrhythm Electrophysiol 2009; 2(5):580-587.

92. Bordachar P, Derval N, Ploux S, Garrigue S, Ritter P, Haissaguerre M, Jais P. Left ventricular endocardial stimulation for severe heart failure. J Am Coll Cardiol 2010; 56(10):747-753.

93. Echt DS, Cowan MW, Riley RE, Brisken AF. Feasibility and safety of a novel technology for pacing without leads. Heart Rhythm 2006; 3(10):1202-1206.

94. Lee KL, Tse HF, Echt DS, Lau CP. Temporary leadless pacing in heart failure patients with ultrasoundmediated stimulation energy and effects on the acoustic window. Heart Rhythm 2009; 6(6):742-748.

95. Wieneke $\mathrm{H}$, Konorza $\mathrm{T}$, Erbel $\mathrm{R}$, Kisker E. Leadless pacing of the heart using induction technology: a feasibility study. Pacing Clin Electrophysiol 2009; 32(2):177-183.

96. van Geldorp IE, Vanagt WY, Prinzen FW, Delhaas T. Chronic ventricular pacing in children: toward prevention of pacing-induced heart disease. Heart Fail Rev 2011; 16(3):305-314.

97. Nielsen JC, Kristensen L, Andersen HR, Mortensen PT, Pedersen OL, Pedersen AK. A randomized comparison of atrial and dual-chamber pacing in 177 consecutive patients with sick sinus syndrome: echocardiographic and clinical outcome. J Am Coll Cardiol 2003; 42(4):614-623.

98. Andersen HR, Nielsen JC, Thomsen PE, Thuesen L, Mortensen PT, Vesterlund T, Pedersen AK. Long-term follow-up of patients from a randomised trial of atrial versus ventricular pacing for sick-sinus syndrome. Lancet 1997; 350(9086):1210-1216.

99. Batra AS, Wells WJ, Hinoki KW, Stanton RA, Silka MJ. Late recovery of atrioventricular conduction after pacemaker implantation for complete heart block associated with surgery for congenital heart disease. J Thorac Cardiovasc Surg 2003; 125(6):1291-1293.

100. Vanagt WY, Verbeek XA, Delhaas T, Gewillig M, Mertens L, Wouters P, Meyns B, Daenen WJ, Prinzen FW. Acute hemodynamic benefit of left ventricular apex pacing in children. Ann Thorac Surg 2005; 79(3):932-936.

101. Gebauer RA, Tomek V, Salameh A, Marek J, Chaloupecky V, Gebauer R, Matejka T, Vojtovic P, Janousek J. Predictors of left ventricular remodelling and failure in right ventricular pacing in the young. Eur Heart J 2009; 30(9):1097-1104.

102. Vatasescu R, Shalganov T, Paprika D, Kornyei L, Prodan Z, Bodor G, Szatmari A, Szili-Torok T. Evolution of left ventricular function in paediatric patients with permanent right ventricular pacing for isolated congenital heart block: a medium term follow-up. Europace 2007; 9(4):228-232.

103. Shalganov TN, Paprika D, Vatasescu R, Kardos A, Mihalcz A, Kornyei L, Szatmari A, Szili-Torok T. Mid-term echocardiographic follow up of left ventricular function with permanent right ventricular pacing in pediatric patients with and without structural heart disease. Cardiovasc Ultrasound 2007; 5:13. 



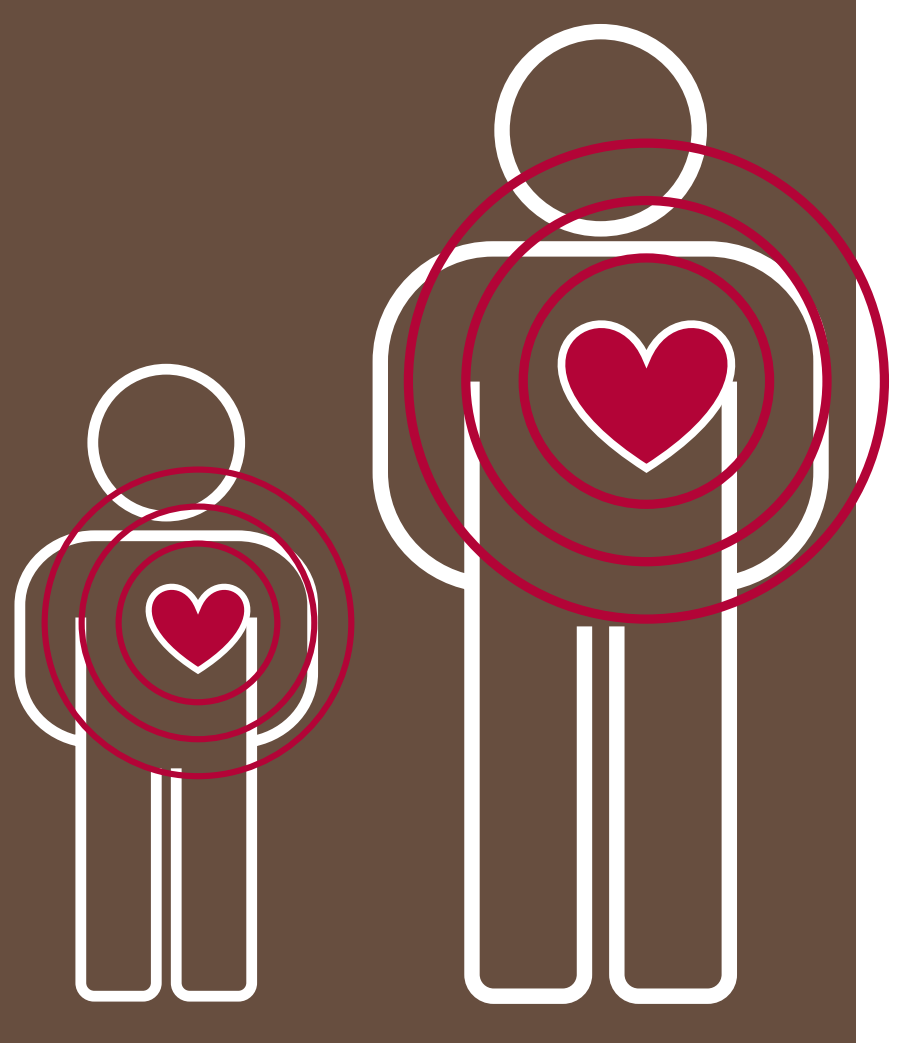

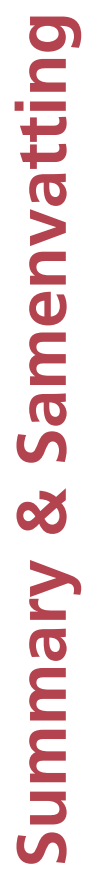




\section{SUMMARY}

\section{Improving ventricular pacing in adults and children to treat or to avoid dyssynchrony-induced cardiac failure}

The rationales for the general aim and the studies presented in this thesis are introduced in chapter 1 and further elucidated in chapter 2 . The common background of the research presented in this thesis is that disturbances in the physiological pattern of electrical ventricular activation, either due to a partially disrupted conduction system or by pacing of the ventricle, may be accompanied by dyssynchronous contraction of the left ventricular (LV) myocardium. Both intrinsic left bundle branch block and conventional right ventricular (RV) pacing are associated with functional impairment and structural remodeling of the LV, as well as with an increased risk for heart failure.

Cardiac resynchronization therapy (CRT) by means of biventricular (BiV) pacing is on average beneficial in patients with severe LV dysfunction and severe clinical heart failure associated with either intrinsic or pacing-induced dyssynchrony. However, the individual response to CRT varies widely between patients, with inadequate response in $30-50 \%$ of the patients receiving CRT. Our research on optimization of CRT in adult patients to improve treatment of dyssynchrony-induced cardiac failure is presented in chapters 3 and 4.

In patients with congenital or acquired complete atrioventricular (AV) block, ventricular pacing is indicated when the intrinsic rhythm of the ventricles is inadequate. The awareness of the potentially deleterious effects of conventional RV pacing has initiated the search for alternative approaches for chronic ventricular pacing. The general objective of the studies presented in chapters 5-8 was to define better pacing strategies in the young, aiming at prevention of pacing-induced cardiac deterioration.

\section{Upgrade to biventricular pacing may also be considered in chronically paced patients with relatively mild cardiomyopathy}

By restoration of a more coordinated contraction pattern, CRT aims to reverse the deleterious effects that may originate from LV dyssynchrony. It seems therefore reasonable to suppose that all patients with LV dyssynchrony may benefit from resynchronization, irrespective of the level of heart failure or etiology of dyssynchrony. In the study presented in chapter 3, we compared the effects of BiV pacing and RV pacing in previously chronically RV paced patients with relatively mild cardiomyopathy (LV ejection fraction 36 $10 \%$ ) and, hence, no established indication for CRT. Biventricular pacing resulted in better LV function, less LV dilatation and less clinical symptoms. Furthermore, the response to BiV pacing did not correlate to the severity of either LV dysfunction or LV remodeling. Hence, upgrade to BiV pacing should be considered in chronically paced patients with relatively mild cardiomyopathy, in whom it may serve to prevent rather than to treat heart failure. 


\section{In the optimization of cardiac resynchronization, assessment of stroke volume changes using a non-invasive arterial pulse contour technique is promising}

Hemodynamic and long-term benefits brought by CRT may be improved by tailoring of devicesettings to the individual patient. However, procedures to optimize device-settings, as well as measurements to guide these optimization procedures, are still under debate. We sought for a patient-friendly non-invasive measurement to guide optimization procedures. Nexfin CO-Trek ${ }^{\circledR}$ (BMEYE B.V., Amsterdam, Netherlands) is such a non-invasive system, as it uses a finger-cuff with an arterial pulse contour technique for continuous measurement of stroke volume. In the study presented in chapter 4, we investigated the accuracy and feasibility of this non-invasive arterial pulse contour technique in optimization of AV delay in CRT, through comparisons with echo Doppler aortic velocity-time integral at 1) the measurement of beat-to-beat stroke volume changes, 2) the assessment of relative effects of CRT at various AV delays, and 3) the determination of an optimal AV delay. Nexfin $\mathrm{CO}^{-}$Trek $^{\circledR}$ had a good precision at all three points. Therefore, and because of its easy and patient-friendly use, we judged the non-invasive arterial pulse contour technique as a promising tool to evaluate hemodynamic changes for individualized optimization of CRT.

\section{In children with isolated atrioventricular block, chronic left ventricular pacing, as compared with right ventricular pacing, results in a better left ventricular function}

Given the acute beneficial hemodynamic effects of single-site LV pacing shown by earlier studies of our group, we investigated whether chronic LV pacing is superior to RV pacing in children with AV block and structurally normal hearts. In chapter 5, a small study is presented in which we retrospectively evaluated echocardiographic data on LV function from healthy children (control subjects) and from children with isolated AV block undergoing chronic epicardial pacing at either the RV or LV. After chronic LV pacing, LV diameter, geometry and function (fractional shortening) were at the level of healthy children, whereas chronic RV pacing was associated with a decrease in LV fractional shortening and a higher LV systolic eccentricity index.

Subsequently, we evaluated long-term influences of chronic ventricular pacing in children with isolated AV block in a large observational multi-center study, carried out at 27 centers. In this study, presented in chapter 6 , we retrospectively investigated the impact of ventricular pacing site on LV function in almost 300 children with permanent ventricular pacing for isolated AV block. The results indicated that the site of pacing is an important determinant of LV function, with LV fractional shortening being significantly higher in children with chronic LV pacing than in children with chronic RV pacing. Furthermore, LV fractional shortening was subnormal $(<28 \%)$ in $14 \%$ of the RV-paced children, whereas it was normal in all LV-paced children.

The retrospective nature of our gathered data, however, limited the evaluation to the measurement of LV shortening fraction. The purpose of the cross-sectional multi-center study presented in chapter 7 was therefore to evaluate the influence of different ventricular pacing 
sites on long-term LV function and synchrony in children with isolated AV block, using crosssectional assessment of multiple echocardiography parameters of LV function and dyssynchrony. Echocardiography data of 178 chronically paced children from 21 centers were systematically analyzed in a core lab. Left ventricular synchrony, pump function and contraction efficiency were significantly affected by pacing site and were best in children paced at the LV apex or lateral wall. Pacing from the RV free wall was related with decreased LV function, whereas LV apical and LV lateral wall pacing were associated with preserved LV function. In both the retrospective and cross-sectional evaluation, age at implantation, pre-implantation LV size and function, duration of pacing, DDD mode, QRS duration and presence of maternal auto-antibodies had no significant impact on long-term outcomes.

\section{Patients with a systemic right ventricle may benefit from single-site systemic ventricular pacing or biventricular pacing}

The study presented in chapter 8 concerns ventricular pacing in patients in whom the RV instead of the LV supports the systemic circulation. In these patients, having a high risk for dysfunction of the systemic ventricle, conventional non-systemic ventricular pacing (i.e. LV pacing in these patients) may even further increase the risk to develop RV dysfunction. In our study population of nine patients with a transposition of the great arteries treated by atrial redirection surgery (Senning or Mustard procedure), acute hemodynamic effects of endocardial systemic ventricular pacing and BiV pacing were significantly and equally better than those of nonsystemic ventricular pacing. As such, systemic ventricular pacing and BiV pacing may avoid additional pacing-induced risk for cardiac failure in patients with a systemic RV, and may therefore be the preferred strategies for chronic ventricular pacing in these patients.

In the last chapter of this thesis (chapter 9), the findings of above-mentioned studies are linked and put in broader perspective. Discussions are accompanied by recommendations for 'optimal pacing' and suggestions for related future research.

\section{Cardiac resynchronization therapy can be improved by better patient selection and more reliable optimization protocols}

The first part of the general discussion concerns optimization of CRT. On the one hand CRT might be reserved to a select subgroup of patients complying with current guidelines. On the other hand, since it may prevent or slow the progression to severe heart failure, the application of CRT might also be expanded to patients with only mild cardiomyopathy associated with dyssynchrony. However, complication rate and unnecessary costs are important issues in a mildly symptomatic patient cohort and make (upgrade to) BiV pacing in every patient with a conventional pacemaker indication or an intrinsic LBBB unfavorable. Until now, neither the longterm effect of (pacing-induced) dyssynchrony, nor the response to CRT can be predicted for the 
individual patient. The application of BiV pacing may better be limited to patients presenting overt echocardiographic signs of remodeling or deterioration of LV function. This recommendation is supported by the observation that adverse remodelling may be reversed by BiV pacing.

The method of optimization and its accuracy seem to be of critical importance for the reliability of the outcome of CRT-optimization. Variability of measurements used (the sum of biological and technical variability) is in the same range as the size of the effect to be measured. This implies that simply selecting the setting at which the highest single value is measured, is not the optimal approach to maximize the individual benefit of CRT. To decrease the impact of inevitable biological and measurement variability, multiple measurements should be taken and averaged for each device setting. Prospective, randomized studies, using adequate optimization protocols, are needed to clarify the real clinical impact of individual optimization on the patient's course.

\section{"Primum non nocere": optimal-site pacing may prevent pacing-induced cardiac dysfunction}

In the second part of the general discussion, several sites for chronic ventricular pacing are reviewed and clinical implications of these sites are discussed. Based on rationale and research, we suggest implantation of the lead for chronic ventricular pacing at the systemic ventricle, especially so when a surgical approach is also practically advised. Future studies with very longterm longitudinal follow-up and parameters other than those for systemic ventricular function are needed to investigate the hypothesis that systemic ventricular pacing indeed prevents pacing-induced heart failure. To facilitate the application of "optimal-site pacing" in clinical practice, it would be helpful when a range of functionally preferred pacing sites could be defined. It should therefore be investigated whether or not clinically relevant differences exist between pacing at different sites of the systemic ventricle. Future studies are also needed to investigate whether the difference between single-site systemic ventricular pacing and biventricular pacing is clinically relevant. 


\section{Samenvatting}

\section{Verbeteren van ventriculaire pacemakertherapie bij volwassenen en kinderen ter behandeling of voorkoming van hartfalen geïnduceerd door dyssynchronie}

De motieven voor bovengenoemde algemene intentie van het proefschrift en de daarin beschreven studies worden kort uiteengezet in hoofdstuk 1 en verder onderbouwd in hoofdstuk 2. De gemeenschappelijke achtergrond van het in dit proefschrift beschreven onderzoek betreft het feit dat een abnormaal elektrisch activatiepatroon van het hart kan samengaan met een slecht gecoördineerde, dyssynchrone samentrekking van het linker ventrikel (LV). Een abnormaal activatiepatroon kan niet alleen veroorzaakt worden door een gedeeltelijke onderbreking van het speciale elektrische geleidingssysteem, maar ook door kunstmatige elektrische stimulatie met behulp van een pacemaker. Zowel elektrisch stimuleren (i.e., pacen) van het rechter ventrikel (RV) als een onderbreking van het geleidingssysteem naar het LV, oftewel een linker bundeltakblok, zijn zowel geassocieerd met functionele achteruitgang en structureel remodelleren van het hart, alsook met een verhoogd risico op hartfalen.

Biventriculair (BiV) pacen, het kunstmatig stimuleren van beide ventrikels, kan de synchronie tussen beide ventrikels herstellen. Deze zogenoemde cardiale resynchronisatietherapie (CRT) is effectief gebleken in patiënten met ernstige dysfunctie van het LV geassocieerd met intrinsieke dyssynchronie of met door pacen geïnduceerde dyssynchronie. De individuele respons op CRT varieert echter in grote mate, hetgeen blijkt uit het feit dat 30-50\% van de patiënten die CRT krijgen een inadequate respons hebben. Om het behandelingseffect te vergroten zou CRT in verschillende opzichten verbeterd kunnen worden. Ons onderzoek in relatie tot de optimalisatie van CRT, met het streven dyssynchronie-geïnduceerd hartfalen in volwassenen te behandelen, wordt beschreven in de hoofdstukken 3 en 4 .

Bij patiënten met een aangeboren of verworven blokkade van de elektrische geleiding tussen atria en ventrikels, een atrioventriculair (AV) blok, is ventriculair pacen geïndiceerd indien het spontane ventriculaire ritme te laag is. De bewustwording van de potentieel nadelige effecten van conventionele RV-stimulatie heeft een zoektocht geïnitieerd naar alternatieve methoden voor chronisch ventriculair pacen. In de hoofdstukken 5-8 worden de studies gepresenteerd die betrekking hebben op het streven naar preventie van de functionele en structurele verslechtering van het hart die geassocieerd is met chronisch pacen in jonge patiënten.

\section{De overstap naar biventriculair pacen zou overwogen kunnen worden bij chronisch gestimuleerde patiënten met relatief milde cardiomyopatie.}

Cardiale resynchronisatietherapie probeert de negatieve effecten die het gevolg zijn van dyssynchronie van het LV ongedaan te maken door het bewerkstelligen van een beter gecoördineerd contractiepatroon. Het lijkt daarom aannemelijk dat alle patiënten met 
dyssynchronie van het LV baat zouden kunnen hebben bij CRT, ongeacht de mate van hartfalen of de oorsprong van de dyssynchronie. In de studie die wordt gepresenteerd in hoofdstuk 3 hebben we de effecten van BiV-pacen en RV-pacen vergeleken in een groep chronisch RVgestimuleerde patiënten die vanwege hun relatief milde cardiomyopathie (LV ejectiefractie $36 \pm 10 \%$ ) buiten de gebruikelijke criteria voor CRT vielen. Biventriculaire stimulatie resulteerde in een betere LV functie, minder LV dilatatie en minder klinische symptomen dan RV-pacen. Bovendien leek de respons op BiV-pacen niet gerelateerd aan de ernst van de LV-dysfunctie, noch aan de mate van de structurele veranderingen van het LV. Bij chronisch RV-gestimuleerde patiënten met relatief milde cardiomyopathie zou de toepassing van BiV-pacen dus overwogen kunnen worden, waarbij CRT niet zozeer dient als therapie voor hartfalen maar meer gebruikt wordt ter preventie van hartfalen.

\section{Voor de optimalisatie van cardiale resynchronisatietherapie kan gebruik gemaakt worden van non-invasief gemeten contouren van de arteriële drukgolf}

Acute en lange-termijn effecten van CRT zouden verder verbeterd kunnen worden door het afstemmen van de pacemakerinstellingen op de individuele patiënt. Maar zowel de procedures om de instellingen te optimaliseren, als de parameters die daarbij gebruikt worden, zijn nog steeds onderwerp van discussie. Bij optimalisatieprocedures heeft een patiëntvriendelijke, noninvasieve meting de voorkeur. Nexfin CO-Trek ${ }^{\circledR}$ (BMEYE B.V., Amsterdam, Nederland) is een techniek die veranderingen in het slagvolume kan bepalen uit non-invasief gemeten contouren van de arteriële drukgolf in de vinger. In hoofdstuk 4 onderzochten we de precisie en de toepasbaarheid van deze techniek in de optimalisatie van het AV-interval, door het met de arteriële drukgolfmetingen bepaalde slagvolume op drie manieren te vergelijken met de snelheid-tijd integraal van de aorta bloedvolumestroom gemeten met echo Doppler; de meting van slag-tot-slag veranderingen in slagvolume, de bepaling van relatieve effecten van CRT bij verschillende AV-intervallen, en de vaststelling van een optimaal AV-interval. Nexfin CO-Trek ${ }^{\circledR}$ had een goede precisie op alle drie niveaus. Daarom, en omdat het een makkelijke en patiëntvriendelijke methode betreft om hemodynamische veranderingen te evalueren, vinden wij dat het gebruikmaken van non-invasief gemeten contouren van de arteriële drukgolf in de vinger veelbelovend is in de individuele optimalisatie van CRT.

\section{In kinderen met een geïsoleerd atrioventriculair blok resulteert chronisch links ventriculair pacen, in vergelijking met rechts ventriculair pacen, in een betere functie van het linker ventrikel}

Gezien de gunstige hemodynamische effecten van LV-pacen in de acute situatie die in eerdere studies van onze groep werden aangetoond, onderzochten we of ook chronisch pacen op het LV in kinderen met een structureel normaal hart beter is dan RV-pacen. Hoofdstuk 5 betreft een kleine studie waarin we retrospectief, met behulp van bestaande echocardiografische data, de 
LV-functie evalueerden in kinderen met een compleet AV-blok die chronisch op het LV of RV gestimuleerd werden. Ook beoordeelden we de LV-functie in een controlegroep met gezonde kinderen. In kinderen met chronische LV-stimulatie waren de LV-diameter, geometrie en functie op het niveau van gezonde kinderen, terwij chronische RV-stimulatie geassocieerd was met een verminderde verkortingsfractie van het LV en een hogere systolische wandspanning van het LV.

Vervolgens evalueerden we de effecten van chronisch ventriculair pacen in kinderen met een geïsoleerd AV-blok in een grote, in 27 centra uitgevoerde, observationele studie. In deze in hoofdstuk 6 beschreven multicenter studie werd het belang van de paceplaats op LV-functie retrospectief onderzocht in bijna 300 kinderen met chronisch ventriculair pacen voor een geïsoleerd AV-blok. De resultaten van deze studie lieten zien dat de plaats waar gestimuleerd wordt een belangrijke invloed heeft op LV-functie, met een significant betere functie in kinderen met chronisch LV-pacen dan in kinderen met chronisch RV-pacen. Verder bleek dat de verkortingsfractie van het LV in $14 \%$ van de kinderen met RV-pacen subnormaal (<28\%) was, terwijl deze goed was in alle kinderen met LV-pacen.

Door het retrospectieve karakter van de studie waren vooral echografische data voorhanden waarmee de verkortingsfractie van het LV bepaald kon worden. Derhalve werd de multicenter studie die wordt gepresenteerd in hoofdstuk 7, zo opgezet dat middels een crosssectionele echocardiografische evaluatie meerdere parameters van LV-functie en dyssynchronie bepaald konden worden. Daarmee onderzochten we in kinderen met een geïsoleerd AV-blok de lange-termijn invloed van de paceplaats op de functie, synchronie en effectiviteit in contractie van het LV. Echocardiografische data van 178 chronisch gestimuleerde kinderen van 21 centra werden systematisch geanalyseerd in één laboratorium. Synchronie, pompfunctie en effectiviteit in contractie van het LV werden significant beïnvloed door paceplaats en waren het best in kinderen die werden gestimuleerd op de apex of laterale wand van het LV. Pacen van de vrije wand van het RV was sterk geassocieerd met een verminderde functie, terwijl pacen van de apex en vrije wand van het LV geassocieerd was met intacte LV-functie. Zowel in de retrospectieve als in de cross-sectionele evaluatie hadden leeftijd bij implantatie, grootte en functie van het LV vóór implantatie, duur van pacen, manier van pacen (DDD-mode), QRS-duur en aanwezigheid van maternale auto-antistoffen, geen significante impact op lange termijn uitkomsten.

\section{Patiënten met een systemische rechter ventrikel zouden profijt kunnen hebben van systemisch ventriculair of biventriculair pacen}

De studie die in hoofdstuk 8 wordt gepresenteerd, heeft betrekking op pacen in patiënten met een ondersteuning van de systemische circulatie door het RV, in plaats van het LV. Bij deze patiënten, die sowieso een verhoogd risico hebben op dysfunctie van het systemische RV, kan LVpacen (i.e., non-systemisch ventriculair pacen) het risico op RV-dysfunctie verder vergroten. In onze studiepopulatie van negen patiënten met een transpositie van de grote vaten behandeld met atriale redirectionele chirurgie (procedure volgens Senning of Mustard), waren de acute 
hemodynamische effecten van endocardiaal systemisch ventriculair en BiV-pacen significant beter dan die van endocardiaal non-systemisch ventriculair pacen. Daarom zou in patiënten met een systemische RV het additionele risico voor hartfalen dat wordt geïnduceerd door pacen, mogelijk voorkomen kunnen worden door systemisch ventriculair of BiV te pacen.

In het laatste hoofdstuk van dit proefschrift (hoodstuk 9) worden de bevindingen van bovengenoemde studies met elkaar verbonden en in een breder perspectief geplaatst. Discussies worden vergezeld door aanbevelingen voor de beste strategie voor pacen en door suggesties voor gerelateerd toekomstig onderzoek.

\section{Cardiale resynchronisatietherapie kan verbeterd worden door betere patiëntselectie en door betrouwbaardere optimalisatieprotocollen}

Het eerste deel van de algemene discussie behandelt de optimalisatie van CRT. Aan de ene kant zou CRT gereserveerd dienen te blijven voor een selecte subgroep van patiënten die voldoen aan de richtlijnen. Maar aangezien CRT de progressie naar ernstig hartfalen zou kunnen voorkomen of vertragen, zou aan de andere kant de toepassing van CRT misschien uitgebreid moeten worden naar patiënten met slechts milde dyssynchronie-geassocieerde cardiomyopathie. In een mild symptomatische patiëntengroep zijn het risico op complicaties en onnodige kosten echter belangrijke aspecten die het niet wenselijk maken om BiV-pacen toe te passen in iedere individuele patiënt met een indicatie voor conventioneel pacen of een intrinsiek linker bundeltakblok. Tot nu toe zijn noch de lange termijn effecten van dyssynchronie, noch de respons op CRT voor de individuele patiënt te voorspellen. Daarom, en omdat de negatieve effecten van dyssynchronie (deels) omkeerbaar zijn, zou de toepassing van BiV-pacen vooralsnog het beste gereserveerd kunnen worden voor patiënten die zich presenteren met duidelijke tekenen van remodelleren of dysfunctie van het LV.

Voor de betrouwbaarheid van de uitkomst van de optimalisatie van CRT lijken de optimalisatiemethode en zijn precisie van kritisch belang. De variabiliteit in de gebruikte metingen (de som van biologische en technische variabiliteit) is in dezelfde orde van grootte als het effect dat gemeten moet worden. Dit houdt in dat het eenvoudig selecteren van de instelling waarbij de beste waarde gemeten wordt niet de optimale aanpak is om het individuele voordeel van CRT te maximaliseren. Om het effect van onvermijdbare biologische variabiliteit en meetfouten te verminderen zouden voor iedere instelling meerdere metingen moeten worden gedaan en worden gemiddeld. Prospectieve gerandomiseerde studies die gebruik maken van adequate optimalisatieprotocollen die aan bovengenoemde voorwaarde voldoen, zijn nodig om de daadwerkelijke klinische impact van CRT-optimalisatie te bepalen. 


\section{"Primum non nocere"; pacen op de optimale paceplaats zou pacen- geïnduceerd hartfalen kunnen voorkomen}

In het tweede deel van de algemene discussie worden verschillende locaties voor chronisch ventriculair pacen besproken en worden de klinische implicaties van deze locaties bediscussieerd. Uitgaand van logica en de uitkomsten van wetenschappelijk onderzoek, adviseren wij om voor de toepassing van chronisch ventriculaire stimulatie de pacemakerdraad te implanteren op de systemische ventrikel. Dit advies geldt vooral indien een chirurgische benadering ook om praktische redenen wordt geadviseerd. Om de veronderstelling te onderzoeken dat stimulatie van het systemische ventrikel pacen-geïnduceerd hartfalen kan voorkomen, zijn vervolg studies nodig met langere follow-up en andere parameters dan alleen die voor de functie van de systemische ventrikel. Om de klinische toepasbaarheid van pacen op de optimale paceplaats te vergroten, zou het van voordeel zijn als een groter gebied van functioneel te prefereren paceplaatsen gedefinieerd zou kunnen worden. Het zou daarom onderzocht moeten worden of er klinisch-relevante verschillen bestaan tussen stimulatie van verschillende paceplaatsen op het systemische ventrikel. Ook zijn er toekomstige studies nodig om te onderzoeken of het verschil tussen biventriculair pacen en pacen op een enkele paceplaats op het systemische ventrikel klinisch relevant is. 



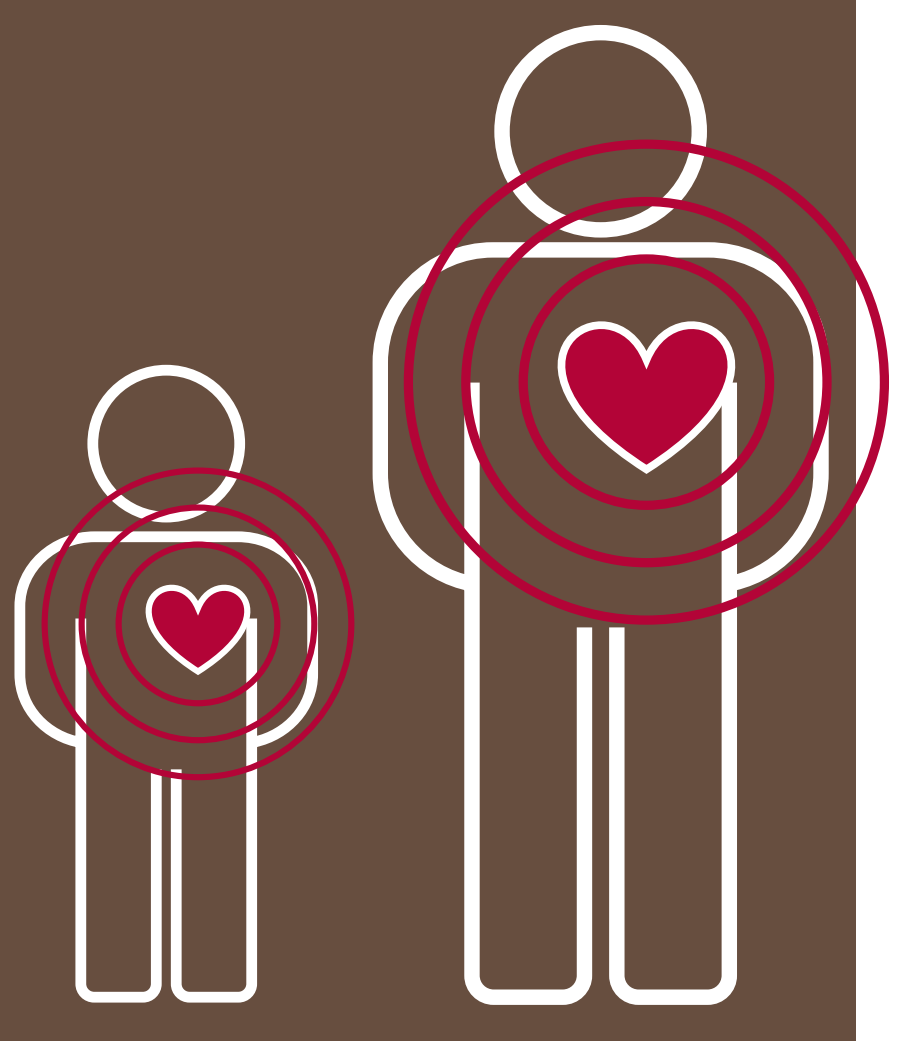

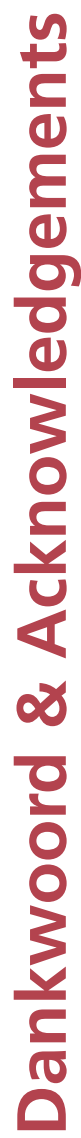


Dit proefschrift is tot stand gekomen mede door de inspanningen en kennis, en bovendien het enthousiasme, van vele anderen. Ik ben iedereen die in welke vorm dan ook heeft bijgedragen aan dit werk, heel erg dankbaar.

Graag wil ik allereerst nadrukkelijke dank uit laten gaan naar die mensen zonder wie dit proefschrift niet had kunnen bestaan.

Mijn promotores prof.dr. Tammo Delhaas en prof.dr. Frits W. Prinzen wil ik graag heel hartelijk bedanken voor hun onvoorwaardelijke en onuitputtelijke inzet om me te begeleiden in mijn werk en me op te leiden tot een kritisch, nauwkeurig en veelzijdig onderzoeker. Beste Tammo en Frits, ik zie het als groot geluk dat ik in de afronding van de studie Geneeskunde bij jullie terecht ben gekomen voor de invulling van mijn wetenschappelijke stage. Naast mijn eigen interesse in de kindercardiologie, zijn vooral jullie enthousiasme en de ongedwongen sfeer die uitging van besprekingen met jullie, motiverend geweest om de stage te vervolgen met een promotietraject. In dit traject hebben jullie het beste begeleiders-duo gevormd dat ik me had kunnen wensen!

Mijn derde promotor, prof.dr. Jan Janousek (University Hospital Motol, Prague) ben ik zeer erkentelijk voor een prettige en inspirerende samenwerking met betrekking tot een zeer aanzienlijk deel van dit proefschrift. Dear Jan, I thank you for our successful collaboration, which has been very inspiring to me. I look forward to working together in the future. I'm very honoured that you are one of my promotores.

Prof.dr. U. Schotten, prof.dr.ir. T. Arts, prof.dr. N. Blom, prof.dr. H.P. Brunner-La Rocca en prof.dr. L.J.I. Zimmermann, leden van de beoordelingscommissie, wil ik graag hartelijk bedanken voor de kritische beoordeling van mijn proefschrift.

Prof.dr. H.J.G.M. Crijns, is steeds zeer betrokken geweest bij de totstandkoming van mijn proefschrift. Beste prof. Crijns, bedankt voor het in mij en mijn promotores gestelde vertrouwen, de praktische steun, de waardevolle feedback op de manuscripten van onze studies, en de getoonde interesse.

Dr. W.Y.R. Vanagt was vanaf mijn eerste schreden in de wetenschappelijke wereld een voorbeeld en coach. Beste Ward, bedankt voor alle uitleg, praktische hulp en emotionele steun die je me de afgelopen jaren in mijn promotietraject hebt geboden. Dat ons beider werk "bekroond" werd met een 'Dr. E. Dekker beurs' van de Nederlandse Hartstichting was het summum. Ik hoop dat we de komende jaren nog veel zullen samenwerken en dat ik daarbij nog veel van je mag leren! 
Prof.dr. U. Bauersfeldt en dr. M. Tomaske (University Children's Hospital, Zürich), ben ik veel dank verschuldigd voor hun gastvrijheid in Zürich tijdens het eerste deel van mijn wetenschappelijke stage en voor de verdere samenwerking die resulteerde in mijn eerste wetenschappelijke publicatie (hoofdstuk 5). Dear Maren, I'm thankful for your hospitality and your collaboration in my first scientific project, as well as for your contribution to our multicenter studies on ventricular pacing in children.

Dr. K. Vernooy en dr. B. Dijkman wil ik bedanken voor het bieden van een lopend project (de "rijdende BiV-RV trein", hoofdstuk 3) waar ik in mocht stappen. Daarmee kon ik reeds aan het begin van mijn promotietraject aan de slag met de analyses van eerder verzamelde data.

Prof.dr. A. Auricchio (Fondazione Cardiocentro Ticino, Lugano), dr.ir. J. Settels (BMEYE B.V., Amsterdam), dr. R. Cornelussen en dr. B. Gerritse (Medtronic Bakken Research Center, Maastricht) en drs. B. Broers, wil ik bedanken voor de enorme inspanningen voor onze zogenaamde 'Nexfin-studie' (hoofdstuk 4). De intensieve brainstormsessies en discussies die gepaard gingen met deze studie, zijn steeds weer inspirerend en leerzaam geweest. Caro Angelo, I would like to thank you and your colleagues for the collaboration on the Nexfinproject. Moreover, our meetings at several international conferences were inspiring to me. I'm honoured with your participation in the corona. Beste Jos, bedankt voor je praktische en wetenschappelijke input. Beste Richard, bedankt voor je betrokkenheid en het in contact brengen met de juiste mensen. Beste Bart, bedankt voor je statistische adviezen. Beste Bernard, zowel het enthousiasme waarmee jij patiënten wist te motiveren mee te doen, als je eindeloze echografische inspanningen voor deze studie verdienen hier een eervolle vermelding.

Dr. D. Francis en dr. Z.I. Whinnett (St. Mary's Hospital and Imperial College, London) ben ik dankbaar voor de tips-'n-tricks met betrekking tot CRT-optimalisatie. Dear Darrel, I would like to thank you for the inspiring (email)-discussions on "how (not) to optimize CRT". Dear Zach, 'un grand merci' for your help and enthusiasm in Bordeaux.

Dr. J.B. Thambo, dr. P. Bordachar, prof.dr. M. Haïssaguerre, drs. Ph. Ritter, drs. X. Iriart, drs. M. de Guillebon et collègues, de I'Hôpital Cardiologique du Haut-Lévêque, (PessacBordeaux) ben ik zeer dankbaar voor hun gastvrijheid en hun enthousiasme om een jaar lang samen te werken aan het project waarvoor ik de beurs van de Nederlandse Hartstichting ontving. Je vous remercie de m'avoir accordée votre confiance en m'offrant l'opportunité de faire mes travaux de recherche chez vous. Merci de votre soutien. J'ai eu un grand plaisir à travailler à vos côtés et de profiter de votre savoir. 
Alle coauteurs van de multi-center studies (hoofdstukken 6 en 7) ben ik zeer erkentelijk voor hun bijdragen. We could never have gathered so many data without the enthusiastic (financially unrewarded) participation of so many colleagues from all over the world. Their efforts for collecting data as well as their constructive feedback to the manuscripts are very much appreciated.

Alle patiënten die hebben geparticipeerd in de studies beschreven in dit proefschrift ben ik veel dank verschuldigd.

De Nederlandse Hartstichting ben ik zeer erkentelijk voor de 'Dr. E. Dekker beurs' voor kindercardiologisch onderzoeker, die ik ontving voor de uitvoering van de studie naar de effecten van ventriculair pacen in jongvolwassenen met een systemische rechter ventrikel (hoofdstuk 8). Dit project is in veel opzichten verrijkend geweest voor mijn onderzoekstraject.

Zonder goede collegae, vrienden en familie, zou het voor mij niet mogelijk zijn geweest onderzoek te doen. Daarom wil ik hier graag diegenen noemen die op andere (in)directe wijze hebben bijgedragen aan de totstandkoming van dit proefschrift.

Alle collegae van de vakgroepen Fysiologie, Biomedische Technologie, en Kindergeneeskunde wil ik bedanken voor de getoonde interesse, de kritische inbreng en praktische hulp, en bovenal voor de bemoedigende woorden en het bieden van de mogelijkheid om frustraties te delen wanneer dat nodig was. Een speciaal woord van dank aan diegenen met wie ik de afgelopen jaren het meeste onderzoekslief en -leed heb gedeeld: Joost Lumens en Wilco Kroon. Beste Wilco en Joost, bedankt voor de grote portie humor die jullie me de afgelopen jaren hebben gebracht. Ik hoop dat WIJ (i.e., Wilco, Irene, Joost) tot in de verre toekomst nog vaak met elkaar zullen lachen! Marc Strik, mijn steun en toeverlaat voor de steeds complexer wordende excel-bestanden en mijn favoriete congresmaatje. Beste Marc, je bent in de afgelopen jaren een goede vriend geworden. Met jou als paranimf voel ik me gesterkt in alle opzichten! Daarnaast wil ik jou, en mijn andere kamergenootjes Marloes Gelissen, Ilka LorenzenSchmidt, Caroline van Deursen, Patrick Houthuizen, Sandra Eliautou en Sylvain Ploux bedanken voor de fijne samenwerking en het delen van vreugdes en frustraties.

Student Ben Hermans ben ik ook veel dank verschuldigd: zonder zijn MathLab hulp had ik de Nexfin studie (hoofdstuk 4) wellicht niet tot een goed einde weten te brengen. Onze goede en leuke samenwerking heeft ertoe bijgedragen dat het doen van onderzoek me ook in die periode (waarin 'de flow' iets minder voortvarend was) voldoening gaf. Ook de samenwerking met de studenten Michel Janssen, Loes de Vaan en Guusje Vugts heb ik als heel inspirerend ervaren! 
Zonder vrienden en familie zou ik ongelukkig zijn geweest. Ik ben jullie allen meer dan dankbaar voor de getoonde interesse in mijn onderzoek, maar vooral voor het doen vergeten van de onderzoeksperikelen en het samen genieten van het leven.

Lieve papa en mama, ik ben jullie enorm dankbaar voor het feit dat jullie me van jongs af aan hebben gestimuleerd om mijn talenten zo goed mogelijk te ontplooien en mijn best te doen om mijn 'non-talenten' te verbeteren. Het heeft me gevormd tot de persoon die ik nu ben, en het heeft bijgedragen aan alles wat ik tot nu toe heb bereikt. Bedankt voor alle kansen, de steun en de onvoorwaardelijke liefde die jullie me bieden.

Lieve Martijn en Marie-Sophie, broer en zusje, ik heb het getroffen met jullie! Een leven zonder jullie is niet voor te stellen. Lieve Martijn, ik heb groot respect voor jouw doorzettingsvermogen! Het betekent veel voor me dat je mijn paranimf wil zijn. Lieve MarieSophie, jij bent de enige in ons gezin die niet werkt in de gezondheidszorg, maar zich juist richt op de uiterlijke verzorging van de mens. Ik vind het mooi dat jij zo anders bent dan ik, omdat je juist daardoor mijn leven verrijkt! Ik ben trots op je.

Lieve Joost, het is onmogelijk om in woorden uit te drukken wat jij voor mij betekent. De bijdragen die jij hebt geleverd om mijn promotietraject en -boekje tot een goed einde te brengen, zijn talloos. Ik ben je daarvoor meer dan dankbaar. Bovenal ben ik blij dat mijn promotietraject er ook toe heeft geleid dat wij elkaar gevonden hebben. Jij hebt de gave om altijd, in welke gemoedstoestand ik ook ben, een lach op mijn gezicht te toveren. Mijn wereld is veel mooier en leuker met jou! 



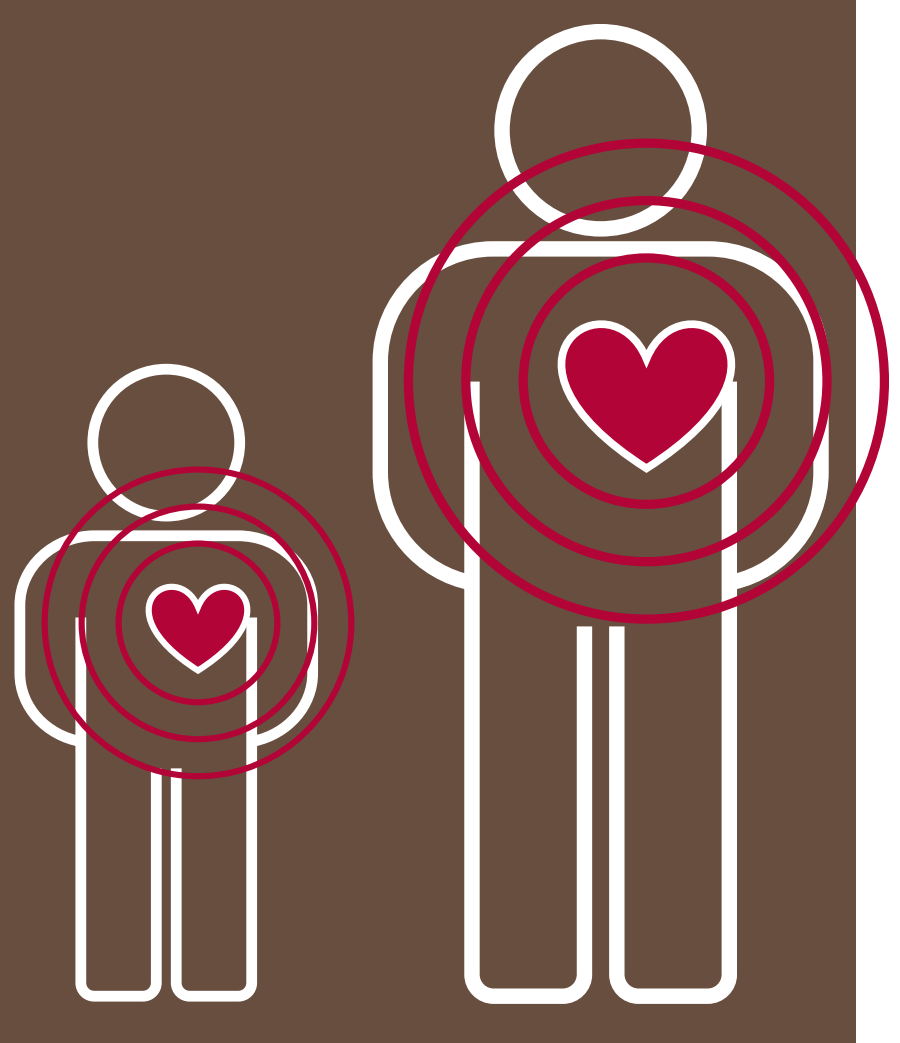

$\frac{1}{0}$

n

0

(1)

$+$

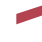

$\frac{E}{5}$ 


\section{Irene Elisabeth Gerardine van Geldorp}

was born on April 6, 1981 in Utrecht, The Netherlands.

From 1993 until 1999, she attended secondary education at "Stedelijk Gymnasium" in Breda where she obtained her Gymnasium diploma. In 1999, she started her medical training at the Faculty of Health and Life Sciences at Maastricht University. In parallel to her medical training, she attended courses of psychology and completed a facultative internship in pediatric cardiology at "Ospedale Bambin' Gesú" in Rome (Italy), from September 2003 until February 2004. She became 'doctorandus' in Medicine (equivalent of Master of Science) in 2004 and graduated as Medical Doctor in July 2006. Subsequently, she gained clinical experience in pediatrics at Orbis Medisch Centrum in Sittard and as of November 2006, she was a resident in pediatrics at Maastricht University Medical Center. From July 2007 until 2012, she worked as a PhD-student at Cardiovascular Research Institute Maastricht (Maastricht University) under supervision of prof.dr.

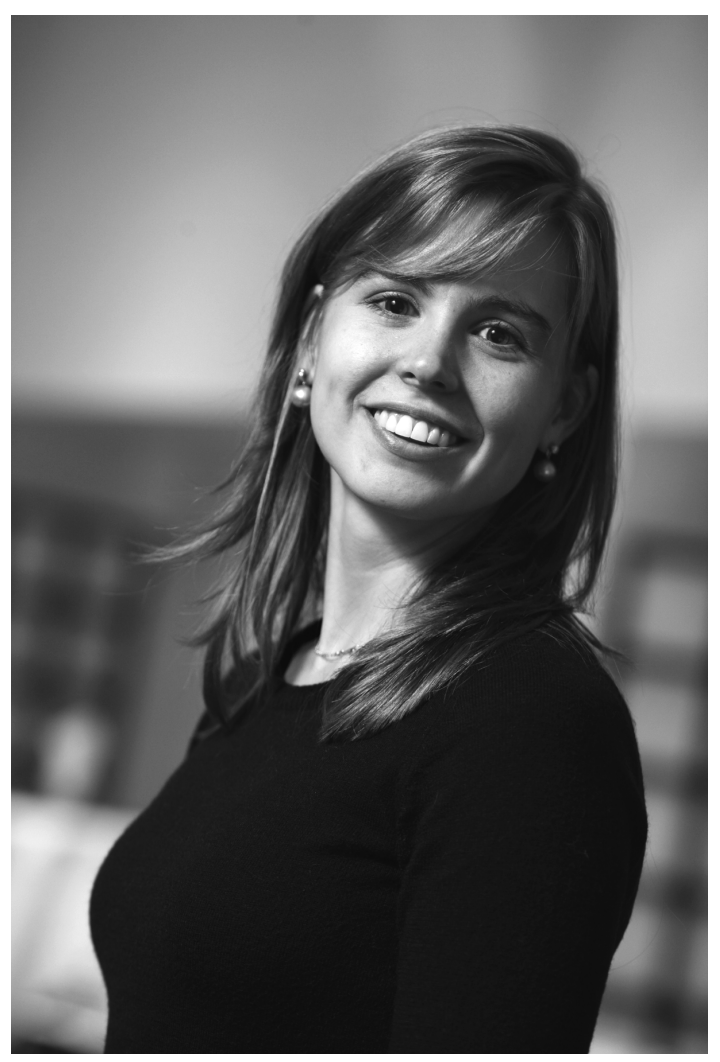

Tammo Delhaas and prof.dr. Frits Prinzen, and in close collaboration with prof.dr. Jan Janoušek from University Hospital Motol, Prague (Czech Republic). The aim of her PhD-project was to treat and to avoid dyssynchrony-induced cardiac failure by improving ventricular pacing therapy in adults and children. During this PhD-project, she worked in close collaboration with many other colleagues from all over the world. In 2010, she was rewarded with a grant of the Dutch Heart Foundation (Dr. E. Dekker grant for research fellow in pediatric cardiology) for a research project in collaboration with $\mathrm{dr}$. J.B. Thambo from "Hôpital Cardiologique du Haut-Lévêque", Bordeaux-Pessac (France). She worked in Bordeaux from January 2011 until February 2012. When she returned to Maastricht, she started working as a resident in pediatrics at Maastricht University Medical Center and from July 2012 she commenced her training in pediatrics. 


\section{List of Publications}

van Geldorp IE, Bordachar P, Lumens J, De Guillebon M, Whinnett ZI, Prinzen FW, Haissaguerre $M$, Delhaas T, Thambo JB. Acute hemodynamic benefits of biventricular and single-site systemic ventricular pacing in patients with a systemic right ventricle. Submitted 2012.

Janousek J, van Geldorp IE, Krupickova S, Rosenthal E, Tomaske M, Fruh A, Elders J, Hiippala A, Kerst G, Gebauer RA, Kubus P, Frias P, Gabbarini F, Clur SA, Nagel B, Ganame X, Papagiannis J, Marek J, Tisma-Dupanovic S, Tsao S, Nurnberg JH, Wren C, Friedberg MK, De Guillebon M, Prinzen FW, Delhaas T. Permanent Cardiac Pacing in Children - Choosing the Optimal Pacing-site: A MultiCenter Study. Revision under review for Circulation 2012.

van Geldorp IE, Vanagt WY, Vugts G, Willems R, Rega F, Gewillig M, Delhaas T. Late recovery of atrioventricular conduction after post-surgical chronic atrioventricular block is not exceptional. J Thorac Cardiovasc Surg 2012. Epub ahead of print.

Horovitz A, van Geldorp IE, Roubertie F, Thambo JB. Large Right Ventricular Fibroma in a 6Month-Old Infant. Pediatr Cardiol 2012. Epub ahead of print.

Horovitz A, De Guillebon M, van Geldorp IE, Bordachar P, Roubertie F, Iriart X, Douard H, Haissaguerre $M$, Thambo JB. Effects of non-systemic ventricular pacing in patients with transposition of the great arteries and atrial redirection. J Cardiovasc Electrophysiol 2012; 23(7):766-770.

van Geldorp IE, Delhaas T, Gebauer RA, Frias P, Tomaske M, Friedberg MK, Tisma-Dupanovic S, Elders J, Fruh A, Gabbarini F, Kubus P, Illikova V, Tsao S, Blank AC, Hiippala A, Sluysmans T, Karpawich P, Clur SA, Ganame X, Collins KK, Dann G, Thambo JB, Trigo C, Nagel B, Papagiannis J, Rackowitz A, Marek J, Nurnberg JH, Vanagt WY, Prinzen FW, Janousek J. Impact of the permanent ventricular pacing site on left ventricular function in children: a retrospective multicentre survey. Heart 2011; 97(24):2051-2055.

Pabari PA, Willson K, Stegemann B, van Geldorp IE, Kyriacou A, Moraldo M, Mayet J, Hughes AD, Francis DP. When is an optimization not an optimization? Evaluation of clinical implications of information content (signal-to-noise ratio) in optimization of cardiac resynchronization therapy, and how to measure and maximize it. Heart Fail Rev 2011; 16(3):277-290.

van Geldorp IE, Vanagt WY, Prinzen FW, Delhaas T. Chronic ventricular pacing in children: toward prevention of pacing-induced heart disease. Heart Fail Rev 2011; 16(3):305-314. 
van Geldorp IE, Delhaas T, Hermans B, Vernooy K, Broers B, Klimusina J, Regoli F, Faletra FF, Moccetti T, Gerritse B, Cornelussen R, Settels JJ, Crijns HJ, Auricchio A, Prinzen FW. Comparison of a non-invasive arterial pulse contour technique and echo Doppler aorta velocity-time integral on stroke volume changes in optimization of cardiac resynchronization therapy. Europace 2011; 13(1):87-95.

van Geldorp IE, Vernooy K, Delhaas T, Prins MH, Crijns HJ, Prinzen FW, Dijkman B. Beneficial effects of biventricular pacing in chronically right ventricular paced patients with mild cardiomyopathy. Europace 2010; 12(2):223-229.

van Deursen C, van Geldorp IE, Rademakers LM, van Hunnik A, Kuiper M, Klersy C, Auricchio A, Prinzen FW. Left ventricular endocardial pacing improves resynchronization therapy in canine left bundle-branch hearts. Circ Arrhythm Electrophysiol 2009; 2(5):580-587.

van Geldorp IE, Vanagt WY, Bauersfeld U, Tomaske M, Prinzen FW, Delhaas T. Chronic left ventricular pacing preserves left ventricular function in children. Pediatr Cardiol 2009; 30(2):125-132. 
"On ne voit bien qu'avec le cœur"

(Le Petit Prince, Antoine de Saint-Exupéry) 
